\title{
TECHNIQUES FOR ESTIMATING THE QUANTITY AND QUALITY OF STORM RUNOFF FROM URBAN WATERSHEDS OF JEFFERSON COUNTY, KENTUCKY
}

By Ronald D. Evaldi and Brian L. Moore

U.S. GEOLOGICAL SURVEY

Water-Resources Investigations Report 94-4023

Prepared in cooperation with the LOUISVILLE AND JEFFERSON COUNTY METROPOLITAN SEWER DISTRICT 


\section{U.S. DEPARTMENT OF THE INTERIOR \\ BRUCE BABBITT, Secretary}

U.S. GEOLOGICAL SURVEY

GORDON P. EATON, Director

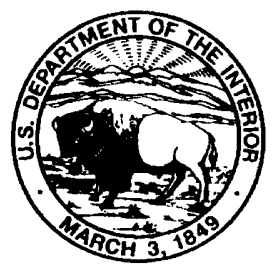

For additional information write to:

Copies of this report can be purchased from:

District Chief

U.S. Geological Survey

2301 Bradley Avenue

Louisville, KY 40217
U.S. Geological Survey, ESIC

Open-File Reports Section

Box 25286, MS 517

Denver Federal Center

Denver, CO 80225 


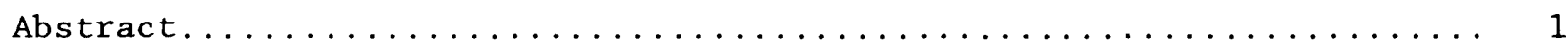

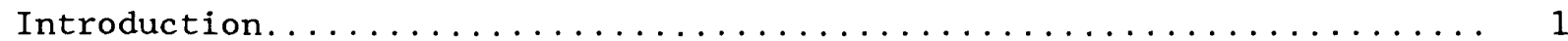

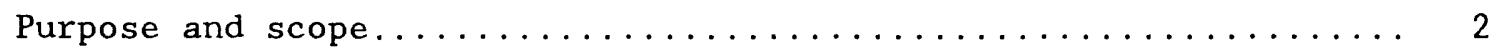

Description of study area........................ 2

Climate............................. 4

Population and land use.................... 4

Surface-water hydrology...................... 6

Stormwater data for Jefferson County.................... 6

Techniques for estimating the quantity and quality of storm runoff.... 13

Loads of constituents in storm runoff, and quantity of runoff..... 13

Mean concentrations of constituents in storm runoff........... 19

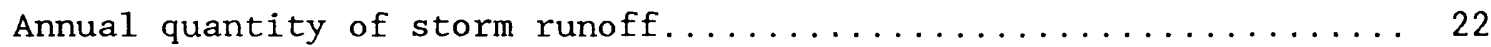

Annual loads of constituents in storm runoff.............. 25

Mean annual loads of constituents in storm runoff............ 29

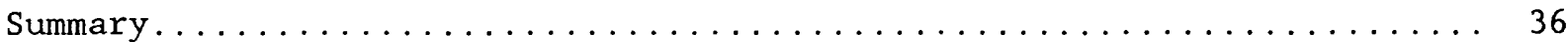

References cited............................ 38

Appendix: Supplemental streamflow and water-quality information...... 40

\section{ILLUSTRATIONS}

Figure

1-4. Maps showing:

1. Major stream basins in Jefferson County, Kentucky....... 3

2. Generalized land use in Jefferson County, Kentucky...... 5

3. Stormwater-outfall sampling sites

in Jefferson County, Kentucky............... 7

4. Stream-water-quality sampling sites

in Jefferson County, Kentucky............... 11

5. Graphs showing partitioned flow of selected streams

in Jefferson County, Kentucky, $1988-92 \ldots \ldots \ldots \ldots \ldots \ldots$

TABLES

Table 1. Stormwater-outfall sampling sites in Jefferson County, Kentucky, and primary land uses in their watersheds........

2. Rainfall and runoff associated with stormwater samples collected in Jefferson County, Kentucky, January 1991-

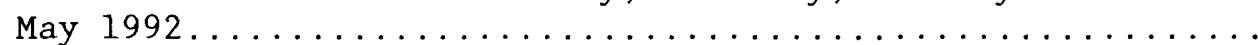

3. Loads and event mean concentrations of selected constituents in storm runoff measured at stormwater outfalls in Jefferson County, Kentucky, January 1991-May 1992.........

4. Stream-water-quality sampling sites in Jefferson County, Kentucky, and potential sources of contaminants in their watersheds. 
5. Land uses and percentage of impervious cover within the watersheds of selected stream-water-quality and stormwateroutfall sampling sites in Jefferson County, Kentucky........

6. Adjustments to regional regression models for estimating loads of selected constituents in storm runoff and quantity of storm runoff in single storms from urban watersheds of Jefferson County, Kentucky....................... 15

7. Adjustments to three-variable regional regression models for estimating loads of selected constituents in storm runoff in single storms from urban watersheds of

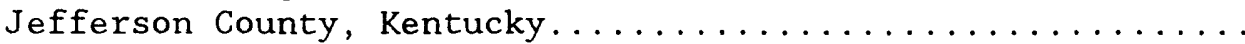

8. Summary of statistics for regression models of mean constituent concentrations in storm runoff in single storms from urban watersheds of Jefferson County,

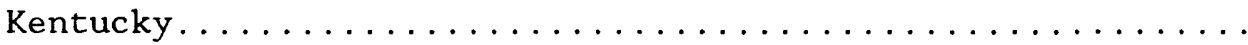

9. Summary of statistics for regression models of annual loads of selected constituents in storm runoff from urban watersheds of Jefferson County, Kentucky...............

10. Adjustments to regional regression models for estimating mean annual loads of selected constituents in storm runoff from urban watersheds of Jefferson County, Kentucky........

11. Estimates of mean annual loads of selected constituents in storm runoff from urban watersheds of Jefferson County,

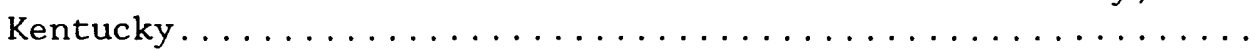

12. Average annual total streamflow and average annual base flow in streams in Jefferson County, Kentucky, 1988-92.....

13. Statistical summary of constituent concentrations in streams in Jefferson County, Kentucky, 1988-92..........

14. Estimates of mean annual constituent loads in runoff from urban watersheds of Jefferson County, Kentucky, 1988-92....

15. Estimates of mean annual loads of selected constituents in base flow in urban watersheds of Jefferson County,

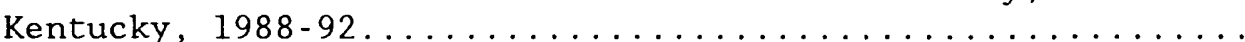


Multiply

inch (in.)

mile (mi)

pound (1b)

ton (ton)

acre

square mile $\left(\mathrm{mi}^{2}\right)$

cubic foot $\left(f t^{3}\right)$

cubic foot per second $\left(\mathrm{ft}^{3} / \mathrm{s}\right)$
By

25.4

1.609

0.4536

907.2

0.4047

2.590

28.32

28.32
To obtain

millimeter

kilometer

kilogram

kilogram

square hectometers

square kilometer

cubic decimeter

cubic decimeter per second

Temperature in degrees Fahrenheit can be converted to degrees Celsius as follows:

$$
{ }^{\circ} \mathrm{C}=\left({ }^{\circ} \mathrm{F}-32\right) / 1.8
$$

Abbreviated water-quality units used in this report: Chemical concentrations and water temperatures are given in metric units. Chemical concentration is given in milligrams per liter $(\mathrm{mg} / \mathrm{L})$ or micrograms per liter $(\mu \mathrm{g} / \mathrm{L})$.

Milligrams per liter is a unit expressing the concentration of chemical constituents in solution as weight (milligrams) of solute per unit volume (liter) of water. One thousand micrograms per liter is equivalent to one milligram per liter. For concentrations less than 7,000 mg/L, the numerical value is the same as for concentrations in parts per million. 


\section{TECHNIQUES FOR ESTIMATING THE QUANTITY AND QUALITY OF STORM RUNOFF FROM URBAN WATERSHEDS OF JEFFERSON COUNTY, KENTUCKY}

By Ronald D. Evaldi and Brian L. Moore

\section{ABSTRACT}

This report presents techniques for estimation of storm-runoff volumes, and mean concentrations and loads of selected constituents in storm runoff from urban watersheds of Jefferson County, Ky. Estimation models were developed on the basis of runoff volumes, and concentrations and loads of selected constituents in runoff measured at 6 stormwater outfalls and 25 streams in Jefferson County. In addition, previously developed regional estimation models were evaluated to assess their suitability for use in the county. Adjustments to the previously developed models were determined from comparisons to data obtained in Jefferson County.

The estimation techniques consist of sets of linear regression models for estimating mean concentrations and total loads of selected constituents in single storms, the quantity of the storm runoff, and annual and mean annual loads of selected constituents in storm runoff. Constituents modeled include dissolved oxygen, biochemical and chemical oxygen demand, dissolved and suspended solids, volatile residue, nitrogen, phosphorus and phosphate, calcium, magnesium, barium, copper, iron, lead, and zinc. Model estimations are a function of drainage area, percentage of imperviousness, climatological data, and land use.

\section{INTRODUCTION}

Jefferson County, Ky., which includes the city of Louisville, is a rapidly developing urban area. As urbanization has progressed, rural land has been replaced by residences, businesses, industrial facilities, shopping centers, and parking lots. Associated with urbanization is a change in the types and quantities of contaminants discharged to the surface waters of the county. Storm runoff can wash contaminants, which accumulate during dry periods, into urban drainage systems and degrade the surface-water quality of many streams and drainage channels. Many contaminants in urban runoff are primarily by-products of human activity and include organic debris, sediments, nutrients, petroleum-based products, and potentially toxic chemicals, such as heavy metals and pesticides.

Urban planners and managers need information on the quantity and quality of runoff to plan adequately for the effects of storm runoff from urban areas. Quantity of runoff is commonly expressed as volumes; quality of runoff is commonly expressed in terms of constituent concentrations or loads. (The load of a constituent in a stream is the concentration of that constituent multiplied by the discharge.) The constituents that contribute to the total stream load are often from point and nonpoint sources. Nonpoint-source contaminants generally are transported to receiving waters in runoff resulting 
from storms ${ }^{1}$. Estimates of loads of selected constituents in storm runoff provides a measure of nonpoint-source effects on water quality. Estimates of annual loads and event mean concentrations of selected constituents are required as part of the National Pollutant Discharge Elimination System permit for discharges from municipal separate storm-sewer systems (U.S. Environmental Protection Agency, 1992). (The event mean concentration of a constituent is the mean of that constituent in runoff during a storm from the rise, through the crest, together with the recession that follows the crest.)

In 1991, the Louisville and Jefferson County Metropolitan Sewer District (MSD) and the U.S. Geological Survey (USGS) began a cooperative program to assess the quality of stormwater runoff in the county. The program involved collection of a number of samples for determination of stormwater quality. Quantification of constituent concentrations in the stormwater and estimation of constituent transport from the watersheds can help to describe the effects of urbanization on water quality.

\section{Purpose and Scope}

This report describes techniques for estimating the quantity and quality of storm runoff from urban watersheds of Jefferson County, Ky. Previously developed regional estimation models were evaluated to assess their suitability for use in the county. These models were adjusted, and additional new models were developed on the basis of storm data measured in Jefferson County. Regression models are presented for estimation of (1) selected constituent concentrations in storm runoff, (2) runoff volumes and selected constituent loads in runoff from single storms, and (3) annual total volumes of runoff and loads of selected constituents in runoff. The main constituents of interest for this study include chemical oxygen demand, biochemical oxygen demand, dissolved solids, suspended solids, total nitrogen, total $\mathrm{kjeldahl}$ nitrogen, total phosphorus, dissolved phosphorus, total cadmium, total copper, total lead, and total zinc.

\section{Description of Study Area}

Jefferson County covers $386 \mathrm{mi}^{2}$ of the north-central part of Kentucky along the Ohio River (fig. 1) (Louisville Chamber of Commerce, 1992). Within its borders is Louisville, the largest city and the most densely populated area of the State.

${ }^{1}$ Approximately 69 storms, defined as 0.1 in. accumulation with at least 0.01 in. each hour, occur each year in Jefferson County (Steurer and Nold, 1986). 


\section{EXPLANATION}

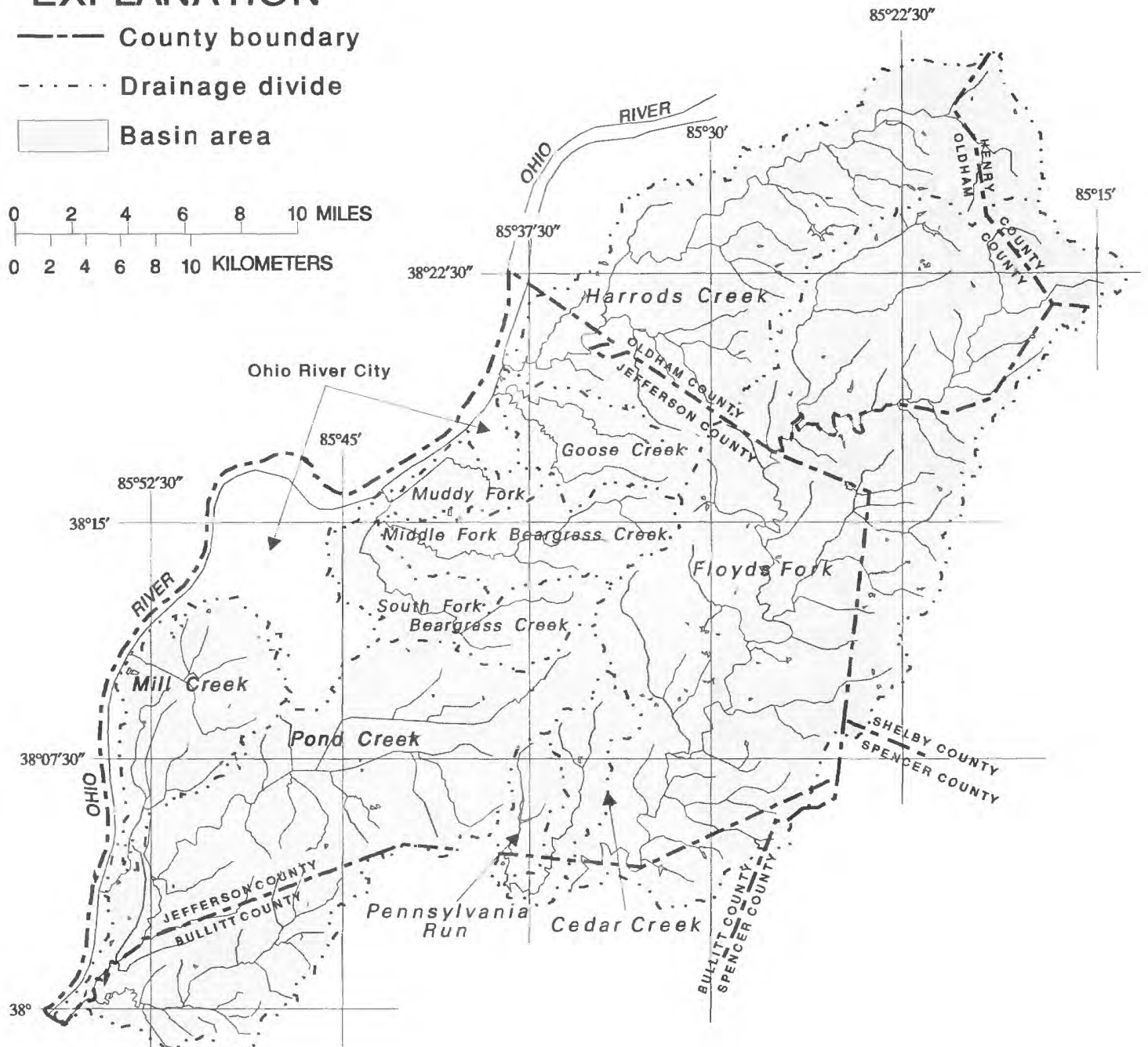

INDEX MAP

Base from U.S. Geologlcal Survey, digltal data, 1:100,000, 1983 Unlversal Transverse Mercator projection, Zone 16

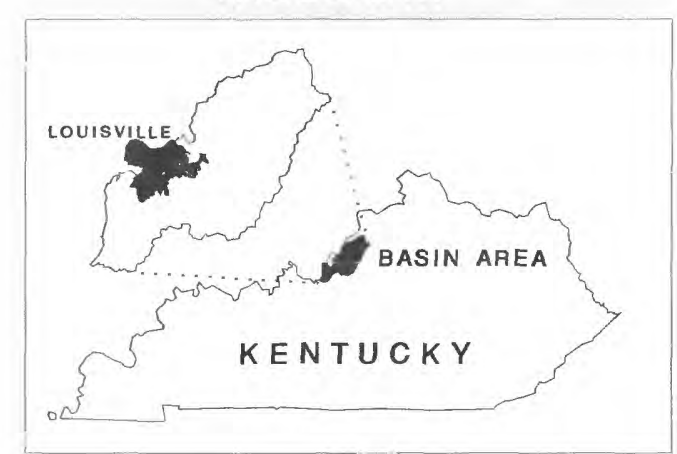

Figure 1.--Major stream basins in Jefferson County, Kentucky. 


\section{Climate}

The climate of Louisville, Ky., is classified as "moist-continental" by Strahler and Strahler (1979). It is characterized by changeable weather and only short periods of extreme conditions. Weather systems generally track either north from the Gulf of Mexico, bringing warm, moist air in the summer, or southeast from Canada, bringing occasional arctic air masses to the area in the winter. As a result, winters are moderately cold (temperatures rarely below $0^{\circ} \mathrm{F}$ ), and summers are warm (temperatures rarely above $100^{\circ} \mathrm{F}$ ). The coldest month is January, during which the daily minimum temperature averages $26.2^{\circ} \mathrm{F}$; the warmest month is July, during which the daily maximum temperature averages $88.1^{\circ} \mathrm{F}$ (National Oceanic and Atmospheric Administration, 1990).

Based on records for 1960-90, the average annual precipitation at Louisville is 43.0 in. (National Oceanic and Atmospheric Administration, 1990). Generally, October is the driest month and March is the wettest. Thunderstorms usually contribute substantially to the rainfall in the spring and summer. Snow usually occurs from November through March, although it has fallen as late as April and as early as October. Average annual snowfall is 16.6 in. (5.4 in. in January).

\section{Population and Land Use}

According to the 1990 U.S. Census, the population of Jefferson County is approximately 665,000 (Louisville Chamber of Commerce, 1992). This figure represents a 3-percent decline from the 1980 census and a 4-percent decline since 1970. The Louisville Chamber of Commerce, however, projects that the population of Jefferson County will grow to 673,000 by the end of 1994 .

Generalized land-use regions of Jefferson County are shown in figure 2. The sources of the land-use data shown in figure 2 are 1983 National Atmospheric and Space Administration high-altitude aerial photographs and National High-Altitude Photography program photographs digitized at a scale of 1:250,000 (U.S. Geological Survey, 1986). The degree of change in land use since 1983 is unknown; however, on the basis of this 1983 land-use information, most commercial and industrial land is within the Louisville city limits. In this report, agricultural and forest and wetland land uses were considered as nonurban.

Within and immediately surrounding the city limits, residential land use predominates (with some exceptions). The least populated and least developed watersheds of Jefferson County are Pennsylvania Run, which has one population center, and Cedar Creek, which has three population centers (fig. 1). Most agricultural and forest land is in the eastern, southern, and southwestern parts of the county. Industrial areas are also in these parts of the county, however, and include parts of an industrial park in the Floyds Fork watershed, an industrial park and truck-assembly plant in the Harrods Creek watershed, and large manufacturing facilities in the Pond Creek watershed. 


\section{EXPLANATION LAND USE}

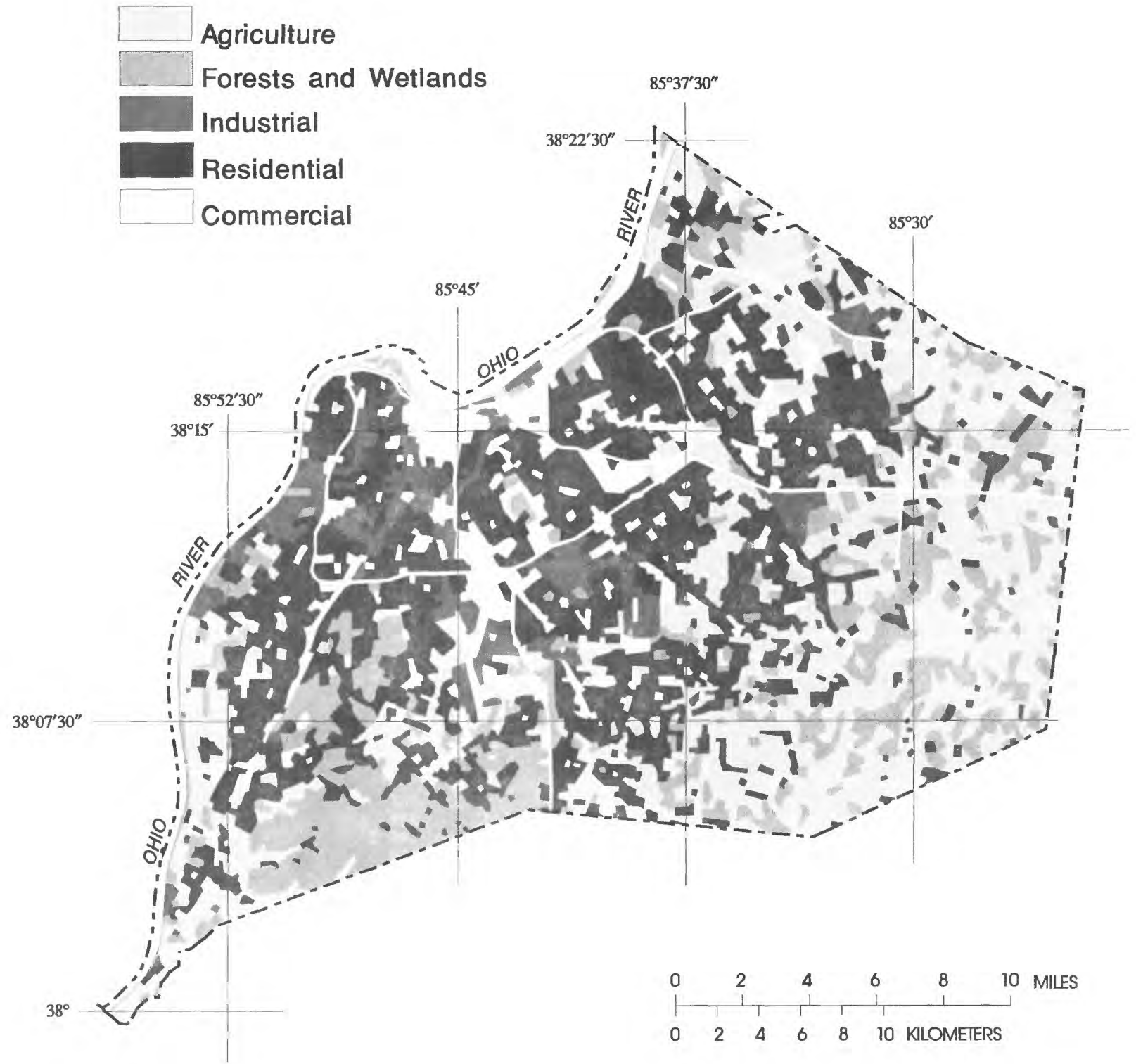

Base from U.S. Geologlcal Survey, dlgltal data, 1:100,000, 1983 Land use from digital data, 1:250,000,1983

Universal Transverse Mercator projection, Zone 16

Figure 2.--Generalized land use in Jefferson County, Kentucky. 


\section{Surface-Water Hydrology}

Ten stream systems course through Jefferson County and also drain parts of five surrounding counties (fig. 1). These stream systems are Harrods Creek, Goose Creek, Muddy Fork, Middle Fork Beargrass Creek, South Fork Beargrass Creek, Floyds Fork, Cedar Creek, Pennsylvania Run, Pond Creek, and Mil1 Creek. Their watersheds range in size from $8.5 \mathrm{mi}^{2}$ (Pennsylvania Run) to $222 \mathrm{mi}^{2}$ (Floyds Fork) and have a combined area of approximately $600 \mathrm{mi}^{2}$.

The Louisville metropolitan area and other parts of the county that drain directly to the Ohio River are known locally as the Ohio River City area (fig. 1). The Louisville metropolitan area consists of a dense commercial central business district that is drained mainly by a complex system of combined sanitary and storm sewers, with few open channels.

\section{STORMWATER DATA FOR JEFFERSON COUNTY}

Stormwater samples were collected in Jefferson County from storm outfalls in six small watersheds identified as having a specific predominant land use. These samples were collected to describe the chemical characteristics of stormwater in Jefferson County. Total storm-runoff volume was measured for most sampled storms and used in conjunction with the sample analyses to compute loads of selected constituents in the storm runoff. These data were supplemented with constituent-load computations from 25 stream-water-quality sampling sites in the county.

The stormwater outfalls used for sampling (fig. 3) and the primary land uses in their watersheds are listed in table 1.

Table 1. Stormwater-outfall sampling sites in Jefferson County, Kentucky, and primary land uses in their watersheds

Site number and name

SR1 Tributary to South Fork Beargrass Creek

SR2 Tributary to Hite Creek

SR3 Tributary to Middle Fork Beargrass Creek

SR4 Tributary to Northern Ditch

SR5 Tributary to Big Run

SR6 Tributary to Middle Fork Beargrass Creek
Primary land use in watershed

high-density residential

light industrial

low-density residential

heavy industrial

commercial

commercial

At least three storms that occurred at least 1 month apart were sampled at each of the six stormwater outfalls from January 1991 through May 1992 (table 2). Stormwater sampling generally followed a dry-weather period of at least 72 hours. The sample data used in this report represented the entire flow period of the stormwater outfall for each storm. Event mean concentrations and loads measured at the six storm-outfall sites in Jefferson 


\section{EXPLANATION}

\section{Stormwater-outfall basin}

SR6 Stormwater-outfall sampling site and identifier (see table 1 for site description)

$85^{\circ} 37^{\prime} 30^{\prime \prime}$

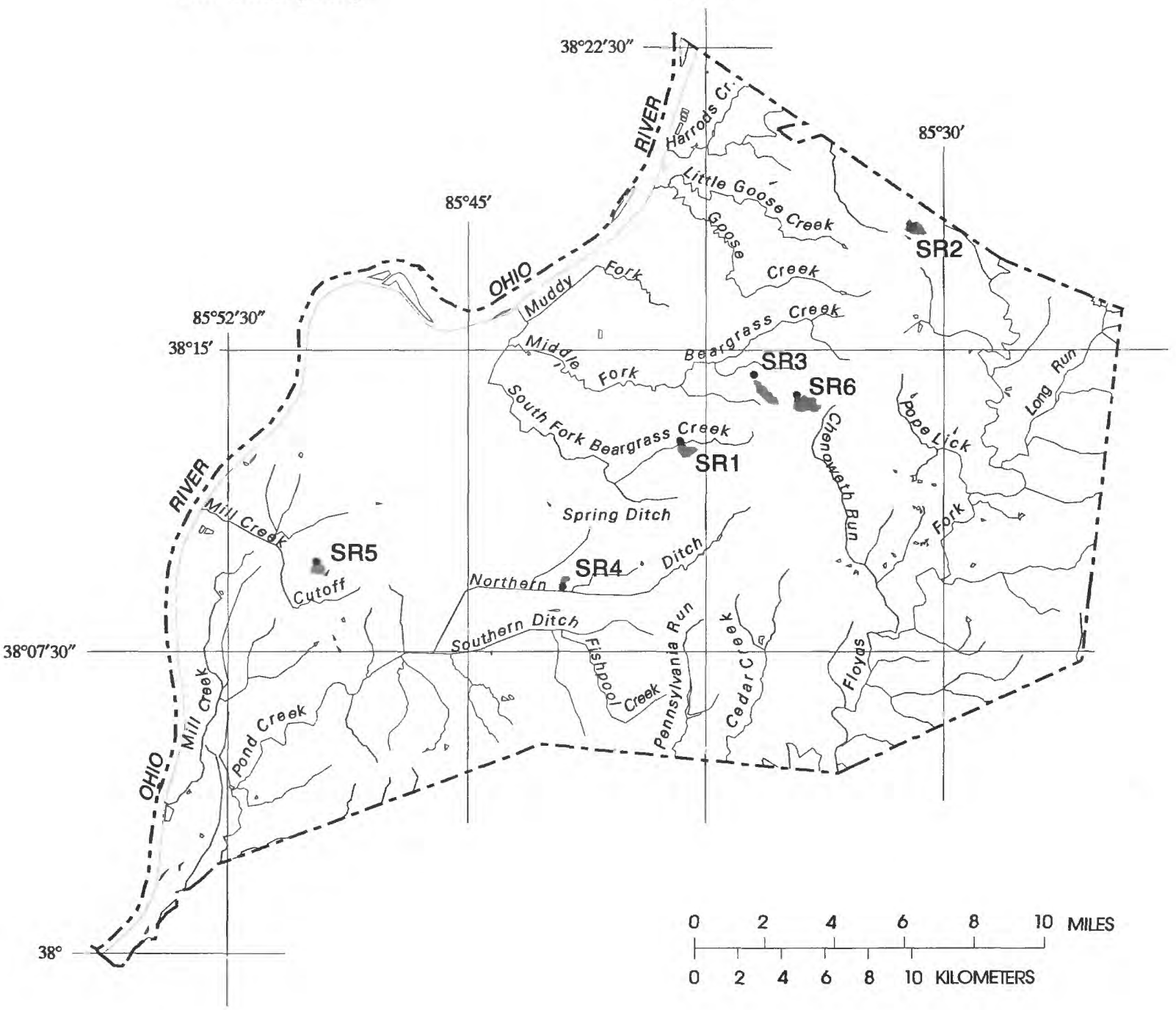

Base from U.S. Geological Survey, dlgltal data, 1:100,000, 1983 Universal Transverse Mercator projection, Zone 16

Figure 3.--Stormwater outfall sampling sites in Jefferson County, Kentucky. 
County are listed in table 3. Additional water-quality data, sampling procedures, analytical methods, and quality-assurance analyses are detailed in a data report by Evaldi and Moore (1992).

Table 2. Rainfall and runoff associated with stormwater samples collected in Jefferson County, Kentucky, January 1991-May 1992

$[-$, unknown $]$

\begin{tabular}{|c|c|c|c|c|c|}
\hline Site & $\begin{array}{l}\text { Date and time } \\
\text { rainfall began }\end{array}$ & $\begin{array}{l}\text { Total storm } \\
\text { rainfall, } \\
\text { in inches } \\
\end{array}$ & $\begin{array}{c}\text { Storm } \\
\text { duration, } \\
\text { in minutes }\end{array}$ & $\begin{array}{c}\text { Time since } \\
\text { previous rain, } \\
\text { in hours }\end{array}$ & $\begin{array}{l}\text { Total storm } \\
\text { runoff, in } \\
\text { cubic feet }\end{array}$ \\
\hline SR1 & $910115 @ 1650$ & 0.18 & 600 & 97.0 & - \\
\hline SR1 & $910419 @ 0320$ & .22 & 76 & 82.9 & 7,780 \\
\hline SR1 & $911005 @ 0458$ & .40 & 222 & 246.2 & 10,400 \\
\hline SR2 & $910205 @ 1056$ & 1.60 & 2339 & 146.1 & - - \\
\hline SR2 & $910419 @ 0320$ & .22 & 76 & 91.9 & 16,500 \\
\hline SR2 & 911005 @ 0455 & .44 & 223 & 246.5 & 16,900 \\
\hline SR3 & $910301 @ 1458$ & .22 & 362 & 268.6 & 14,800 \\
\hline SR3 & 910904 @ 0815 & 1.41 & 740 & 128.2 & 172,000 \\
\hline SR3 & $911212 @ 1222$ & .04 & 26 & 71.5 & 1,890 \\
\hline SR4 & $910312 @ 1325$ & .92 & 2,435 & 141.2 & 73,300 \\
\hline SR4 & $920212 @ 1426$ & .48 & 1,172 & 705.8 & 35,700 \\
\hline SR4 & 920317 @ 2105 & 1.74 & 2,495 & 181.2 & 478,000 \\
\hline SR4 & $920415 @ 0552$ & .57 & 381 & 109.5 & 30,900 \\
\hline SR5 & $910327 @ 1050$ & .03 & 20 & 116.0 & 11,000 \\
\hline SR5 & $910621 @ 1228$ & .92 & 132 & 18.4 & 105,000 \\
\hline SR5 & $920108 @ 1315$ & .05 & 85 & 123.5 & 100 \\
\hline SR5 & $920317 @ 2040$ & 2.54 & 2,540 & 181.0 & 379,000 \\
\hline SR5 & $920512 @ 1650$ & .61 & 530 & 74.3 & 24,700 \\
\hline SR6 & $910404 @ 1302$ & .13 & 452 & 310.2 & 3,260 \\
\hline SR6 & 910708 @ 1915 & .10 & 35 & 60.1 & 24,600 \\
\hline SR6 & $911212 @ 1149$ & .04 & 69 & 70.6 & 2,370 \\
\hline
\end{tabular}

${ }^{1}$ Date is given as year, month, day; time is given in 24-hour form.

Another network of sampling sites operated cooperatively by the MSD and USGS was in Jefferson County beginning in February 1988 (fig. 4, table 4). Unlike the six storm-outfall sampling sites, the sampling sites in this network are in receiving waters of the county. Water-quality data from this stream network for February 1988-March 1991 were summarized in a report by Evaldi and others (1993). The drainage area, the percentage of the area covered by impervious surfaces, and the percentage of selected land uses 
Table 3. Loads and event mean concentrations of selected constituents in storm runoff measured at stormwater outfalls in Jefferson County, Kentucky, January 1991-May 1992

[mg/L, milligrams per liter; 1b, pounds; --, missing. Censored data were set to the detection limit, and indicated loads and concentrations are maximum probable values]

\begin{tabular}{|c|c|c|c|c|c|c|c|c|c|c|}
\hline Site & $\begin{array}{l}\text { Date } \\
\text { storm } \\
\text { began }\end{array}$ & $\begin{array}{l}\text { Event } \\
\text { mean } \\
\text { concen- } \\
\text { tration, } \\
\text { (mg/L) }\end{array}$ & $\begin{array}{c}\text { Load for } \\
\text { first } \\
3 \\
\text { hours, } \\
\text { (1b) }\end{array}$ & $\begin{array}{l}\text { Total } \\
\text { storm } \\
\text { load, } \\
\text { (1b) }\end{array}$ & $\begin{array}{c}\text { Event } \\
\text { mean } \\
\text { concen- } \\
\text { tration, } \\
(\mathrm{mg} / \mathrm{L}) \\
\end{array}$ & $\begin{array}{c}\text { Load for } \\
\text { first } \\
3 \\
\text { hours, } \\
\text { (1b) } \\
\end{array}$ & $\begin{array}{l}\text { Total } \\
\text { storm } \\
\text { load, } \\
\text { (1b) }\end{array}$ & $\begin{array}{l}\text { Event } \\
\text { mean } \\
\text { concen- } \\
\text { tration, } \\
\text { (mg/L) } \\
\end{array}$ & $\begin{array}{c}\text { Load for } \\
\text { first } \\
3 \\
\text { hours, } \\
\text { (1b) }\end{array}$ & $\begin{array}{l}\text { Total } \\
\text { storm } \\
\text { load, } \\
\text { (lb) }\end{array}$ \\
\hline & & \multicolumn{3}{|c|}{ Chemical oxygen demand } & \multicolumn{3}{|c|}{ Biochemical oxygen demand } & \multicolumn{3}{|c|}{ Dissolved solids } \\
\hline $\begin{array}{l}\text { SR1 } \\
\text { SR1 } \\
\text { SR1 }\end{array}$ & $\begin{array}{l}910115 \\
910419 \\
911005\end{array}$ & $\begin{array}{c}30.0 \\
107 \\
82.0\end{array}$ & $\begin{array}{l}6.84 \\
51.7 \\
52.4\end{array}$ & $\begin{array}{c}-- \\
51.8 \\
53.2\end{array}$ & $\begin{array}{l}7.00 \\
-- \\
40.0\end{array}$ & $\begin{array}{c}1.60 \\
23.5\end{array}$ & $\begin{array}{c}-- \\
-- \\
25.7\end{array}$ & $\begin{array}{r}534 \\
64.6 \\
95.9\end{array}$ & $\begin{array}{r}122 \\
30.7 \\
48.3\end{array}$ & $\begin{array}{c}-- \\
31.4 \\
62.2\end{array}$ \\
\hline $\begin{array}{l}\text { SR2 } \\
\text { SR2 } \\
\text { SR2 }\end{array}$ & $\begin{array}{l}910205 \\
910419 \\
911005\end{array}$ & $\begin{array}{r}189 \\
62.0 \\
99.0\end{array}$ & $\begin{array}{l}65.3 \\
62.0 \\
97.9\end{array}$ & $\begin{array}{c}-\overline{63.9} \\
104\end{array}$ & $\begin{array}{c}71.0 \\
51.0\end{array}$ & $\begin{array}{l}24.5 \\
50.4\end{array}$ & $\begin{array}{c}-- \\
53.5\end{array}$ & $\begin{array}{r}484 \\
56.0 \\
60.0\end{array}$ & $\begin{array}{r}167 \\
56.0 \\
59.3\end{array}$ & $\begin{array}{c}-- \\
57.7 \\
62.9\end{array}$ \\
\hline $\begin{array}{l}\text { SR3 } \\
\text { SR3 } \\
\text { SR3 }\end{array}$ & $\begin{array}{l}910301 \\
910904 \\
911212\end{array}$ & $\begin{array}{r}94.6 \\
54.9 \\
119\end{array}$ & $\begin{array}{c}70.7 \\
159 \\
12.3\end{array}$ & $\begin{array}{c}87.3 \\
589 \\
-\end{array}$ & $\begin{array}{l}24.0 \\
13.0 \\
21.0\end{array}$ & $\begin{array}{c}15.7 \\
23.0 \\
2.18\end{array}$ & $\begin{array}{l}-- \\
-- \\
--\end{array}$ & $\begin{array}{l}296 \\
140 \\
580\end{array}$ & $\begin{array}{c}194 \\
264 \\
60.1\end{array}$ & $1,500_{--}^{--}$ \\
\hline $\begin{array}{l}\text { SR4 } \\
\text { SR4 } \\
\text { SR4 } \\
\text { SR4 }\end{array}$ & $\begin{array}{l}910312 \\
920212 \\
920317 \\
920415\end{array}$ & $\begin{array}{c}52.2 \\
159 \\
20.0 \\
66.1\end{array}$ & $\begin{array}{c}48.7 \\
45.4 \\
6.42 \\
79.4\end{array}$ & $\begin{array}{l}239 \\
-- \\
595 \\
127\end{array}$ & $\begin{array}{r}48.0 \\
147 \\
4.00 \\
1.00\end{array}$ & $\begin{array}{c}21.2 \\
41.9 \\
1.51 \\
.810\end{array}$ & $\begin{array}{l}-- \\
-- \\
--\end{array}$ & $\begin{array}{l}162 \\
240 \\
235 \\
258\end{array}$ & $\begin{array}{c}92.9 \\
104 \\
61.9 \\
282\end{array}$ & $\begin{array}{r}742 \\
537 \\
7,010 \\
496\end{array}$ \\
\hline $\begin{array}{l}\text { SR5 } \\
\text { SR5 } \\
\text { SR5 } \\
\text { SR5 } \\
\text { SR5 }\end{array}$ & $\begin{array}{l}910327 \\
910621 \\
920108 \\
920317 \\
920512\end{array}$ & $\begin{array}{c}365 \\
26.0 \\
-- \\
29.9 \\
39.0\end{array}$ & $\begin{array}{c}238 \\
150 \\
-- \\
27.3 \\
41.0\end{array}$ & $\begin{array}{c}251 \\
168 \\
-- \\
708 \\
60.1\end{array}$ & $\begin{array}{l}610 \\
26.0 \\
20.0 \\
96.0 \\
36.0\end{array}$ & $\begin{array}{c}136 \\
150 \\
.120 \\
16.3 \\
21.1\end{array}$ & $\begin{array}{c}168 \\
.120 \\
-- \\
--\end{array}$ & $\begin{array}{r}174 \\
167 \\
300 \\
135 \\
57.4\end{array}$ & $\begin{array}{c}50.7 \\
1,000 \\
1.87 \\
8.13 \\
44.6\end{array}$ & $\begin{array}{c}120 \\
1,100 \\
1.87 \\
3,200 \\
88.5\end{array}$ \\
\hline $\begin{array}{l}\text { SR6 } \\
\text { SR6 } \\
\text { SR6 }\end{array}$ & $\begin{array}{l}910404 \\
910708 \\
911212\end{array}$ & $\begin{array}{l}407 \\
140 \\
359\end{array}$ & $\begin{array}{r}81.2 \\
215 \\
51.7\end{array}$ & $\begin{array}{c}82.7 \\
215 \\
-\end{array}$ & $\begin{array}{c}-- \\
45.0 \\
73.0\end{array}$ & $\begin{array}{c}-- \\
69.0 \\
10.5\end{array}$ & 69.0 & $\begin{array}{l}152 \\
264 \\
144\end{array}$ & $\begin{array}{c}30.3 \\
405 \\
20.7\end{array}$ & $\begin{array}{c}30.9 \\
405 \\
-\end{array}$ \\
\hline
\end{tabular}

Suspended solids

\begin{tabular}{lcccc} 
SR1 & 910115 & 36.0 & 8.21 & -- \\
SR1 & 910419 & 47.6 & 23.0 & 23.1 \\
SR1 & 911005 & 40.8 & 25.9 & 26.5 \\
& & & & \\
SR2 & 910205 & 300 & 104 & -- \\
SR2 & 910419 & 24.0 & 24.0 & 24.7 \\
SR2 & 911005 & 202 & 200 & 212 \\
& & & & \\
SR3 & 910301 & 248 & 162 & -- \\
SR3 & 910904 & 46.8 & 90.2 & 502 \\
SR3 & 911212 & 272 & 28.2 & -- \\
& & & & \\
SR4 & 910312 & 44.8 & 77.0 & 205 \\
SR4 & 920212 & 43.4 & 22.8 & 96.8 \\
SR4 & 920317 & 47.9 & 15.9 & 1,430 \\
SR4 & 920415 & 61.7 & 81.0 & 119 \\
& & & & \\
SR5 & 910327 & 63.8 & 17.8 & 43.8 \\
SR5 & 910621 & 118 & 762 & 778 \\
SR5 & 920108 & 76.0 & \multicolumn{1}{c}{.470} & .470 \\
SR5 & 920317 & 48.0 & 8.47 & 1,140 \\
SR5 & 920512 & 19.1 & 19.9 & 29.5 \\
& & & & \\
SR6 & 910404 & 72.0 & 14.4 & 14.6 \\
SR6 & 910708 & 126 & 193 & 193 \\
SR6 & 911212 & 112 & 16.1 & --
\end{tabular}

\section{Total nitrogen}

$2.70 \quad 0.616 \quad--$

3.45

1.98

3.06

1.89
2.95

1.14

1.29

1.06

1.89

2.92

$--$

2.48

1.73

1.62

2.49

3.09

5.49

.569

18.

2.41

1.62

1.06
1.63

1.07

.868

1.54

$--$

1.87

3.20

3.20
1.47

2.02

2.07

1.98
$.596 \quad 1.29$

$-\therefore$

1. 59

3.11

.413

3.04

.421

3.04

\section{Total Kjeldahl nitrogen}

$\begin{array}{lll}1.29 & 0.294 & -- \\ 2.42 & 1.17 & 1.18 \\ 1.26 & .700 & .820 \\ 1.93 & .667 & -- \\ 1.04 & 1.04 & 1.07 \\ 2.05 & 2.03 & 2.15 \\ 1.70 & 1.11 & -- \\ .994 & 1.17 & 10.6 \\ 1.59 & .165 & -- \\ & & \\ .393 & .434 & 1.80 \\ .753 & .434 & 1.68 \\ .360 & .143 & 10.7 \\ .871 & .875 & 1.68 \\ 1.12 & .480 & .768 \\ .476 & 2.65 & 3.13 \\ 1.76 & .011 & .011 \\ .600 & .103 & 14.2 \\ 1.21 & .985 & 1.86 \\ 1.49 & .297 & .303 \\ 1.51 & 2.32 & 2.32 \\ -. & -- & --\end{array}$


Table 3. Loads and event mean concentrations of selected constituents in storm runoff measured at stormwater outfalls in Jefferson County, Kentucky, January 1991-May 1992--Continued

[mg/L, milligrams per liter; lb, pounds; --, missing. Censored data were set to the detection limit, and indicated loads and concentrations are maximum probable values]

\begin{tabular}{|c|c|c|c|c|c|c|c|c|c|c|}
\hline Site & $\begin{array}{l}\text { Date } \\
\text { storm } \\
\text { began } 1\end{array}$ & $\begin{array}{l}\text { Event } \\
\text { mean } \\
\text { concen- } \\
\text { tration, } \\
(\mathrm{mg} / \mathrm{L})\end{array}$ & $\begin{array}{c}\text { Load for } \\
\text { first } \\
3 \\
\text { hours, } \\
\text { (1b) }\end{array}$ & $\begin{array}{l}\text { Total } \\
\text { storm } \\
\text { load, } \\
\text { (lb) }\end{array}$ & $\begin{array}{l}\text { Event } \\
\text { mean } \\
\text { concen- } \\
\text { tration, } \\
(\mathrm{mg} / \mathrm{L})\end{array}$ & $\begin{array}{c}\text { Load for } \\
\text { first } \\
3 \\
\text { hours, } \\
\text { (1b) }\end{array}$ & $\begin{array}{l}\text { Total } \\
\text { storm } \\
\text { load, } \\
\text { (lib) }\end{array}$ & $\begin{array}{l}\text { Event } \\
\text { mean } \\
\text { concen- } \\
\text { tration, } \\
(\mathrm{mg} / \mathrm{L})\end{array}$ & $\begin{array}{c}\text { Load for } \\
\text { first } \\
3 \\
\text { hours, } \\
(1 \mathrm{~b}) \\
\end{array}$ & $\begin{array}{l}\text { Total } \\
\text { storm } \\
\text { load, } \\
\text { (1b) }\end{array}$ \\
\hline & & \multicolumn{3}{|c|}{ Total phosphorus } & \multicolumn{3}{|c|}{ Dissolved phosphorus } & \multicolumn{3}{|c|}{ Total cadmium } \\
\hline $\begin{array}{l}\text { SR1 } \\
\text { SR1 } \\
\text { SR1 }\end{array}$ & $\begin{array}{l}910115 \\
910419 \\
911005\end{array}$ & $\begin{array}{r}0.250 \\
.460 \\
1.19\end{array}$ & $\begin{array}{r}0.060 \\
.220 \\
.770\end{array}$ & $\begin{array}{l}-- \\
0.220 \\
.770\end{array}$ & $\begin{array}{l}0.310 \\
1.10\end{array}$ & $\begin{array}{r}-. \\
0.148 \\
.648\end{array}$ & $\begin{array}{r}-. \\
0.151 \\
.708\end{array}$ & $\begin{array}{r}0.007 \\
.007 \\
.007\end{array}$ & $\begin{array}{r}0.002 \\
.003 \\
.004\end{array}$ & $\begin{array}{l}-- \\
0.003 \\
.005\end{array}$ \\
\hline $\begin{array}{l}\text { SR2 } \\
\text { SR2 } \\
\text { SR2 }\end{array}$ & $\begin{array}{l}910205 \\
910419 \\
911005\end{array}$ & $\begin{array}{l}.810 \\
.390 \\
.260\end{array}$ & $\begin{array}{l}.280 \\
.390 \\
.260\end{array}$ & $\begin{array}{r}.400 \\
.270\end{array}$ & $\begin{array}{r}-. \\
.450 \\
.200\end{array}$ & $\begin{array}{r}.450 \\
.198\end{array}$ & $\begin{array}{r}.464 \\
.210\end{array}$ & $\begin{array}{l}.007 \\
.007 \\
.007\end{array}$ & $\begin{array}{l}.002 \\
.007 \\
.007\end{array}$ & $\begin{array}{l}.007 \\
.007\end{array}$ \\
\hline $\begin{array}{l}\text { SR3 } \\
\text { SR3 } \\
\text { SR3 }\end{array}$ & $\begin{array}{l}910301 \\
910904 \\
911212\end{array}$ & $\begin{array}{l}.080 \\
.220 \\
.370\end{array}$ & $\begin{array}{l}.050 \\
.340 \\
.040\end{array}$ & 2.30 & $\begin{array}{l}.050 \\
.120 \\
.030\end{array}$ & $\begin{array}{l}.033 \\
.212 \\
.003\end{array}$ & $\begin{array}{l}-- \\
-- \\
--\end{array}$ & $\begin{array}{l}.008 \\
.007 \\
.007\end{array}$ & $\begin{array}{l}.005 \\
.012 \\
.001\end{array}$ & $\begin{array}{r}.007 \\
.075 \\
--\end{array}$ \\
\hline $\begin{array}{l}\text { SR4 } \\
\text { SR4 } \\
\text { SR4 } \\
\text { SR4 }\end{array}$ & $\begin{array}{l}910312 \\
920212 \\
920317 \\
920415\end{array}$ & $\begin{array}{l}.190 \\
.560 \\
.120 \\
.080\end{array}$ & $\begin{array}{l}.080 \\
.060 \\
.030 \\
.050\end{array}$ & $\begin{array}{l}-- \\
1.25 \\
3.56 \\
\quad .160\end{array}$ & $\begin{array}{l}.170 \\
.170 \\
--\end{array}$ & $\begin{array}{l}.075 \\
.049 \\
--\end{array}$ & $\begin{array}{l}-- \\
-- \\
-- \\
--\end{array}$ & $\begin{array}{l}.007 \\
.007 \\
.007 \\
.023\end{array}$ & $\begin{array}{l}.003 \\
.002 \\
.003 \\
.033\end{array}$ & $\begin{array}{l}.032 \\
.016 \\
.209 \\
.044\end{array}$ \\
\hline $\begin{array}{l}\text { SR5 } \\
\text { SR5 } \\
\text { SR5 } \\
\text { SR5 } \\
\text { SR5 }\end{array}$ & $\begin{array}{l}910327 \\
910621 \\
920108 \\
920317 \\
920512\end{array}$ & $\begin{array}{l}.100 \\
.180 \\
.380 \\
.250 \\
.120\end{array}$ & $\begin{array}{l}.020 \\
1.15 \\
-- \\
.020 \\
.070\end{array}$ & $\begin{array}{l}.070 \\
1.21 \\
5.89 \\
.180\end{array}$ & $\begin{array}{c}.070 \\
.150 \\
-- \\
-.090\end{array}$ & $\begin{array}{c}.016 \\
.865 \\
-- \\
- \\
.053\end{array}$ & $\begin{array}{l}-. \\
-.971 \\
-- \\
--\end{array}$ & $\begin{array}{l}.009 \\
.008 \\
.007 \\
.007 \\
.007\end{array}$ & $\begin{array}{l}.002 \\
.040 \\
- \\
.001 \\
.004\end{array}$ & $\begin{array}{r}.006 \\
.050 \\
-. \\
.166 \\
.011\end{array}$ \\
\hline $\begin{array}{l}\text { SR6 } \\
\text { SR6 } \\
\text { SR6 }\end{array}$ & $\begin{array}{l}910404 \\
910708 \\
911212\end{array}$ & $\begin{array}{l}.370 \\
.190 \\
.180\end{array}$ & $\begin{array}{l}.070 \\
.290 \\
.030\end{array}$ & $\begin{array}{r}.080 \\
.290 \\
--\end{array}$ & $\begin{array}{l}.010 \\
.110 \\
.050\end{array}$ & $\begin{array}{l}.002 \\
.169 \\
.007\end{array}$ & $\begin{array}{r}.002 \\
.169 \\
--\end{array}$ & $\begin{array}{l}.035 \\
.007 \\
.007\end{array}$ & $\begin{array}{l}.007 \\
.011 \\
.001\end{array}$ & $\begin{array}{r}.007 \\
.011 \\
--\end{array}$ \\
\hline & & \multicolumn{3}{|c|}{ Total copper } & \multicolumn{3}{|c|}{ Total lead } & \multicolumn{3}{|c|}{ Total zinc } \\
\hline $\begin{array}{l}\text { SR1 } \\
\text { SR1 } \\
\text { SR1 }\end{array}$ & $\begin{array}{l}910115 \\
910419 \\
911005\end{array}$ & $\begin{array}{r}0.014 \\
.009 \\
.008\end{array}$ & $\begin{array}{r}0.003 \\
.004 \\
.005\end{array}$ & $\begin{array}{r}0.004 \\
.005\end{array}$ & $\begin{array}{r}0.055 \\
.060 \\
.060\end{array}$ & $\begin{array}{r}0.013 \\
.029 \\
.035\end{array}$ & $\begin{array}{r}0.029 \\
.039\end{array}$ & $\begin{array}{r}0.162 \\
.362 \\
.066\end{array}$ & $\begin{array}{r}0.037 \\
.175 \\
.036\end{array}$ & $\begin{array}{l}-- \\
0.176 \\
.043\end{array}$ \\
\hline $\begin{array}{l}\text { SR2 } \\
\text { SR2 } \\
\text { SR2 }\end{array}$ & $\begin{array}{l}910205 \\
910419 \\
911005\end{array}$ & $\begin{array}{l}.017 \\
.008 \\
.008\end{array}$ & $\begin{array}{l}.006 \\
.008 \\
.008\end{array}$ & $\begin{array}{r}.008 \\
.008\end{array}$ & $\begin{array}{l}.055 \\
.060 \\
.060\end{array}$ & $\begin{array}{l}.019 \\
.060 \\
.059\end{array}$ & $\begin{array}{r}.062 \\
.063\end{array}$ & $\begin{array}{l}.142 \\
.157 \\
.529\end{array}$ & $\begin{array}{l}.049 \\
.157 \\
.523\end{array}$ & $\begin{array}{l}.162 \\
.555\end{array}$ \\
\hline $\begin{array}{l}\text { SR3 } \\
\text { SR3 } \\
\text { SR3 }\end{array}$ & $\begin{array}{l}910301 \\
910904 \\
911212\end{array}$ & $\begin{array}{l}.016 \\
.015 \\
.037\end{array}$ & $\begin{array}{l}.011 \\
.021 \\
.004\end{array}$ & $\begin{array}{r}.015 \\
.164 \\
--\end{array}$ & $\begin{array}{l}.055 \\
.060 \\
.060\end{array}$ & $\begin{array}{l}.036 \\
.106 \\
.006\end{array}$ & $\begin{array}{r}.051 \\
.643 \\
--\end{array}$ & $\begin{array}{l}.103 \\
.045 \\
.187\end{array}$ & $\begin{array}{l}.079 \\
.126 \\
.019\end{array}$ & $\begin{array}{r}.095 \\
.483 \\
-.\end{array}$ \\
\hline $\begin{array}{l}\text { SR4 } \\
\text { SR4 } \\
\text { SR4 } \\
\text { SR4 }\end{array}$ & $\begin{array}{l}910312 \\
920212 \\
920317 \\
920415\end{array}$ & $\begin{array}{l}.012 \\
.013 \\
.013 \\
.019\end{array}$ & $\begin{array}{l}.008 \\
.005 \\
.008 \\
.020\end{array}$ & $\begin{array}{l}.053 \\
.028 \\
.390 \\
.036\end{array}$ & $\begin{array}{l}.067 \\
.055 \\
.055 \\
.053\end{array}$ & $\begin{array}{l}.058 \\
.016 \\
.021 \\
.043\end{array}$ & $\begin{array}{c}.306 \\
.123 \\
1.64 \\
.102\end{array}$ & $\begin{array}{l}.215 \\
.125 \\
.068 \\
.238\end{array}$ & $\begin{array}{l}.093 \\
.058 \\
.037 \\
.139\end{array}$ & $\begin{array}{r}.983 \\
.280 \\
2.04 \\
.459\end{array}$ \\
\hline $\begin{array}{l}\text { SR5 } \\
\text { SR5 } \\
\text { SR5 } \\
\text { SR5 } \\
\text { SR5 }\end{array}$ & $\begin{array}{l}910327 \\
910621 \\
920108 \\
920317 \\
920512\end{array}$ & $\begin{array}{l}.017 \\
.008 \\
.021 \\
.016 \\
.015\end{array}$ & $\begin{array}{r}.006 \\
.046 \\
-.003 \\
.009\end{array}$ & $\begin{array}{r}.011 \\
.053 \\
-- \\
.379 \\
.023\end{array}$ & $\begin{array}{l}.060 \\
.060 \\
.055 \\
.055 \\
.053\end{array}$ & $\begin{array}{r}.013 \\
.346 \\
-- \\
.009 \\
.031\end{array}$ & $\begin{array}{c}.041 \\
.394 \\
-- \\
1.30 \\
.082\end{array}$ & $\begin{array}{l}.235 \\
.100 \\
.330 \\
.066 \\
.144\end{array}$ & $\begin{array}{l}.105 \\
.594 \\
.002 \\
.023 \\
.118\end{array}$ & $\begin{array}{r}.162 \\
.658 \\
.002 \\
1.57 \\
.223\end{array}$ \\
\hline $\begin{array}{l}\text { SR6 } \\
\text { SR6 } \\
\text { SR6 }\end{array}$ & $\begin{array}{l}910404 \\
910708 \\
911212\end{array}$ & $\begin{array}{l}.025 \\
.010 \\
.012\end{array}$ & $\begin{array}{l}.005 \\
.015 \\
.002\end{array}$ & $\begin{array}{r}.005 \\
.015 \\
-\end{array}$ & $\begin{array}{l}.055 \\
.060 \\
.060\end{array}$ & $\begin{array}{l}.011 \\
.092 \\
.009\end{array}$ & $\begin{array}{r}.011 \\
.092 \\
--\end{array}$ & $\begin{array}{l}.256 \\
.324 \\
.211\end{array}$ & $\begin{array}{l}.051 \\
.497 \\
.030\end{array}$ & $\begin{array}{r}.052 \\
-.497\end{array}$ \\
\hline
\end{tabular}

${ }^{1}$ Date is given as year, month, day. 


\section{EXPLANATION}

\section{$\nabla^{17}$ Water-quality sampling site and identifier (see table 3 for site description)}

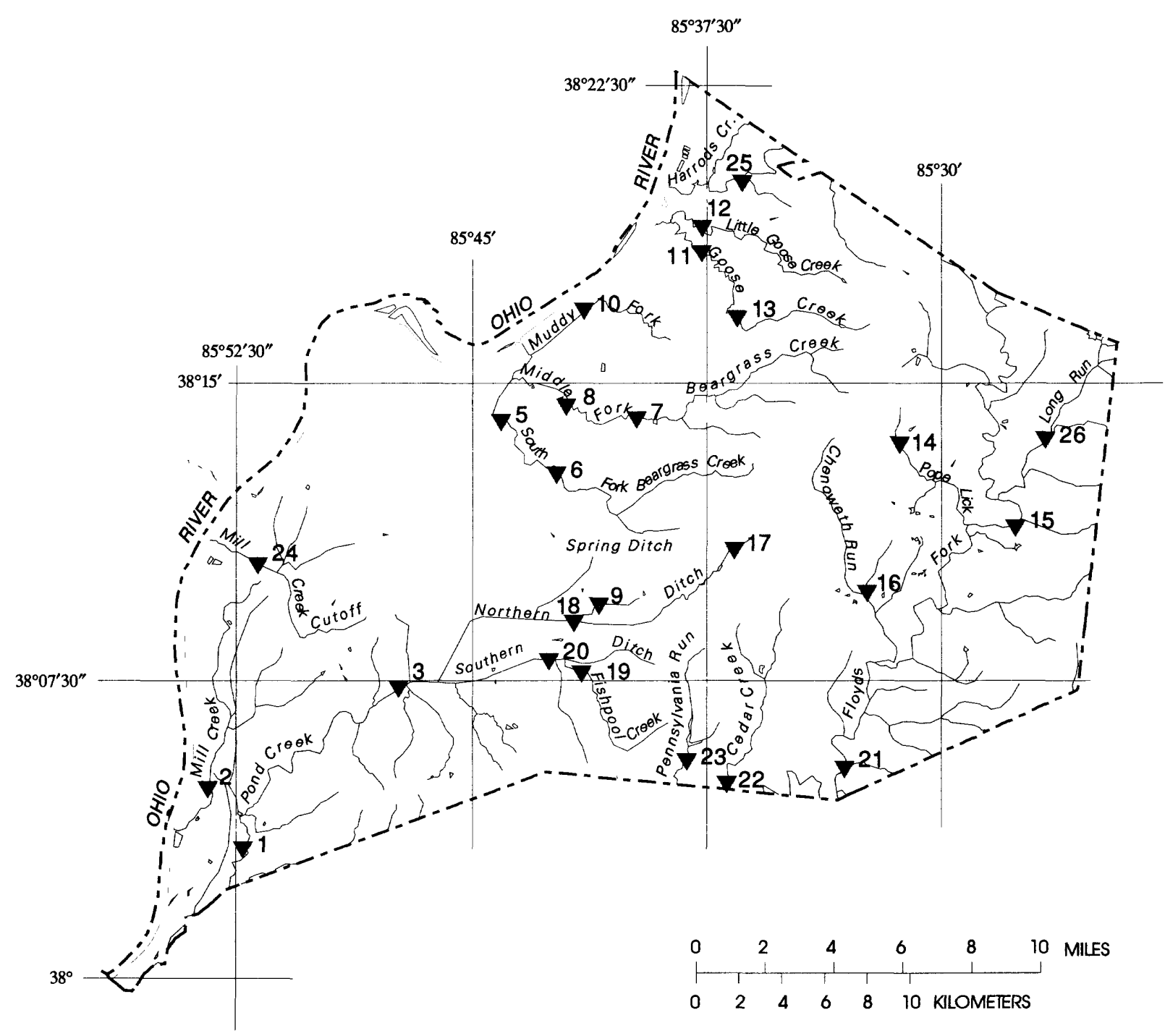

Base from U.S. Geologlcal Survey, dlgltal data, 1:100,000, 1983 Universal Transverse Mercator projectlon, Zone 16

Figure 4.--Stream-water-quality sampling sites in Jefferson County, Kentucky. 
Table 4. Stream-water-quality sampling sites in Jefferson County, Kentucky, and potential sources of contaminants in their watersheds

[NPR, nonpoint runoff; WTE, wastewater-treatment-plant effluent; STD, septic-tank discharges; IW, industrial wastewater; CSO, combined-sewer or sanitary-sewer overflows]

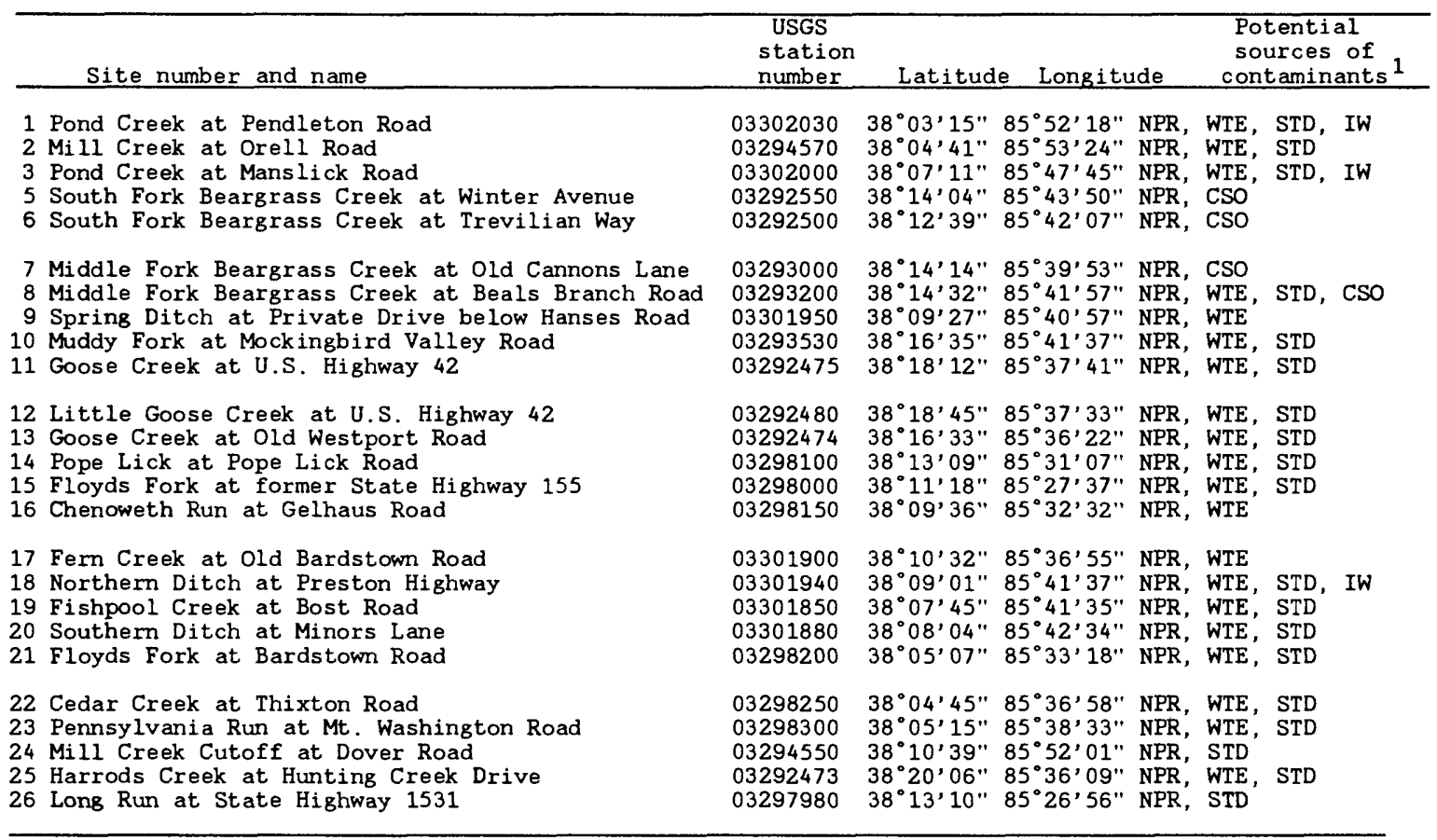

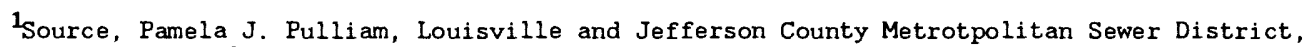
written commun., 1992 . 
within the drainage basin are listed in table 5 for the 6 stormwater-outfall and 25 stream-water-quality sampling sites in Jefferson County. These data are needed in some load-estimation procedures.

\section{TECHNIQUES FOR ESTIMATING THE QUANTITY AND QUALITY OF STORM RUNOFF}

Regional regression models to estimate storm-runoff volumes, mean constituent concentrations, and constituent loads were developed by Driver and Tasker (1990). Storm-runoff volumes, mean concentrations, and loads measured in Jefferson County were used to evaluate these regional estimation methods. Adjustments to some of the previously developed estimation models were based on Jefferson County measurements. In addition, models were developed from these data by use of multiple-regression analysis to relate storm-runoff constituent concentrations, volumes, and loads to measurable physical, landuse, and climatic characteristics in Jefferson County.

The previously developed regional regression models were of the same type as those developed during this study. The storm-runoff constituent concentrations, volumes, and loads were the response variables. Physical, land-use, and climatic characteristics were the explanatory variables and consisted of the following:

1. Total contributing drainage area.

2. Impervious area, as a percentage of total contributing drainage area.

3. Industrial land use, as a percentage of total contributing drainage area.

4. Commercial land use, as a percentage of total contributing drainage area.

5. Residential land use, as a percentage of total contributing drainage area.

6. Nonurban land use, as a percentage of total contributing drainage area.

7. Total storm or annual rainfall, in inches.

8. Mean annual rainfall, in inches.

9. Rainfall duration, in minutes.

10. Maximum 24-hour precipitation that has a 2-year recurrence interval, in inches.

11. Mean annual nitrogen load in precipitation, in pounds of nitrogen per acre.

12. Mean minimum January temperature, in degrees Fahrenheit.

\section{Loads of Constituents in Storm Runoff, and Quantity of Runoff}

National regression models developed by Driver and Tasker (1990) can be used to estimate loads of selected constituents and runoff quantity from single storms on the basis of total rainfall of the storm and on physical, land use, and climate characteristics of the drainage basin. Three regions of the United States were defined by Driver and Tasker (1990) on the basis of the amounts of mean annual precipitation in each region, and sets of models were developed for each region. The models used for estimation of loads of selected constituents in single storms and runoff quantity of the storms in Jefferson County were developed from urban storm-runoff data for 11 metropolitan areas in which mean annual rainfall is equal to or greater than 40 in. The closest of these 11 metropolitan areas was Knoxville, Tenn.; 


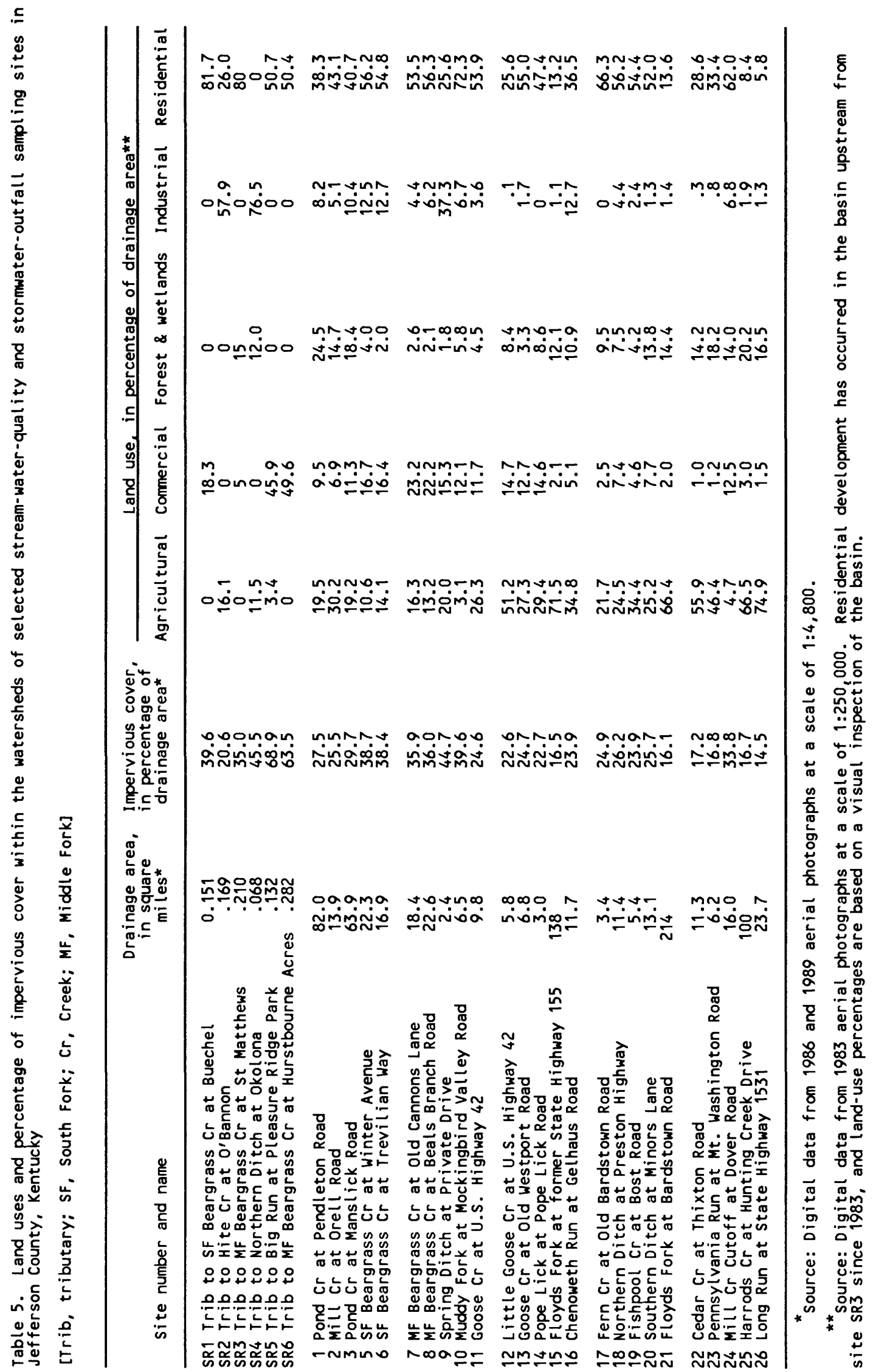


none were in Kentucky. Estimated runoff quantity and loads of selected constituents in single storms based on the regional regression models of Driver and Tasker (1990) were compared to runoff quantity (table 2) and constituent loads (table 3 ) measured at selected stormwater outfalls in Jefferson County to evaluate the reliability of the regional estimation models for use in the county.

Storms were defined by Driver and Tasker (1990) as rainfall events in which the total rainfall was at least 0.05 in. Three samples of Jefferson County runoff were collected during storms of less than 0.05 in. rainfall (table 2). Only the sample collected at Site SR5 on March 27, 1991, was not used in evaluating the regional regression models because the ratio of runoff to total rainfall indicated that the total measured rainfall may have been inaccurate.

Plots of the estimated and measured storm runoff and constituent loads in the runoff indicated that adjustments were needed in the estimation models. Adjustments were determined by simple linear regression between the estimated runoff quantity and constituent loads in the runoff and the Jefferson County measurements, with the regression intercepts set to zero (table 6). The means of the residuals of the regional-model estimates as compared to Jefferson County measurements were reduced after the adjustments. Regional regression models to estimate loads of biochemical oxygen demand and cadmium for single storms were not available.

Table 6. Adjustments to regional regression models for estimating loads of selected constituents in storm runoff and quantity of storm runoff in single storms from urban watersheds of Jefferson County, Kentucky

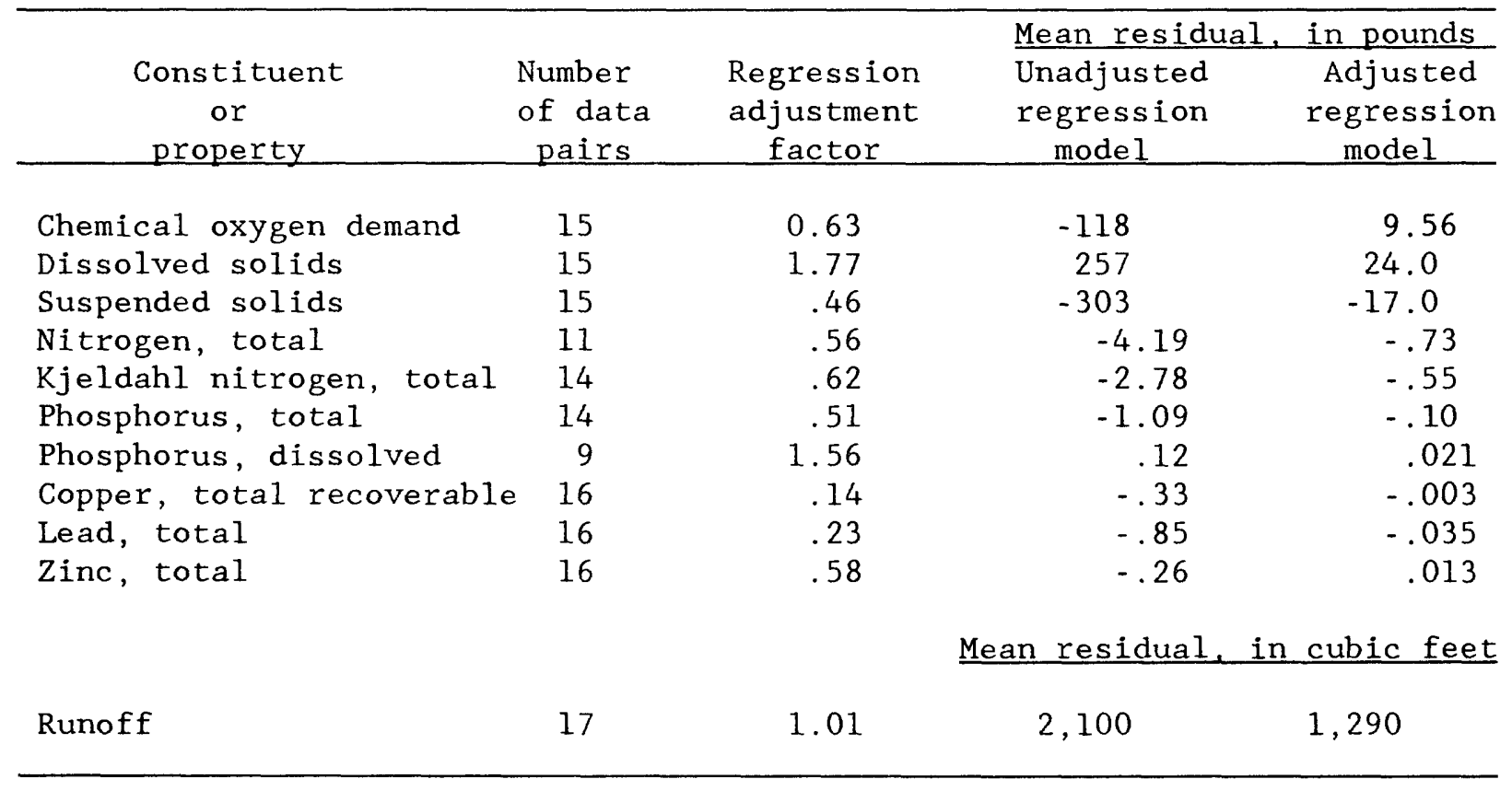


The drainage areas of the urban watersheds used to develop the regional models ranged from 0.014 to $0.830 \mathrm{mi}^{2}$. Watersheds whose drainage areas are greater than $0.5 \mathrm{mi}^{2}$ are calculated as though the drainage areas were $0.2 \mathrm{mi}^{2}$, and loads are then adjusted on the basis of actual drainage area. This method of estimation is based on the assumption that the estimated constituent loads are conservative and cumulative, an assumption that may not always be valid (Tasker and others, 1990). The adjusted regional regression models that can be used to compute loads in storm runoff and the quantity of runoff for single storms from urban watersheds of Jefferson County are

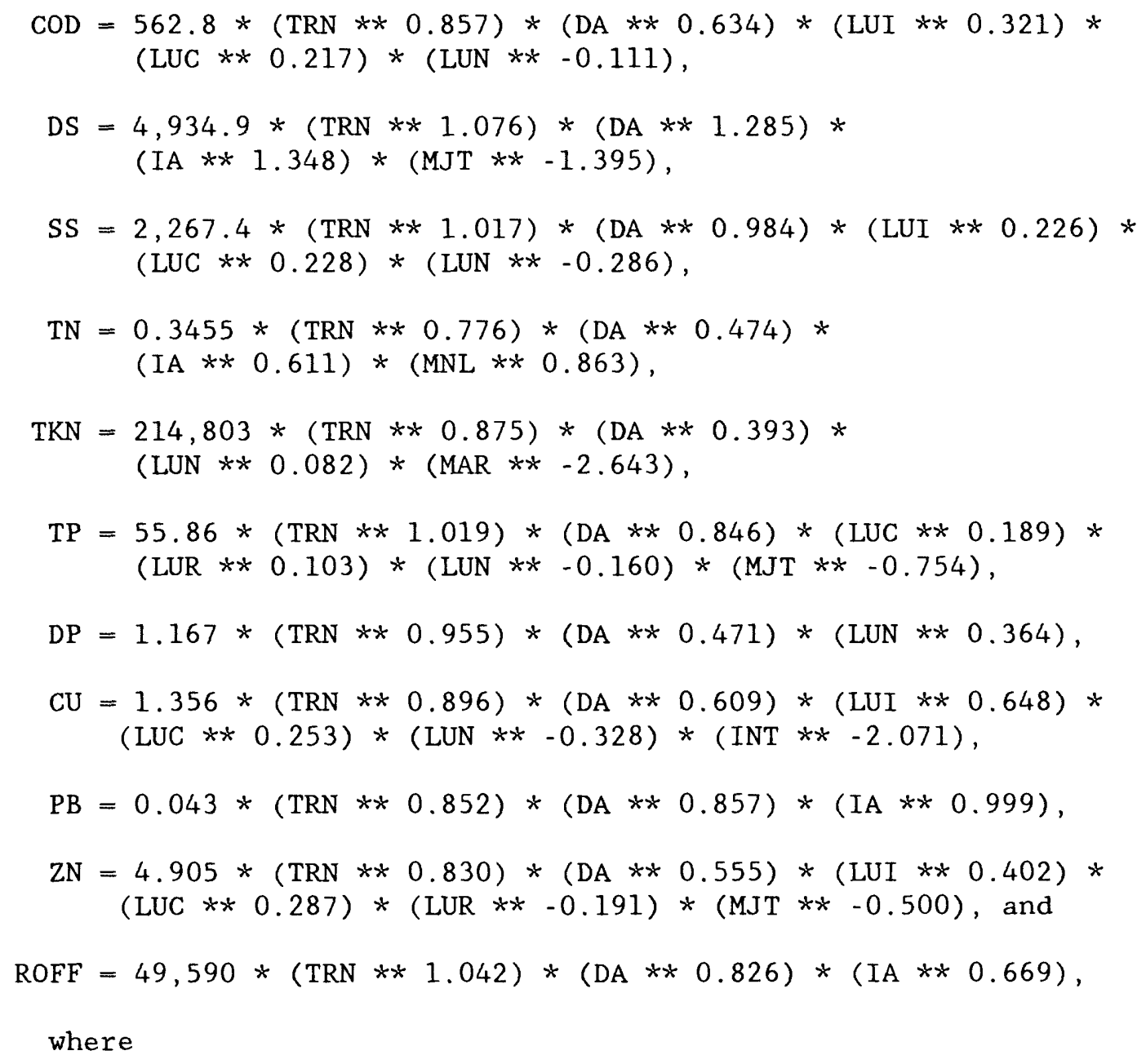

where

COD is load of chemical oxygen demand in storm runoff, in pounds;

DS is load of dissolved solids in storm runoff, in pounds;

SS is load of suspended solids in storm runoff, in pounds;

$\mathrm{TN}$ is load of total nitrogen in storm runoff, in pounds;

TKN is load of total Kjeldahl nitrogen in storm runoff, in pounds;

TP is load of total phosphorus in storm runoff, in pounds;

DP is load of dissolved phosphorus in storm runoff, in pounds;

$\mathrm{CU}$ is load of total copper in storm runoff, in pounds; 
$P B$ is load of total lead in storm runoff, in pounds;

$\mathrm{ZN}$ is load of total zinc in storm runoff, in pounds;

ROFF is storm runoff, in cubic feet;

TRN is total storm rainfall, in inches;

DA is drainage area, in square miles;

LUI is 1 + industrial land use, in percentage of drainage area;

LUC is $1+$ commercial land use, in percentage of drainage area;

LUN is $2+$ nonurban land use, in percentage of drainage area;

IA is 1 + impervious area, in percentage of drainage area;

MJT is mean minimum January temperature $\left(26.2^{\circ} \mathrm{F}\right.$, National Oceanic and Atmospheric Administration, 1990);

MNL is mean annual nitrogen load in precipitation (9 $1 \mathrm{~b}$ per acre, based on estimated load from precipitation of ammonia and nitrate; Evaldi and others, 1993);

MAR is mean annual rainfall (43.0 in., National Oceanic and Atmospheric Administration, 1990);

LUR is 1 + residential land use, in percentage of drainage area; and

INT is maximum 24-hour precipitation that has a 2-year recurrence interval (3.1 in.; Melcher and Ruhl, 1984).

Driver and Tasker (1990) also developed three-variable regression models to estimate loads of selected constituents in single storms that are simplified alternatives to the previously presented regression models 1-10. The explanatory variables are always (1) total storm rainfall, (2) drainage area, and (3) impervious area. The model for estimating the quantity of runoff of a single storm (equation 11) was already a three-variable model of this type. As for the original regional regression models, estimated constituent loads in storm runoff based on the three-variable regression models were compared to constituent loads measured in storm runoff at sites in Jefferson County to evaluate the accuracy of the three variable estimation models for use in the county. Again, plots of the estimated and measured constituent loads in storm runoff indicated that adjustments were needed in some of the three-variable estimation models. Adjustments were determined by simple linear regression between the estimated and measured constituent loads, with the regression intercepts set to zero (table 7). The mean of the residuals of the estimated and measured regression relations were reduced after the adjustments. 
Table 7. Adjustments to three-variable regional regression models for estimating loads of selected constituents in storm runoff in single storms from urban watersheds of Jefferson County, Kentucky

\begin{tabular}{lcccc}
\hline $\begin{array}{c}\text { Constituent } \\
\text { or } \\
\text { property }\end{array}$ & $\begin{array}{c}\text { Number } \\
\text { of data } \\
\text { pairs }\end{array}$ & $\begin{array}{c}\text { Regression } \\
\text { adjustment } \\
\text { factor }\end{array}$ & $\begin{array}{c}\text { Mean residual, in pounds } \\
\text { Unadjusted } \\
\text { regression } \\
\text { model }\end{array}$ & $\begin{array}{c}\text { Adjusted } \\
\text { regression } \\
\text { model }\end{array}$ \\
\hline Chemical oxygen demand & 15 & 0.58 & -120 & 3.46 \\
Dissolved solids & 15 & .63 & -142 & 118 \\
Suspended solids & 15 & .37 & -391 & -1.49 \\
Nitrogen, total & 11 & 1.00 & -.80 & -.80 \\
Kjeldahl nitrogen, total & 14 & .89 & -.97 & -.53 \\
Phosphorus, total & 14 & .77 & -.14 & -.006 \\
Phosphorus, dissolved & 9 & 1.98 & -.12 & -.021 \\
Copper, total recoverable & 16 & .43 & -.06 & -.034 \\
Lead, total & 16 & .19 & & .057 \\
Zinc, total & 16 & .41 & & -1.06 \\
\hline
\end{tabular}

Regression models 1-10 should be used instead of models 12-21 if all explanatory variables are available. The drainage areas of the urban watersheds used to develop the regional models ranged from 0.014 to $0.830 \mathrm{mi}^{2}$. Watersheds whose drainage areas are greater than $0.5 \mathrm{mi}^{2}$ are calculated as though the drainage areas are $0.2 \mathrm{mi}^{2}$, and loads are then adjusted on the basis of actual drainage area. This method of estimation is based on the assumption that the estimated constituent loads are conservative and cumulative, an assumption that may not always be valid (Tasker and others, 1990). The adjusted three-variable regional regression models that can be used to compute constituent loads in single storms from urban watersheds of Jefferson County are

$$
\begin{aligned}
& \mathrm{COD}=117.0 *(\mathrm{TRN} * * 0.851) *(\mathrm{DA} * * 0.601) *(\mathrm{IA} * * 0.528), \\
& \mathrm{DS}=2.945 *(\mathrm{TRN} * * 1.251) *(\mathrm{DA} * * 1.218) *(\mathrm{IA} * * 1.964), \\
& \mathrm{SS}=101.9 *(\mathrm{TRN} * * 1.002) *(\mathrm{DA} * * 1.009) *(\mathrm{IA} * * 0.837), \\
& \mathrm{TN}=3.063 *(\mathrm{TRN} * * 0.703) *(\mathrm{DA} * * 0.465) *(\mathrm{IA} * * 0.521), \\
& \mathrm{TKN}=5.833 *(\mathrm{TRN} * * 0.808) *(\mathrm{DA} * * 0.415) *(\mathrm{IA} * * 0.199), \\
& \mathrm{TP}=2.799 *(\mathrm{TRN} * * 0.954) *(\mathrm{DA} * * 0.789) *(\mathrm{IA} * * 0.289), \\
& \mathrm{DP}=9.711 *(\mathrm{TRN} * * 1.003) *(\mathrm{DA} * * 0.280) *(\text { IA } * *-0.448), \\
& \mathrm{CU}=0.0315 *(\mathrm{TRN} * * 0.715) *(\mathrm{DA} * * 0.609) *(\mathrm{IA} * * 0.642),
\end{aligned}
$$




$$
\begin{aligned}
& \mathrm{PB}=0.0430 *(\mathrm{TRN} * * 0.852) *(\mathrm{DA} * * 0.857) *(\text { IA } * * 0.999), \text { and } \\
& \mathrm{ZN}=0.0249 *(\mathrm{TRN} * * 0.793) *(\mathrm{DA} * * 0.628) *(\text { IA } * * 1.104),
\end{aligned}
$$

where

COD is load of chemical oxygen demand in storm runoff, in pounds;

DS is load of dissolved solids in storm runoff, in pounds;

SS is load of suspended solids in storm runoff, in pounds;

TN is load of total nitrogen in storm runoff, in pounds;

TKN is load of total Kjeldahl nitrogen in storm runoff, in pounds;

TP is load of total phosphorus in storm runoff, in pounds;

DP is load of dissolved phosphorus in storm runoff, in pounds;

$C U$ is load of total copper in storm runoff, in pounds;

$\mathrm{PB}$ is load of total lead in storm runoff, in pounds;

$\mathrm{ZN}$ is load of total zinc in storm runoff, in pounds;

TRN is total storm rainfall, in inches;

$D A$ is drainage area, in square miles; and

IA is $1+$ impervious area, in percentage of drainage area.

\section{Mean Concentrations of Constituents in Storm Runoff}

Regional regression models were developed by Driver and Tasker (1990) to estimate mean concentrations of selected water-quality constituents in storm runoff for urban watersheds in which annual rainfall is equal to or greater than 40 in. Mean constituent concentrations estimated by these models were compared to mean concentrations measured in storm runoff at stormwater outfalls in Jefferson County (table 3 ). The correspondence between estimated and measured mean concentrations was considered poor. Attempts to adjust the regional models by regression analysis with mean constituent concentrations in storm runoff measured in Jefferson County were not successful.

Attempts were made to estimate mean constituent concentrations in storm runoff in single storms by dividing estimated loads in storm runoff computed with equations $1-10$ by estimated quantity of runoff computed with equation 11. Satisfactory results were not obtained by this procedure, as judged from comparisons of the estimated mean constituent concentrations to those measured at stormwater outfalls in Jefferson County. Therefore, data from the six stormwater outfalls were used to develop models to estimate mean constituent concentrations in storm runoff in ungaged watersheds in the county.

The methods used to develop these mean concentration models for Jefferson County were similar to those used by Driver and Tasker (1990) to develop regional estimation models. Regression models were developed that related the measured storm-runoff mean constituent concentrations to physical and land-use characteristics of the watersheds and climatic characteristics of each storm. For development of these regression models, the best transformation for the response variable was the logarithmic transformation. Bias-correction factors to adjust for underestimation errors during detransformation were computed for each mode1 using a smearing estimate. A smearing estimate is a nonparametric retransformation method based on the average residuals in original units that 
is calculated with the assumption that the pretransformed variables have equal error variances, or that the mean and the variance are independent (Duan, 1983).

The number of mean constituent concentration measurements in storm runoff, the coefficient of determination $\left(R^{2}\right)$, and the standard error of the estimate for each model are listed in table 8 . The proportion of the total variation of the response variable that is explained by the explanatory variables is indicated by the $R^{2}$ value. Therefore, the $R^{2}$ value is used as a summary measure to judge the fit of the regression model to the data. The standard error of estimate of the mean is an estimate of the standard deviation about the regression. The smaller the standard error of estimate, the more precise will be the estimations. The standard error of estimate, in percent, was calculated by use of the following equation:

$$
\mathrm{SE}=100 *((\mathrm{e} * *(\mathrm{MSE} * 5.302)-1) * * 0.5),
$$

where

$\mathrm{SE}$ is the standard error of estimate, in percent; and, MSE is the mean square error, in log (base 10) units.

Table 8. Summary of statistics for regression models of mean constituent concentrations in storm runoff in single storms from urban watersheds of Jefferson County, Kentucky

\begin{tabular}{lccc}
\hline $\begin{array}{c}\text { Constituent } \\
\text { or } \\
\text { property }\end{array}$ & $\begin{array}{c}\text { Number } \\
\text { of } \\
\text { storms }\end{array}$ & $\begin{array}{c}\text { Coefficient } \\
\text { of } \\
\text { determination } \\
\left(\mathrm{R}^{2}\right)\end{array}$ & $\begin{array}{c}\text { Standard } \\
\text { error of } \\
\text { estimate, } \\
\text { in percent }\end{array}$ \\
\hline Chemical oxygen demand & 19 & 0.67 & 62.5 \\
Biochemical oxygen demand & 17 & .47 & 202 \\
Dissolved solids & 20 & .42 & 75.2 \\
Suspended solids & 20 & .37 & 75.5 \\
Nitrogen, total & 18 & .62 & 28.6 \\
Kjeldahl nitrogen, total & 19 & .72 & 34.8 \\
Phosphorus, total & 20 & .40 & 71.6 \\
Phosphorus, dissolved & 14 & .83 & 64.3 \\
Cadmium, total & 20 & .23 & 45.0 \\
Copper, total & 20 & .58 & 30.8 \\
Lead, total & 20 & .25 & 6.7 \\
Zinc, total & 20 & .58 & 50.7 \\
\hline
\end{tabular}

The models for estimation of mean constituent concentrations in storm runoff in Jefferson County were based on 20 measurements or fewer and are therefore statistically tentative. These models generally have small coefficients of determination and large standard errors of estimate (table 8 ). The drainage areas of the Jefferson County watersheds used to develop the 
mean-concentration models ranged from 0.068 to $0.282 \mathrm{mi}^{2}$, and estimates made for watersheds with drainage areas outside of this range may be subject to error. The following models for estimation of mean constituent concentrations in storm runoff in single storms should be used with caution:

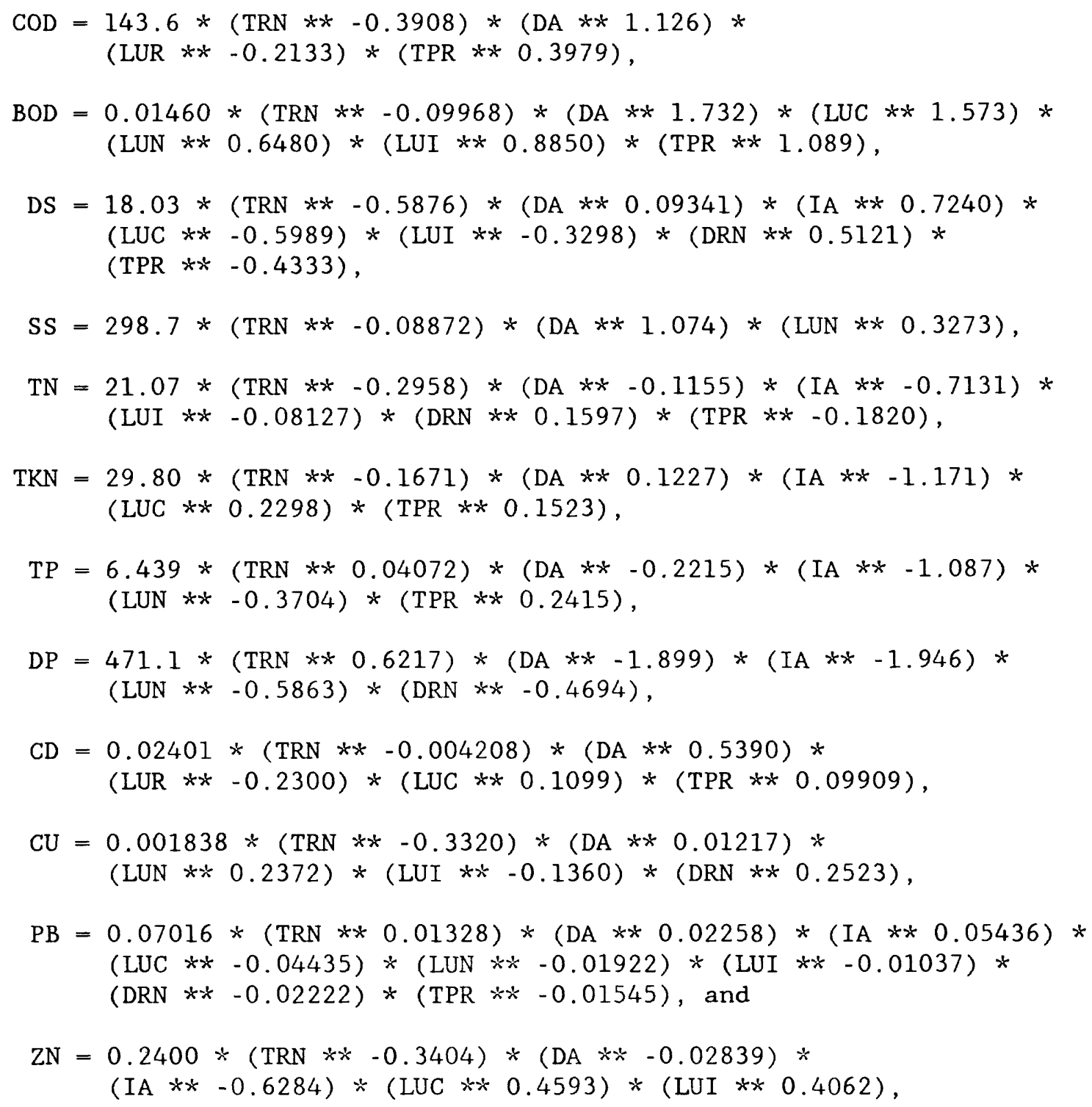

where

COD is mean concentration of chemical oxygen demand in storm runoff, in milligrams per liter;

BOD is mean concentration of biochemical oxygen demand in storm runoff, in milligrams per liter;

DS is mean concentration of dissolved solids in storm runoff, in milligrams per liter;

SS is mean concentration of suspended solids in storm runoff, in milligrams per liter; 
TN is mean concentration of total nitrogen in storm runoff, in milligrams per 1iter;

TKN is mean concentration of total Kjeldah1 nitrogen in storm runoff, in milligrams per liter;

TP is mean concentration of total phosphorus in storm runoff, in milligrams per 1iter;

DP is mean concentration of dissolved phosphorus in storm runoff, in milligrams per liter;

$\mathrm{CD}$ is mean concentration of total cadmium in storm runoff, in milligrams per liter;

$\mathrm{CU}$ is mean concentration of total copper in storm runoff, in micrograms per 1iter;

$\mathrm{PB}$ is mean concentration of total lead in storm runoff, in micrograms per liter;

$\mathrm{ZN}$ is mean concentration of total zinc in storm runoff, in micrograms per 1iter;

TRN is total storm rainfall, in inches;

DA is drainage area, in square miles;

LUC is $1+$ commercial land use, in percentage of drainage area;

LUN is $1+$ nonurban land use, in percentage of drainage area;

LUI is 1 + industrial land use, in percentage of drainage area;

TPR is time since previous rainfall, in hours;

IA is 1 + impervious area, in percentage of drainage area;

DRN is rainfall duration, in minutes; and

LUR is 1 + residential land use, in percentage of drainage area.

\section{Annual Quantity of Storm Runoff}

A computerized method for streamflow partitioning has been developed by the USGS Regional Aquifer System Analysis program (RASA) to estimate groundwater recharge (Rutledge, 1992). Streamflow partitioning is a method for separation of stream discharge records into their base-flow and surface-runoff components. The streamflow-partitioning method developed by the RASA program has two steps: (1) base flow, which includes point-source discharges, is set equal to streamflow during times of negligible runoff, and (2) base flow between these periods (during surface runoff) is interpolated.

An estimate of the daily base-flow component of total discharge was made for each day from January 1, 1988 through December 31, 1992. The surfacerunoff (stormflow) component computed by subtracting the base-flow component from the total discharge. Partitioned hydrographs for four continuous discharge-record and periodic water-quality sampling sites in Jefferson County are shown in figure 5. Before partitioning, total daily discharge was synthesized for all noncontinuous-record sites from relations between measured discharge at the time of sampling and concurrent discharge at a continuous discharge-record site (Evaldi and others, 1993). Summaries of the partitioned streamflow records are presented in the appendix of this report. 


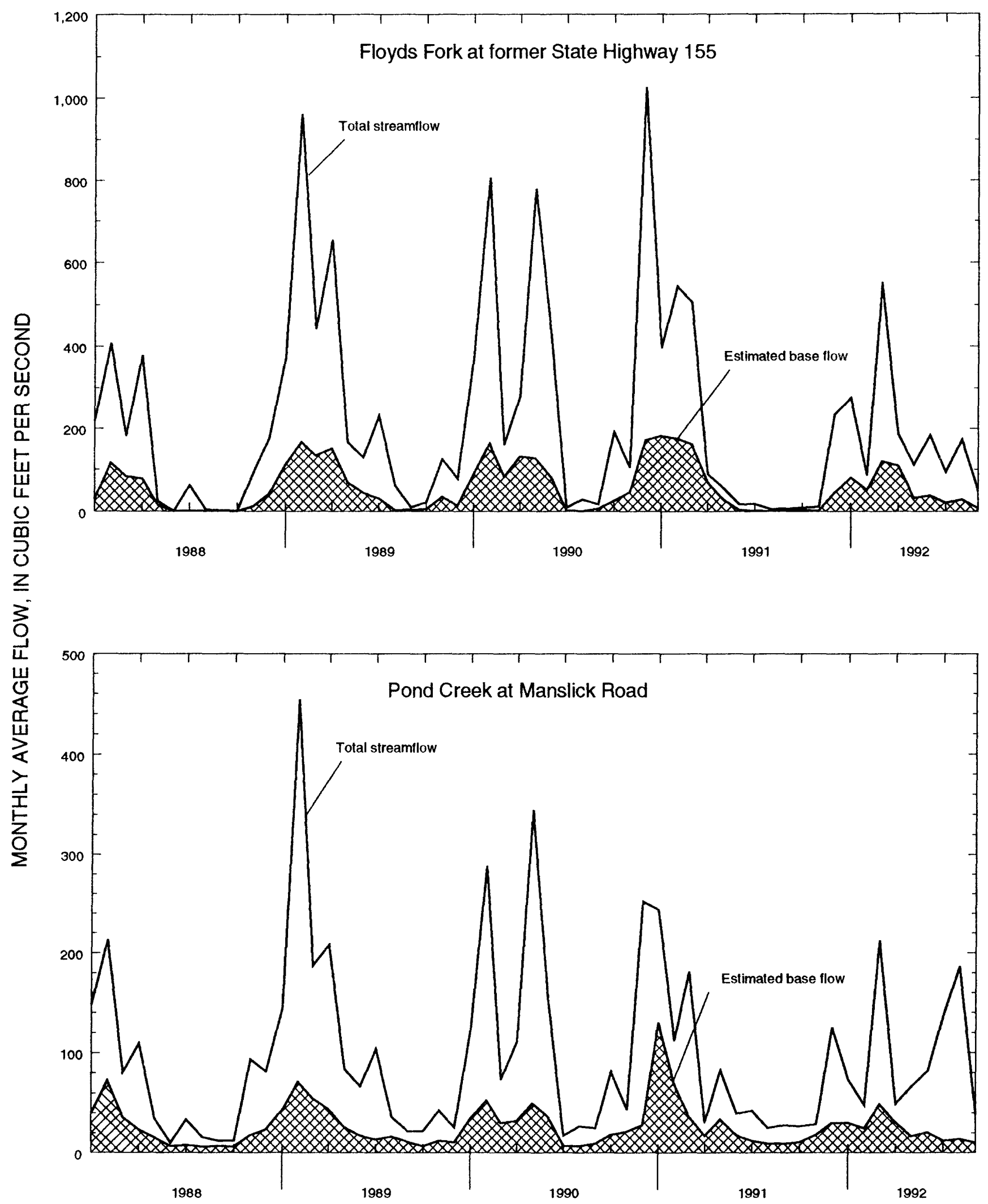

Figure 5. Partitioned flow of selected streams in Jefferson County, Kentucky, 1988-92. 


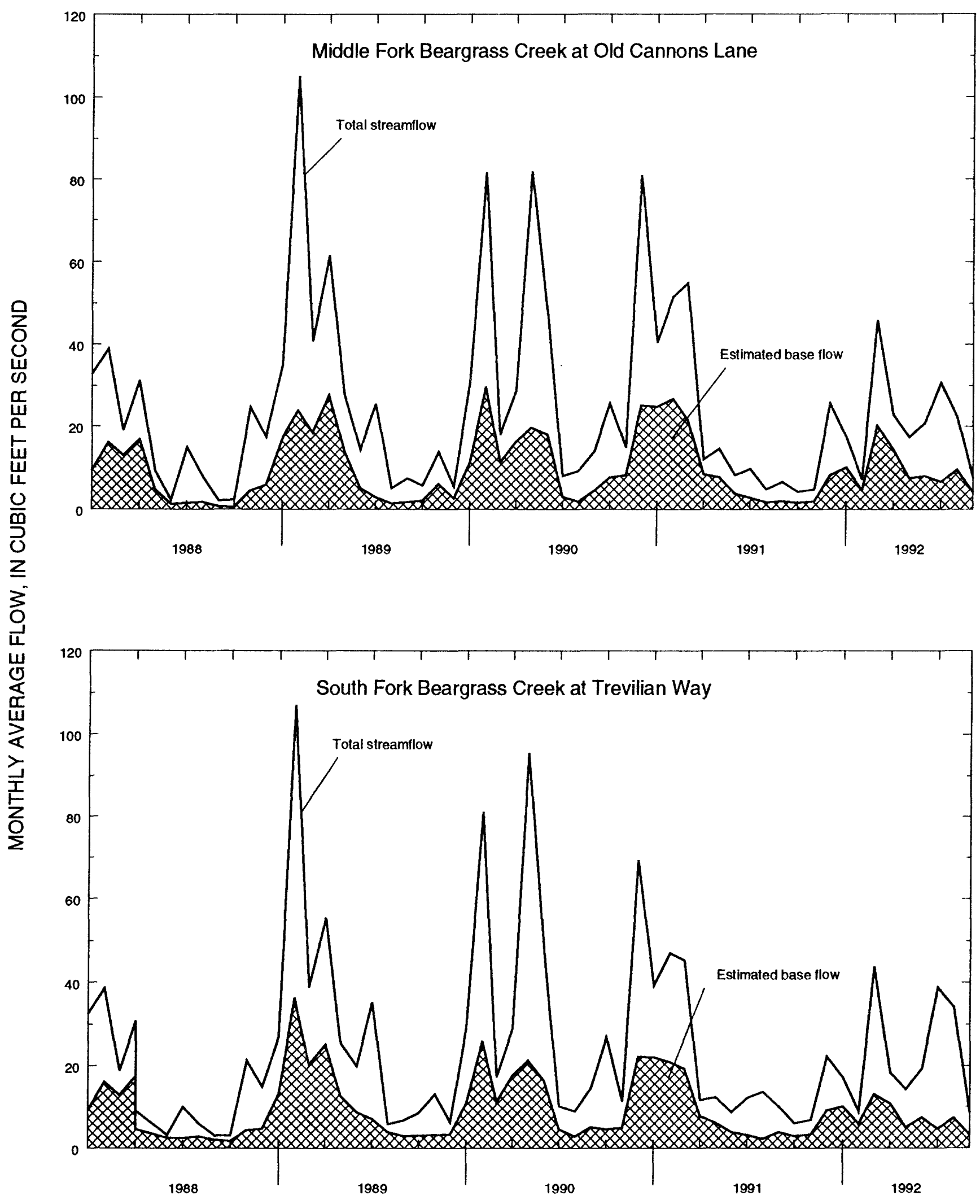

Figure 5. Partitioned flow of selected streams in Jefferson County, Kentucky, 1988-92-Continued. 
A model was developed to estimate annual storm-runoff volumes, on the basis of stormwater runoff calculated for the 25 stream-sampling sites in the county by use of streamflow partitioning. Total annual storm runoff was calculated for each year during 1988-92. In development of the model, the storm-runoff volumes were converted to equivalent rainfall over the drainage basin and were regressed against the annual rainfall, percentage of impervious area, and percentages of land uses in the watersheds. Only annual rainfall, percentage of impervious area, and percentages of commercial and residential land uses were significant in the regression at the 5 -percent level. The regression model for estimating annual storm-runoff volumes accounted for 68 percent of the variation in the dependent variable. In terms of rainfall over the basin, the mean square error of the model was 10.7 in. With conversion factors applied, the model for estimation of annual total stormrunoff volumes from watersheds of Jefferson County is

$$
\begin{aligned}
\text { ROFF }= & ((-9.518+(0.5361 * \text { RAIN })+(0.1798 * \text { IA })-(0.08789 * \text { LUR })- \\
& (0.2930 * \text { LUC })) *(2,323,200 * \text { DA })
\end{aligned}
$$

where

ROFF is annual storm runoff, in cubic feet;

RAIN is total annual rainfall, in inches;

IA is 1 + impervious area, in percentage of drainage area;

LUR is 1 + residential land use, in percentage of drainage area;

LUC is $1+$ commercial land use, in percentage of drainage area; and

DA is drainage area, in square miles.

\section{Annual Loads of Constituents in Storm Runoff}

Annual constituent loads in storm runoff were computed for the 25 urban watersheds of Jefferson County from streamflow records and water-quality samples during 1988-92 and were used to develop models to estimate annual loads in storm runoff from ungaged watersheds in the county. The "dischargerecord method" for annual load estimation, as described by Evaldi and Moore (1992), was used to compute annual loads in base flow and annual total loads. The calibration data used for estimation of annual total loads were based on periodic water-quality samples collected from February 1988 through December 1992. These data are summarized in the appendix of this report. Calibration data used to estimate loads in base flow were subsets of the data used to estimate total loads. Samples were eliminated from the base-flow calibration data set if greater than 10 percent of the streamflow at the time of sampling was from storm runoff.

Total daily streamflow was used for the total-load estimates, and daily estimates of base flow were used to estimate the annual nonstorm loads for each calendar year from 1988 through 1992. Annual base-flow and total-load estimates and statistics describing their accuracies are listed in the appendix of this report. Estimates of annual loads in storm runoff were calculated as the difference between the total annual loads and the total annual loads in base flow. 
Development of models to estimate annual loads in storm runoff was attempted for all water-quality constituents sampled at the 25 stream sites in Jefferson County. Regression models to estimate annual loads of selected constituents in storm runoff for individual years were developed for dissolved oxygen, biochemical and chemical oxygen demands, dissolved and suspended solids, volatile residue, nitrogen, phosphorus and phosphate, calcium, magnesium, barium, and iron. Suitable models to estimate loads in storm runoff could not be developed for nonvolatile suspended solids, copper, and zinc.

The response variables were annual calculations of constituent loads in storm runoff from the 25 stream-sampling sites in Jefferson County and the averages for the period 1988-92. Although one of the assumptions of regression analysis is that the errors are uncorrelated in time, some annual loads may be correlated because they are all based on a continuous series of years (1988-92). Explanatory variables used in the regression analysis included annual rainfall; drainage area; percentage of impervious area; and the percentages of industrial, commercial, residential, and nonurban land use in each basin. Logarithmic transformations of the response and the explanatory variables were done before regression analysis, and a biascorrection factor was calculated for each regression model.

Selection of explanatory variables for each regression model was made by use of stepwise regression procedures available through the Statistical Analysis System ${ }^{2}$ (SAS Institute, 1985). The primary criterion for selecting the most appropriate explanatory variables was that they be significant at the 5 -percent level. Total annual rainfall and total contributing drainage area were the most significant explanatory variables in all the regression models. The number of storm-load calculations used for the response variables, the coefficients of determination ( $\left.\mathrm{R}^{2} \mathrm{~s}\right)$, and standard error of the estimates for each estimation model are listed in table 9. The standard error of estimate, in percent, was calculated by use of equation 22 .

${ }^{2}$ Use of a firm name in this report is for identification purposes only and does not constitute endorsement by the U.S. Geological Survey. 
Table 9. Summary of statistics for regression models of annual loads of selected constituents in storm runoff from urban watersheds of Jefferson County, Kentucky

\begin{tabular}{lccc}
$\begin{array}{c}\text { Constituent } \\
\text { or } \\
\text { property }\end{array}$ & $\begin{array}{c}\text { Number } \\
\text { of } \\
\text { comparisons }\end{array}$ & $\begin{array}{c}\text { Coefficient } \\
\text { of } \\
\text { determination } \\
\text { (R2) }\end{array}$ & $\begin{array}{c}\text { Standard } \\
\text { error of } \\
\text { estimate, } \\
\text { in percent }\end{array}$ \\
\hline Dissolved oxygen & 148 & 0.92 & 41.1 \\
Biochemical oxygen demand & 145 & .83 & 61.3 \\
Chemical oxygen demand & 135 & .83 & 67.2 \\
Dissolved solids & 147 & .92 & 41.7 \\
Suspended solids & 148 & .78 & 101 \\
Residue, volatile nonfilterable & 141 & .62 & 156 \\
Organic nitrogen, dissolved as N & 147 & .80 & 71.5 \\
Ammonia nitrogen, total as N & 118 & .49 & 206 \\
Nitrite nitrogen, total as N & 135 & .66 & 132 \\
Nitrate nitrogen, total as N & 148 & .73 & 78.5 \\
Phosphorus, total as P & 147 & .82 & 60.2 \\
Phosphorus, orthophosphate, total as P 146 & .74 & 78.0 \\
Phosphate, total as PO & 146 & .55 & 146 \\
Calcium, total as Ca 4 & 111 & .90 & 54.9 \\
Magnesium, total as Mg & 111 & .83 & 69.7 \\
Barium, total as Ba & 112 & .71 & 173 \\
Iron, total as Fe & 99 & .83 & 136 \\
\hline
\end{tabular}

The drainage areas of the Jefferson County watersheds used to develop the models for estimation of annual constituent loads in storm runoff ranged from 2.4 to $214 \mathrm{mi}^{2}$, and estimates made for watersheds with drainage areas outside of this range may be subject to error. The regression models that can be used to estimate annual loads of selected constituents in storm runoff from urban watersheds in the county are

$$
\begin{aligned}
\mathrm{DO}= & 14.14 *(\mathrm{AR} * * 1.975) *(\mathrm{DA} * * 1.055) *(\mathrm{LUR} * *-0.1860), \\
\mathrm{BOD}= & 8.887 *(\mathrm{AR} * * 1.938) *(\mathrm{DA} * * 0.9648) * \\
& (\mathrm{LUC} * *-0.2033) *(\mathrm{LUI} * * 0.2231), \\
\mathrm{COD}= & 15.95 *(\mathrm{AR} * * 2.363) *(\mathrm{DA} * * 0.9171) * \\
& (\mathrm{LUR} * *-0.3552) *(\mathrm{LUI} * * 0.2329), \\
\mathrm{DS}= & 2,036 *(\mathrm{AR} * * 1.826) *(\mathrm{DA} * * 1.093) * \\
& (\mathrm{LUC} * *-0.3305) *(\mathrm{LUN} * *-0.3132), \\
\mathrm{SS}= & 0.9707 *(\mathrm{AR} * * 2.935) *(\mathrm{DA} * * 1.252) *(\mathrm{LUI} * * 0.2252), \\
\mathrm{NVR}= & 1.423 *(\mathrm{AR} * * 2.958) *(\mathrm{DA} * * 1.228) *(\mathrm{LUN} * *-0.4227),
\end{aligned}
$$




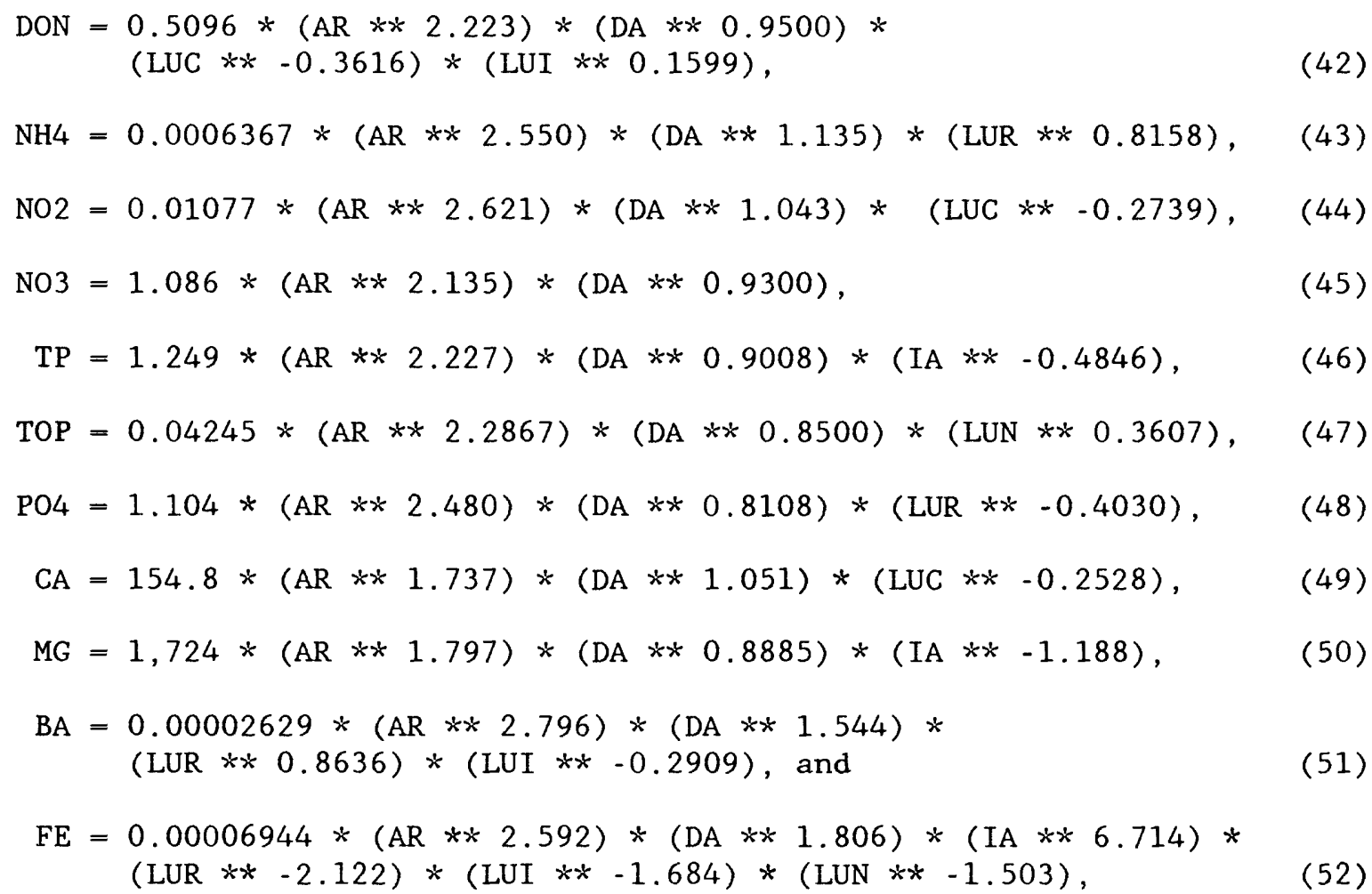

where

DO is annual load of dissolved oxygen in storm runoff, in pounds;

BOD is annual load of biochemical oxygen demand in storm runoff, in pounds;

COD is annual load of chemical oxygen demand in storm runoff, in pounds;

DS is annual load of dissolved solids in storm runoff, in pounds;

SS is annual load of suspended solids in storm runoff, in pounds;

NVR is annual load of nonfilterable volatile residue in storm runoff, in pounds;

DON is annual load of dissolved organic nitrogen in storm runoff, in pounds as $\mathrm{N}$;

NH4 is annual load of total ammonia nitrogen in storm runoff, in pounds as $\mathrm{N}$;

NO2 is annual load of total nitrite nitrogen in storm runoff, in pounds as $\mathrm{N}$;

N03 is annual load of total nitrate nitrogen in storm runoff, in pounds as $\mathrm{N}$;

TP is annual load of total phosphorus in storm runoff, in pounds as P;

TOP is annual load of total orthophosphate in storm runoff, in pounds as $P$;

PO4 is annual load of total phosphate in storm runoff, in pounds as $\mathrm{PO}_{4}$

$\mathrm{CA}$ is annual load of total calcium in storm runoff, in pounds as $\mathrm{Ca}$;

MG is annual load of total magnesium in storm runoff, in pounds as $\mathrm{Mg}$;

$B A$ is annual load of total barium in storm runoff, in pounds as $\mathrm{Ba}$;

$\mathrm{FE}$ is annual load of total iron in storm runoff, in pounds as $\mathrm{Fe}$;

$A R$ is total annual rainfall, in inches; 
DA is drainage area, in square miles;

LUR is 1 + residential land use, in percentage of drainage area;

LUC is 1 + commercial land use, in percentage of drainage area;

LUI is $1+$ industrial land use, in percentage of drainage area;

LUN is $1+$ nonurban land use, in percentage of drainage area, and

IA is $1+$ impervious area, in percentage of drainage area.

\section{Mean Annual Loads of Constituents in Storm Runoff}

Because the mean annual rainfall for 1988 through 1992 was representative of the long term, the averages of the annual loads computed for those years by use of the discharge-record method were considered good estimates of long-term mean annual loads from the 25 urban watersheds of Jefferson County. Annual rainfall totals 1988 to 1992 for Louisville ranged from 37.53 to 57.47 in. and averaged $45.0 \mathrm{in.}$, which is within 5 percent of the long-term (1960-90)

average rainfall of 43.0 in. (National Oceanic and Atmospheric Administration, 1990, 1991-92). The average 1988-92 estimates of constituent loads in storm runoff for the 25 urban watersheds of Jefferson County were used to evaluate the accuracy of regional models for estimation of mean annual loads of selected constituents in storm runoff at ungaged sites in the county.

Driver and Tasker (1990) developed regional regression models to estimate mean loads of selected constituents in single storms on the basis of physical, land-use, and climatic characteristics of the watershed (equations 12-21). Mean annual loads of selected constituents in storm runoff are subsequently estimated in the regional procedures by multiplying the mean constituent loads in single storms by the average number of storms per year; that number is 69 in the Jefferson County area, according to Steurer and Nold (1986). Evaldi and Moore (1992) estimated mean annual loads of selected constituents in storm runoff from urban watersheds of Jefferson County by use of the regional

procedures. The accuracy of these procedures was assessed by comparison of the regional model estimates for the 25 urban watersheds in the county to the 1988-92 mean annual loads of selected constituents in storm runoff estimated on the basis of the discharge-record method.

Plots of average 1988-92 annual loads of constituents in storm runoff for the 25 urban watersheds of Jefferson County against estimates of mean annual loads in storm runoff for the 25 sites based on the regional models showed close linear relations, except for zinc, but indicated that adjustments were needed in the regional estimation models. Adjustments were determined by simple linear regression analysis between the regional model estimates and the 1988-92 discharge-record method estimates, with the regression intercepts set to zero (table 10). 
Table 10. Adjustments to regional regression models for estimating mean annual loads of selected constituents in storm runoff from urban watersheds of Jefferson County, Kentucky

\begin{tabular}{|c|c|c|c|c|}
\hline \multirow[b]{2}{*}{$\begin{array}{c}\text { Constituent } \\
\text { or } \\
\text { property }\end{array}$} & \multirow[b]{2}{*}{$\begin{array}{l}\text { Number } \\
\text { of data } \\
\text { pairs }\end{array}$} & \multirow[b]{2}{*}{$\begin{array}{c}\text { Regression } \\
\text { adjustment } \\
\text { factor }\end{array}$} & \multicolumn{2}{|c|}{ Mean residual, in tons } \\
\hline & & & $\begin{array}{l}\text { Unadjusted } \\
\text { regression } \\
\text { mode1 }\end{array}$ & $\begin{array}{c}\text { Adjusted } \\
\text { regression } \\
\text { mode1 } \\
\end{array}$ \\
\hline Chemical oxygen demand & 23 & 0.82 & -234 & -49.0 \\
\hline Dissolved solids & 25 & 6.02 & 7,550 & -989 \\
\hline Suspended solids & 25 & 2.12 & 2,830 & $-1,150$ \\
\hline Nitrogen, total & 19 & 3.28 & 54.3 & -10.9 \\
\hline Kjeldahl nitrogen, total & 20 & 1.12 & 1.53 & -1.20 \\
\hline Phosphorus, total & 25 & 2.41 & 10.2 & -.206 \\
\hline Copper, total & 12 & 1.37 & .105 & -.144 \\
\hline
\end{tabular}

The drainage areas of the watersheds used to develop the regional models for mean annual loads ranged from 0.014 to $0.830 \mathrm{mi}^{2}$. Watersheds whose drainage areas are greater than $0.5 \mathrm{mi}^{2}$ are calculated as though the drainage areas are $0.2 \mathrm{mi}^{2}$, and loads are then adjusted on the basis of actual drainage area. This method of estimation is based on the assumption that the estimated constituent loads are conservative and cumulative, an assumption that may not always be valid (Tasker and others, 1990). The adjusted regional regression models that can be used to estimate mean annual loads of selected constituents in storm runoff from urban watersheds of Jefferson County are

$$
\begin{aligned}
\mathrm{COD}= & (10 * *(1.1174+(2.0069 * \mathrm{SQRTDA})+(0.0051 * \mathrm{IA}))) * 1.064, \\
\mathrm{DS}= & (10 *(1.8449+(2.5468 * \mathrm{SQRTDA})+(-0.0232 * \mathrm{MJT}))) * 519.6, \\
\mathrm{SS}= & (10 * *(1.5430+(1.5906 * \mathrm{SQRTDA})+(0.0264 * \mathrm{MAR})+ \\
& (-0.0297 * \mathrm{MJT}))) * 222.5, \\
\mathrm{TN}= & (10 * *(-0.2433+(1.6383 * \mathrm{SQRTDA})+(0.0061 * \mathrm{IA})+ \\
& (-0.4442 * \mathrm{X} 2))) * 304.4, \\
\mathrm{TKN}= & (10 * *(-0.7282+(1.6123 * \mathrm{SQRTDA})+(0.0064 * \mathrm{IA})+ \\
& (0.0226 * \mathrm{MAR})+(-0.0210 * \mathrm{MJT})+(-0.4345 * \mathrm{X} 2))) * 98.67, \\
\mathrm{TP}= & (10 * *(-1.3884+(2.0825 * \mathrm{SQRTDA})+(0.0234 * \mathrm{MAR})+ \\
& (-0.0213 * \mathrm{MJT}))) * 218.5, \text { and } \\
\mathrm{CU}= & (10 * *(-1.4824+(1.8281 * \mathrm{SQRTDA})+(-0.0141 * \mathrm{MJT}))) * 132.6, \\
\text { where } &
\end{aligned}
$$

COD is mean annual load of chemical oxygen demand in storm runoff, in pounds; 
DS is mean annual load of dissolved solids in storm runoff, in pounds;

SS is mean annual load of suspended solids in storm runoff, in pounds;

TN is mean annual load of total nitrogen in storm runoff, in pounds;

TKN is mean annual load of total Kjeldahl nitrogen in storm runoff, in pounds;

TP is mean annual load of total phosphorus in storm runoff, in pounds;

$\mathrm{CU}$ is mean annual load of total recoverable copper in storm runoff, in pounds;

SQRTDA is square root of drainage area, in square miles;

IA is impervious area, in percent;

MJT is mean minimum January temperature $\left(26.2^{\circ} \mathrm{F}\right.$; National Oceanic and Atmospheric Administration, 1990);

MAR is mean annual rainfall ( 43.0 in., National Oceanic and Atmospheric Administration, 1990); and

$\mathrm{X} 2$ is 1 if commercial + industrial land use $>75$ percent, or 0 if $<75$ percent.

Estimates of long-term mean annual loads of selected constituents in storm runoff were computed for the urban watersheds monitored at 25 streamsampling sites in Jefferson County. These estimates included (1) mean annual loads of selected constituents in storm runoff for the 1988-92 period calculated with the discharge-record method, (2) mean annual loads in storm runoff based on adjusted regional regression models (equations 53-59), and (3) annual loads in storm runoff computed for an average rainfall year of 43.0 in. by use of models developed from annual-constituent-load and rainfall data in Jefferson County (equations 36-52). Estimates based on the dischargerecord method were considered good and were the basis for adjusting or developing the two sets of estimation equations. Estimates of mean annual loads of selected constituents in storm runoff based on these three computation methods agreed reasonably well (table 11). 
Table 11. Estimates of mean annual loads of selected constituents in storm runoff from urban watersheds of Jefferson County, Kentucky

[DRM, mean annual load in storm runoff estimated by use of the discharge-record method for 1988-92; JC, mean annual load in storm runoff estimated by use of Jefferson County regression equations, with average annual rainfall of 43.03 inches; DT, mean annual load in storm runoff estimated by use of adjusted Driver and Tasker models; Cr, Creek; SF, South Fork; MF, Middle Fork; --, unknown. All loads are in tons]

Pond $\mathrm{Cr}$ at Pendleton Road

$\mathrm{Mill} \mathrm{Cr}$ at Orell Road

Pond $\mathrm{Cr}$ at Manslick Road

SF Beargrass $\mathrm{Cr}$ at Winter Avenue

$\mathrm{SF}$ Beargrass $\mathrm{Cr}$ at Trevilian Way

MF Beargrass $\mathrm{Cr}$ at Old Cannons Lane

MF Beargrass $\mathrm{Cr}$ at Beals Branch Road

Spring Ditch at Private Drive

Muddy Fork at Mockingbird Valley Road

Goose $\mathrm{Cr}$ at U.S. Highway 42

Little Goose $\mathrm{Cr}$ at U.S. Highway 42

Goose $\mathrm{Cr}$ at Old Westport Road

Pope Lick at Pope Lick Road

Floyds Fork at former State Highway 155

Chenoweth Run at Gelhaus Road

Fern $\mathrm{Cr}$ at Old Bardstown Road

Northern Ditch at Preston Highway

Fishpool $\mathrm{Cr}$ at Bost Road

Southern Ditch at Minors Lane

Floyds Fork at Bardstown Road

Cedar $\mathrm{Cr}$ at Thixton Road

Pennsylvania Run at Mt. Washington Road

Mill Cr Cutoff at Dover Road

Harrods $\mathrm{Cr}$ at Hunting $\mathrm{Cr}$ Drive

Long Run at State Highway 1531

Pond $\mathrm{Cr}$ at Pendleton Road

Mill $\mathrm{Cr}$ at Orell Road

Pond $\mathrm{Cr}$ at Manslick Road

$\mathrm{SF}$ Beargrass $\mathrm{Cr}$ at Winter Avenue

SF Beargrass $\mathrm{Cr}$ at Trevilian Way

MF Beargrass $\mathrm{Cr}$ at Old Cannons Lane

MF Beargrass $\mathrm{Cr}$ at Beals Branch Road

Spring Ditch at Private Drive

Muddy Fork at Mockingbird Valley Road

Goose $\mathrm{Cr}$ at U.S. Highway 42

Little Goose $\mathrm{Cr}$ at U.S. Hi ghway 42

Goose $\mathrm{Cr}$ at Old Westport Road

Pope Lick at Pope Lick Road

Floyds Fork at former State Highway 155

Chenoweth Run at Gelhaus Road

Fern $\mathrm{Cr}$ at Old Bardstown Road

Northern Ditch at Preston Highway

Fishpool $\mathrm{Cr}$ at Bost Road

Southern Ditch at Minors Lane

Floyds Fork at Bardstown Road

Cedar $\mathrm{Cr}$ at Thixton Road

Pennsylvania Run at Mt. Washington Road

Mil1 Cr Cutoff at Dover Road

Harrods $\mathrm{Cr}$ at Hunting $\mathrm{Cr}$ Drive

Long Run at State Highway 1531

Pond $\mathrm{Cr}$ at Pendleton Road

$\mathrm{Mill} \mathrm{Cr}$ at Orell Road

Pond $\mathrm{Cr}$ at Manslick Road

SF Beargrass $\mathrm{Cr}$ at Winter Avenue

SF Beargrass $\mathrm{Cr}$ at Trevilian Way

MF Beargrass $\mathrm{Cr}$ at Old Cannons Lane

MF Beargrass $\mathrm{Cr}$ at Beals Branch Road

Spring Ditch at Private Drive

Muddy Fork at Mockingbird Valley Road

Goose $\mathrm{Cr}$ at U.S. Highway 42

Little Goose $\mathrm{Cr}$ at U.S. Highway 42

Goose $\mathrm{Cr}$ at Old Westport Road

Pope Lick at Pope Lick Road

Floyds Fork at former State Highway 155

Chenoweth Run at Gelhaus Road

Fern $\mathrm{Cr}$ at Old Bardstown Road

Northern Ditch at Preston Highway

Fishpool $\mathrm{Cr}$ at Bost Road

Southern Ditch at Minors Lane

Floyds Fork at Bardstown Road

Cedar $\mathrm{Cr}$ at Thixton Road

Pennsylvania Run at Mt. Washington Road

Mil1 Cr Cutoff at Dover Road

Harrods $\mathrm{Cr}$ at Hunting $\mathrm{Cr}$ Drive

Long Run at State Highway 1531
Dissolved oxygen

$\begin{array}{rc}508 & 629 \\ 63.7 & 94.7 \\ 556 & 478 \\ 198 & 149 \\ 115 & 111 \\ 148 & 122 \\ 219 & 151 \\ 17.1 & 16.3 \\ 46.5 & 38.6 \\ 48.8 & 62.9 \\ 43.8 & 41.4 \\ 30.5 & 42.6 \\ 20.8 & 18.5 \\ 1,880 & 1,320 \\ 161 & 81.3 \\ 36.0 & 19.8 \\ 83.8 & 73.2 \\ 54.5 & 33.5 \\ 95.4 & 85.9 \\ 3,020 & 2,080 \\ 85.3 & 81.9 \\ 51.0 & 42.3 \\ 118 & 103 \\ 756 & 1,010 \\ 320 & 235\end{array}$

$\begin{array}{lc}-- & 1,480 \\ -- & 229 \\ -- & 2,230 \\ -- & 468 \\ -- & -- \\ -- & -- \\ -- & 382 \\ -- & 126 \\ -- & 131 \\ -- & 100 \\ -- & 127 \\ -- & 93.2 \\ -- & 59.4 \\ -- & 3,350 \\ -- & 298 \\ -- & 56.8 \\ -- & 251 \\ -- & 129 \\ -- & 293 \\ -- & 5,330 \\ -- & 263 \\ -- & 122 \\ -- & 435 \\ -- & 1,630 \\ -- & 692\end{array}$

1,500

1,230

, 230

434
341

299

94.1

113

161
92.4

101

2,460

460
280

39.8

190
86.8

86.
181

3,750

171

101
272

2,280

648

2, 150

357

1,720

667

504

533
655

77.1

197

248

144

173

3, 180 .

294

86.9

294

136

337

4,910

262

144
452

2, 310

534
Biochemical oxygen demand

$\begin{array}{rc}564 & 466 \\ 53.1 & 81.2 \\ 634 & 372 \\ 263 & 130 \\ 176 & 100 \\ 79.9 & 82.5 \\ 87.9 & 108 \\ 18.0 & 19.4 \\ 24.2 & 37.1 \\ 38.7 & 49.4 \\ 34.2 & 20.7 \\ 24.9 & 30.4 \\ 14.7 & 10.8 \\ 763 & 709 \\ 149 & 86.8 \\ 40.5 & 16.4 \\ 76.5 & 64.4 \\ 31.0 & 30.7 \\ 57.8 & 60.5 \\ 110 & 1,120 \\ 82.4 & 62.2 \\ 38.1 & 36.8 \\ 162 & 88.1 \\ 489 & 530 \\ 197 & 138\end{array}$

Magnesium, total as $\mathrm{Mg}$

$\begin{array}{rr}910 & 696 \\ 30.0 & 157 \\ 1,070 & 510 \\ 245 & 148 \\ 105 & 116 \\ -- & 136 \\ -- & 162\end{array}$

15.217 .3

$36.2 \quad 48.1$

$\begin{array}{rr}69.9 & 120\end{array}$

$58.2 \quad 86.2$

$81.0 \quad 45.9$

1,750

523

83.6

193

--

3,170

262

140

1,260

260
1,970

145

46.2

128

148

2,990

204

123
129

1,460

476
Calcium, total as $\mathrm{Ca}$

$\begin{array}{rr}2,480 & 3,020 \\ 112 & 502 \\ 3,020 & 2,230 \\ 665 & 673 \\ 461 & 505 \\ -- & 508 \\ -- & 637 \\ 61.4 & 66.0 \\ 313 & 199 \\ 263 & 308 \\ 229 & 168 \\ 174 & 206 \\ 180 & 84.4 \\ 8,490 & 7,100 \\ 1,070 & 447 \\ 202 & 140 \\ 406 & 401 \\ -- & 203 \\ -- & 460 \\ 16,400 & 11,400 \\ 520 & 572 \\ 240 & 297 \\ -- & 508 \\ 6,880 & 4,750 \\ -- & 1,180\end{array}$

--
--
--
--
--
--
--
--
--
--
--
--
--
--
--
--
--
--
--
--
--
--
--
--
--

Dissolved solids

$\begin{array}{rrr}16,400 & 16,900 & 25,300 \\ 1,170 & 2,650 & 4,290 \\ 16,700 & 12,800 & 19,700 \\ 6,850 & 4,780 & 6,860 \\ 3,750 & 3,450 & 5,220 \\ 3,770 & 3,240 & 5,680 \\ 5,270 & 4,380 & 6,980 \\ 329 & 382 & 740 \\ 1,940 & 1,580 & 2,000 \\ 1,150 & 1,740 & 3,030 \\ 1,080 & 746 & 1,790 \\ 857 & 1,140 & 2,100 \\ 759 & 418 & 927 \\ 51,300 & 36,700 & 42,600 \\ 6,460 & 2,380 & 3,610 \\ 1,020 & 833 & 1,050 \\ 2,060 & 2,320 & 3,520 \\ 1,450 & 1,110 & 1,670 \\ 2,950 & 2,520 & 4,050 \\ 71,000 & 60,500 & 66,200 \\ 2,760 & 2,910 & 3,490 \\ 1,580 & 1,500 & 1,910 \\ 3,520 & 3,380 & 4,940 \\ 19,700 & 23,500 & 30,900 \\ 7,390 & 5,590 & 7,340\end{array}$


Table 11. Estimates of mean annual loads of selected constituents in storm runoff from urban watersheds of Jefferson County, Kentucky--Continued

[DRM, mean annual load in storm runoff estimated by use of the discharge-record method for 1988-92; JC, mean annual load in storm runoff estimated by use of Jefferson County regression equations, with average annual rainfall of 43.03 inches; DT, mean annual load in storm runoff estimated by use of adjusted Driver and Tasker models; Cr, Creek SF, South Fork; MF, Middle Fork; --, unknown. All loads are in tons]

\begin{tabular}{|c|c|c|c|c|c|c|c|}
\hline \multicolumn{2}{|c|}{ Site number and name } & $\overline{\mathrm{DRM}}$ & $\overline{\mathrm{JC}}$ & DT & DRM & $\mathrm{JC}$ & $\overline{\mathrm{DT}}$ \\
\hline & \multirow{8}{*}{$\begin{array}{l}\text { Pond } \mathrm{Cr} \text { at Pendleton Road } \\
\text { Mill } \mathrm{Cr} \text { at Orell Road } \\
\text { Pond } \mathrm{Cr} \text { at Manslick Road } \\
\text { SF Beargrass } \mathrm{Cr} \text { at Winter Avenue } \\
\text { SF Beargrass } \mathrm{Cr} \text { at Trevilian Way } \\
\text { MF Beargrass } \mathrm{Cr} \text { at Old Cannons Lane } \\
\text { MF Beargrass } \mathrm{Cr} \text { at Beals Branch Road }\end{array}$} & \multicolumn{3}{|c|}{ Suspended solids } & Residue, & \multicolumn{2}{|c|}{ volatile nonfilterable } \\
\hline 1 & & 20,500 & 12,400 & 18,700 & 3,100 & 2,170 & -- \\
\hline 2 & & 1,220 & 1,230 & 3,160 & 264 & 243 & -- \\
\hline 3 & & 21,300 & 9,540 & 14,500 & 4,460 & 1,700 & -- \\
\hline 5 & & 7,250 & 2,650 & 5,090 & 3,080 & 686 & -- \\
\hline 6 & & 4,830 & 1,880 & 3,860 & 1,710 & 469 & -- \\
\hline 7 & & 773 & 1,700 & 4,200 & 449 & 488 & -- \\
\hline 8 & & 1,090 & 2,340 & 5,150 & 267 & 684 & -- \\
\hline 9 & Spring Ditch at Private Drive & 177 & 206 & 547 & 38.2 & 37.8 & -- \\
\hline 10 & Muddy Fork at Mockingbird Valley Road & 490 & 499 & 1,480 & 97.5 & 183 & -- \\
\hline 11 & Goose $\mathrm{Cr}$ at U.S. Highway 42 & 455 & 743 & 2,230 & 96.9 & 185 & -- \\
\hline 12 & Little Goose $\mathrm{Cr}$ at U.S. Highway 42 & 542 & 279 & 1,320 & 106 & 73.9 & -- \\
\hline 13 & Goose $\mathrm{Cr}$ at Old Westport Road & 529 & 417 & 1,550 & 146 & 118 & -- \\
\hline 14 & Pope Lick at Pope Lick Road & 342 & 120 & 683 & 49.8 & 39.6 & -- \\
\hline 15 & Floyds Fork at former State Highway 155 & 19,500 & 17,100 & 31,400 & 5,730 & 3,140 & -- \\
\hline 16 & Chenoweth Run at Gelhaus Road & 2,490 & 1,190 & 2,670 & 707 & 195 & -- \\
\hline 17 & Fern $\mathrm{Cr}$ at Old Bardstown Road & 520 & 140 & 774 & 192 & 50.1 & -- \\
\hline 18 & Northern Ditch at Preston Highway & 1,350 & 931 & 2,590 & 190 & 219 & -- \\
\hline 19 & Fishpool Cr at Bost Road & 461 & 329 & 1,230 & 126 & 81.0 & -- \\
\hline 20 & Southern Ditch at Minors Lane & 1,060 & 915 & 2,990 & 299 & 240 & -- \\
\hline 21 & Floyds Fork at Bardstown Road & 62,700 & 30,500 & $4 \overline{8}, 800$ & 12,900 & 5,470 & -- \\
\hline 22 & Cedar $\mathrm{Cr}$ at Thixton Road & 408 & 669 & 2,570 & 98.4 & 157 & -- \\
\hline 23 & Pennsylvania Run at Mt. Washington Road & 148 & 339 & 1,410 & 37.5 & 77.5 & -- \\
\hline 24 & Mill Cr Cutoff at Dover Road & 1,420 & 1,550 & 3,650 & 286 & 413 & -- \\
\hline 25 & Harrods $\mathrm{Cr}$ at Hunting $\mathrm{Cr}$ Drive & 6,660 & 12,300 & 22,700 & -- & 2,090 & -- \\
\hline 26 & Long Run at State Highway 1531 & 3,650 & 1,920 & 5,410 & 382 & 348 & -- \\
\hline
\end{tabular}

Pond $\mathrm{Cr}_{\mathrm{r}}$ at Pendleton Road

$\mathrm{Mill} \mathrm{Cr}$ at Orell Road

Pond $\mathrm{Cr}$ at Manslick Road

SF Beargrass $\mathrm{Cr}$ at Winter Avenue

SF Beargrass $\mathrm{Cr}$ at Trevilian Way

MF Beargrass $\mathrm{Cr}$ at Old Cannons Lane

MF Beargrass $\mathrm{Cr}$ at Beals Branch Road

Spring Ditch at Private Drive

Muddy Fork at Mockingbird Valley Road

Goose $\mathrm{Cr}$ at U.S. Highway 42

Little Goose $\mathbf{C r}$ at U.S. Highway 42

Goose $\mathrm{Cr}$ at Old Westport Road

Pope Lick at Pope Lick Road

Floyds Fork at former State Highway 155

Chenoweth Run at Gelhaus Road

Fern $\mathrm{Cr}$ at Old Bardstown Road

Northern Ditch at Preston Highway

Fishpool $\mathrm{Cr}$ at Bost Road

Southern Ditch at Minors Lane

Floyds Fork at Bardstown Road

Cedar $\mathrm{Cr}$ at Thixton Road

Pennsylvania Run at Mt. Washington Road

Mill Cr Cutoff at Dover Road

Harrods $\mathrm{Cr}$ at Hunting $\mathrm{Cr}$ Drive

Long Run at State Highway 1531

Pond $\mathrm{Cr}$ at Pendleton Road

$\mathrm{Mill} \mathrm{Cr}$ at Orell Road

Pond $\mathrm{Cr}$ at Manslick Road

SF Beargrass $\mathrm{Cr}$ at Winter Avenue

SF Beargrass $\mathrm{Cr}$ at Trevilian Way

MF Beargrass $\mathrm{Cr}$ at Old Cannons Lane

MF Beargrass $\mathrm{Cr}_{r}$ at Beals Branch Road

Spring Ditch at Private Drive

Muddy Fork at Mockingbird Valley Road

Goose $\mathrm{Cr}$ at U.S. Highway 42

Little Goose $\mathrm{Cr}$ at U.S. Highway 42

Goose $\mathrm{Cr}$ at Old Westport Road

Pope Lick at Pope Lick Road

Floyds Fork at former State Highway 155

Chenoweth Run at Gelhaus Road

Fern $\mathrm{Cr}$ at Old Bardstown Road

Northern Ditch at Preston Highway

Fishpool $\mathrm{Cr}$ at Bost Road

Southern Ditch at Minors Lane

Floyds Fork at Bardstown Road

Cedar $\mathrm{Cr}$ at Thixton Road

Pennsylvania Run at Mt. Washington Road

Mill Cr Cutoff at Dover Road

Harrods $\mathrm{Cr}$ at Hunting $\mathrm{Cr}$ Drive

Long Run at State Highway 1531

Nitrate nitrogen, total as $\mathrm{N}$

134

134
9.34
127
31.4
24.7
24.7
32.5
4.18
9.62
13.1
14.2
9.32
5.49
261
18.5
12.2
20.3
12.0
25.0
484
15.5
7.01
38.1
145
43.8

101

19.3
79.8

30.0

23.2

25.1

30.3

3.7

9.5
13.9

8.5

9.9

4.6

163

16.4

16.1

16.1
8.0

18.3

245

15.9

9.1
22.0

121

31.7

Ammonia nitrogen, total as $\mathrm{N}$

$\begin{array}{rr}18.9 & 13.9 \\ .58 & 2.0 \\ 33.0 & 11.0 \\ 10.7 & 4.3 \\ 3.19 & 3.0 \\ 1.95 & 3.3 \\ 1.27 & 4.3 \\ .34 & .1 \\ .54 & 1.3 \\ .84 & 1.6 \\ .28 & .5 \\ -- & 1.1 \\ .64 & .3 \\ -- & 10.9 \\ -- & 1.4 \\ 1.64 & .5 \\ 1.44 & 2.0 \\ 1.38 & .8 \\ 4.24 & 2.2 \\ 16.5 & 18.4 \\ 1.74 & 1.1 \\ 2.44 & .6 \\ 11.4 & 3.1 \\ -- & 5.4 \\ -- & .8\end{array}$

Nitrite nitrogen, total as $\mathrm{N}$

$\begin{array}{rrr}9.27 & 5.36 & -- \\ -- & .91 & -- \\ 12.9 & 3.96 & -- \\ 1.29 & 1.19 & -- \\ 1.22 & .90 & -- \\ .37 & .90 & -- \\ .48 & 1.12 & -- \\ .14 & .12 & -- \\ .30 & .36 & -- \\ .76 & .55 & -- \\ .38 & .30 & -- \\ .42 & .37 & -- \\ .22 & .15 & -- \\ 12.1 & 12.9 & -- \\ 1.18 & .82 & -- \\ .60 & .26 & -- \\ .67 & .73 & -- \\ .80 & .37 & -- \\ 1.32 & .83 & -- \\ 25.5 & 20.6 & - \\ .72 & 1.07 & -- \\ .76 & .56 & -- \\ 2.26 & .91 & -- \\ 5.70 & 8.59 & -- \\ -- & 2.18 & -\end{array}$

Organic nitrogen, total as N

$\begin{array}{ccc}34.6 & 43.7 & -- \\ 4.11 & 8.40 & -- \\ 37.7 & 33.7 & - \\ 25.2 & 11.2 & - \\ 11.4 & 8.65 & -- \\ 6.03 & 7.17 & -- \\ 6.96 & 9.27 & -- \\ 2.86 & 1.63 & - \\ 1.72 & 3.53 & -- \\ 3.15 & 4.85 & -- \\ 1.84 & 2.17 & - \\ 3.72 & 3.06 & -- \\ 2.54 & 1.15 & -- \\ 160 & 88.0 & -- \\ 8.75 & 8.92 & -- \\ 2.88 & 2.22 & - \\ 11.2 & 6.68 & -- \\ 4.32 & 3.53 & -- \\ 9.88 & 6.56 & -- \\ 167 & 138 & - \\ 7.48 & 8.86 & - \\ 5.70 & 5.10 & -- \\ 24.8 & 8.23 & -- \\ 56.4 & 62.2 & -- \\ 31.3 & 18.1 & -- \\ & & \end{array}$


Table 11. Estimates of mean annual loads of selected constituents in storm runoff from urban watersheds of Jefferson County, Kentucky--Continued

[DRM, mean annual load in storm runoff estimated by use of the discharge-record method for 1988-92; JC, mean annual load in storm rumoff estimated by use of Jefferson County regression equations, with average annual rainfall of 43.03 inches; DT, mean annual load in storm runoff estimated by use of adjusted Driver and Tasker models; Cr, Creek; SF, South Fork; MF, Middle Fork; --, unknown. All loads are in tons]

\begin{tabular}{|c|c|c|c|c|c|c|c|}
\hline & ite number and name & $\mathrm{DRM}$ & $\mathrm{JC}$ & DT & DRM & $\overline{\mathrm{JC}}$ & DT \\
\hline & & Kjeldahl & cogen, & as $\mathrm{N}$ & $\underline{\text { Nit }}$ & 1. tota & \\
\hline 1 & Pond $\mathrm{Cr}$ at Pendleton Road & 53.5 & 57.6 & 79.0 & 196 & 164 & 283 \\
\hline 2 & Mill $\mathrm{Cr}$ at Orell Road & 4.70 & 10.4 & 13.0 & - & 30.7 & 46.6 \\
\hline 3 & Pond $\mathrm{Cr}$ at Manslick Road & 70.7 & 44.7 & 63.5 & 211 & 128 & 228 \\
\hline 5 & SF Beargrass $\mathrm{Cr}$ at Winter Avenue & 35.9 & 15.5 & 25.3 & 68.7 & 46.6 & 90.2 \\
\hline 6 & SF Beargrass $\mathrm{Cr}$ at Trevilian Way & 14.6 & 11.7 & 19.2 & 40.5 & 35.8 & 67.9 \\
\hline 7 & MF Beargrass $\mathrm{Cr}$ at Old Cannons Lane & 7.99 & 10.5 & 20.0 & 33.0 & 36.4 & 71.5 \\
\hline 8 & MF Beargrass $\mathrm{Cr}$ at Beals Branch Road & 8.23 & 13.6 & 24.6 & 41.2 & 45.1 & 87.9 \\
\hline 9 & Spring Ditch at Private Drive & 3.19 & 1.8 & 2.98 & 7.51 & 5.71 & 10.6 \\
\hline 10 & Muddy Fork at Mockingbird Valley Road & 2.26 & 4.8 & 7.47 & 12.2 & 14.7 & 26.6 \\
\hline 11 & Goose $\mathrm{Cr}$ at U.S. Highway 42 & 3.99 & 6.4 & 9.04 & 17.9 & 21.0 & 32.5 \\
\hline 12 & Little Goose $\mathrm{Cr}$ at U.S. Hi ghway 42 & 2.12 & 2.6 & 5.20 & 16.7 & 11.5 & 18.7 \\
\hline 13 & Goose $\mathrm{Cr}$ at old Westport Road & - & 4.1 & 6.28 & - & 14.5 & 22.6 \\
\hline 14 & Pope Lick at Pope Lick Road & 3.18 & 1.5 & 2.69 & 8.89 & 6.32 & 9.68 \\
\hline 15 & Floyds Fork at former State Highway 155 & -- & 98.9 & 113 & - & 275 & 410 \\
\hline 16 & Chenoweth Run at Gelhaus Road & -- & 10.4 & 10.7 & -- & 27.6 & 38.4 \\
\hline 17 & Fern $\mathrm{Cr}$ at Old Bardstown Road & 4.52 & 2.8 & 3.15 & 17.3 & 8.27 & 11.3 \\
\hline 18 & Northern Ditch at Preston Highway & 12.7 & 8.6 & 10.8 & 33.6 & 25.5 & 38.7 \\
\hline 19 & Fishpool $\mathrm{Cr}$ at Bost Road & 5.70 & 4.3 & 4.93 & 18.5 & 12.8 & 17.7 \\
\hline 20 & Southern Ditch at Minors Lane & 14.1 & 8.7 & 12.3 & 40.5 & 27.9 & 44.3 \\
\hline 21 & Floyds Fork at Bardstown Road & 183 & 156 & 174 & 692 & 422 & 630 \\
\hline 22 & Cedar $\mathrm{Cr}$ at Thixton Road & 9.22 & 10.0 & 9.34 & 25.4 & 27.0 & 33.8 \\
\hline 23 & Pennsylvania Run at Mt. Washington Road & 8.15 & 5.7 & 5.10 & 15.9 & 15.4 & 18.4 \\
\hline 24 & Mill Cr Cutoff at Dover Road & 36.2 & 11.4 & 16.9 & 76.5 & 34.3 & 60.4 \\
\hline 25 & Harrods $\mathrm{Cr}$ at Hunting $\mathrm{Cr}$ Drive & -- & 67.6 & 82.1 & - & 197 & 297 \\
\hline 26 & Long Run at State Highway 1531 & -- & 18.9 & 18.8 & -- & 52.8 & 68.2 \\
\hline & & Phosp & total & & $\underline{\mathrm{Phc}}$ & 15, to & \\
\hline 1 & Pond $\mathrm{Cr}$ at Pendleton Road & 75.4 & 50.5 & - & 35.8 & 28.4 & 43.9 \\
\hline 2 & Mill Cr at Orell Road & 9.56 & 11.4 & - & 5.41 & 5.94 & 7.45 \\
\hline 3 & Pond $\mathrm{Cr}$ at Manslick Road & 92.0 & 40.3 & -- & 38.7 & 21.9 & 34.2 \\
\hline 5 & SF Beargrass $\mathrm{Cr}$ at Winter Avenue & 20.0 & 15.1 & -- & 15.0 & 7.48 & 12.0 \\
\hline 6 & SF Beargrass $\mathrm{Cr}$ at Trevilian Way & 17.4 & 12.2 & -- & 7.64 & 5.84 & 9.06 \\
\hline 7 & MF Beargrass $\mathrm{Cr}$ at Old Cannons Lane & 10.5 & 13.2 & -- & 5.69 & 6.51 & 9.86 \\
\hline 8 & MF Beargrass $\mathrm{Cr}$ at Beals Branch Road & 6.94 & 15.2 & -- & 4.81 & 7.83 & 12.1 \\
\hline 9 & Spring Ditch at Private Drive & 2.42 & 3.3 & -- & 1.04 & .94 & 1.29 \\
\hline 10 & Muddy Fork at Mockingbird Valley Road & 2.30 & 5.0 & -- & 1.62 & 2.43 & 3.49 \\
\hline 11 & Goose $\mathrm{Cr}$ at U.S. Highway 42 & 6.72 & 7.8 & -- & 3.96 & 4.41 & 5.25 \\
\hline 12 & Little Goose $\mathrm{Cr}$ at U.S. Highway 42 & 6.73 & 6.8 & -- & 2.84 & 2.86 & 3.11 \\
\hline 13 & Goose $\mathrm{Cr}$ at Old Westport Road & 6.94 & 5.8 & -- & 3.56 & 3.17 & 3.64 \\
\hline 14 & Pope Lick at Pope Lick Road & 3.54 & 3.1 & -- & 2.40 & 1.57 & 1.61 \\
\hline 15 & Floyds Fork at former State Highway 155 & 146 & 116 & -- & 87.2 & 57.5 & 74.0 \\
\hline 16 & Chenoweth Run at Gelhaus Road & 29.5 & 10.6 & -. & 7.78 & 5.24 & 6.27 \\
\hline 17 & Fern $\mathrm{Cr}$ at Old Bardstown Road & 6.49 & 3.0 & -- & 3.64 & 1.69 & 1.82 \\
\hline 18 & Northern Ditch at Preston Highway & 14.1 & 8.7 & -- & 6.63 & 4.91 & 6.10 \\
\hline 19 & Fishpool $\mathrm{Cr}$ at Bost Road & 6.80 & 4.8 & -- & 3.40 & 2.61 & 2.89 \\
\hline 20 & Southerm Ditch at Minors Lane & 12.9 & 10.1 & -- & 6.80 & 5.61 & 7.01 \\
\hline 21 & Floyds Fork at Bardstown Road & 200 & 164 & -- & 116 & 86.3 & 115 \\
\hline 22 & Cedar $\mathrm{Cr}$ at Thixton Road & 9.66 & 11.3 & -- & 3.93 & 5.91 & 6.05 \\
\hline 23 & Pennsylvania Run at Mt. Washington Road & 3.82 & 6.5 & -- & 1.66 & 3.48 & 3.33 \\
\hline 24 & Mi 11 Cr Cutoff at Dover Road & 44.6 & 11.1 & -- & 13.9 & 5.91 & 8.58 \\
\hline 25 & Harrods $\mathrm{Cr}$ at Hunting $\mathrm{Cr}$ Drive & 68.1 & 106 & -- & 36.0 & 42.8 & 53.5 \\
\hline 26 & Long Run at State Highway 1531 & 70.4 & 37.4 & -- & 23.5 & 12.5 & 12.7 \\
\hline
\end{tabular}

Pond $\mathrm{Cr}$ at Pendleton Road

Mill $\mathrm{Cr}$ at Orell Road

Pond $\mathrm{Cr}$ at Manslick Road

$\mathrm{SF}$ Beargrass $\mathrm{Cr}$ at Winter Avenue

SF Beargrass $\mathrm{Cr}$ at Trevilian Way

MF Beargrass $\mathrm{Cr}$ at Old Cannons Lane

MF Beargrass $\mathrm{Cr}$ at Beals Branch Road

Spring Ditch at Private Drive

Muddy Fork at Mockingbird Valley Road

Goose $\mathrm{Cr}$ at U.S. Highway 42

Little Goose $\mathrm{Cr}$ at U.S. Highway 42

Goose $\mathrm{Cr}$ at Old Westport Road

Pope Lick at Pope Lick Road

Floyds Fork at former State Highway 155

Chenoweth Run at Gelhaus Road

Fern $\mathrm{Cr}$ at Old Bardstown Road

Northern Ditch at Preston Highway

Fishpool $\mathrm{Cr}$ at Bost Road

Southern Ditch at Minors Lane

Floyds Fork at Bardstown Road

Cedar $\mathrm{Cr}$ at Thixton Road

Pennsylvania Run at Mt. Washington Road

Mill Cr Cutoff at Dover Road

Harrods $\mathrm{Cr}$ at Hunting $\mathrm{Cr}$ Drive

Long Run at State Highway 1531
Phosphorus, ortophosphate, total as $\mathrm{P}$

24.6
3.38
30.0
6.49
5.85
3.31
2.19
.78
.74
2.20
2.20
2.26
1.16
47.6
9.62
2.12
4.16
2.32
4.51
65.1
3.14
1.24
14.6
22.2
21.3

Barium, total as $\mathrm{Ba}$

3.03

.28

1.43

1.43
.74

--

.06

.28
.18

.18

.12

.08
6.62

.73

.46
.56

$-$.

15.0

.50

.21

4.60

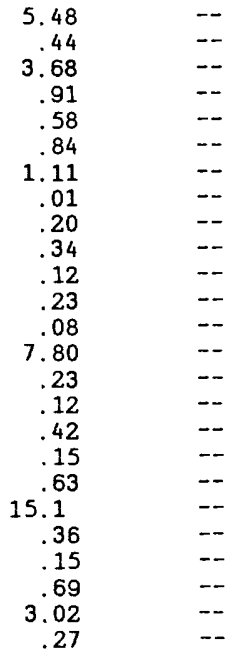


Table 11. Estimates of mean annual loads of selected constituents in storm runoff from urban watersheds of Jefferson County, Kentucky--Continued

[DRM, mean annual load in storm runoff estimated by use of the discharge-record method for 1988-92; JC, mean annual load in storm runoff estimated by use of Jefferson County regression equations, with average annual rainfall of

43.03 inches; DT, mean annual load in storm runoff estimated by use of adjusted Driver and Tasker models; Cr, Creek; SF, South Fork; MF, Middle Fork; --, unknown. All loads are in tons]

\begin{tabular}{|c|c|c|c|c|c|c|c|}
\hline & ite number and name & DRM & $\mathrm{JC}$ & DT & DRM & $\mathrm{JC}$ & $\overline{\mathrm{DT}}$ \\
\hline & & Cor & ota. & & & total as & \\
\hline 1 & Pond $\mathrm{Cr}$ at Pendleton Road & 1.48 & -- & 2.51 & -- & 322 & -- \\
\hline 2 & Mill $\mathrm{Cr}$ at Orell Road & -1 & -- & .43 & 74.4 & 12.2 & -- \\
\hline 3 & Pond $\mathrm{Cr}$ at Manslick Road & 1.60 & -- & 1.96 & $197^{\circ}$ & 262 & -- \\
\hline 5 & SF Beargrass $\mathrm{Cr}$ at Winter Avenue & $\begin{array}{r}1.00 \\
.56\end{array}$ & -- & $\begin{array}{r}1.68 \\
.68\end{array}$ & 850 & 330 & -- \\
\hline 6 & SF Beargrass $\mathrm{Cr}$ at Trevilian Way & -- & -- & .52 & 33.1 & 170 & -- \\
\hline 7 & MF Beargrass $\mathrm{Cr}$ at Old Cannons Lane & -- & -- & .56 & - & 513 & -- \\
\hline 8 & MF Beargrass Cr at Beals Branch Road & -- & -- & .69 & -- & 567 & -- \\
\hline 9 & Spring Ditch at Private Drive & .04 & -- & .07 & 8.98 & 7.52 & -- \\
\hline 10 & Muddy Fork at Mockingbird Valley Road & -- & -- & 20 & 280 & 125 & -- \\
\hline 11 & Goose $\mathrm{Cr}$ at U.S. Highway 42 & .08 & - & .30 & 4.71 & 9.01 & -- \\
\hline 12 & Little Goose $\mathrm{Cr}$ at U.S. Highway 42 & -- & -- & .18 & 22.4 & 39.8 & -- \\
\hline 13 & Goose $\mathrm{Cr}$ at old Westport Road & .06 & -- & .21 & 3.24 & 11.3 & -- \\
\hline 14 & Pope Lick at Pope Lick Road & .02 & -- & .09 & -- & 7.95 & -- \\
\hline 15 & Floyds Fork at former State Highway 155 & 5.11 & -- & 4.23 & 2,840 & 1,260 & -- \\
\hline 16 & Chenoweth Run at Gelhaus Road & - & -- & .36 & 2.68 & $\begin{array}{r}2.07 \\
2.007\end{array}$ & - \\
\hline 17 & Ferm $\mathrm{Cr}$ at Old Bardstown Road & .10 & -- & .10 & 71.7 & 12.0 & -- \\
\hline 18 & Northern Ditch at Preston Highway & .07 & -- & .35 & 36.4 & 11.8 & -- \\
\hline 19 & Fishpool $\mathrm{Cr}$ at Bost Road & - & -- & .17 & - & 2.99 & -- \\
\hline 20 & Southern Ditch at Minors Lane & -- & -- & .40 & -- & 49.5 & -- \\
\hline 21 & Floyds Fork at Bardstown Road & - & -- & 6.56 & 1,580 & 1,890 & $-\infty$ \\
\hline 22 & Cedar $\mathrm{Cr}$ at Thixton Road & .14 & -- & .35 & 4.72 & 11.0 & -- \\
\hline 23 & Pennsylvania Run at Mt. Washington Road & .08 & -- & 19 & $1.4 \overline{0}$ & 1.52 & -- \\
\hline 24 & Mill Cr Cutoff at Dover Road & -- & -- & .49 & $\therefore$ & 108 & -- \\
\hline 25 & Harrods $\mathrm{Cr}$ at Hunting $\mathrm{Cr}$ Drive & -- & -- & 3.07 & 1,800 & 1,010 & -- \\
\hline 26 & Long Run at State Highway 1531 & -- & -- & .73 & - & 83.1 & -- \\
\hline
\end{tabular}


This report presents techniques for estimation of storm-runoff volumes and concentrations and loads of selected constituents in storm runoff from urban watersheds of Jefferson County, Ky. Previously developed regional regression models were evaluated to assess their suitability for use in the county. Comparisons of the regional regression model estimates to data obtained in Jefferson County indicated that, in general, the regional models did not adequately estimate constituent concentrations and loads in the county. Regional regression models were adjusted or other estimation models were developed on the basis of runoff volumes, concentrations, and loads measured in Jefferson County. Data on storm-runoff volumes and concentrations of constituents in storm runoff were collected at six stormwater outfalls in the county from January 1991 through May 1992. Samples were collected for at least three storms at each outfall site. Sampling events were at least 1 month apart and generally were preceeded by a dry period of at least 72 hours. Annual constituent loads in storm runoff were measured at a network of stream sites that were sampled from February 1988 through March 1991.

Adjustments were made to two sets of regional regression models that estimate loads in storm runoff of chemical oxygen demand; dissolved and suspended solids; total nitrogen and total Kjeldahl nitrogen; total and dissolved phosphorus; and total recoverable copper, lead, and zinc. One set of these models requires total storm rainfall, drainage area, impervious area, land-use information, mean annual rainfall, mean annual nitrogen load in precipitation, and mean minimum January temperature as explanatory variables. The other set of models consists of simplified versions that only require total storm rainfall, drainage area, and impervious area as explanatory variables. A regional regression model that requires total storm rainfall, drainage area, and impervious area to estimate total runoff of a storm needed only slight adjustment for estimation in Jefferson County.

The correspondence of storm-runoff mean concentrations measured in Jefferson County to those estimated by regional models was considered poor, and suitable adjustments could not be defined. Regression models based on the data from the six stormwater outfalls were developed to estimate mean concentrations of chemical oxygen demand, dissolved and suspended solids, nitrogen, phosphorus, copper, lead, and zinc in storm runoff. Explanatory variables needed for these models include total storm rainfall and its duration, drainage area, impervious area, and land-use information. The concentration models generally have small coefficients of determination and large standard errors.

Regression models to estimate annual loads in storm runoff for individual years were developed for dissolved oxygen, biochemical and chemical oxygen demands, dissolved and suspended solids, volatile residue, nitrogen, phosphorus and phosphate, calcium, magnesium, barium, and iron. These models were based on loads in storm runoff from watersheds of Jefferson County calculated for 1988 through 1992 by use of the discharge-record method applied to partitioned streamflow records. Explanatory variables required by the models to estimate annual loads in storm runoff include total annual rainfall, drainage area, impervious area, and land use information. 
Average rainfall for 1988-92 was within 5 percent of the long-term average rainfall for the Jefferson County area; therefore, average loads of selected constituents in storm runoff for this period were considered to be good estimates of the long-term mean annual loads for 25 watersheds of Jefferson County. The watershed data were used to adjust regional models for estimation of mean annual loads in storm runoff of chemical oxygen demand, dissolved and suspended solids, total nitrogen and total Kjeldahl nitrogen, total phosphorus, and total recoverable copper. Explanatory variables used in these models include drainage area, impervious area, commercial and industrial land-use information, mean annual rainfall, and minimum January temperature. In addition, annual loads were computed for an average rainfall year of 43.0 in. by use of models developed from annual-constituent-load and rainfall data in Jefferson County. The estimates of mean annual constituent loads in storm runoff based on these three computation methods agreed reasonably well. 
Driver, N.E., and Tasker, G.D., 1990, Techniques for estimation of stormrunoff loads, volumes, and selected constituent concentrations in urban watersheds in the United States: U.S. Geological Survey Water-Supply Paper 2363, $44 \mathrm{p}$.

Duan, Naihua, 1983, Smearing estimate--a nonparametric retransformation method: Journal of the American Statistical Association, v. 78, no. 383, p. $605-610$.

Evaldi, R.D., Burns, R.J., and Moore, B.L., 1993, Water quality of selected streams in Jefferson County, Kentucky, 1988-91: U.S. Geological Survey Water-Resources Investigations Report 92-4150, 177 p.

Evaldi, R.D., and Moore, B.L., 1992, Stormwater data for Jefferson County, Kentucky, 1991-92: U.S. Geological Survey Open-File Report 92-638, 82 p.

Louisville Chamber of Commerce, 1992, Louisville fact book: Louisville, Ky., Louisville Chamber of Commerce Research Department, 199 p.

Melcher, N.B., and Ruh1, K.J., 1984, Streamflow and basin characteristics at selected sites in Kentucky: U.S. Geological Survey Open-File Report $84-704,80 \mathrm{p}$.

National Oceanic and Atmospheric Administration, 1990, Local climatological data, annual summary with comparative data, Louisville, Kentucky: Asheville, N.C., National Climatic Data Center, 8 p. 1991-92, Climatological data, Kentucky: Asheville, N.C., National Climatic Data Center (published monthly).

Rutledge, A.T., 1992, Methods of using streamflow records for estimating total and effective recharge in the Appalachian Valley and Ridge, Piedmont, and Blue Ridge physiographic provinces, in Hotchkiss, W.R., and Johnson, A.I., eds., Regional Aquifer Systems of the United States, Aquifers of the Southeastern Area--27th Annual Conference of American Water Resources Association, New Orleans, La., 1991: American Water Resources Association Monograph Series, no. 17, p. 59-73.

SAS Institute, Inc., 1985, SAS user's guide--statistics: Raleigh, N.C., $956 \mathrm{p}$.

Steurer, P.M., and Nold, Annett, 1986, Climatic data summaries from hourly precipitation data and state climatic divisions: National Oceanic and Atmospheric Administration, National Climatic Data Center, 41 p.

Strahler, A.N., and Strahler, A.H., 1979, Elements of physical geography (2d ed.): New York, John Wiley and Sons, 560 p. 
Tasker, G.D., Gilroy, E.J., and Jennings, M.E., 1990, Estimation of mean urban stormwater loads at unmonitored sites by regression, in Jennings, M.E., ed., Urban hydrology: Bethesda, Md., American Water Resources Association, p. 127-137.

U.S. Environmental Protection Agency, 1992, Guidance manual for the preparation of part 2 of the NPDES permit applications for discharges from municipal separate storm sewer systems: Washington, D.C., 158 p.

U.S. Geological Survey, 1986, Land use and land cover digital data from $1: 250,000$ and 1:100,000-scale maps: U.S. Geological Survey Data Users Guide 4, $36 \mathrm{p}$. 


\section{APPENDIX:}

SUPPLEMENTAL STREAMFLOW AND WATER-QUALITY INFORMATION 
Table 12. Average annual total streamflow and average annual base flow in streams in Jefferson County, Kentucky, 1988-92

$\left[f t^{3} / s\right.$, cubic feet per second]

\begin{tabular}{|c|c|c|c|c|}
\hline & \multirow[b]{2}{*}{ Site number and name } & \multirow{2}{*}{$\begin{array}{l}\text { Total } \\
\text { treamflow, } \\
\text { in } \mathrm{ft}^{3} / \mathrm{s}\end{array}$} & \multicolumn{2}{|c|}{ Base flow } \\
\hline & & & $\mathrm{ft}^{3} / \mathrm{s}$ & Percent \\
\hline 1. & Pond Creek at Pendleton Road & 96.2 & 39.9 & 41.5 \\
\hline & Mill Creek at Ore1l Road & 10.5 & 3.00 & 28.6 \\
\hline & Pond Creek at Manslick Road & 99.7 & 31.9 & 32.0 \\
\hline & South Fork Beargrass Creek at Winter Avenue & 33.5 & 11.0 & 32.8 \\
\hline 6 & South Fork Beargrass Creek at Trevilian Way & 24.0 & 9.30 & 38.8 \\
\hline & Middle Fork Beargrass Creek at Old Cannons Lane & 24.1 & 9.53 & 39.5 \\
\hline 81 & Middle Fork Beargrass Creek at Beals Branch Road & 29.1 & 9.71 & 33.4 \\
\hline & Spring Ditch at Private Drive & 4.26 & 2.04 & 47.9 \\
\hline 101 & Muddy Fork at Mockingbird Valley Road & 8.80 & 3.85 & 43.8 \\
\hline & Goose Creek at U.S. Highway 42 & 12.7 & 8.08 & 63.6 \\
\hline 12 & Little Goose Creek at U.S. Highway 42 & 9.97 & 5.70 & 57.2 \\
\hline 13 & Goose Creek at Old Westport Road & 9.87 & 6.38 & 64.6 \\
\hline 14 & Pope Lick at Pope Lick Road & 4.34 & 1.96 & 45.2 \\
\hline 15 & Floyds Fork at former State Highway 155 & 213 & 56.8 & 26.7 \\
\hline & Chenoweth Run at Gelhaus Road & 23.6 & 7.81 & 33.1 \\
\hline & Fern Creek at Old Bardstown Road & 6.78 & 3.18 & 46.9 \\
\hline & Northern Ditch at Preston Highway & 20.6 & 10.9 & 52.9 \\
\hline & Fishpool Creek at Bost Road & 8.24 & 2.82 & 34.2 \\
\hline & Southern Ditch at Minors Lane & 15.3 & 4.79 & 31.3 \\
\hline & Floyds Fork at Bardstown Road & 372 & 89.3 & 24.0 \\
\hline & Cedar Creek at Thixton Road & 16.8 & 6.98 & 41.5 \\
\hline & Pennsylvania Run at Mt. Washington Road & 9.15 & 3.88 & 42.4 \\
\hline 24 & Mill Creek Cutoff at Dover Road & 17.4 & 1.67 & 9.6 \\
\hline & Harrods Creek at Hunting Creek Drive & 131 & 59.5 & 45.4 \\
\hline 26 & Long Run at State Highway 1531 & 40.0 & 8.25 & 20.6 \\
\hline
\end{tabular}


Table 13. Statistical summary of constituent concentrations in streams in Jefferson County, Kentucky, $1988-92$

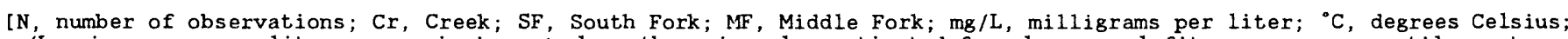
$\mu \mathrm{g} / \mathrm{L}$, micrograms per liter; ---, missing; <, less than; *, value estimated from log-normal fit program; percentiles not computed if fewer than 10 observations; the 10 th and 90 th percentiles are not shown if fewer than 30 observations]

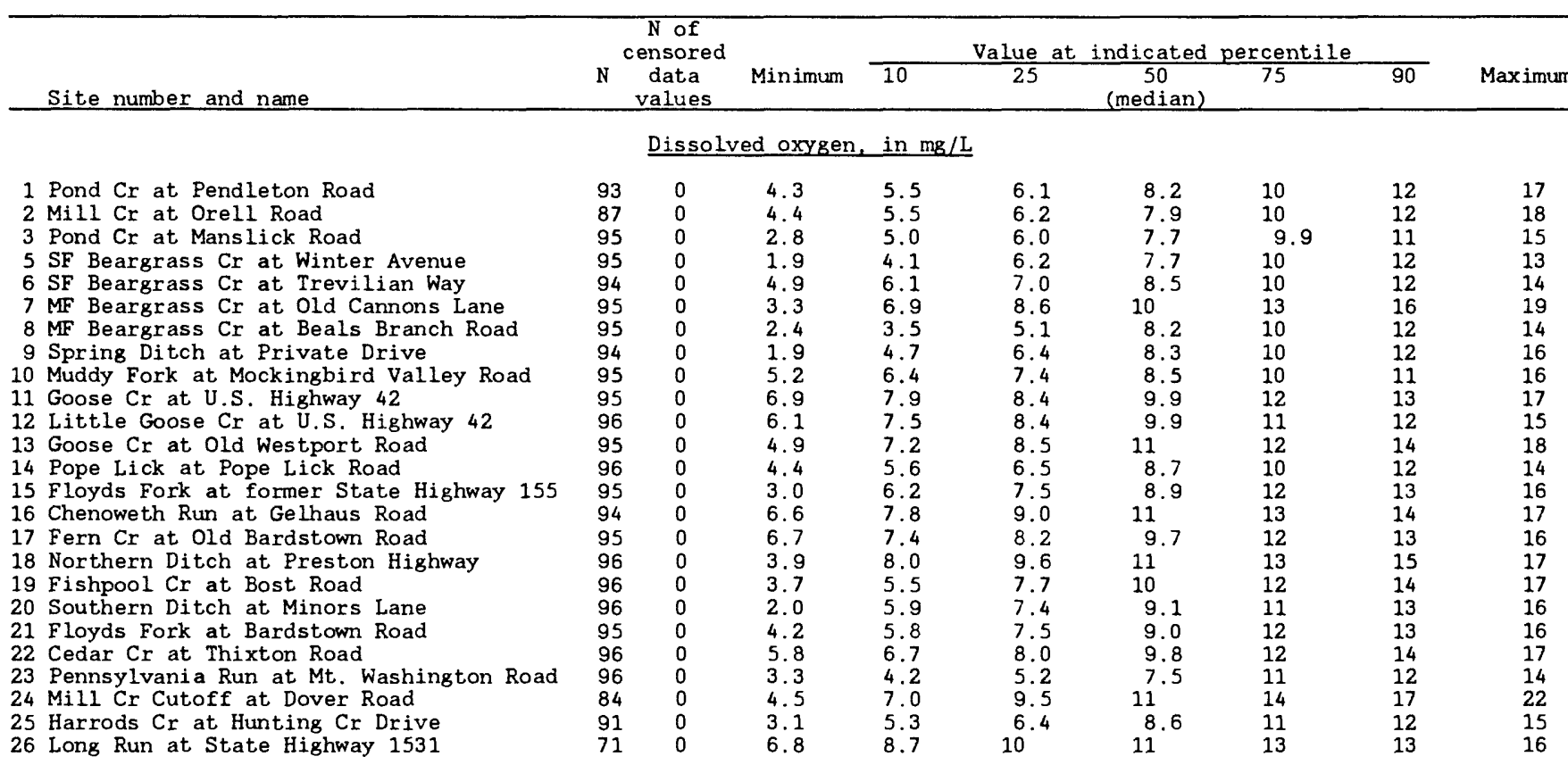

Chemical oxygen demand, $0.25 \mathrm{~N}$ dicromate, in $\mathrm{mg} / \mathrm{L}$

1 Pond $\mathrm{Cr}$ at Pendleton Road $2 \mathrm{Mill} \mathrm{Cr}$ at Orell Road

3 Pond $\mathrm{Cr}$ at Manslick Road

$5 \mathrm{SF}$ Beargrass $\mathrm{Cr}$ at Winter Avenue

6 SF Beargrass $\mathrm{Cr}$ at Trevilian Way

7 MF Beargrass $\mathrm{Cr}$ at Old Cannons Lane

$8 \mathrm{MF}$ Beargrass $\mathrm{Cr}$ at Beals Branch Road

9 Spring Ditch at Private Drive

10 Muddy Fork at Mockingbird Valley Road

11 Goose $\mathrm{Cr}$ at U.S. Highway 42

12 Little Goose $\mathrm{Cr}$ at U.S. Highway 42

13 Goose $\mathrm{Cr}$ at Old Westport Road

14 Pope Lick at Pope Lick Road

15 Floyds Fork at former State Highway 155

16 Chenoweth Run at Gelhaus Road

17 Fern $\mathrm{Cr}$ at Old Bardstown Road

18 Northern Ditch at Preston Highway

$19 \mathrm{Fishpoo} 1 \mathrm{Cr}$ at Bost Road

20 Southern Ditch at Minors Lane

21 Floyds Fork at Bardstown Road

22 Cedar $\mathrm{Cr}$ at Thixton Road

23 Pennsylvania Run at Mt. Washington Road

$24 \mathrm{Mill} \mathrm{Cr}$ Cutoff at Dover Road

25 Harrods $\mathrm{Cr}$ at Hunting $\mathrm{Cr}$ Drive

26 Long Run at State Highway 1531

$\begin{array}{lrlrrr}89 & 10 & <10 & 10^{*} & 14 & 20 \\ 84 & 11 & <10 & 9.5^{*} & 12 & 18 \\ 89 & 10 & <10 & 10^{*} & 14 & 20 \\ 88 & 26 & <10 & 5.7^{*} & 8.6^{*} & 14 \\ 89 & 37 & <10 & 4.4^{*} & 6.9^{*} & 11 \\ 89 & 36 & <10 & 5.1^{*} & 7.4^{*} & 12 \\ 90 & 29 & <10 & 6.0^{*} & 8.7^{*} & 14 \\ 90 & 10 & <10 & 10^{*} & 15 & 24 \\ 89 & 28 & <10 & 6.0^{*} & 8.8^{*} & 13 \\ 88 & 28 & <10 & 5.6^{*} & 8.5^{*} & 13 \\ 89 & 26 & <10 & 4.8^{*} & 7.7^{*} & 12 \\ 87 & 11 & <10 & 8.3^{*} & 12 & 17 \\ 92 & 13 & <10 & 8.4^{*} & 11 & 15 \\ 89 & 15 & <10 & 8.5^{*} & 11 & 18 \\ 89 & 15 & <10 & 8.8^{*} & 11 & 18 \\ 89 & 30 & <10 & 5.5^{*} & 8.3^{*} & 13 \\ 91 & 21 & <10 & 7.4^{*} & 11 & 18 \\ 89 & 15 & <10 & 8.9^{*} & 12 & 17 \\ 90 & 18 & <10 & 8.7^{*} & 13 & 20 \\ 90 & 20 & <10 & 7.2^{*} & 10 & 15 \\ 91 & 20 & <10 & 7.7^{*} & 10 & 17 \\ 92 & 10 & <10 & 11^{*} & 15 & 21 \\ 76 & 1 & <10 & 12.5 & 16 & 23 \\ 89 & 18 & <10 & 7.5^{*} & 11 & 17 \\ 63 & 15 & <10 & 7.0^{*} & 9.7^{*} & 15\end{array}$

$\begin{array}{rrr}26 & 33 & 45 \\ 25 & 31 & 49 \\ 25 & 38 & 55 \\ 21 & 31 & 60 \\ 20 & 32 & 81 \\ 18 & 23 & 41 \\ 21 & 26 & 84 \\ 37 & 50 & 141 \\ 24 & 30 & 64 \\ 20 & 31 & 70 \\ 19 & 33 & 710 \\ 26 & 36 & 138 \\ 22 & 28 & 59 \\ 23 & 30 & 94 \\ 24 & 30 & 61 \\ 21 & 29 & 74 \\ 24 & 33 & 103 \\ 25 & 32 & 47 \\ 26 & 33 & 105 \\ 24 & 31 & 70 \\ 23 & 31 & 101 \\ 28 & 33 & 49 \\ 31 & 40 & 105 \\ 24 & 40 & 55 \\ 20 & 26 & 49\end{array}$

Biochemical oxygen demand, 5-day at $20^{\circ} \mathrm{C}$, in $\mathrm{mg} / \mathrm{L}$

1 Pond $\mathrm{Cr}$ at Pendleton Road

$2 \mathrm{Mill} \mathrm{Cr}$ at Orell Road

3 Pond $\mathrm{Cr}$ at Manslick Road

5 SF Beargrass $\mathrm{Cr}$ at Winter Avenue

6 SF Beargrass $\mathrm{Cr}$ at Trevilian Way

7 MF Beargrass $\mathrm{Cr}$ at Old Cannons Lane

8 MF Beargrass $\mathrm{Cr}$ at Beals Branch Road

9 Spring Ditch at Private Drive

10 Muddy Fork at Mockingbird Valley Road

11 Goose $\mathrm{Cr}$ at U.S. Highway 42

12 Little Goose $\mathrm{Cr}$ at U.S. Highway 42

13 Goose $\mathrm{Cr}$ at Old Westport Road

14 Pope Lick at Pope Lick Road

15 Floyds Fork at former State Highway 155

16 Chenoweth Run at Gelhaus Road

17 Fern $\mathrm{Cr}$ at $01 d$ Bardstown Road

18 Northern Ditch at Preston Highway

$19 \mathrm{Fishpool} \mathrm{Cr}$ at Bost Road

20 Southern Ditch at Minors Lane

21 Floyds Fork at Bardstown Road

22 Cedar $\mathrm{Cr}$ at Thixton Road

$\begin{array}{rrr}89 & 20 & <2.0 \\ 85 & 20 & <2.0 \\ 90 & 8 & <2.0 \\ 91 & 14 & <2.0 \\ 91 & 39 & <2.0 \\ 91 & 34 & <2.0 \\ 92 & 35 & <2.0 \\ 91 & 12 & <2.0 \\ 90 & 35 & <2.0 \\ 90 & 27 & <2.0 \\ 91 & 32 & <2.0 \\ 90 & 18 & <2.0 \\ 92 & 18 & <2.0 \\ 91 & 18 & <2.0 \\ 90 & 14 & <2.0 \\ 91 & 21 & <2.0 \\ 92 & 17 & <2.0 \\ 91 & 29 & <2.0 \\ 91 & 13 & <2.0 \\ 90 & 24 & <2.0 \\ 92 & 34 & <1.7\end{array}$

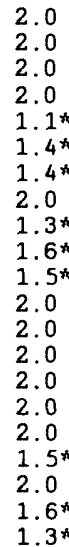

3. 0

3. 0

2. 0

2.0

2.0

2.0

2.0

4.0

3. 0

2.0

3.0

3.4

2. 0

3. 0

2.2
6.1

5.3
6.9

6.9

6.1

5.0

6.0

4.2
7.6

7.6
7.1

8.0

5.0

6.2
6.6

7.6

7.4

5.6

5.2

4.3

6.6
17
18
15
13
14
19
14
16
16
17
15
18
14
16
17
16
17
17
16
16
17
14
22
15
16 


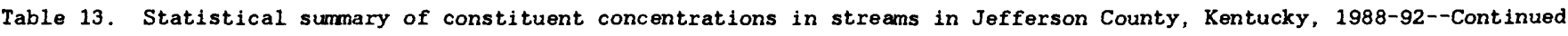

[N, number of observations; Cr, Creek; SF, South Fork; MF, Middle Fork; mg/L, milligrams per 1iter; ${ }^{\circ} \mathrm{C}$, degrees Celsius; 48/L, micrograms per liter; -- missing; <, less than; *, value estimated from log-normal fit program; percentiles not computed if fewer than 10 observations; the 10 th and 90 th percentiles are not shown if fewer than 30 observations]

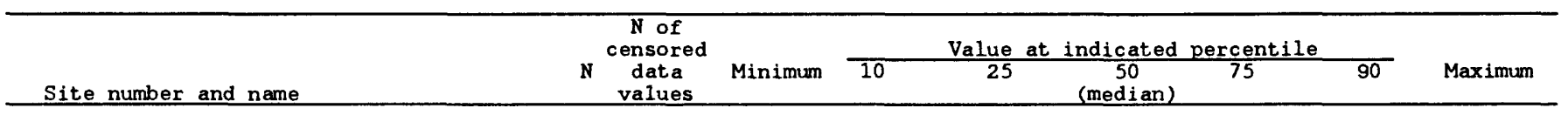

Biochemical oxygen demand, 5 -day at $20^{\circ} \mathrm{C}$, in $\mathrm{mg} / \mathrm{L}--$ Continued

23 Pennsylvania Run at Mt. Washington Road $24 \mathrm{Mill} \mathrm{Cr}$ Cutoff at Dover Road 25 Harrods $\mathrm{Cr}$ at Hunting $\mathrm{Cr}$ Drive 26 Long Run at State Highway 1531

\begin{tabular}{lrrrr}
92 & 14 & $<2.0$ & $1.4^{*}$ & 2.0 \\
79 & 7 & $<2.0$ & 2.0 & 3.0 \\
89 & 16 & $<2.0$ & $1.3^{*}$ & 2.0 \\
65 & 16 & $<2.0$ & $.99^{*}$ & $1.7^{*}$ \\
\multicolumn{7}{r}{ Calcium, total, in mg/L as Ca }
\end{tabular}

1 Pond $\mathrm{Cr}$ at Pendleton Road

$2 \mathrm{Mill} \mathrm{Cr}$ at Orell Road

3 Pond $\mathrm{Cr}$ at Manslick Road

5 SF Beargrass $\mathrm{Cr}$ at Winter Avenue

6 SF Beargrass $\mathrm{Cr}$ at Trevilian Way

7 MF Beargrass $\mathrm{Cr}$ at Old Cannons Lane

$8 \mathrm{MF}$ Beargrass $\mathrm{Cr}$ at Beals Branch Road

9 Spring Ditch at Private Drive

10 Muddy Fork at Mockingbird Valley Road

11 Goose $\mathrm{Cr}_{\mathrm{r}}$ at U.S. Hi ghway 42

12 Little Goose $\mathrm{Cr}$ at U.S. Highway 42

13 Goose $\mathrm{Cr}$ at Old Westport Road

14 Pope Lick at Pope Lick Road

15 Floyds Fork at former State Highway 155

16 Chenoweth Run at Gelhaus Road

17 Fern Cr at Old Bardstown Road

18 Northern Ditch at Preston Highway

$19 \mathrm{Fishpool} \mathrm{Cr}$ at Bost Road

20 Southern Ditch at Minors Lane

21 Floyds Fork at Bardstown Road

22 Cedar $\mathrm{Cr}$ at Thixton Road

23 Pennsylvania Run at Mt. Washington Road

$24 \mathrm{Mill} \mathrm{Cr}$ Cutoff at Dover Road

25 Harrods $\mathrm{Cr}$ at Hunting $\mathrm{Cr}$ Drive

26 Long Run at State Highway 1531

1 Pond $\mathrm{Cr}$ at Pendleton Road

$2 \mathrm{Mill} \mathrm{Cr}$ at Orell Road

3 Pond $\mathrm{Cr}$ at Manslick Road

5 SF Beargrass $\mathrm{Cr}$ at Winter Avenue

6 SF Beargrass $\mathrm{Cr}$ at Trevilian Way

7 MF Beargrass $\mathrm{Cr}$ at Old Cannons Lane

$8 \mathrm{MF}$ Beargrass $\mathrm{Cr}$ at Beals Branch Road

9 Spring Ditch at Private Drive

10 Muddy Fork at Mockingbird Valley Road

11 Goose $\mathrm{Cr}$ at U.S. Highway 42

12 Little Goose $\mathrm{Cr}$ at U.S. Highway 42

13 Goose $\mathrm{Cr}$ at Old Westport Road

14 Pope Lick at Pope Lick Road

15 Floyds Fork at former State Highway 155

16 Chenoweth Run at Gelhaus Road

17 Fern $\mathrm{Cr}$ at Old Bardstown Road

18 Northern Ditch at Preston Highway

$19 \mathrm{Fishpool} \mathrm{Cr}$ at Bost Road

20 Southern Ditch at Minors Lane

21 Floyds Fork at Bardstown Road

22 Cedar $\mathrm{Cr}$ at Thixton Road

23 Pennsylvania Run at Mt. Washington Road

$24 \mathrm{Mill} \mathrm{Cr}$ Cutoff at Dover Road

25 Harrods $\mathrm{Cr}$ at Hunting $\mathrm{Cr}$ Drive

26 Long Run at State Highway 1531

1 Pond $\mathrm{Cr}$ at Pendleton Road

$2 \mathrm{Mill} \mathrm{Cr}$ at Orell Road

3 Pond $\mathrm{Cr}$ at Manslick Road

5 SF Beargrass $C_{r}$ at Winter Avenue

6 SF Beargrass $\mathrm{Cr}$ at Trevilian Way

7 MF Beargrass $\mathrm{Cr}$ at Old Cannons Lane

$8 \mathrm{MF}$ Beargrass $\mathrm{Cr}$ at Beals Branch Road

9 Spring Ditch at Private Drive

10 Muddy Fork at Mockingbird Valley Road

11 Goose $\mathrm{Cr}$ at U.S. Highway 42

12 Little Goose $\mathrm{Cr}$ at U.S. Highway 42

13 Goose $\mathrm{Cr}$ at Old Westport Road

14 Pope Lick at Pope Lick Road

17
15
18
16
16
16
16
16
17
16
16
16
18
18
18
17
16
16
16
17
17
17
15
16
12

$\begin{array}{lll}43 & -- & 5 \\ 13 & -- & 3 \\ 47 & -- & 5 \\ 31 & -- & 44 \\ 35 & -- & 4 \\ 32 & -- & 58 \\ 45 & --- & 5 \\ 27 & -- & 59 \\ 58 & --- & 63 \\ 39 & -- & 52 \\ 43 & --- & 5 \\ 40 & -- & 49 \\ 52 & --- & 60 \\ 42 & --- & 54 \\ 40 & --- & 51 \\ 32 & --- & 55 \\ 41 & --- & 49 \\ 18 & --- & 51 \\ 36 & --- & 49 \\ 32 & --- & 53 \\ 20 & -- & 53 \\ 18 & --- & 39 \\ 19 & --- & 32 \\ 1.0 & --- & 47 \\ 48 & --- & 60\end{array}$

Magnesium, total, in $\mathrm{mg} / \mathrm{L}$ as $\mathrm{Mg}$

Alkalinity, in $\mathrm{mg} / \mathrm{L}$ as $\mathrm{CaCO}_{3}$
5.0

8.0

8.5

$\begin{array}{cc}9.7 & 20 \\ 12 & 18 \\ 10 & 13 \\ 12 & 14\end{array}$

\begin{tabular}{|c|c|c|c|c|c|c|c|c|}
\hline 17 & 0 & 14 & $\cdots$ & 18 & 21 & 24 & --- & 29 \\
\hline 15 & 0 & 3.2 & $-\cdots$ & 9.7 & 16 & 28 & -- & 33 \\
\hline 18 & 0 & 15 & $-\cdots$ & 20 & 22 & 23 & --- & 28 \\
\hline 16 & 0 & 4.9 & $\cdots$ & 9.9 & 13 & 16 & --- & 20 \\
\hline 16 & 0 & 7.1 & $-\cdots$ & 12 & 15 & 17 & --- & 20 \\
\hline 16 & 0 & 6.9 & $\cdots$ & 13 & 15 & 17 & --- & Is \\
\hline 16 & 0 & 9.7 & $-\cdots$ & 11 & 15 & 16 & --- & I \\
\hline 16 & 0 & 5.5 & $-\cdots$ & 13 & 16 & 19 & --- & 2 \\
\hline 17 & 0 & 7.5 & $-\cdots$ & 11 & 12 & 14 & --- & 20 \\
\hline 16 & 0 & 14 & -- & 21 & 22 & 24 & --- & 2 \\
\hline 16 & 0 & 13 & $-\cdots$ & 17 & 20 & 22 & --- & 23 \\
\hline 16 & 0 & 15 & -- & 19 & 22 & 24 & --- & 2 \\
\hline 18 & 0 & 19 & -- & 24 & 26 & 27 & --- & 3 \\
\hline 18 & 0 & 11 & -- & 13 & 17 & 20 & --- & 2 \\
\hline 17 & 0 & 14 & -- & 18 & 22 & 24 & --- & 27 \\
\hline 17 & 0 & 13 & $\cdots$ & 23 & 27 & 30 & -- & 32 \\
\hline 16 & 0 & 15 & -- & 19 & 21 & 25 & -- & 28 \\
\hline 16 & 0 & 4.7 & $-\cdots$ & 20 & 25 & 28 & --- & 3 \\
\hline 16 & 0 & 13 & $-\cdots$ & 22 & 25 & 27 & --- & 30 \\
\hline 17 & 0 & 9.5 & $\cdots$ & 15 & 18 & 21 & -- & 3 \\
\hline 17 & 0 & 7.3 & $\cdots$ & 22 & 28 & 35 & -- & 4] \\
\hline 17 & 0 & 8.7 & --- & 19 & 21 & 22 & --- & 2 \\
\hline 15 & 0 & 5.4 & $\cdots$ & 11 & 16 & 18 & --- & \\
\hline 16 & 0 & 18 & -- & 21 & 24 & 26 & --- & \\
\hline 12 & 0 & 7.4 & 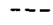 & 8.0 & 11 & 12 & --- & \\
\hline
\end{tabular}

$\begin{array}{lllrrrrrr}91 & 0 & 51 & 97 & 121 & 145 & 175 & 190 & 293 \\ 86 & 0 & 35 & 54 & 94 & 151 & 240 & 297 & 360 \\ 91 & 0 & 68 & 114 & 134 & 158 & 180 & 197 & 230 \\ 90 & 0 & 56 & 90 & 123 & 148 & 181 & 206 & 240 \\ 90 & 0 & 50 & 75 & 105 & 145 & 179 & 196 & 230 \\ 91 & 0 & 88 & 131 & 175 & 203 & 230 & 241 & 414 \\ 92 & 0 & 76 & 130 & 156 & 194 & 213 & 240 & 260 \\ 91 & 0 & 29 & 90 & 131 & 169 & 203 & 227 & 490 \\ 90 & 0 & 50 & 121 & 164 & 199 & 228 & 249 & 300 \\ 91 & 0 & 57 & 124 & 157 & 176 & 192 & 210 & 240 \\ 91 & 0 & 33 & 144 & 160 & 180 & 199 & 210 & 247 \\ 90 & 0 & 26 & 111 & 143 & 164 & 192 & 205 & 240 \\ 92 & 0 & 1.5 & 119 & 153 & 186 & 213 & 239 & 280\end{array}$




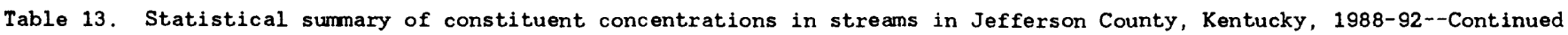

[N, number of observations; $\mathrm{Cr}$, Creek; SF, South Fork; MF, Middle Fork; mg/L, milligrams per liter; ${ }^{\circ} \mathrm{C}$, degrees Celsius; $\mu \mathrm{g} / \mathrm{L}$, micrograms per liter; ---, missing; <, less than; *, value estimated from log-normal fit program; percentiles not computed if fewer than 10 observations; the 10 th and 90 th percentiles are not shown if fewer than 30 observations]

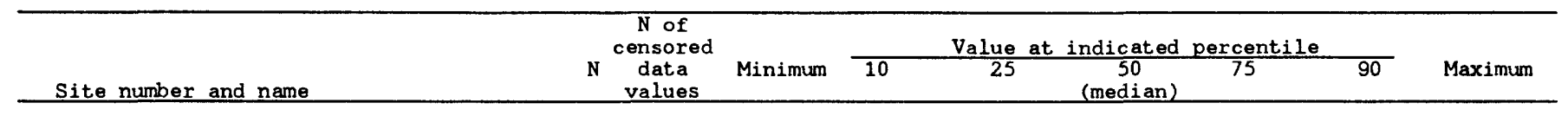

Alkalinity, in $\mathrm{mg} / \mathrm{L}$ as $\mathrm{CaCO}_{3}=-$ Continued

\begin{tabular}{|c|c|c|c|c|c|c|c|c|}
\hline $\begin{array}{l}15 \text { Floyds Fork at former State Highway } 155 \\
16 \text { Chenoweth Run at Gelhaus Road } \\
17 \text { Fern Cr at Old Bardstown Road } \\
18 \text { Northern Ditch at Preston Highway } \\
19 \text { Fishpool Cr at Bost Road } \\
20 \text { Southern Ditch at Minors Lane } \\
21 \text { Floyds Fork at Bardstown Road } \\
22 \text { Cedar Cr at Thixton Road } \\
23 \text { Pennsylvania Run at Mt. Washington Road } \\
24 \text { Mill Cr Cutoff at Dover Road } \\
25 \text { Harrods Cr at Hunting Cr Drive } \\
26 \text { Long Run at State Highway } 1531\end{array}$ & $\begin{array}{l}91 \\
90 \\
90 \\
92 \\
91 \\
91 \\
90 \\
92 \\
92 \\
78 \\
89 \\
65\end{array}$ & $\begin{array}{l}50 \\
2.0 \\
30 \\
61 \\
9.1 \\
46 \\
11 \\
8.8 \\
56 \\
42 \\
76 \\
90\end{array}$ & $\begin{array}{r}132 \\
100 \\
98 \\
120 \\
101 \\
99 \\
111 \\
99 \\
100 \\
66 \\
133 \\
136\end{array}$ & $\begin{array}{r}151 \\
123 \\
149 \\
149 \\
145 \\
147 \\
139 \\
136 \\
121 \\
96 \\
157 \\
164\end{array}$ & $\begin{array}{l}180 \\
171 \\
210 \\
184 \\
172 \\
164 \\
180 \\
173 \\
144 \\
123 \\
187 \\
180\end{array}$ & $\begin{array}{l}207 \\
200 \\
244 \\
200 \\
189 \\
183 \\
201 \\
200 \\
161 \\
162 \\
207 \\
205\end{array}$ & $\begin{array}{l}240 \\
220 \\
258 \\
219 \\
206 \\
198 \\
237 \\
220 \\
175 \\
210 \\
226 \\
240\end{array}$ & $\begin{array}{l}270 \\
290 \\
290 \\
260 \\
260 \\
237 \\
270 \\
265 \\
260 \\
279 \\
240 \\
250\end{array}$ \\
\hline
\end{tabular}

Dissolved solids, residue at $105^{\circ} \mathrm{C}$, in $\mathrm{mg} / \mathrm{L}$

1 Pond $\mathrm{Cr}$ at Pendleton Road

3 Pond $\mathrm{Cr}$ at Manslick Road

$5 \mathrm{SF}$ Beargrass $\mathrm{Cr}$ at Winter Avenue

$6 \mathrm{SF}$ Beargrass $\mathrm{Cr}$ at Trevilian Way

7 MF Beargrass $\mathrm{Cr}$ at Old Cannons Lane

$8 \mathrm{MF}$ Beargrass $\mathrm{Cr}$ at Beals Branch Road 9 Spring Ditch at Private Drive

10 Muddy Fork at Mockingbird Valley Road

11 Goose $\mathrm{Cr}$ at U.S. Highway 42

12 Little Goose $\mathrm{Cr}$ at U.S. Highway 42

13 Goose $\mathrm{Cr}$ at Old Westport Road

14 Pope Lick at Pope Lick Road

15 Floyds Fork at former State Highway 155

16 Chenoweth Run at Gelhaus Road

17 Fern $\mathrm{Cr}$ at Old Bardstown Road

18 Northern Ditch at Preston Highway

$19 \mathrm{Fishpool} \mathrm{Cr}$ at Bost Road

20 Southern Ditch at Minors Lane

21 Floyds Fork at Bardstown Road

22 Cedar $\mathrm{Cr}$ at Thixton Road

23 Pennsylvania Run at Mt. Washington Road

$24 \mathrm{Mill} \mathrm{Cr}$ Cutoff at Dover Road

25 Harrods $\mathrm{Cr}$ at Hunting $\mathrm{Cr}$ Drive

26 Long Run at State Highway 1531
2 Mill $\mathrm{Cr}$ at Orell Road

$\begin{array}{rrrrrr}91 & 0 & 134 & 238 & 315 & 37 \\ 86 & 0 & 34 & 159 & 196 & 263 \\ 91 & 0 & 116 & 286 & 333 & 394 \\ 91 & 0 & 21 & 232 & 290 & 345 \\ 91 & 0 & 116 & 226 & 291 & 323 \\ 91 & 0 & 161 & 250 & 304 & 35 \\ 91 & 0 & 124 & 234 & 292 & 351 \\ 91 & 0 & 36 & 232 & 304 & 394 \\ 90 & 0 & 221 & 355 & 408 & 45 \\ 91 & 0 & 156 & 231 & 292 & 33 \\ 91 & 0 & 110 & 250 & 306 & 340 \\ 90 & 0 & 166 & 226 & 272 & 319 \\ 91 & 0 & 133 & 279 & 343 & 39 \\ 91 & 0 & 199 & 238 & 266 & 305 \\ 89 & 0 & 176 & 306 & 338 & 394 \\ 90 & 0 & 123 & 305 & 377 & 41 \\ 91 & 0 & 121 & 282 & 337 & 40 \\ 90 & 0 & 114 & 286 & 360 & 41 \\ 88 & 0 & 160 & 291 & 345 & 40 \\ 90 & 0 & 164 & 235 & 275 & 31 \\ 91 & 0 & 158 & 273 & 368 & 46 \\ 92 & 0 & 149 & 217 & 266 & 32 \\ 79 & 0 & 92 & 174 & 243 & 328 \\ 89 & 0 & 154 & 232 & 276 & 310 \\ 64 & 0 & 92 & 205 & 239 & 279\end{array}$

Suspended solids, residue at $105^{\circ} \mathrm{C}$, in $\mathrm{mg} / \mathrm{L}$

1 Pond $\mathrm{Cr}$ at Pendleton Road

$2 \mathrm{Mill} \mathrm{Cr}$ at Orell Road

3 Pond $\mathrm{Cr}$ at Manslick Road

$5 \mathrm{SF}$ Beargrass $\mathrm{Cr}$ at Winter Avenue

$6 \mathrm{SF}$ Beargrass $\mathrm{Cr}$ at Trevilian Way

$7 \mathrm{MF}$ Beargrass $\mathrm{Cr}$ at Old Cannons Lane

$8 \mathrm{MF}$ Beargrass $\mathrm{Cr}$ at Beals Branch Road

9 Spring Ditch at Private Drive

10 Muddy Fork at Mockingbird Valley Road

11 Goose $\mathrm{Cr}$ at U.S. Highway 42

12 Little Goose $\mathrm{Cr}$ at U.S. Highway 42

13 Goose $\mathrm{Cr}$ at Old Westport Road

14 Pope Lick at Pope Lick Road

15 Floyds Fork at former State Highway 155

16 Chenoweth Run at Gelhaus Road

17 Fern $\mathrm{Cr}$ at Old Bardstown Road

18 Northern Ditch at Preston Highway

$19 \mathrm{~F}$ ishpool $\mathrm{Cr}$ at Bost Road

20 Southern Ditch at Minors Lane

21 Floyds Fork at Bardstown Road

22 Cedar $\mathrm{Cr}$ at Thixton Road

23 Pennsylvania Run at Mt. Washington Road

$24 \mathrm{Mill} \mathrm{Cr}$ Cutoff at Dover Road

25 Harrods $\mathrm{Cr}$ at Hunting $\mathrm{Cr}$ Drive

26 Long Run at State Highway 1531

$\begin{array}{rrrrr}91 & 0 & 2.0 & 5.0 & 8.0 \\ 86 & 0 & 2.0 & 4.0 & 7.0 \\ 91 & 0 & 4.0 & 14 & 27 \\ 90 & 0 & 2.0 & 6.0 & 9.7 \\ 91 & 0 & 2.0 & 5.0 & 8.0 \\ 91 & 0 & 1.0 & 3.0 & 5.0 \\ 91 & 0 & 1.0 & 4.0 & 5.0 \\ 91 & 0 & 1.0 & 5.2 & 11 \\ 90 & 0 & 2.0 & 5.0 & 10 \\ 91 & 0 & 2.0 & 4.2 & 7.0 \\ 91 & 0 & 3.0 & 4.4 & 8.0 \\ 90 & 0 & 1.0 & 6.0 & 8.9 \\ 92 & 0 & 1.0 & 4.0 & 7.0 \\ 91 & 0 & 2.0 & 4.2 & 8.0 \\ 89 & 0 & .70 & 2.0 & 5.0 \\ 90 & 0 & 1.5 & 5.0 & 7.0 \\ 92 & 0 & 1.0 & 4.0 & 7.0 \\ 91 & 0 & 1.6 & 4.8 & 7.0 \\ 90 & 1 & <1.0 & 5.0 & 11 \\ 90 & 1 & <1.0 & 3.1 & 7.0 \\ 91 & 1 & <1.0 & 3.0 & 5.0 \\ 92 & 0 & 1.0 & 4.0 & 7.0 \\ 79 & 0 & 2.0 & 4.0 & 6.0 \\ 89 & 0 & 4.0 & 10 & 14 \\ 65 & 0 & 2.0 & 3.0 & 5.0\end{array}$

17
48
20
15
8.0
10
18
18
12
17
18
11
16
8.0
13
11
12
19
15
7.0
14
13
28
10

52
31
95
40
28
16
24
36
39
29
28
42
20
30
16
27
26
27
32
27
16
22
25
45
20

113
92
143
71
175
42
65
86
115
66
85
118
64
102
48
66
56
72
51
106
42
57
42
103
61

500

4,350

1,370

3, 030

3,030
202

272

308

442

1,080

928
1,960

1,250

1,640

502

810

1,190

1,180

1,230

302

136

552

684
998

1,390

Residue, volatile nonfilterable, in $\mathrm{mg} / \mathrm{L}$

1 Pond $\mathrm{Cr}$ at Pendleton Road

$2 \mathrm{Mill} \mathrm{Cr}$ at Orell Road

3 Pond $\mathrm{Cr}$ at Manslick Road

5 SF Beargrass $\mathrm{Cr}$ at Winter Avenue

$6 \mathrm{SF}$ Beargrass $\mathrm{Cr}$ at Trevilian Way

$\begin{array}{lllll}91 & 2 & <1.0 & 2.0 & 3.0 \\ 86 & 4 & <.10 & 1.0 & 2.0 \\ 91 & 3 & <1.0 & 2.0 & 4.0 \\ 90 & 4 & <1.0 & 1.0 & 4.0 \\ 91 & 3 & <1.0 & 1.0 & 2.7\end{array}$

$\begin{array}{ll}5.0 & 10 \\ 4.1 & 11 \\ 9.0 & 20 \\ 6.0 & 16 \\ 5.0 & 12\end{array}$

326

510

556
382 


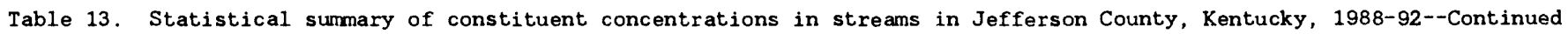

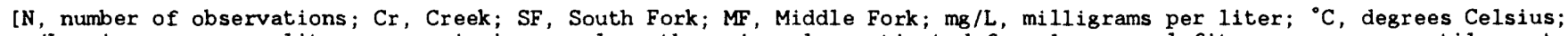
$\mu_{8} / \mathrm{L}$, micrograms per liter; ---, missing; <, less than; *, value estimated from log-normal fit program; percentiles not computed if fewer than 10 observations; the 10 th and 90 th percentiles are not shown if fewer than 30 observations]

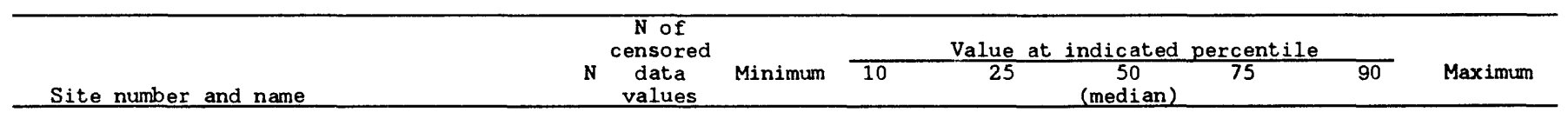

Residue, volatile nonfilterable, in mg/L--Continued

7 MF Beargrass $\mathrm{Cr}$ at Old Cannons Lane

$8 \mathrm{MF}$ Beargrass $\mathrm{Cr}$ at Beals Branch Road

9 Spring Ditch at Private Drive

10 Muddy Fork at Mockingbird Valley Road

11 Goose $\mathrm{Cr}$ at U.S. Highway 42

12 Little Goose $\mathrm{Cr}$ at U.S. Highway 42

13 Goose $\mathrm{Cr}$ at Old Westport Road

14 Pope Lick at Pope Lick Road

15 Floyds Fork at former State Highway 155

16 Chenoweth Run at Gelhaus Road

17 Fern $\mathrm{Cr}$ at OId Bardstown Road

18 Northern Ditch at Preston Highway

19 Fishpool $\mathrm{Cr}$ at Bost Road

20 Southern Ditch at Minors Lane

21 Floyds Fork at Bardstown Road

22 Cedar $\mathrm{Cr}$ at Thixton Road

23 Pennsylvania Run at Mt. Washington Road

24 Mill Cr Cutoff at Dover Road

25 Harrods $\mathrm{Cr}$ at Hunting $\mathrm{Cr}$ Drive

26 Long Run at State Highway 1531

91
91
91
90
91
91
90
92
91
89
90
92
91
90
90
91
92
79
89
65

Suspended solids, nonvolatile, in $\mathrm{mg} / \mathrm{L}$

1 Pond $\mathrm{Cr}$ at Pendleton Road

$2 \mathrm{Mill} \mathrm{Cr}$ at Orell Road

3 Pond $\mathrm{Cr}$ at Manslick Road

5 SF Beargrass $\mathrm{Cr}$ at Winter Avenue

6 SF Beargrass $\mathrm{Cr}$ at Trevilian Way

7 MF Beargrass $\mathrm{Cr}$ at Old Cannons Lane

8 MF Beargrass $\mathrm{Cr}$ at Beals Branch Road

9 Spring Ditch at Private Drive

10 Muddy Fork at Mockingbird Valley Road

11 Goose $\mathrm{Cr}$ at U.S. Highway 42

12 Little Goose $\mathrm{Cr}$ at U.S. Highway 42

13 Goose $\mathrm{Cr}$ at Old Westport Road

14 Pope Lick at Pope Lick Road

15 Floyds Fork at former State Highway 155

16 Chenoweth Run at Gelhaus Road

17 Fern $\mathrm{Cr}$ at O1d Bardstown Road

18 Northern Ditch at Preston Highway

$19 \mathrm{Fishpool} \mathrm{Cr}$ at Bost Road

20 Southern Ditch at Minors Lane

21 Floyds Fork at Bardstown Road

22 Cedar $\mathrm{Cr}$ at Thixton Road

23 Pennsylvania Run at Mt. Washington Road

24 Mill Cr Cutoff at Dover Road

25 Harrods $\mathrm{Cr}$ at Hunting $\mathrm{Cr}$ Drive

26 Long Run at State Highway 1531

$\begin{array}{rrrrr}20 & 0 & 1.0 & -- & 2.2 \\ 21 & 0 & 1.0 & -- & 2.0 \\ 21 & 0 & 1.0 & -- & 9.0 \\ 20 & 0 & <5.0 & -- & 5.0 \\ 20 & 0 & <3.0 & -- & 3.0 \\ 20 & 0 & 1.0 & -- & 1.0 \\ 21 & 0 & 1.0 & -- & 2.0 \\ 20 & 0 & 2.0 & --- & 4.0 \\ 19 & 0 & 1.0 & --- & 3.0 \\ 20 & 0 & 1.0 & -- & 3.2 \\ 20 & 0 & 2.0 & --- & 3.0 \\ 20 & 0 & 3.0 & --- & 7.5 \\ 20 & 0 & <3.0 & --- & 3.0 \\ 20 & 0 & 1.0 & --- & 2.2 \\ 19 & 0 & <1.0 & --- & 1.0 \\ 20 & 0 & 2.0 & --- & 3.0 \\ 21 & 0 & 1.0 & --- & 2.0 \\ 19 & 0 & 2.0 & --- & 3.0 \\ 20 & 0 & 2.0 & -- & 4.0 \\ 19 & 0 & <5.0 & --- & 5.0 \\ 20 & 0 & <2.0 & --- & 2.0 \\ 20 & 0 & 1.0 & --- & 2.1 \\ 19 & 0 & 1.0 & --- & 3.0 \\ 21 & 0 & 2.0 & --- & 5.5 \\ 14 & 0 & 1.0 & --- & 1.0 \\ & & & & \end{array}$

6.5

3.0

22

10

\section{5}

\section{0}

7.0

8.0
5.5

5.5
5.5

$$
16
$$

4.5

8. 0

3.0

3.0
4.5
4.0
5.0

4.0
5.0

8.5
9.0

9.0
5.0

5.0
4.0

4.0

12

12
2.5

Nitrogen, nitrate, total, in $\mathrm{mg} / \mathrm{L}$ as $\mathrm{N}$

1 Pond $\mathrm{Cr}$ at Pendleton Road

$2 \mathrm{Mill} \mathrm{Cr}$ at Orell Road

3 Pond $\mathrm{Cr}$ at Manslick Road

5 SF Beargrass $\mathrm{Cr}$ at Winter Avenue

6 SF Beargrass $\mathrm{Cr}$ at Trevilian Way

7 MF Beargrass $\mathrm{Cr}$ at Old Cannons Lane

8 MF Beargrass $\mathrm{Cr}$ at Beals Branch Road

9 Spring Ditch at Private Drive

10 Muddy Fork at Mockingbird Valley Road

11 Goose $\mathrm{Cr}$ at U.S. Highway 42

12 Little Goose $\mathrm{Cr}$ at U.S. Highway 42

13 Goose $\mathrm{Cr}$ at Old Westport Road

14 Pope Lick at Pope Lick Road

15 Floyds Fork at former State Highway 155

16 Chenoweth Run at Gelhaus Road

17 Fern $\mathrm{Cr}$ at Old Bardstown Road

18 Northern Ditch at Preston Highway

$19 \mathrm{Fishpool} \mathrm{Cr}$ at Bost Road

20 Southern Ditch at Minors Lane

21 Floyds Fork at Bardstown Road

22 Cedar $\mathrm{Cr}$ at Thixton Road

23 Pennsylvania Run at Mt. Washington Road

$24 \mathrm{Mill} \mathrm{Cr}$ Cutoff at Dover Road

25 Harrods $\mathrm{Cr}$ at Hunting $\mathrm{Cr}$ Drive

26 Long Run at State Highway 1531

$\begin{array}{lllll}91 & 2 & <.10 & .60 & 1.3 \\ 85 & 4 & <.10 & .11 & .23 \\ 91 & 3 & <.10 & .89 & 1.5 \\ 89 & 3 & <.10 & .57 & 1.0 \\ 89 & 3 & <.10 & .66 & .96 \\ 90 & 4 & <.10 & .62 & 1.5 \\ 91 & 4 & <.10 & .75 & 1.2 \\ 91 & 3 & <.10 & .48 & 1.1 \\ 89 & 3 & <.10 & 1.7 & 2.8 \\ 90 & 0 & .57 & 1.7 & 2.5 \\ 89 & 0 & .37 & 2.2 & 3.2 \\ 89 & 0 & .15 & 1.2 & 2.4 \\ 92 & 1 & <.10 & 1.1 & 2.1 \\ 90 & 3 & <.10 & .27 & .49 \\ 89 & 0 & .14 & 1.3 & 2.1 \\ 90 & 2 & <.10 & 1.7 & 2.8 \\ 92 & 4 & <.10 & 1.2 & 2.2 \\ 91 & 3 & <.10 & .87 & 1.7 \\ 91 & 3 & <.10 & .62 & 1.0 \\ 90 & 3 & <.10 & .33 & .82 \\ 92 & 0 & .11 & 1.2 & 1.9 \\ 92 & 1 & <.10 & .83 & 1.6 \\ 77 & 0 & .15 & .55 & 1.1 \\ 88 & 0 & .19 & .79 & 1.2 \\ 65 & 4 & <.10 & .11 & .24\end{array}$

2. 0

2.52

1.4

1.4
1.5

1.5
2.2

1.9

2.0

4.3

3.6

4.0

3.3

3. 1

3.7

4.5

3.5

2.8

1.9

1.3

2.9

2.8

1.9
1.7

1.7
.54

7.0
9.0
14
11
8.0
9.0
14
7.0
11
7.5
9.2
8.0
10
10
9.0
6.0
8.7
9.0
11
7.0

$\begin{array}{rr}20 & 331 \\ 19 & 114 \\ 27 & 96 \\ 17 & 144 \\ 25 & 88 \\ 20 & 435 \\ 24 & 310 \\ 16 & 190 \\ 32 & 134 \\ 18 & 348 \\ 24 & 232 \\ 19 & 136 \\ 21 & 138 \\ 18 & 968 \\ 20 & 116 \\ 12 & 90 \\ 15 & 79 \\ 18 & 156 \\ 20 & 534 \\ 15 & 152\end{array}$

331
114
96
144
88
435
310
190
134
348
232
136
138
968
116
90
79
156
534
152

$\begin{array}{ccr}22 & --- & 214 \\ 8.0 & --- & 106 \\ 44 & -- & 280 \\ 19 & -- & 110 \\ 15 & --- & 70 \\ 6.7 & --- & 24 \\ 6.0 & --- & 101 \\ 10 & --- & 19 \\ 12 & -- & 131 \\ 8.0 & --- & 115 \\ 15 & --- & 176 \\ 42 & -- & 250 \\ 16 & --- & 61 \\ 19 & --- & 160 \\ 4.0 & -- & 8.0 \\ 11 & --- & 352 \\ 6.5 & --- & 26 \\ 10 & --- & 54 \\ 16 & --- & 594 \\ 38 & --- & 210 \\ 12 & --- & 110 \\ 7.0 & --- & 54 \\ 9.0 & -- & 628 \\ 26 & --- & 916 \\ 5.0 & --- & 60 \\ & & \end{array}$

$\begin{array}{lcc}3.2 & 4.3 & 8.6 \\ 1.4 & 2.6 & 12 \\ 3.0 & 4.9 & 11 \\ 2.1 & 3.2 & 6.8 \\ 2.3 & 3.5 & 7.6 \\ 3.0 & 4.4 & 13 \\ 2.8 & 4.3 & 6.0 \\ 3.0 & 4.0 & 6.1 \\ 5.8 & 9.0 & 17 \\ 4.6 & 5.8 & 13 \\ 5.4 & 6.4 & 11 \\ 4.1 & 6.3 & 13 \\ 6.1 & 10 & 15 \\ 1.8 & 3.2 & 9.6 \\ 5.7 & 11 & 21 \\ 7.7 & 14 & 33 \\ 6.2 & 13 & 24 \\ 4.0 & 6.3 & 15 \\ 3.1 & 4.4 & 11 \\ 1.8 & 3.2 & 9.1 \\ 4.4 & 10 & 19 \\ 6.2 & 13 & 20 \\ 3.7 & 5.7 & 11 \\ 2.3 & 3.5 & 10 \\ 1.2 & 1.7 & 5.3\end{array}$


Table 13. Statistical summary of constituent concentrations in streams in Jefferson County, Kentucky, 1988-92--Continued

[N, number of observations; $\mathrm{Cr}$, Creek; SF, South Fork; MF, Middle Fork; mg/L, milligrams per 1iter; ${ }^{\circ} \mathrm{C}$, degrees Celsius; $\mu_{\mathrm{g}} / \mathrm{L}$, micrograms per liter; --- missing; <, less than; *, value estimated from log-normal fit program; percentiles not computed if fewer than 10 observations; the 10 th and 90th' percentiles are not shown if fewer than 30 observations]

\begin{tabular}{|c|c|c|c|c|c|c|c|c|c|c|}
\hline \multicolumn{2}{|r|}{ Site number and name } & $\mathbf{N}$ & $\begin{array}{l}\text { N of } \\
\text { ensored } \\
\text { data } \\
\text { values }\end{array}$ & Minimum & \multicolumn{4}{|c|}{ Value at indicated percentile } & $\overline{90}$ & Maximum \\
\hline \multicolumn{11}{|c|}{ Nitrogen, nitrite, total, in $\mathrm{mg} / \mathrm{L}$ as $\mathrm{N}$} \\
\hline & Pond $\mathrm{Cr}$ at Pendleton Road & 91 & 2 & $<0.01$ & 0.01 & 0.03 & 0.06 & 0.08 & 0.12 & 0.57 \\
\hline & Mill $\mathrm{Cr}$ at Orell Road & 86 & 22 & $<.01$ & $<.01 *$ & $.01^{\star}$ & .02 & .04 & .08 & 6.0 \\
\hline & Pond $\mathrm{Cr}$ at Manslick Road & 91 & 0 & .01 & .04 & .06 & .08 & .12 & 20 & 1.1 \\
\hline & $\mathrm{SF}$ Beargrass $\mathrm{Cr}$ at Winter Avenue & 90 & 3 & $<.01$ & .02 & .03 & .06 & .08 & 11 & .14 \\
\hline & SF Beargrass Cr at Trevilian Way & 90 & 3 & $<.01$ & .01 & .02 & .05 & .06 & .08 & .43 \\
\hline & MF Beargrass $\mathrm{Cr}$ at Old Cannons Lane & 90 & 14 & $<.01$ & $.01^{*}$ & .01 & .02 & .03 & .05 & .21 \\
\hline & MF Beargrass Cr at Beals Branch Road & 91 & 11 & $<.01$ & $.01^{*}$ & .01 & .02 & .03 & .05 & .30 \\
\hline & Spring Ditch at Private Drive & 91 & 3 & $<.01$ & .02 & .03 & .04 & .06 & .10 & .71 \\
\hline 10 & Muddy Fork at Mockingbird Valley Road & 90 & 1 & $<.01$ & .02 & .02 & .04 & .06 & .09 & .58 \\
\hline 11 & Goose $\mathrm{Cr}$ at U.S. Highway 42 & 91 & 13 & $<.01$ & $.01^{\star}$ & .01 & .03 & .05 & 10 & 1.5 \\
\hline 12 & Little Goose $\mathrm{Cr}$ at U.S. Highway 42 & 91 & 8 & $<.01$ & $.01^{*}$ & .01 & .03 & .04 & .09 & .37 \\
\hline 13 & Goose $\mathrm{Cr}$ at Old Westport Road & 90 & 3 & $<.01$ & .02 & .03 & .05 & .11 & .27 & .45 \\
\hline 14 & Pope Lick at Pope Lick Road & 92 & 0 & .01 & .03 & .05 & .07 & .12 & 23 & 1.3 \\
\hline 15 & Floyds Fork at former State Highway 155 & 91 & 17 & $<.01$ & $.01^{*}$ & .01 & .02 & .04 & .06 & .31 \\
\hline & Chenoweth Run at Gelhaus Road & 90 & 5 & $<.01$ & .01 & .03 & .07 & .19 & 32 & .45 \\
\hline 17 & Fern $\mathrm{Cr}$ at Old Bardstown Road & 91 & 2 & $<.01$ & .02 & .03 & .05 & .10 & .22 & 1.8 \\
\hline 18 & Northern Ditch at Preston Highway & 92 & 0 & .01 & .03 & .04 & .07 & .12 & .23 & .71 \\
\hline 19 & Fishpool $\mathrm{Cr}$ at Bost Road & 91 & 5 & $<.01$ & .02 & .04 & .06 & .13 & .21 & .91 \\
\hline 20 & Southern Ditch at Minors Lane & 91 & 1 & $<.01$ & .02 & .04 & .07 & .11 & 15 & .36 \\
\hline & Floyds Fork at Bardstown Road & 90 & 12 & $<.01$ & $.01 *$ & .01 & .02 & .03 & .09 & .17 \\
\hline 22 & Cedar $\mathrm{Cr}$ at Thixton Road & 92 & 7 & $<.01$ & .01 & .01 & .03 & .05 & .08 & .35 \\
\hline 23 & Pennsylvania Run at Mt. Washington Road & 92 & 2 & $<.01$ & .02 & .04 & .08 & .18 & .28 & .81 \\
\hline & Mill Cr Cutoff at Dover Road & 79 & 10 & $<.01$ & $.01 *$ & .03 & .06 & .11 & .21 & .90 \\
\hline 25 & Harrods $\mathrm{Cr}$ at Hunting $\mathrm{Cr}$ Drive & 89 & 13 & $<.01$ & $.01 *$ & .01 & .02 & .04 & .06 & 1.5 \\
\hline 26 & Long Run at State Highway 1531 & 67 & 23 & $<.01$ & $<.01^{*}$ & $.01 *$ & .01 & .02 & .04 & .24 \\
\hline
\end{tabular}

1 Pond $\mathrm{Cr}$ at Pendleton Road
2 Mill $\mathrm{Cr}$ at Orell Road
3 Pond Cr at Manslick Road
5 SF Beargrass $\mathrm{Cr}$ at Winter Avenue
$6 \mathrm{SF}$ Beargrass $\mathrm{Cr}$ at Trevilian Way
$7 \mathrm{MF}$ Beargrass $\mathrm{Cr}$ at Old Cannons Lane
$8 \mathrm{MF}$ Beargrass $\mathrm{Cr}$ at Beals Branch Road
$9 \mathrm{Spring}$ Ditch at Private Drive
10 Muddy Fork at Mockingbird Valley Road
11 Goose Cr at U.S. Highway 42
12 Little Goose Cr at U.S. Highway 42
13 Goose Cr at Old Westport Road
14 Pope Lick at Pope Lick Road
15 Floyds Fork at former State Highway 155
16 Chenoweth Run at Gelhaus Road
17 Fern Cr at O1d Bardstown Road
18 Northern Ditch at Preston Highway
19 Fishpool Cr at Bost Road
20 Southern Ditch at Minors Lane
21 Floyds Fork at Bardstown Road
22 Cedar Cr at Thixton Road
23 Pennsylvania Run at Mt. Washington Road
24 Mill Cr Cutoff at Dover Road
25 Harrods Cr at Hunting Cr Drive
26 Long Run at State Highway 1531

Nitrogen, ammonia, total, in $\mathrm{mg} / \mathrm{L}$ as $\mathrm{N}$

$\begin{array}{lllllllll}89 & 28 & <.01 & <.01^{*} & .01^{*} & .11 & .28 & .49 & 1.1 \\ 84 & 35 & <.01 & <.01^{*} & .01^{*} & .04 & .11 & .18 & .70 \\ 89 & 23 & <.01 & .01^{*} & .02^{*} & .25 & .45 & .62 & 5.3 \\ 89 & 25 & <.01 & <.01^{*} & .02^{*} & .17 & .32 & .56 & 2.4 \\ 89 & 22 & <.01 & .01^{*} & .02^{*} & .22 & .34 & .73 & 5.6 \\ 89 & 39 & <.01 & <.01^{*} & <.01^{*} & .04 & .11 & .22 & 4.5 \\ 90 & 40 & <.01 & <.01^{*} & <.01^{*} & .04 & .11 & .28 & 4.6 \\ 88 & 32 & <.01 & <.01^{*} & .01^{*} & .10 & .34 & .50 & .95 \\ 89 & 38 & <.01 & <.01^{*} & <.01^{*} & .06 & .14 & .22 & .64 \\ 91 & 43 & <.01 & <.01^{*} & <.01^{*} & .04 & .11 & .42 & 3.2 \\ 90 & 43 & <.01 & <.01^{*} & <.01^{*} & .02 & .09 & .20 & .71 \\ 89 & 35 & <.01 & <.01^{*} & .01^{*} & .10 & .45 & .78 & 1.3 \\ 92 & 27 & <.01 & <.01^{*} & .01^{*} & .13 & .33 & .77 & 3.5 \\ 91 & 39 & <.01 & <.01^{*} & <.01^{*} & .04 & .09 & .37 & 6.6 \\ 88 & 31 & <.01 & <.01^{*} & .01^{*} & .09 & .36 & .76 & 6.8 \\ 89 & 25 & <.01 & <.01^{*} & .01^{*} & .11 & .32 & .67 & 1.8 \\ 89 & 28 & <.01 & <.01^{*} & .01^{*} & .16 & .42 & 1.6 & 9.1 \\ 88 & 33 & <.01 & <.01^{*} & .01^{*} & .07 & .22 & .45 & .98 \\ 88 & 29 & <.01 & <.01^{*} & .01^{*} & .11 & .24 & .45 & 3.6 \\ 90 & 36 & <.01 & <.01^{*} & .01^{*} & .06 & .11 & .35 & 2.3 \\ 91 & 40 & <.01 & <.01^{*} & <.01^{*} & .06 & .12 & .38 & 4.0 \\ 90 & 20 & <.01 & .01^{*} & .04^{*} & .20 & .39 & .69 & 2.8 \\ 78 & 33 & <.01 & <.01^{*} & <.01^{*} & .06 & .22 & .66 & 3.4 \\ 89 & 46 & <.01 & <.01^{*} & <.01^{*} & .01 * & .10 & .19 & .78 \\ 65 & 35 & <.01 & <.01^{*} & <.01^{*} & .01^{*} & .08 & .24 & 1.4\end{array}$

1 Pond $\mathrm{Cr}$ at Pendleton Road

$2 \mathrm{Mill} \mathrm{Cr}$ at Orell Road

3 Pond $\mathrm{Cr}$ at Manslick Ro

$5 \mathrm{SF}$ Beargrass $\mathrm{Cr}$ at Winter Avenue

$6 \mathrm{SF}$ Beargrass $\mathrm{Cr}$ at Trevilian Way

7 MF Beargrass $\mathrm{Cr}$ at Old Cannons Lane

$8 \mathrm{MF}$ Beargrass $\mathrm{Cr}$ at Beals Branch Road

9 Spring Ditch at Private Drive

10 Muddy Fork at Mockingbird Valley Road

11 Goose $\mathrm{Cr}$ at U.S. Highway 42

12 Little Goose $\mathrm{Cr}$ at U.S. Highway 42

13 Goose $\mathrm{Cr}$ at old Westport Road

14 Pope Lick at Pope Lick Road

15 Floyds Fork at former State Highway 155

16 Chenoweth Run at Gelhaus Road

17 Fern $\mathrm{Cr}$ at Old Bardstown Road

18 Northern Ditch at Preston Highway

$19 \mathrm{Fishpool} \mathrm{Cr}$ at Bost Road

20 Southern Ditch at Minors Lane

21 Floyds Fork at Bardstown Road

22 Cedar $\mathrm{Cr}$ at Thixton Road
Nitrogen, organic, dissolved, in $\mathrm{mg} / \mathrm{L}$

$\begin{array}{lllllllll}91 & 14 & <.05 & .06^{*} & .24 & .51 & .70 & 1.1 & 1.8 \\ 87 & 12 & <.05 & .04^{*} & .11 & .31 & .67 & .97 & 6.9 \\ 91 & 13 & <.05 & .09^{*} & .40 & .67 & .90 & 1.3 & 2.5 \\ 92 & 16 & <.05 & .06^{*} & .22 & .47 & .78 & 1.2 & 2.0 \\ 92 & 16 & <.05 & .05^{*} & .22 & .39 & .67 & 1.0 & 2.5 \\ 92 & 23 & <.05 & .03^{*} & .06^{*} & .32 & .48 & .89 & 3.5 \\ 93 & 25 & <.05 & .02^{*} & .06^{*} & .28 & .51 & .86 & 3.1 \\ 91 & 13 & <.05 & .06^{*} & .19 & .64 & .91 & 1.3 & 2.9 \\ 91 & 21 & <.05 & .03^{*} & .07^{*} & .31 & .56 & .75 & 4.0 \\ 91 & 22 & <.05 & .03^{*} & .08^{*} & .43 & .56 & .86 & 6.9 \\ 90 & 21 & <.05 & .03^{*} & .07^{*} & .39 & .62 & .90 & 2.7 \\ 91 & 20 & <.05 & .04^{*} & .13 & .65 & .95 & 1.4 & 4.8 \\ 93 & 10 & <.05 & .10^{*} & .32 & .60 & .78 & 1.2 & 3.6 \\ 91 & 11 & <.05 & .07^{*} & .17 & .44 & .73 & 1.0 & 3.4 \\ 91 & 20 & <.05 & .03^{*} & .09^{*} & .45 & .80 & 1.2 & 3.9 \\ 92 & 12 & <.05 & .07^{*} & .20 & .50 & .84 & 1.2 & 4.2 \\ 92 & 15 & <.05 & .05^{*} & .11 & .62 & .95 & 1.5 & 4.8 \\ 90 & 13 & <.05 & .07^{*} & .22 & .62 & .84 & 1.3 & 1.8 \\ 91 & 14 & <.05 & .06^{*} & .17 & .64 & .84 & 1.1 & 1.9 \\ 90 & 13 & <.05 & .07^{*} & .22 & .50 & .84 & 1.1 & 1.3 \\ 93 & 10 & <.05 & .09^{*} & .26 & .50 & .73 & 1.1 & 2.0\end{array}$




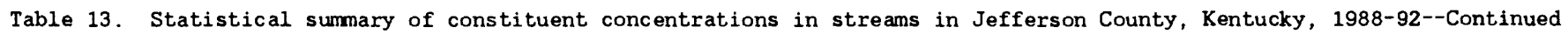

[N, number of observations; $\mathrm{Cr}$, Creek; SF, South Fork; MF, Middle Fork; mg/L, milligrams per liter; ${ }^{\circ} \mathrm{C}$, degrees Celsius; $\mu \mathrm{g} / \mathrm{L}$, micrograms per liter; --- missing; <, less than; *, value estimated from log-normal fit program; percentiles not computed if fewer than 10 observations; the 10 th and 90 th percentiles are not shown if fewer than 30 observations]

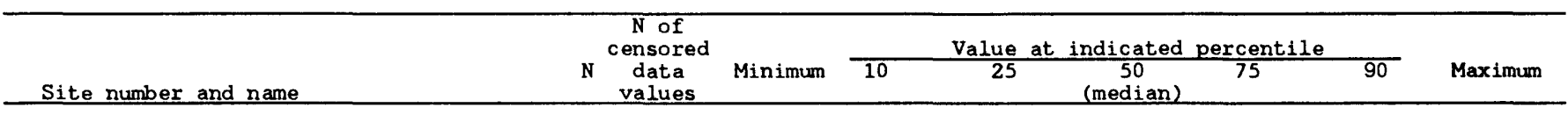

Nitrogen, organic, dissolved, in $\mathrm{mg} / \mathrm{L}--$ Continued

23 Pennsylvania Run at Mt. Washington Road $24 \mathrm{Mill} \mathrm{Cr}$ Cutoff at Dover Road

25 Harrods $\mathrm{Cr}$ at Hunting $\mathrm{Cr}$ Drive

26 Long Run at State Highway 1531

1 Pond $\mathrm{Cr}$ at Pendleton Road

2 Mill $\mathrm{Cr}$ at Orell Road

3 Pond $\mathrm{Cr}$ at Manslick Road

5 SF Beargrass $\mathrm{Cr}$ at Winter Avenue

6 SF Beargrass $\mathrm{Cr}$ at Trevilian Way

7 MF Beargrass $\mathrm{Cr}$ at Old Cannons Lane

8 MF Beargrass $\mathrm{Cr}$ at Beals Branch Road

9 Spring Ditch at Private Drive

10 Muddy Fork at Mockingbird Valley Road

11 Goose $\mathrm{Cr}$ at U.S. Highway 42

12 Little Goose $\mathrm{Cr}$ at U.S. Highway 42

13 Goose $\mathrm{Cr}$ at Old Westport Road

14 Pope Lick at Pope Lick Road

15 Floyds Fork at former State Highway 155

16 Chenoweth Run at Gelhaus Road

17 Fern $\mathrm{Cr}$ at Old Bardstown Road

18 Northern Ditch at Preston Highway

19 Fishpool $\mathrm{Cr}$ at Bost Road

20 Southern Ditch at Minors Lane

21 Floyds Fork at Bardstown Road

22 Cedar $\mathrm{Cr}$ at Thixton Road

23 Pennsylvania Run at Mt. Washington Road

$24 \mathrm{Mill} \mathrm{Cr}$ Cutoff at Dover Road

25 Harrods $\mathrm{Cr}$ at Hunting $\mathrm{Cr}$ Drive

26 Long Run at State Highway 1531

1 Pond $\mathrm{Cr}$ at Pendleton Road

$2 \mathrm{Mill} \mathrm{Cr}$ at Orell Road

3 Pond $\mathrm{Cr}$ at Manslick Road

5 SF Beargrass $\mathrm{Cr}$ at Winter Avenue

6 SF Beargrass $\mathrm{Cr}$ at Trevilian Way

7 MF Beargrass $\mathrm{Cr}$ at Old Cannons Lane

8 MF Beargrass $\mathrm{Cr}$ at Beals Branch Road

9 Spring Ditch at Private Drive

10 Muddy Fork at Mockingbird Valley Road

11 Goose $\mathrm{Cr}$ at U.S. Highway 42

12 Little Goose $\mathrm{Cr}$ at U.S. Highway 42

13 Goose $\mathrm{Cr}$ at Old Westport Road

14 Pope Lick at Pope Lick Road

15 Floyds Fork at former State Highway 155

16 Chenoweth Run at Gelhaus Road

17 Fern $\mathrm{Cr}$ at Old Bardstown Road

18 Northern Ditch at Preston Highway

$19 \mathrm{Fishpool} \mathrm{Cr}$ at Bost Road

20 Southern Ditch at Minors Lane

21 Floyds Fork at Bardstown Road

22 Cedar $\mathrm{Cr}$ at Thixton Road

23 Pennsylvania Run at Mt. Washington Road

$24 \mathrm{Mill} \mathrm{Cr}$ Cutoff at Dover Road

25 Harrods $\mathrm{Cr}$ at Hunting $\mathrm{Cr}$ Drive

26 Long Run at State Highway 1531 $\begin{array}{ll}92 & 16 \\ 80 & 14 \\ 90 & 13 \\ 66 & 8\end{array}$

$\begin{array}{rr}<0.05 & 0.06 * \\ <.05 & .06 * \\ <.05 & .06 * \\ <.05 & .07 *\end{array}$

0.22
.22
.12
.18

0.65
.62
.42

0.90
.95
.65
.67

1.4
1.3
1.0

2. 6

.39

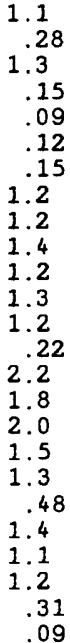

1.7

2.49

.25

.18

.22
.28

1.8

1.8
2.0
2.8

2. 4

2.7

3.1

.35

.13

1.1

1.1
1.2

.95

.80

31
83

.56

.49

.06

2.2

2.2
1.8

2.0

1. 3

.48

1. 1

.31

.03

3.7
3.5

3. 6

3. 1

2.4

.86

2.7

2.7
2.7
1.8

1. 8

.63
.18

3.0

3.86

.47
.25

.25
.31

2.76

2. 7

3.9

4.0

3. 8

4.4
4.3

.58

5. 5

5. 4

4.3

4.3
4.1
3.5

1. 6

4.2

4. 9

3.4

1.4
.43

.88

3. 4

Phosphorus, total, in $\mathrm{mg} / \mathrm{L}$ as $\mathrm{P}$

$\begin{array}{lllll}88 & 0 & .12 & .33 & .39 \\ 83 & 1 & <.01 & .08 & .11 \\ 86 & 0 & .11 & .37 & .50 \\ 89 & 0 & .02 & .05 & .08 \\ 87 & 0 & .02 & .03 & .07 \\ 88 & 0 & .01 & .04 & .06 \\ 89 & 0 & .02 & .04 & .08 \\ 86 & 0 & .11 & .34 & .51 \\ 88 & 0 & .13 & .30 & .48 \\ 88 & 0 & .15 & .28 & .56 \\ 88 & 0 & .09 & .24 & .45 \\ 89 & 0 & .12 & .33 & .56 \\ 88 & 0 & .09 & .34 & .52 \\ 86 & 0 & .03 & .07 & .11 \\ 86 & 0 & .15 & .34 & .80 \\ 89 & 0 & .19 & .58 & .75 \\ 89 & 0 & .14 & .47 & .79 \\ 87 & 0 & .09 & .35 & .58 \\ 89 & 0 & .10 & .32 & .52 \\ 86 & 0 & .05 & .13 & .23 \\ 89 & 0 & .12 & .33 & .58 \\ 90 & 0 & .15 & .22 & .46 \\ 78 & 0 & .05 & .21 & .47 \\ 87 & 0 & .03 & .09 & .13 \\ 65 & 0 & .03 & .04 & .06\end{array}$

.59

1.1

1.2

1.2
.24

.13

.23

1.1

1.7

1.1

.96

1.5

1. 2

1. 2

. 17

1.7

1.7
1.4

1. 1

.90

.35

1. 0

1.0
.74

.74
.26
.11

2.0

2. 1

.28

2.6

2.6
2.1

2.1
1.7

1.6

.79

1. 8

2.2
1.3

1. 3

.55
.22

4.2

$\begin{array}{lr}1.6 & 2 . \\ 4.5 & 16\end{array}$

1.23 .2

$.61 \quad 10$

$\begin{array}{ll}.64 & 7.3 \\ .71 & 1.8\end{array}$

4.2

4. 6

5.1

5. 4

6.0

6.3

1. 1

7.7

7.2

6.8

5.2

4.4

3.4

5. 3

4.

2.2

.86

9.2

16

11

12

3. 6

23

24
17

14

14

13

23

5.2

14

$\begin{array}{lr}2.0 & 7.4 \\ .84 & 2.5 \\ 2.1 & 9.1 \\ .65 & 1.9 \\ .28 & 3.7 \\ .34 & 1.7 \\ .40 & 1.6 \\ 1.9 & 4.1 \\ 2.3 & 18 \\ 2.1 & 6.1 \\ 2.5 & 7.2 \\ 2.7 & 5.8 \\ 2.8 & 9.8 \\ .48 & 1.5 \\ 3.5 & 12 \\ 3.5 & 32 \\ 3.1 & 9.9 \\ 2.4 & 6.8 \\ 2.0 & 6.8 \\ 1.3 & 5.8 \\ 2.6 & 6.0 \\ 2.7 & 8.0 \\ 2.2 & 10 \\ 1.0 & 2.6 \\ 58 & 5.9 \\ & \end{array}$

Phosphorus, orthophosphate, total, in mg/L as P

1 Pond $\mathrm{Cr}$ at Pendleton Road

$2 \mathrm{Mill} \mathrm{Cr}$ at Orell Road

3 Pond $\mathrm{Cr}$ at Manslick Road

$5 \mathrm{SF}$ Beargrass $\mathrm{Cr}$ at Winter Avenue

6 SF Beargrass $\mathrm{Cr}$ at Trevilian Way

7 MF Beargrass $\mathrm{Cr}$ at Old Cannons Lane

$8 \mathrm{MF}$ Beargrass $\mathrm{Cr}$ at Beals Branch Road

9 Sprins Ditch at Private Drive

10 Muddy Fork at Mockingbird Valley Road

11 Goose $\mathrm{Cr}$ at U.S. Highway 42

12 Little Goose $\mathrm{Cr}$ at U.S. Highway 42

13 Goose $\mathrm{Cr}$ at Old Westport Road

14 Pope Lick at Pope Lick Road

$\begin{array}{rrrrr}91 & 0 & .11 & .28 & .35 \\ 85 & 1 & <.01 & .06 & .09 \\ 91 & 0 & .11 & .30 & .44 \\ 90 & 0 & .01 & .03 & .05 \\ 90 & 1 & <.01 & .01 & .03 \\ 89 & 4 & <.01 & .01 & .03 \\ 90 & 3 & <.01 & .02 & .05 \\ 90 & 2 & <.01 & .21 & .37 \\ 89 & 0 & .04 & .21 & .40 \\ 90 & 0 & .05 & .24 & .46 \\ 91 & 0 & .08 & .18 & .39 \\ 89 & 0 & .05 & .23 & .42 \\ 91 & 0 & .03 & .24 & .39\end{array}$

2.9
3.2
7.3
1.8
9.2
6
3
1
12
3
3.6
3
4
7
4
13
3
5
11
5.2
4


Table 13. Statistical summary of constituent concentrations in streams in Jefferson County, Kentucky, 1988-92--Continued

[N, number of observations; $\mathrm{Cr}$, Creek; SF, South Fork; MF, Middle Fork; mg/L, milligrams per liter; ${ }^{\circ} \mathrm{C}$, degrees Celsius; $\mu_{8} / L$, micrograms per liter; ---, missing; <, less than; *, value estimated from log-normal fit program; percentiles not computed if fewer than 10 observations; the 10 th and 9oth percentiles are not shown if fewer than 30 observations]

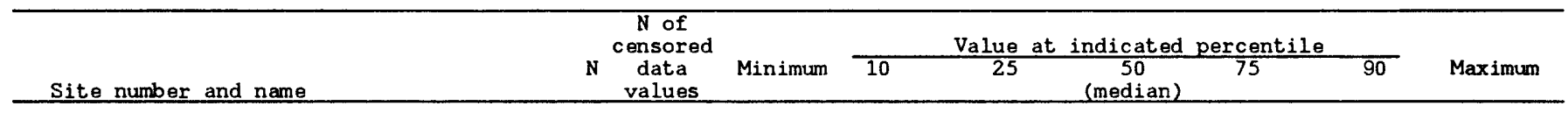

Phosphorus, orthophosphate, total, in $\mathrm{mg} / \mathrm{L}$ as $\mathrm{P}$--Continued

15 Floyds Fork at former State Highway 155
16 Chenoweth Run at Gelhaus Road
17 Fern Cr at Old Bardstown Road
18 Northern Ditch at Preston Highway
19 Fishpool Cr at Bost Road
20 Southern Ditch at Minors Lane
21 Floyds Fork at Bardstown Road
22 Cedar Cr at Thixton Road
23 Pennsylvania Run at Mt. Washington Road
24 Mill Cr Cutoff at Dover Road
25 Harrods Cr at Hunting Cr Drive
26 Long Run at State Highway 1531

1 Pond $\mathrm{Cr}$ at Pendleton Road

$2 \mathrm{Mill} \mathrm{Cr}$ at Orell Road

3 Pond $\mathrm{Cr}$ at Manslick Road

5 SF Beargrass $\mathrm{Cr}$ at Winter Avenue

6 SF Beargrass $\mathrm{Cr}$ at Trevilian Way

7 MF Beargrass $\mathrm{Cr}$ at Old Cannons Lane

8 MF Beargrass $\mathrm{Cr}$ at Beals Branch Road

9 Spring Ditch at Private Drive

10 Muddy Fork at Mockingbird Valley Road

11 Goose $\mathrm{Cr}$ at U.S. Highway 42

12 Little Goose $\mathrm{Cr}$ at U.S. Highway 42

13 Goose $\mathrm{Cr}$ at Old Westport Road

14 Pope Lick at Pope Lick Road

15 Floyds Fork at former State Highway 155

16 Chenoweth Run at Gelhaus Road

17 Fern $\mathrm{Cr}$ at Old Bardstown Road

18 Northern Ditch at Preston Highway

19 Fishpool $\mathrm{Cr}$ at Bost Road

20 Southern Ditch at Minors Lane

21 Floyds Fork at Bardstown Road

22 Cedar $\mathrm{Cr}$ at Thixton Road

23 Pennsylvania Run at Mt. Washington Road

24 Mill Cr Cutoff at Dover Road

25 Harrods $\mathrm{Cr}$ at Hunting $\mathrm{Cr}$ Drive

26 Long Run at State Highway 1531

1 Pond $\mathrm{Cr}$ at Pendleton Road

$2 \mathrm{Mill} \mathrm{Cr}$ at Orell Road

3 Pond $\mathrm{Cr}$ at Manslick Road

5 SF Beargrass Cr at Winter Avenue

6 SF Beargrass Cr at Trevilian Way

7 MF Beargrass $\mathrm{Cr}$ at Old Cannons Lane

8 MF Beargrass $\mathrm{Cr}$ at Beals Branch Road

9 Spring Ditch at Private Drive

10 Muddy Fork at Mockingbird Valley Road

11 Goose $\mathrm{Cr}$ at U.S. Hi ghway 42

12 Little Goose $\mathrm{Cr}$ at U.S. Highway 42

13 Goose $\mathrm{Cr}$ at Old Westport Road

14 Pope Lick at Pope Lick Road

15 Floyds Fork at former State Highway 155

16 Chenoweth Run at Gelhaus Road

17 Fern $\mathrm{Cr}$ at Old Bardstown Road

18 Northern Ditch at Preston Highway

19 Fishpool $\mathrm{Cr}$ at Bost Road

20 Southern Ditch at Minors Lane

21 Floyds Fork at Bardstown Road

22 Cedar $\mathrm{Cr}$ at Thixton Road

23 Pennsylvania Run at Mt. Washington Road

24 Mill Cr Cutoff at Dover Road

25 Harrods $\mathrm{Cr}$ at Hunting $\mathrm{Cr}$ Drive

26 Long Run at State Highway 1531

1 Pond $\mathrm{Cr}$ at Pendleton Road

$2 \mathrm{Mill} \mathrm{Cr}$ at Orell Road

3 Pond $\mathrm{Cr}$ at Manslick Road

5 SF Beargrass $\mathrm{Cr}$ at Winter Avenue

6 SF Beargrass $\mathrm{Cr}$ at Trevilian Way

$7 \mathrm{MF}$ Beargrass $\mathrm{Cr}$ at Old Cannons Lane

90
87
90
90
90
91
89
91
91

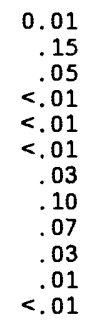

0.04
.35
.38
.29
.27
.24
.10
.27
.18
.16
.06
.02

0.07
.71
.59
.65
.46
.43
.16
.47
.35
.38
.10
.03

0.11
1.2
1.2
1.2
1.0
.77
.28
.87
.87
.60
.20
.06

0.19
1.8
1.8
1.4
1.3
1.1
.52
1.4
1.6
1.1
.45
.14

0.36
2.5
2.4
2.3
1.7
1.4
1.1
1.7
2.0
1.5
.72
.28

1.2

7.5

7.8

5.4
4.7

4.5

4.4

4.9
7.4

3.6

1.7

\section{Arsenic total in $\mu \mathrm{g} / \mathrm{L}$ as As}

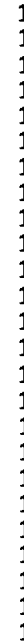

$\begin{array}{ll}18 & 13 \\ 15 & 14 \\ 18 & 13 \\ 16 & 16 \\ 16 & 16 \\ 16 & 16 \\ 16 & 15 \\ 17 & 17 \\ 17 & 16 \\ 16 & 1 \\ 16 & 15 \\ 16 & 15 \\ 18 & 17 \\ 18 & 18 \\ 18 & 18 \\ 17 & 15 \\ 17 & 17 \\ 16 & 12 \\ 16 & 15 \\ 17 & 17 \\ 17 & 17 \\ 17 & 16 \\ 15 & 17 \\ 16 & 15 \\ 12 & 12\end{array}$

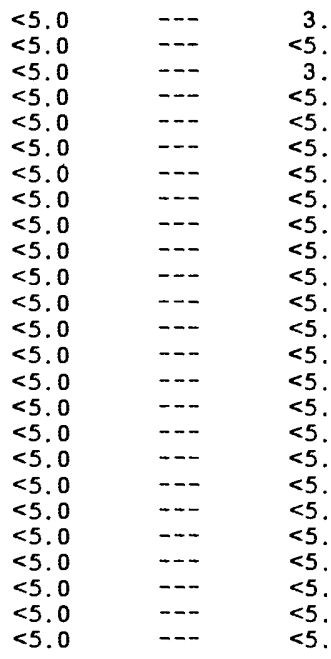

$3.7 *$

$\begin{aligned} & 4.3^{\star} \\ &<5.0 \\ & 4.2^{\star} \\ &<5.0 \\ &<5.0 \\ &<5.0 \\ &<5.0 \\ &<5.0 \\ &<5.0 \\ &<5.0 \\ &<5.0 \\ &<5.0 \\ &<5.0 \\ &<5.0 \\ &<5.0 \\ &<5.0 \\ &<5.0 \\ &<5.0 \\ &<5.0 \\ &<5.0 \\ &<5.0 \\ &<5.0 \\ &<5.0 \\ &<5.0 \\ &<5.0\end{aligned}$

$\begin{aligned} & 5.0 \\ &<5.0 \\ & 5.0 \\ &<5.0 \\ &<5.0 \\ &<5.0 \\ &<5.0 \\ &<5.0 \\ &<5.0 \\ &<5.0 \\ &<5.0 \\ &<5.0 \\ &<5.0 \\ &<5.0 \\ &<5.0 \\ &<5.0 \\ &<5.0 \\ &<5.0 \\ &<5.0 \\ &<5.0 \\ &<5.0 \\ &<5.0 \\ &<5.0 \\ &<5.0 \\ &<5.0\end{aligned}$

\begin{tabular}{cc}
--- & 7.0 \\
--- & 6.0 \\
--- & 7.0 \\
--- & $<5.0$ \\
--- & $<5.0$ \\
--- & $<5.0$ \\
--- & 11 \\
--- & $<5.0$ \\
--- & 9.0 \\
--- & \multicolumn{2}{c}{5.0} \\
--- & 12 \\
--- & 6.0 \\
--- & 22 \\
--- & $<5.0$ \\
--- & $<5.0$ \\
--- & 19 \\
--- & $<10$ \\
--- & 17 \\
--- & 28 \\
--- & $<5.0$ \\
--- & $<5.0$ \\
--- & 10 \\
--- & 13 \\
--- & 7.0 \\
--- & $<5.0$ \\
&
\end{tabular}

Barium, total, in $\mu \mathrm{g} / \mathrm{L}$ as $\mathrm{Ba}$

$\begin{array}{ccccc}18 & 0 & 12 & -- & 40 \\ 15 & 0 & 16 & --- & 38 \\ 18 & 0 & 29 & --- & 42 \\ 16 & 0 & 38 & --- & 48 \\ 16 & 0 & 38 & --- & 54 \\ 16 & 0 & 37 & --- & 44 \\ 16 & 0 & 36 & --- & 43 \\ 16 & 0 & 24 & --- & 38 \\ 17 & 0 & 35 & --- & 44 \\ 16 & 0 & 20 & --- & 37 \\ 16 & 0 & 19 & --- & 40 \\ 16 & 0 & 28 & --- & 36 \\ 18 & 1 & <2.0 & --- & 34 \\ 18 & 0 & 10 & --- & 29 \\ 18 & 0 & 10 & --- & 31 \\ 17 & 0 & 37 & --- & 42 \\ 16 & 0 & 24 & --- & 39 \\ 16 & 0 & 7.0 & --- & 36 \\ 16 & 0 & 26 & --- & 32 \\ 17 & 0 & 29 & --- & 35 \\ 17 & 0 & 20 & --- & 29 \\ 17 & 0 & 30 & --- & 33 \\ 15 & 0 & 16 & --- & 22 \\ 16 & 0 & 9.0 & --- & 39 \\ 12 & 0 & 22 & --- & 24 \\ & & & & \end{array}$

43
44
50
54
57
52
52
42
48
42
47
43
41
38
37
45
40
45
42
42
37
35
25
51
29

$\begin{array}{rr}--- & 60 \\ --- & 68 \\ --- & 66 \\ --- & 87 \\ --- & 73 \\ --- & 67 \\ --- & 70 \\ --- & 53 \\ --- & 86 \\ --- & 64 \\ --- & 86 \\ --- & 85 \\ --- & 56 \\ --- & 60 \\ --- & 110 \\ --- & 628 \\ --- & 64 \\ --- & 54 \\ --- & 56 \\ --- & 57 \\ --- & 57 \\ --- & 72 \\ --- & 32 \\ --- & 157 \\ --- & 177 \\ & \end{array}$

Beryllium, total in $\mu \mathrm{g} / \mathrm{L}$ as $\mathrm{Be}$

$\begin{array}{lllll}17 & 16 & <.20 & -- & <.50 \\ 15 & 14 & <.20 & -- & <.50 \\ 18 & 18 & <.20 & --- & <.40 \\ 16 & 15 & <.20 & --- & <.40 \\ 16 & 15 & <.20 & --- & <.40 \\ 16 & 15 & <.20 & --- & <.50\end{array}$

$<.60$

$<.50$

$<.50$

$<.50$

$<.50$

$<1.0$
$<1.0$
$<1.0$
$<1.0$
$<1.0$
$<1.0$

---
---
---
---
---
---

.60

1.0
$<1.0$

.30

1.7
.60 
Table 13. Statistical summary of constituent concentrations in streams in Jefferson County, Kentucky, 1988-92--Continued

[N, number of observations; $\mathrm{Cr}$, Creek; SF, South Fork; MF, Middle Fork; mg/L, milligrams per liter; ${ }^{\circ} \mathrm{C}$, degrees Celsius; $\mu \mathrm{g} / \mathrm{L}$, micrograms per liter; ---, missing; <, less than; *, value estimated from log-normal fit program; percentiles not computed if fewer than 10 observations; the 10 th and 90th percentiles are not shown if fewer than 30 observations]

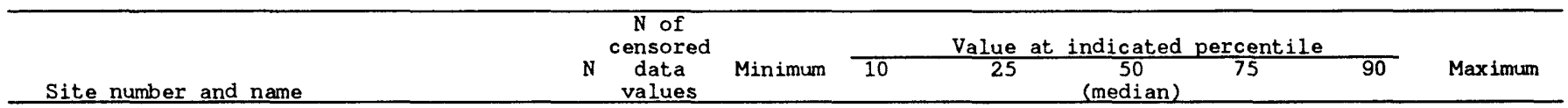

Beryllium, total, in $\mu \mathrm{g} / \mathrm{L}$ as Be--Continued

8 MF Beargrass $\mathrm{Cr}$ at Beals Branch Road 9 Spring Ditch at Private Drive 10 Muddy Fork at Mockingbird Valley Road 11 Goose $\mathrm{Cr}$ at U.S. Highway 42 12 Little Goose $\mathrm{Cr}$ at U.S. Highway 42 13 Goose $\mathrm{Cr}$ at, Old Westport Road 14 Pope Lick at Pope Lick Road 15 Floyds Fork at former State Highway 155 16 Chenoweth Run at Gelhaus Road 17 Fern $\mathrm{Cr}$ at Old Bardstown Road 18 Northern Ditch at Preston Highway 19 Fishpool $\mathrm{Cr}$ at Bost Road 20 Southern Ditch at Minors Lane 21 Floyds Fork at Bardstown Road 22 Cedar $\mathrm{Cr}$ at Thixton Road

23 Pennsylvania Run at Mt. Washington Road 24 Mill Cr Cutoff at Dover Road 25 Harrods $\mathrm{Cr}$ at Hunting $\mathrm{Cr}$ Drive 26 Long Run at State Highway 1531

1 Pond $\mathrm{Cr}$ at Pendleton Road $2 \mathrm{Mill} \mathrm{Cr}$ at Orell Road

3 Pond $\mathrm{Cr}$ at Manslick Road

$5 \mathrm{SF}$ Beargrass $\mathrm{Cr}$ at Winter Avenue

6 SF Beargrass $\mathrm{Cr}$ at Trevilian Way

$7 \mathrm{MF}$ Beargrass $\mathrm{Cr}$ at Old Cannons Lane

8 MF Beargrass $\mathrm{Cr}$ at Beals Branch Road

9 Spring Ditch at Private Drive

10 Muddy Fork at Mockingbird Valley Road

11 Goose $\mathrm{Cr}$ at U.S. Highway 42

12 Little Goose $\mathrm{Cr}$ at U.S. Highway 42

13 Goose $\mathrm{Cr}$ at Old Westport Road

14 Pope Lick at Pope Lick Road

15 Floyds Fork at former State Highway 155

16 Chenoweth Run at Gelhaus Road

17 Fern $\mathrm{Cr}$ at Old Bardstown Road

18 Northern Ditch at Preston Highway

19 Fishpool $\mathrm{Cr}$ at Bost Road

20 Southern Ditch at Minors Lane

21 Floyds Fork at Bardstown Road

22 Cedar $\mathrm{Cr}$ at Thixton Road

23 Pennsylvania Run at Mt. Washington Road

24 Mill Cr Cutoff at Dover Road

25 Harrods $\mathrm{Cr}$ at Hunting $\mathrm{Cr}$ Drive

26 Long Run at State Highway 1531

1 Pond $\mathrm{Cr}$ at Pendleton Road

2 Mill $\mathrm{Cr}$ at Orell Road

3 Pond $\mathrm{Cr}$ at Manslick Road

$5 \mathrm{SF}$ Beargrass $\mathrm{Cr}$ at Winter Avenue

$6 \mathrm{SF}$ Beargrass $\mathrm{Cr}$ at Trevilian Way

$7 \mathrm{MF}$ Beargrass $\mathrm{Cr}$ at Old Cannons Lane

8 MF Beargrass $\mathrm{Cr}$ at Beals Branch Road

9 Spring Ditch at Private Drive

10 Muddy Fork at Mockingbird Valley Road

11 Goose $\mathrm{Cr}$ at U.S. Highway 42

12 Little Goose $\mathrm{Cr}$ at U.S. Highway 42

13 Goose $\mathrm{Cr}$ at Old Westport Road

14 Pope Lick at Pope Lick Road

15 Floyds Fork at former State Highway 155

16 Chenoweth Run at Gelhaus Road

17 Fern $\mathrm{Cr}$ at Old Bardstown Road

18 Northern Ditch at Preston Highway

$19 \mathrm{Fishpool} \mathrm{Cr}$ at Bost Road

20 Southern Ditch at Minors Lane

21 Floyds Fork at Bardstown Road

22 Cedar $\mathrm{Cr}$ at Thixton Road

23 Pennsylvania Run at Mt. Washington Road

24 Mill Cr Cutoff at Dover Road

25 Harrods $\mathrm{Cr}$ at Hunting $\mathrm{Cr}$ Drive

26 Long Run at State Highway 1531

$\begin{array}{ll}16 & 15 \\ 16 & 13 \\ 17 & 17 \\ 10 & \\ 12 & \\ 16 & 15 \\ 11 & \\ 18 & 18 \\ 18 & 17 \\ 17 & 14 \\ 12 & 10 \\ 16 & 16 \\ 12 & 10 \\ 17 & 16 \\ 17 & 16 \\ 12 & 10 \\ 12 & 10 \\ 16 & 12 \\ 12 & 1\end{array}$

$<0.20$

$<.20$
$<.20$

$<.20$
$<.20$

$<.20$
$<.20$

$<.20$

$<.20$

$<.20$

$<.20$

$<.20$

$<.20$

$<.20$

$<.20$

$<.20$

$<.20$

$<.20$
$<.20$
$<.20$

$<.20$

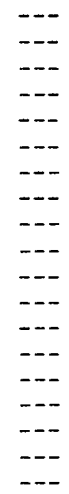

$<0.50$
$.03^{*}$

$.03^{*}$
$<.50$

$<.40$

$.14 *$

$<.40$

$.11^{*}$

$<.50$
$<.50$

$.03 *$

$<.50$

$<.50$
$<.40$

$<.20$

$<.50$

$<.50$

$<.20$
$<.20$

$.09^{*}$
$<.50$

$<0.50$
$.10 *$

$.10^{*}$
$<.50$

$<.50$

$.22 *$

$<.50$

$<.19^{*}$

$<.50$
$<.60$

$.09^{*}$

$<.50$

$<.50$

$<.50$
$<.50$

$<.50$

$<.50$

$<.50$

$.20 *$

$.20^{*}$
$<.50$

$<1.0$

$.33^{*}$
$<.90$

$<.60$

$.36 *$

$<1.0$

$.33^{*}$
$<1.0$

$<1.0$

$.28^{*}$

$.28 *$
$<.50$

$<.90$

$<.60$

$<1.0$

$<1.0$

$<.50$
$<.90$

.44 *

Cadmium, total, in $\mu \mathrm{g} / \mathrm{L}$ as $\mathrm{Cd}$

\begin{tabular}{|c|c|c|c|c|}
\hline 18 & 17 & $<2.0$ & --- & $<4$ \\
\hline 15 & 14 & $<2.0$ & --- & $<4$ \\
\hline 18 & 16 & $<2.0$ & $-\cdots$ & $<5$ \\
\hline 16 & 16 & $<2.0$ & $\cdots$ & $<4$ \\
\hline 16 & 16 & $<2.0$ & -- & $<4$ \\
\hline 16 & 16 & $<2.0$ & -- & $<4$ \\
\hline 16 & 15 & $<2.0$ & -- & $<4$ \\
\hline 16 & 16 & $<2.0$ & -- & $<4$ \\
\hline 17 & 17 & $<2.0$ & -- & $<4$ \\
\hline 16 & 16 & $<2.0$ & --- & $<4$ \\
\hline 16 & 15 & $<2.0$ & -- & $<4$ \\
\hline 10 & 9 & $<1.0$ & -- & $<4$ \\
\hline 18 & 18 & $<2.0$ & --- & $<4$ \\
\hline 18 & 16 & $<2.0$ & $-\cdots$ & $<6$ \\
\hline & 16 & $<2.0$ & -- & $<4$ \\
\hline 15 & 13 & $<2.0$ & --- & $<4$ \\
\hline & 15 & $<2.0$ & --- & $<4$ \\
\hline & 15 & $<2.0$ & $\cdots$ & $<4$ \\
\hline & 16 & $<2.0$ & --- & $<4$ \\
\hline 17 & 16 & $<2.0$ & $-\cdots$ & $<6$ \\
\hline 17 & 17 & $<2.0$ & -- & $<4$ \\
\hline 13 & 11 & $<4.0$ & $z^{--}$ & $<4$ \\
\hline 15 & 14 & $<2.0$ & $=-$ & \\
\hline & 15 & $<2.0$ & --- & $<4$ \\
\hline & 12 & $<3.0$ & - - & $<$ \\
\hline
\end{tabular}

Chromium, total, in $\mu \mathrm{g} / \mathrm{L}$ as $\mathrm{Cr}$

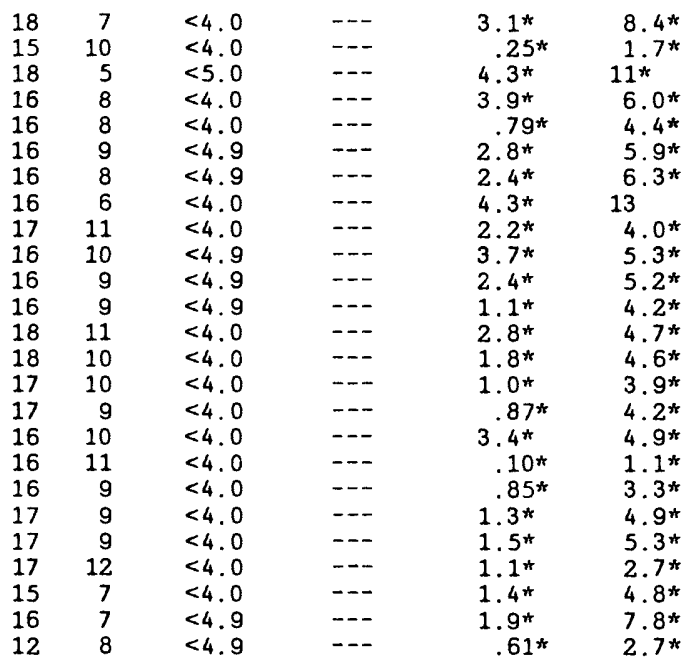

$<9.0$

$<9.0$

$<9.0$

$<6.9$

$<6.4$

$<6.4$

$<6.4$

$<6.4$

$<6.9$

$<6.9$

$<9.0$

$<9.0$

$<9.0$

$<9.0$

$<9.0$

$<6.4$

$<6.9$

$<6.4$

$<6.4$

$<9.0$

$<9.0$

$<6.4$

$<9.0$

$<9.0$

$<9.0$
0.20

3.5

$<1.0$

1.0

.60

$<1.0$

3.4

.90

$<1.0$

1.0

.90

1.0

1.3

3.0
10
11
$<9.0$
$<9.0$
$<9.0$
16
$<9.0$
$<9.0$
$<9.0$
4.0
6.0
$<9.0$
19
11
9.0
3.0
15
$<9.0$
2.0
$<9.0$
7.0
13
2.0
$<9.0$

$\begin{array}{llr}13 & --- & 520 \\ 11 & --- & 700 \\ 20 & --- & 510 \\ 12 & --- & 13 \\ 13 & --- & 1,210 \\ 19 & --- & 34 \\ 15 & --- & 142 \\ 20 & --- & 44 \\ 7.3^{*} & --- & 17 \\ 7.7^{*} & --- & 15 \\ 11^{*} & --- & 79 \\ 15 & --- & 520 \\ 7.8^{*} & --- & 21 \\ 14 & --- & 139 \\ 14^{*} & --- & 337 \\ 16 & --- & 1,200 \\ 7.2^{*} & --- & 12 \\ 12^{*} & --- & 670 \\ 13^{*} & --- & 690 \\ 16 & --- & 510 \\ 17 & --- & 530 \\ 7.0^{*} & --- & 29 \\ 16^{*} & --- & 684 \\ 24 & --- & 1,210 \\ 12^{*} & --- & 189\end{array}$


Table 13. Statistical summary of constituent concentrations in streams in Jefferson County, Kentucky, 1988-92--Continued

[N, number of observations; Cr, Creek; SF, South Fork; MF, Middle Fork; mg/L, milligrams per 1 iter; ${ }^{\circ} \mathrm{C}$, degrees Celsius; $\mu_{8} / L$, micrograms per liter; --., missing; <, less than; *, value estimated from log-normal fit program; percentiles not computed if fewer than 10 observations; the 10 th and 90th percentiles are not shown if fewer than 30 observations]

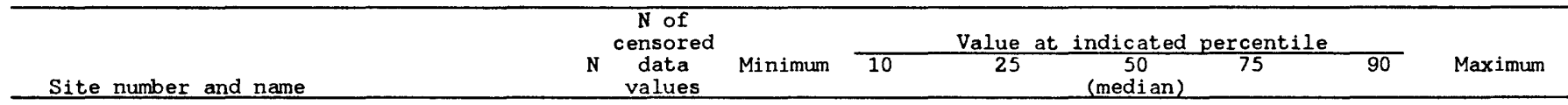

1 Pond $\mathrm{Cr}$ at Pendleton Road

$2 \mathrm{Mill} \mathrm{Cr}$ at Orell Road

3 Pond $\mathrm{Cr}$ at Manslick Road

$5 \mathrm{SF}$ Beargrass $\mathrm{Cr}$ at Winter Avenue

$6 \mathrm{SF}$ Beargrass $\mathrm{Cr}$ at Trevilian Way

$7 \mathrm{MF}$ Beargrass $\mathrm{Cr}$ at Old Cannons Lane

$8 \mathrm{MF}$ Beargrass $\mathrm{Cr}$ at Beals Branch Road

9 Spring Ditch at Private Drive

10 Muddy Fork at Mockingbird Valley Road

11 Goose $\mathrm{Cr}$ at U.S. Highway 42

12 Little Goose $\mathrm{Cr}$ at U.S. Highway 42

13 Goose $\mathrm{Cr}$ at Old Westport Road

14 Pope Lick at Pope Lick Road

15 Floyds Fork at former State Highway 155

16 Chenoweth Run at Gelhaus Road

17 Fern $\mathrm{Cr}$ at Old Bardstown Road

18 Northern Ditch at Preston Highway

19 Fishpool $\mathrm{Cr}$ at Bost Road

20 Southern Ditch at Minors Lane

21 Floyds Fork at Bardstown Road

22 Cedar $\mathrm{Cr}$ at Thixton Road

23 Pennsylvania Run at Mt. Washington Road

$24 \mathrm{Mill} \mathrm{Cr}$ Cutoff at Dover Road

25 Harrods $\mathrm{Cr}$ at Hunting $\mathrm{Cr}$ Drive

26 Long Run at State Highway 1531

1 Pond $\mathrm{Cr}$ at Pendleton Road

2 Mill $\mathrm{Cr}$ at Orell Road

3 Pond $\mathrm{Cr}$ at Manslick Road

$5 \mathrm{SF}$ Beargrass $\mathrm{Cr}$ at Winter Avenue

6 SF Beargrass $\mathrm{Cr}$ at Trevilian Way

7 MF Beargrass $\mathrm{Cr}$ at Old Cannons Lane

$8 \mathrm{MF}$ Beargrass $\mathrm{Cr}$ at Beals Branch Road

9 Spring Ditch at Private Drive

10 Muddy Fork at Mockingbird Valley Road

11 Goose $\mathrm{Cr}$ at U.S. Highway 42

12 Little Goose $\mathrm{Cr}$ at U.S. Highway 42

13 Goose $\mathrm{Cr}$ at Old Westport Road

14 Pope Lick at Pope Lick Road

15 Floyds Fork at former State Highway 155

16 Chenoweth Run at Gelhaus Road

17 Fern Cr at Old Bardstown Road

18 Northern Ditch at Preston Highway

19 Fishpool $\mathrm{Cr}$ at Bost Road

20 Southern Ditch at Minors Lane

21 Floyds Fork at Bardstown Road

22 Cedar $\mathrm{Cr}$ at Thixtion Road

23 Pennsylvania Run at Mt. Washington Road

$24 \mathrm{Mill}$ Cr Cutoff at Dover Road

25 Harrods $\mathrm{Cr}$ at Hunting $\mathrm{Cr}$ Drive

26 Long Run at State Highway 1531

1 Pond $\mathrm{Cr}$ at Pendleton Road

$2 \mathrm{Mill} \mathrm{Cr}$ at Orell Road

3 Pond $\mathrm{Cr}$ at Manslick Road

$5 \mathrm{SF}$ Beargrass $\mathrm{Cr}$ at Winter Avenue

6 SF Beargrass $\mathrm{Cr}$ at Trevilian Way

7 MF Beargrass $\mathrm{Cr}$ at Old Cannons Lane

9 Spring Ditch at Private Drive

10 Muddy Fork at Mockingbird Valley Road

11 Goose $\mathrm{Cr}$ at U.S. Highway 42

12 Little Goose $\mathrm{Cr}$ at U.S. Highway 42

13 Goose $\mathrm{Cr}$ at Old Westport Road

14 Pope Lick at Pope Lick Road

15 Floyds Fork at former State Highway 155

16 Chenoweth Run at Gelhaus Road

17 Fern $\mathrm{Cr}$ at Old Bardstown Road

18 Northern Ditch at Preston Highway

19 Fishpool $\mathrm{Cr}$ at Bost Road

20 Southern Ditch at Minors Lane

21 Floyds Fork at Bardstown Road

22 Cedar $\mathrm{Cr}$ at Thixton Road

Copper, total recoverable, in $\mu g / \mathrm{L}$ as $\mathrm{Cu}$

\begin{tabular}{|c|c|c|c|c|}
\hline 18 & 8 & $<6.0$ & --- & $3.5^{*}$ \\
\hline 15 & 8 & $<6.0$ & --- & $3.0 *$ \\
\hline 18 & 8 & $<6.0$ & $\cdots$ & $3.6^{*}$ \\
\hline 16 & 6 & $<6.0$ & -- & $5.0 *$ \\
\hline 16 & 8 & $<6.0$ & -- & $3.1^{*}$ \\
\hline 16 & 8 & $<5.0$ & $\cdots$ & $2.3^{*}$ \\
\hline 16 & 5 & $<6.0$ & $\cdots$ & $5.0 *$ \\
\hline 16 & 3 & $<8.0$ & $\cdots$ & $8.1^{*}$ \\
\hline 17 & 5 & $<5.0$ & $-\cdots$ & $3.0^{*}$ \\
\hline 16 & 5 & $<6.0$ & --- & $5.6^{*}$ \\
\hline 16 & 8 & $<6.0$ & $-\cdots$ & $3.4 *$ \\
\hline 16 & 4 & $<6.0$ & -- & $6.6^{*}$ \\
\hline 18 & 4 & $<6.0$ & -- & $6.4^{*}$ \\
\hline 18 & 6 & $<6.0$ & --- & $5.8^{*}$ \\
\hline 18 & 8 & $<6.0$ & --- & $3.8^{*}$ \\
\hline 17 & 6 & $<6.0$ & $\cdots$ & $4.5^{*}$ \\
\hline 16 & 4 & $<6.0$ & -- & $8.0 *$ \\
\hline 16 & 8 & $<6.0$ & $\cdots$ & $2.6^{*}$ \\
\hline 16 & 6 & $<6.0$ & $\cdots$ & $5.0 *$ \\
\hline 17 & 5 & $<6.0$ & -- & $6.1^{*}$ \\
\hline 17 & 6 & $<5.0$ & --- & $4.9 *$ \\
\hline 17 & 5 & $<5.0$ & $\cdots$ & $4.9^{*}$ \\
\hline 15 & 7 & $<6.0$ & --- & $3.5^{*}$ \\
\hline 16 & 8 & $<5.0$ & $\cdots$ & $2.6^{*}$ \\
\hline 12 & 3 & $<6.0$ & $\cdots$ & $7.9 *$ \\
\hline
\end{tabular}

8.5
$6.5^{\star}$
11.5
8.5
$7.1^{*}$
$7.7^{*}$
9.0
14
10
11
$7.0 *$
12
11
11
$9.2 *$
10
13
$7.4 *$
$11 *$
9.0
9.0
9.0
8.0
$7.0 *$
15

25
15
36
22
$16^{*}$
36
29
28
20
29
20
31
20
22
31
30
23
20
23
15
19
14
15
24
31

$\begin{array}{rr}--- & 96 \\ --- & 55 \\ --- & 77 \\ --- & 37 \\ --- & 73 \\ --- & 146 \\ --- & 130 \\ --- & 51 \\ --- & 1,820 \\ --- & 73 \\ --- & 40 \\ --- & 62 \\ --- & 56 \\ --- & 57 \\ --- & 88 \\ --- & 87 \\ --- & 50 \\ --- & 103 \\ --- & 130 \\ --- & 40 \\ --- & 37 \\ --- & 82 \\ --- & 74 \\ --- & 120 \\ --- & 43\end{array}$

\section{Iron, total, in $\mu \mathrm{g} / \mathrm{L}$ as $\mathrm{Fe}$}

18
15
18
16
16
16
16
16
17
16
16
16
18
18
18
17
16
16
16
17
17
17
15
16
12

$\begin{array}{rrr}<10 & -- & 265 \\ 209 & -- & 263 \\ 370 & --- & 789 \\ 160 & --- & 475 \\ <10 & --- & 213 \\ <10 & --- & 173 \\ <10 & --- & 189 \\ 50 & --- & 244 \\ <40 & -- & 253 \\ <10 & --- & 78 \\ 180 & --- & 229 \\ <10 & --- & 164 \\ <10 & --- & 164 \\ 10 & --- & 255 \\ <10 & --- & 106 \\ <10 & --- & 119 \\ 90 & --- & 168 \\ <10 & --- & 214 \\ 240 & --- & 415 \\ <10 & --- & 238 \\ <10 & --- & 120 \\ <10 & --- & 223 \\ 102 & --- & 250 \\ <10 & --- & 482 \\ 149 & --- & 249\end{array}$

707

1,110

506

252

602

416

580
229

229
477

473

267

744

284

268

225

268

687

520

190

520
595

595
748

680

Lead, total, in $\mu \mathrm{g} / \mathrm{L}$ as $\mathrm{Pb}$

$\begin{array}{lllll}18 & 15 & <40 & --- & 22^{*} \\ 15 & 13 & <10 & --- & <40 \\ 18 & 17 & <40 & -- & <40 \\ 16 & 15 & <40 & --- & <40 \\ 16 & 16 & <40 & --- & <40 \\ 15 & 13 & <10 & --- & <40 \\ 13 & 10 & <40 & --- & 29^{*} \\ 17 & 17 & <20 & -- & <40 \\ 16 & 16 & <40 & -- & <40 \\ 16 & 12 & <10 & --- & 5.9 * \\ 16 & 15 & <40 & --- & <40 \\ 16 & 14 & <40 & --- & <40 \\ 17 & 15 & <40 & --- & <40 \\ 18 & 17 & <40 & --- & <40 \\ 17 & 15 & <10 & --- & <40 \\ 16 & 16 & <40 & --- & <40 \\ 16 & 15 & <40 & --- & <40 \\ 16 & 16 & <40 & --- & <40 \\ 17 & 17 & <10 & --- & <40 \\ 17 & 16 & <40 & -- & <40\end{array}$

$\begin{array}{rrr}1,210 & --- & 16,900 \\ 2,830 & -- & 13,700 \\ 2,650 & -- & 3,530 \\ 1,840 & --- & 10,800 \\ 1,170 & --- & 6,120 \\ 900 & --- & 2,720 \\ 1,110 & --- & 4,660 \\ 1,440 & -- & 10,600 \\ 1,080 & --- & 3,580 \\ 565 & --- & 1,760 \\ 1,600 & --- & 5,210 \\ 1,110 & --- & 2,680 \\ 1,150 & -- & 17,700 \\ 2,110 & --- & 19,500 \\ 580 & --- & 7,700 \\ 775 & --- & 69,200 \\ 691 & --- & 2,850 \\ 391 & --- & 2,810 \\ 855 & --- & 1,720 \\ 935 & --- & 5,530 \\ 370 & --- & 2,200 \\ 895 & --- & 18,100 \\ 1,460 & --- & 1,930 \\ 1,610 & --- & 14,100 \\ 1,800 & --- & 3,080\end{array}$

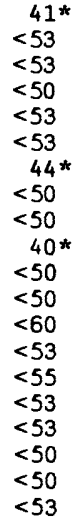

$\begin{array}{rr}--- & 70 \\ --- & 170 \\ --- & 190 \\ -- & <50 \\ -- & <60 \\ -- & 60 \\ --- & 60 \\ -- & <60 \\ -- & <60 \\ -- & 150 \\ -- & 70 \\ -- & 60 \\ -- & 100 \\ -- & 70 \\ -- & 120 \\ -- & <60 \\ -- & 100 \\ -- & <60 \\ -- & <60 \\ -- & 80\end{array}$ 


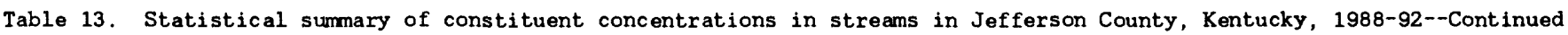

IN, number of observations; $\mathrm{Cr}$, Creek; SF, South Fork; MF, Middle Fork; mg/L, milligrams per 1iter; ${ }^{\circ} \mathrm{C}$, degrees Celsius; $\mu \mathrm{g} / \mathrm{L}$, micrograms per liter; ---, missing; <, less than; *, value estimated from log-normal fit program; percentiles not computed if fewer than 10 observations; the 10 th and 90 th percentiles are not shown if fewer than 30 observations]

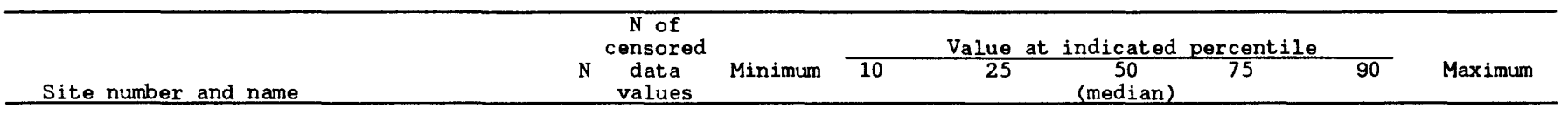

Lead, total, in $\mu \mathrm{B} / \mathrm{L}$ as $\mathrm{Pb}--$ Continued

23 Pennsylvania Run at Mt. Washington Road $24 \mathrm{Mill} \mathrm{Cr}$ Cutoff at Dover Road 25 Harrods $\mathrm{Cr}$ at Hunting $\mathrm{Cr}$ Drive 26 Long Run at State Highway 1531

$\begin{array}{ll}17 & 16 \\ 15 & 13 \\ 16 & 16 \\ 12 & 11\end{array}$

$<40$
$<40$
$<40$
$<40$

$$
\begin{aligned}
& <50 \\
& <40 \\
& <40 \\
& <40
\end{aligned}
$$$$
\begin{aligned}
& <50 \\
& <50 \\
& <50 \\
& <50
\end{aligned}
$$$$
\begin{aligned}
& <55 \\
& <55 \\
& <53 \\
& <60
\end{aligned}
$$$$
\begin{array}{rr}
-- & 50 \\
-- & 170 \\
--- & <60 \\
-- & 130
\end{array}
$$

Mercury, total recoverable, in $\mu \mathrm{g} / \mathrm{L}$ as $\mathrm{Hg}$

1 Pond $\mathrm{Cr}$ at Pendleton Road $2 \mathrm{Mill} \mathrm{Cr}$ at Orell Road

3 Pond $\mathrm{Cr}$ at Manslick Road

5 SF Beargrass $\mathrm{Cr}$ at Winter Avenue

6 SF Beargrass $\mathrm{Cr}$ at Trevilian Way

7 MF Beargrass $\mathrm{Cr}$ at Old Cannons Lane

8 MF Beargrass $\mathrm{Cr}$ at Beals Branch Road

9 Spring Ditch at Private Drive

10 Muddy Fork at Mockingbird Valley Road

11 Goose $\mathrm{Cr}$ at U.S. Highway 42

12 Little Goose $\mathrm{Cr}$ at U.S. Highway 42

13 Goose $\mathrm{Cr}$ at Old Westport Road

14 Pope Lick at Pope Lick Road

15 Floyds Fork at former State Highway 155

16 Chenoweth Run at Gelhaus Road

17 Fern $\mathrm{Cr}$ at Old Bardstown Road

18 Northern Ditch at Preston Highway

$19 \mathrm{Fishpool} \mathrm{Cr}$ at Bost Road

20 Southern Ditch at Minors Lane

21 Floyds Fork at Bardstown Road

22 Cedar $\mathrm{Cr}$ at Thixton Road

23 Pennsylvania Run at Mt. Washington Road

$24 \mathrm{Mill} \mathrm{Cr}$ Cutoff at Dover Road

25 Harrods $\mathrm{Cr}$ at Hunting $\mathrm{Cr}$ Drive

26 Long Run at State Highway 1531

1 Pond $\mathrm{Cr}$ at Pendleton Road

$2 \mathrm{Mill} \mathrm{Cr}$ at Orell Road

3 Pond $\mathrm{Cr}$ at Manslick Road

5 SF Beargrass $\mathrm{Cr}$ at Winter Avenue

6 SF Beargrass $\mathrm{Cr}$ at Trevilian Way

7 MF Beargrass $\mathrm{Cr}$ at Old Cannons Lane

$8 \mathrm{MF}$ Beargrass $\mathrm{Cr}$ at Beals Branch Road

9 Spring Ditch at Private Drive

10 Muddy Fork at Mockingbird Valley Road

11 Goose $\mathrm{Cr}$ at U.S. Highway 42

12 Little Goose $\mathrm{Cr}$ at U.S. Highway 42

13 Goose $\mathrm{Cr}$ at Old Westport Road

14 Pope Lick at Pope Lick Road

15 Floyds Fork at former State Highway 155

16 Chenoweth Run at Gelhaus Road

17 Fern $\mathrm{Cr}$ at Old Bardstown Road

18 Northern Ditch at Preston Highway

$19 \mathrm{Fishpool} \mathrm{Cr}$ at Bost Road

20 Southern Ditch at Minors Lane

21 Floyds Fork at Bardstown Road

22 Cedar $\mathrm{Cr}$ at Thixton Road

23 Pennsylvania Run at Mt. Washington Road

$24 \mathrm{Mill} \mathrm{Cr}$ Cutoff at Dover Road

25 Harrods $\mathrm{Cr}$ at Hunting $\mathrm{Cr}$ Drive

26 Long Run at State Highway 1531

1 Pond $\mathrm{Cr}$ at Pendleton Road

$2 \mathrm{Mill} \mathrm{Cr}$ at Orell Road

3 Pond $\mathrm{Cr}$ at Manslick Road

5 SF Beargrass $\mathrm{Cr}$ at Winter Avenue

6 SF Beargrass $\mathrm{Cr}$ at Trevilian Way

7 MF Beargrass $\mathrm{Cr}$ at Old Cannons Lane

8 MF Beargrass $\mathrm{Cr}$ at Beals Branch Road

9 Spring Ditch at Private Drive

10 Muddy Fork at Mockingbird Valley Road

11 Goose $\mathrm{Cr}$ at U.S. Highway 42

12 Little Goose $\mathrm{Cr}$ at U.S. Highway 42

13 Goose $\mathrm{Cr}$ at Old Westport Road

14 Pope Lick at Pope Lick Road

15 Floyds Fork at former State Highway 155

$\begin{array}{lr}17 & 12 \\ 15 & 11 \\ 17 & 12 \\ 16 & 10 \\ 16 & 9 \\ 16 & 9 \\ 16 & 12 \\ 17 & 10 \\ 17 & 9 \\ 16 & 12 \\ 16 & 11 \\ 13 & 8 \\ 17 & 9 \\ 17 & 9 \\ 18 & 12 \\ 16 & 9 \\ 17 & 12 \\ 15 & 10 \\ 15 & 9 \\ 17 & 7 \\ 17 & 7 \\ 17 & \\ 15 & 11 \\ 15 & 10 \\ 12 & 11 \\ & \end{array}$

$<.20 \quad--<20$

$<.20<-.-\quad<.01 *$

$<.20$

$<.20$

$<.20$

$--$

$<.20$

$<.20$

$<.20$

$<.20$

$<.20$

$<.20$

$<.20$

$<.20$

$<.20$

$<.20$

$<.20$

$<.20$

$<.20$

$<.20$

$<.20$

$<.20$
$<.20$

$<.20$

$<.20$

$<.01 *$

$.05 *$

$\ldots$

$--$

$.01^{\star}$

$.11^{*}$

$--$

$-$

$--$

$--$

$--$

$-\cdots$

$--$

$\cdots$

--

$--$

-.-

$<.20$
$<.20$

$-$

$.05^{*}$

$.12^{*}$

$<.20$

$<.20$
$.07 *$

$.09^{*}$

$.10^{*}$

$.03^{*}$

$.08^{\star}$

$.09 *$

$.10 *$

$.10^{*}$

$10^{*}$
$.10^{*}$

$.05^{*}$

$<.06^{*}$

\section{Nickel, total, in $\mu \mathrm{g} / \mathrm{L}$ as $\mathrm{Ni}$}

$\begin{array}{ll}18 & 12\end{array}$

$15 \quad 12$

$18 \quad 11$

$\begin{array}{rr}12 & 12 \\ 10 & 2\end{array}$

$\begin{array}{ll}10 & 2 \\ 16 & 13\end{array}$

$16 \quad 13$

$\begin{array}{rr}16 & 6 \\ 17 & 13\end{array}$

$\begin{array}{ll}17 & 13 \\ 16 & 12\end{array}$

$16 \quad 11$

$18 \quad 14$

$18 \quad 14$

1814

$17 \quad 13$

$16 \quad 12$

1111

$16 \quad 10$

$17 \quad 14$

$\begin{array}{rr}11 & 8 \\ 17 & 13\end{array}$

$15 \quad 12$

$16 \quad 13$

$<7.0$

$<7.0$
$<7.0$

$<6.7$

$<10$

$<6.7$

$<6.7$

$<7.0$

$<6.7$

$<7.0$

$<7.0$

$<6.7$

$<6.0$

$<7.0$

$<7.0$

$<6.7$

$<6.7$

$<6.7$

$<7.0$

$<7.0$

$<7.0$

$<7.0$

$<6.7$

-.-

$--$

-

-.-

$--$

$--$

$--$

$--$

-

- -

$--$

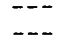

$--$

-..

$--$

$--$

--

$--$

$--$

$<7.0$

--

5. 8 *

3. $1^{*}$

4
$<10$

8.5

$.42^{\star}$

$2.5 *$

$8.1 *$

$2.0 *$

$2.5 *$

$<10$

10

1. 8*

$2.0^{*}$

2. $0 *$

1. $2^{\star}$

$<10$

3. $2^{*}$

$1.8^{*}$

$3.6^{\text {th }}$

1. $6 *$

2.4 *

$2.4^{*}$

Silver, total, in $\mu \mathrm{g} / \mathrm{L}$ as $\mathrm{Ag}$

$\begin{array}{rrr}14 & 11 & <1.0 \\ 15 & 12 & <1.0 \\ 18 & 17 & <1.0 \\ 16 & 16 & <1.0 \\ 16 & 14 & <1.0 \\ 14 & 11 & <1.0 \\ 10 & 8 & <1.0 \\ 11 & 9 & <1.0 \\ 17 & 16 & <1.0 \\ 16 & 14 & <1.0 \\ 11 & 9 & <1.0 \\ 16 & 12 & <1.0 \\ 17 & 11 & <1.0 \\ 16 & 14 & <1.0\end{array}$

בי-

$.50^{\star}$
$.22^{\star}$
$<1.0$
$<1.0$
$<1.0$
$.26^{\star}$
$<1.0$
$<1.0$
$<1.0$
$<1.0$
$<1.0$
$.05^{\star}$
$.91^{\star}$
$<1.0$

1. 0 *

$.75 *$

$<4.7$
$<4.0$

$<4.7$

$.68^{\star}$

$<2.0$

$<4.0$
$<4.0$

$<4.0$

$<4.0$

$.35^{*}$

$<4.7^{*}$

$<1.0$

.20

$.07 *$

.30
.30

.37

.40

.21 *

.25

$.20 *$

.20

.25

.34 *

.30

.55
.25

.30

$.25 *$

.65
.45

.55

.20

30
+30

$<.20$

בי-

.30

1.90

3.1

.50

1.6

.60

.70

1. 1

1.5

1.5

6. 0

1.0
60

.90

4.0

5.2
1.0

1.0

1.0

.90

\begin{tabular}{|c|c|}
\hline $15^{*}$ & $\cdots$ \\
\hline $8.9 \star$ & -- \\
\hline $19 *$ & $\cdots$ \\
\hline$<20$ & -- \\
\hline $12^{\star}$ & -- \\
\hline $6.7^{\star}$ &.-- \\
\hline $8.1^{\star}$ & -- \\
\hline $20 *$ & -.. \\
\hline $9.4 *$ & -- \\
\hline $11^{*}$ & -- \\
\hline $11 *$ & $\cdots$ \\
\hline$<20$ & -.- \\
\hline $9.3 *$ & $\cdots$ \\
\hline $13^{*}$ & $\cdots$ \\
\hline $9.8^{*}$ & -- \\
\hline $9.5^{\star}$ & -- \\
\hline $9.7^{\star}$ & - \\
\hline$<20$ & --- \\
\hline $14^{*}$ & $\cdots$ \\
\hline $8.3^{*}$ & -- \\
\hline $8.4 *$ & --- \\
\hline $9.2^{\star}$ & \\
\hline $9.7 \star$ & $\cdots$ \\
\hline $8.4 *$ & --- \\
\hline $11 *$ & \\
\hline
\end{tabular}

31
25
89
13
16
99
22
39
50
47
23
13
49
61
30
81
100
10
44
28
20
50
30
30
31 
Table 13. Statistical summary of constituent concentrations in streams in Jefferson County, Kentucky, 1988-92--Continued

IN, number of observations; Cr, Creek; SF, South Fork; MF, Middle Fork; mg/L, milligrams per 1iter; ${ }^{\circ} \mathrm{C}$, degrees Celsius; $\mu \mathrm{g} / \mathrm{L}$, micrograms per liter; ---, missing; <, less than; *, value estimated from $\log$-normal fit program; percentiles not computed if fewer than 10 observations; the 10 th and 90 th percentiles are not shown if fewer than 30 observations]

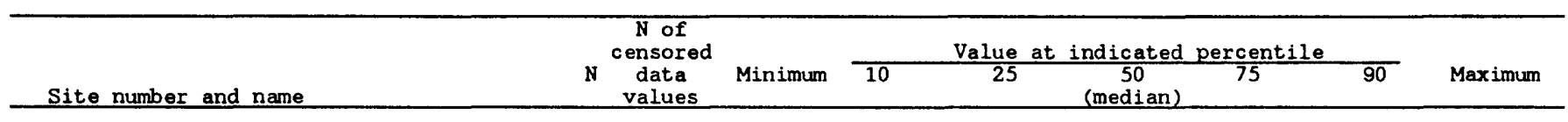

Silver, total, in $\mu \mathrm{g} / \mathrm{L}$ as $\mathrm{Ag}-$-Continued

\author{
16 Chenoweth Run at Gelhaus Road \\ 17 Fern $\mathrm{Cr}$ at Old Bardstown Road \\ 18 Northern Ditch at Preston Highway \\ $19 \mathrm{Fishpool} \mathrm{Cr}$ at Bost Road \\ 20 Southern Ditch at Minors Lane \\ 21 Floyds Fork at Bardstown Road \\ 22 Cedar $\mathrm{Cr}$ at Thixton Road \\ 23 Pennsylvania Run at Mt. Washington Road \\ $24 \mathrm{M} 111 \mathrm{Cr}$ Cutoff at Dover Road \\ 25 Harrods $\mathrm{Cr}$ at Hunting Cr Drive \\ 26 Long Run at State Highway 1531
}

1 Pond $\mathrm{Cr}$ at Pendleton Road

$2 \mathrm{Mill} \mathrm{Cr}$ at Orell Road

3 Pond $\mathrm{Cr}$ at Manslick Road

5 SF Beargrass $\mathrm{Cr}$ at Winter Avenue

6 SF Beargrass $\mathrm{Cr}$ at Trevilian Way

7 MF Beargrass $\mathrm{Cr}$ at Old Cannons Lane

8 MF Beargrass $\mathrm{Cr}$ at Beals Branch Road

9 Spring Ditch at Private Drive

10 Muddy Fork at Mockingbird Valley Road

11 Goose $\mathrm{Cr}$ at U.S. Highway 42

12 Little Goose $\mathrm{Cr}$ at U.S. Highway 42

13 Goose $\mathrm{Cr}$ at Old Westport Road

14 Pope Lick at Pope Lick Road

15 Floyds Fork at former State Highway 155

16 Chenoweth Run at Gelhaus Road

17 Fern $\mathrm{Cr}$ at Old Bardstown Road

18 Northern Ditch at Preston Highway

19 Fishpool $\mathrm{Cr}$ at Bost Road

20 Southern Ditch at Minors Lane

21 Floyds Fork at Bardstown Road

22 Cedar $\mathrm{Cr}$ at Thixton Road

23 Pennsylvania Run at Mt. Washington Road

$24 \mathrm{Mill} \mathrm{Cr}$ Cutoff at Dover Road

25 Harrods $\mathrm{Cr}$ at Hunting $\mathrm{Cr}$ Drive

26 Long Run at State Highway 1531

1 Pond $\mathrm{Cr}$ at Pendleton Road

$2 \mathrm{Mill} \mathrm{Cr}$ at Orell Road

3 Pond $\mathrm{Cr}$ at Manslick Road

5 SF Beargrass $\mathrm{Cr}$ at Winter Avenue

6 SF Beargrass $\mathrm{Cr}$ at Trevilian Way

7 MF Beargrass $\mathrm{Cr}$ at Old Cannons Lane

$8 \mathrm{MF}$ Beargrass $\mathrm{Cr}$ at Beals Branch Road

9 Spring Ditch at Private Drive

10 Muddy Fork at Mockingbird Valley Road

11 Goose $\mathrm{Cr}$ at U.S. Highway 42

12 Little Goose $\mathrm{Cr}$ at U.S. Highway 42

13 Goose $\mathrm{Cr}$ at Old Westport Road

14 Pope Lick at Pope Lick Road

15 Floyds Fork at former State Highway 155

16 Chenoweth Run at Gelhaus Road

17 Fern Cr at Old Bardstown Road

18 Northern Ditch at Preston Highway

$19 \mathrm{Fishpool} \mathrm{Cr}$ at Bost Road

20 Southern Ditch at Minors Lane

21 Floyds Fork at Bardstown Road

22 Cedar $\mathrm{Cr}$ at Thixton Road

23 Pennsylvania Run at Mt. Washington Road

$24 \mathrm{Mill} \mathrm{Cr}$ Cutoff at Dover Road

25 Harrods $\mathrm{Cr}$ at Hunting $\mathrm{Cr}$ Drive

26 Long Run at State Highway 1531

1 Pond $\mathrm{Cr}$ at Pendleton Road

$2 \mathrm{Mill} \mathrm{Cr}$ at Orell Road

3 Pond $\mathrm{Cr}$ at Manslick Road

$5 \mathrm{SF}$ Beargrass $\mathrm{Cr}$ at Winter Avenue

6 SF Beargrass Cr at Trevilian Way

7 MF Beargrass $\mathrm{Cr}$ at Old Cannons Lane

$8 \mathrm{MF}$ Beargrass $\mathrm{Cr}$ at Beals Branch Road

$\begin{array}{lllll}17 & 14 & <1.0 & -- & 0.22^{\star} \\ 17 & 13 & <1.0 & -- & .04 * \\ 16 & 12 & <1.0 & -- & .50 * \\ 16 & 15 & <1.0 & -- & <1.0 \\ 15 & 13 & <1.0 & --- & <1.0 \\ 16 & 14 & <1.0 & -- & <1.0 \\ 12 & 10 & <1.0 & --- & <1.0 \\ 17 & 17 & <.80 & -- & <1.0 \\ 14 & 12 & <1.0 & -- & <1.0 \\ 16 & 13 & <1.0 & -- & .02^{\star} \\ 12 & 12 & <1.0 & --- & <4.0\end{array}$

$0.58^{\star}$
$.31^{\star}$
$1.2^{\star}$
$<4.7$
$<4.7$
$<4.0$
$<4.0$
$<4.0$
$<4.7$
$<5.17^{\star}$
$<5$

$1.5^{\star}$
$2.3^{\star}$
$2.7^{\star}$
$<5.0$
$<5.0$
$<5.0$
$<4.0$
$<5.0$
$<5.0$
$1.3^{\star}$
$<5.0$

---
---
--
--
--
--
--
--
--
--
--

110

114

1.0

9.0

6.0

$<10$

6.0

110

18
15
18
16
16
16
16
16
17
16
16
16
18
18
18
17
16
16
16
17
17
17
14
16
12

$\begin{array}{lll}<6.0 & -- & 11 \\ <6.0 & --- & 7.0 \\ <6.0 & --- & 25 \\ <6.0 & --- & 24 \\ <5.0 & --- & 10^{\star} \\ <6.0 & --- & 35 \\ <6.0 & --- & 26{ }^{\star} \\ <6.0 & --- & 43 \\ <6.0 & --- & 19 \\ <5.0 & --- & 20 \\ <6.0 & --- & 21 \\ <6.0 & --- & 9.2 \\ 15 & --- & 36 \\ <6.0 & --- & 25 \\ <6.0 & --- & 14 * \\ <6.0 & --- & 14 \\ <6.0 & --- & 29 \\ <6.0 & --- & 16 \\ <6.0 & --- & 24 \\ <6.0 & --- & 22 \\ <6.0 & --- & 25 \\ <6.0 & --- & 29 \\ 19 & --- & 43 \\ <6.0 & --- & 13 \\ <6.0 & --- & 17\end{array}$

44
40
60
66
54
50
47
86
52
43
41
52
48
35
51
52
48
44
62
36
36
43
86
28
33

128
55
102
98
165
104
101
140
130
196
81
122
89
66
69
205
117
81
149
55
70
81
187
65
81

$\begin{array}{lr}--- & 1,660 \\ --- & 107 \\ --- & 351 \\ --- & 117 \\ --- & 1,230 \\ --- & 327 \\ --- & 154 \\ --- & 500 \\ --- & 590 \\ --- & 310 \\ --- & 364 \\ --- & 622 \\ --- & 502 \\ --- & 268 \\ --- & 244 \\ --- & 417 \\ --- & 527 \\ --- & 490 \\ --- & 545 \\ --- & 258 \\ --- & 123 \\ --- & 854 \\ --- & 917 \\ --- & 440 \\ --- & 114\end{array}$

Selenium, total, in $\mu \mathrm{g} / \mathrm{L}$ as Se

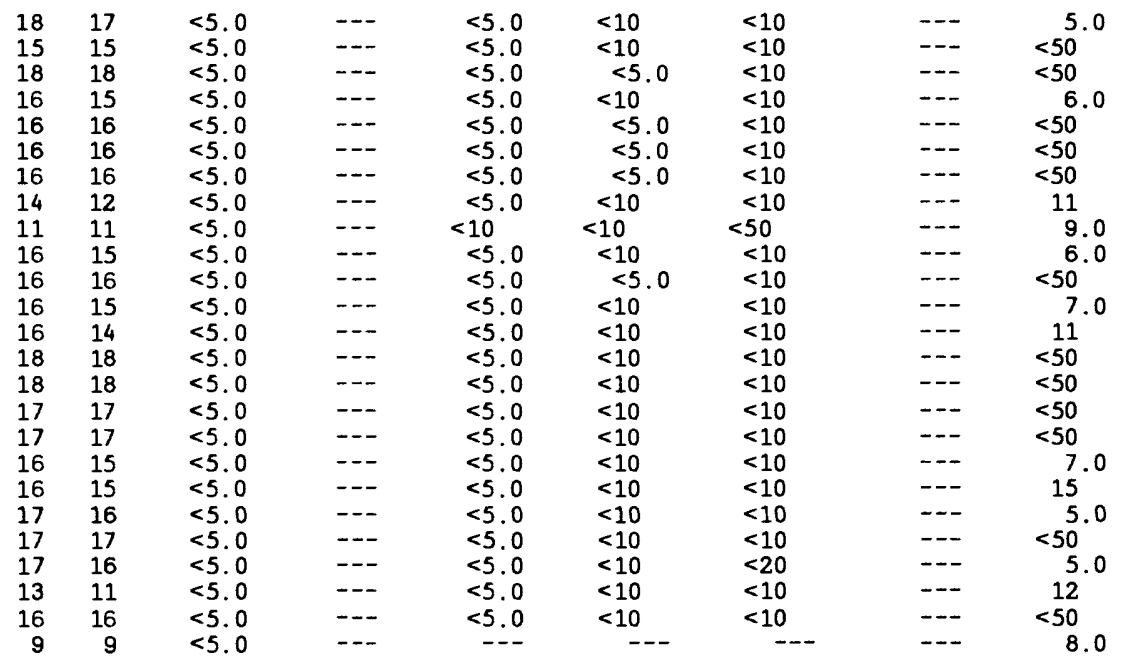

Cyanide, total, in $\mathrm{mg} / \mathrm{L}$ as $\mathrm{Cn}$

$\begin{array}{rrrrr}17 & 16 & <.01 & -- & < \\ 15 & 15 & <.02 & -- & <0 \\ 11 & 8 & <.01 & -- & <0 \\ 16 & 15 & <.01 & -- & <0 \\ 15 & 13 & <.01 & -- & <0 \\ 16 & 15 & <.01 & --- & < \\ 9 & 7 & <.01 & --- & \end{array}$

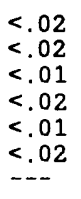

$\begin{array}{ll}<.02 & --- \\ <.02 & --- \\ <.02 & --- \\ <.02 & --- \\ <.01 & --- \\ <.02 & --- \\ --- & ---\end{array}$

.01
$<.02$
$<.02$
.01
.01
.01
$<.02$ 


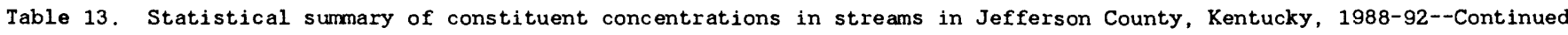
[N, number of observations; $\mathrm{Cr}$, Creek; SF, South Fork; MF, Middle Fork; mg/L, milligrams per liter; ${ }^{\circ} \mathrm{C}$, degrees Celsius; $\mu \mathrm{g} / \mathrm{L}$, micrograms per liter; -- , missing; <, less than; *, value estimated from log-normal fit program; percentiles not computed if fewer than 10 observations; the 10 th and 90 th percentiles are not shown if fewer than 30 observations]

\begin{tabular}{|c|c|c|c|c|c|c|c|}
\hline & & $\begin{array}{c}\text { N of } \\
\text { censored }\end{array}$ & & Value & at indicated percentile & & \\
\hline Site number and name & $\mathbf{N}$ & $\begin{array}{c}\text { data } \\
\text { values }\end{array}$ & Minimum & 25 & $\begin{array}{cc}50 & 75 \\
\text { (median) } & \\
\end{array}$ & 90 & Maximum \\
\hline
\end{tabular}

Cyanide, total, in $\mathrm{mg} / \mathrm{L}$ as Cn--Continued

9 Spring Ditch at Private Drive

10 Muddy Fork at Mockingbird Valley Road

11 Goose $\mathrm{Cr}$ at U.S. Highway 42

12 Little Goose $\mathrm{Cr}$ at U.S. Highway 42

13 Goose $\mathrm{Cr}$ at Old Westport Road

14 Pope Lick at Pope Lick Road

15 Floyds Fork at former State Highway 155

16 Chenoweth Run at Gelhaus Road

17 Fern $\mathrm{Cr}$ at Old Bardstown Road

18 Northern Ditch at Preston Highway

19 Fishpool $\mathrm{Cr}$ at Bost Road

20 Southern Ditch at Minors Lane

21 Floyds Fork at Bardstown Road

22 Cedar $\mathrm{Cr}$ at Thixton Road

23 Pennsylvania Run at Mt. Washington Road

$24 \mathrm{Mill} \mathrm{Cr}$ Cutoff at Dover Road

25 Harrods $\mathrm{Cr}$ at Hunting $\mathrm{Cr}$ Drive

26 Long Run at State Highway 1531

$\begin{array}{ll}12 & 18 \\ 16 & 11 \\ 14 & 10 \\ 10 & \\ 15 & \\ 18 & 10 \\ 18 & 18 \\ 17 & \\ 16 & 11 \\ 17 & 11 \\ 16 & 10 \\ 16 & 11 \\ 16 & 15 \\ 16 & 14 \\ 17 & \\ 11 & \\ 16 & 16 \\ 12 & 11\end{array}$

$\begin{array}{lll}<0.01 & -- & <0.01 \\ <.01 & -- & <.01^{\star} \\ <.01 & -- & <.01 \\ <.01 & -- & <.01 \\ <.01 & -- & .01^{\star} \\ <.01 & --- & <.01^{\star} \\ <.01 & --- & <.02 \\ <.01 & -- & <.01 * \\ <.01 & --- & <.01^{\star} \\ <.01 & --- & <.01^{\star} \\ <.01 & --- & <.01^{\star} \\ <.01 & --- & <.01^{\star} \\ <.01 & --- & <.02 \\ <.01 & --- & <.02 \\ <.01 & -- & <.01^{\star} \\ <.01 & -- & <.01 \\ <.01 & --- & <.02 \\ <.01 & --- & <.02\end{array}$

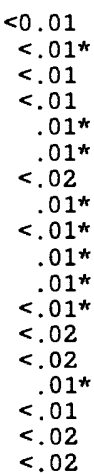

$<0.01$
$.01^{\star}$
.01
.01
$.01^{\star}$
$.01^{\star}$
$<.02$
$.01^{\star}$
$.01^{\star}$
$.01^{\star}$
$.01^{\star}$
$.01^{\star}$
$<.02$
$<.02$
.02
$<.01$
$<.02$
$<.02$

---
---
---
---
---
---
---
---
---
---
---
---
---
---
---
---
---
---

$<0.02$

.03

.01

.03

.05

$<.02$

.04

.02

.02

.01

.07

.02

$<.02$

Chlordane, total, in $\mu \mathrm{g} / \mathrm{L}$

1 Pond $\mathrm{Cr}$ at Pendleton Road

$2 \mathrm{Mill} \mathrm{Cr}$ at Orell Road

3 Pond $\mathrm{Cr}$ at Mans lick Road

5 SF Beargrass $\mathrm{Cr}$ at Winter Avenue

6 SF Beargrass $\mathrm{Cr}$ at Trevilian Way

7 MF Beargrass $\mathrm{Cr}$ at Old Cannons Lane

$8 \mathrm{MF}$ Beargrass $\mathrm{Cr}$ at Beals Branch Road

9 Spring Ditch at Private Drive

10 Muddy Fork at Mockingbird Valley Road

11 Goose $\mathrm{Cr}$ at U.S. Highway 42

12 Little Goose $\mathrm{Cr}$ at U.S. Highway 42

13 Goose $\mathrm{Cr}$ at Old Westport Road

14 Pope Lick at Pope Lick Road

15 Floyds Fork at former State Highway 155

16 Chenoweth Run at Gelhaus Road

17 Fern $\mathrm{Cr}$ at Old Bardstown Road

18 Northern Ditch at Preston Highway

19 Fishpool $\mathrm{Cr}$ at Bost Road

20 Southern Ditch at Minors Lane

21 Floyds Fork at Bardstown Road

22 Cedar $\mathrm{Cr}$ at Thixton Road

23 Pennsylvania Run at Mt. Washington Road

$24 \mathrm{Mill} \mathrm{Cr}$ Cutoff at Dover Road

25 Harrods $\mathrm{Cr}$ at Hunting $\mathrm{Cr}$ Drive

26 Long Run at State Highway 1531

14
12
14
13
13
12
13
14
14
13
13
12
15
15
15
14
13
13
12
14
14
14
12
13
10

$\begin{array}{lll}<3.1 & -- & <3.1 \\ <3.1 & --- & <3.1 \\ <3.1 & --- & <3.1 \\ <3.1 & --- & <3.1 \\ <3.1 & --- & <3.1 \\ <3.1 & --- & <3.1 \\ <3.1 & --- & <3.1 \\ <3.1 & -- & <3.1 \\ <3.1 & --- & <3.1 \\ <3.1 & -- & <3.1 \\ <3.1 & --- & <3.1 \\ <3.1 & --- & <3.1 \\ <3.1 & --- & <3.1 \\ <3.1 & --- & <3.1 \\ <3.1 & --- & <3.1 \\ <3.1 & --- & <3.1 \\ <3.1 & --- & <3.1 \\ <3.1 & --- & <3.1 \\ <3.1 & --- & <3.1 \\ <3.1 & -- & <3.1 \\ <3.1 & --- & <3.1 \\ <3.1 & --- & <3.1 \\ <3.1 & -\cdots & <3.1 \\ <3.1 & --- & <3.1 \\ <3.1 & -\cdots & <3.1 \\ <3.1 & & \end{array}$

$<6.2$

$<6$.

$<6.2$

$<6.2$

$<6.2$

$<6.2$

$<6,2$

$<6.2$

$<6.2$

$<6.2$

$<6.2$

$<6.2$

$<6.2$

$<6.2$

$<6.2$

$<6.2$

$<6.2$

$<6.2$

$<6.2$

$<6.2$

$<6.2$

$<6.2$

$\begin{array}{ll}--- & <6.2 \\ --- & <6.2 \\ --- & <6.2 \\ --- & <6.2 \\ --- & <6.2 \\ --- & <6.2 \\ --- & <6.2 \\ --- & <6.2 \\ --- & <6.2 \\ --- & <6.2 \\ --- & <6.2 \\ --- & <6.2 \\ --- & <6.2 \\ --- & <6.2 \\ --- & <6.3 \\ --- & <6.2 \\ --- & <6.2 \\ --- & <6.2 \\ --- & <6.2 \\ --- & <6.2 \\ --- & <6.2 \\ --- & <6.2 \\ --- & <6.2 \\ --- & <6.2 \\ --- & <6.2\end{array}$

Endrin, total, in $\mu \mathrm{g} / \mathrm{L}$

1 Pond $\mathrm{Cr}$ at Pendleton Road

$2 \mathrm{Mill} \mathrm{Cr}$ at Orell Road

3 Pond $\mathrm{Cr}$ at Mans lick Road

5 SF Beargrass $\mathrm{Cr}$ at Winter Avenue

6 SF Beargrass $\mathrm{Cr}$ at Trevilian Way

7 MF Beargrass $\mathrm{Cr}$ at Old Cannons Lane

$8 \mathrm{MF}$ Beargrass $\mathrm{Cr}$ at Beals Branch Road

9 Spring Ditch at Private Drive

10 Muddy Fork at Mockingbird Valley Road

11 Goose $\mathrm{Cr}_{r}$ at U.S. Highway 42

12 Little Goose $\mathrm{Cr}$ at U.S. Highway 42

13 Goose $\mathrm{Cr}$ at Old Westport Road

14 Pope Lick at Pope Lick Road

15 Floyds Fork at former State Highway 155

16 Chenoweth Run at Gelhaus Road

17 Fern $\mathrm{Cr}$ at Old Bardstown Road

18 Northern Ditch at Preston Highway

19 Fishpool $\mathrm{Cr}$ at Bost Road

20 Southern Ditch at Minors Lane

21 Floyds Fork at Bardstown Road

22 Cedar $\mathrm{Cr}$ at Thixton Road

23 Pennsylvania Run at Mt. Washington Road

$24 \mathrm{Mill} \mathrm{Cr}$ Cutoff at Dover Road

25 Harrods $\mathrm{Cr}$ at Hunting $\mathrm{Cr}$ Drive

26 Long Run at State Highway 1531

\begin{tabular}{|c|c|c|c|c|c|}
\hline 12 & 10 & $<.02$ & --- & $<.04$ & $<.08$ \\
\hline 12 & 12 & $<.02$ & -- & $<.02$ & $<.04$ \\
\hline 14 & 14 & $<.02$ & -- & $<.02$ & $<.05$ \\
\hline 13 & 13 & $<.02$ & --- & $<.02$ & $<.03$ \\
\hline 13 & 12 & $<.02$ & -- & $<.02$ & $<.03$ \\
\hline 12 & 12 & $<.02$ & --- & $<.02$ & .04 \\
\hline 13 & 13 & $<.02$ & --- & $<.02$ & $<.04$ \\
\hline 14 & 13 & $<.02$ & -- & $<.02$ & $<.05$ \\
\hline 13 & 13 & $<.02$ & -- & $<.05$ & $<.05$ \\
\hline 11 & 9 & $<.01$ & --- & $<.02$ & $<.05$ \\
\hline 13 & 12 & $<.02$ & --- & $<.05$ & $<.05$ \\
\hline 12 & 11 & $<.02$ & $-\cdots$ & $<.02$ & $<.05$ \\
\hline 15 & 15 & $<.02$ & --- & $<.02$ & $<.04$ \\
\hline 15 & 14 & $<.02$ & -- & $<.02$ & $<.04$ \\
\hline 15 & 15 & $<.02$ & --- & $<.02$ & $<.04$ \\
\hline 14 & 13 & $<.02$ & --- & $<.02$ & $<.05$ \\
\hline 13 & 12 & $<.01$ & --- & $<.02$ & $<.05$ \\
\hline 13 & 12 & $<.01$ & --- & $<.02$ & $<.04$ \\
\hline 12 & 10 & $<.02$ & --- & $<.04$ & $<.0$ \\
\hline 14 & 13 & $<.02$ & -- & $<.02$ & $<.06$ \\
\hline 14 & 14 & $<.02$ & -- & $<.02$ & $<.06$ \\
\hline 14 & 14 & $<.02$ & -- & $<.02$ & $<.05$ \\
\hline 12 & 11 & $<.02$ & $\cdots$ & $<.02$ & $<.0$ \\
\hline 13 & 13 & $<.02$ & --- & $<.02$ & $<$. \\
\hline 10 & 10 & $<.02$ & --- & $<.02$ & $<$. \\
\hline
\end{tabular}

$<.18$

$\cdots$

$<.39$

$<.18 \quad---<<.39$

$<.16 \quad--1 \quad<.39$

$<.18 \quad--.50$

$<.08 \quad--\quad<.39$

$<.04 \quad---<.39$

.49

$<.06 \quad-\quad<.39$

$\begin{array}{lll}<.18 & -- & .20 \\ <.18 & --- & .18\end{array}$

$<.39 \quad--\quad .04$

$<.16 \quad--<<.39$

$<.18 \quad---\quad .11$

$\begin{array}{lll}<.16 & --- & <.39\end{array}$

$<.08 \quad---\quad .09$

$<.16 \quad--\quad 1.1$

$\begin{array}{rrr}<.39 & -- & .98 \\ <.36 & -- & .30\end{array}$

$<08 \quad--><39$

$<.08 \quad---<.39$

$<.08$
$<.08$
$<.08$

$\begin{array}{lll}<.08 & --- & <.39 \\ <.05 & -- & <.08\end{array}$ 


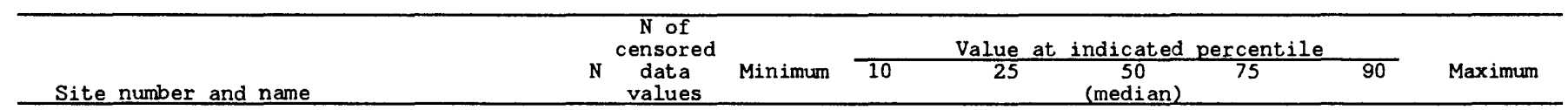

1 Pond $\mathrm{Cr}$ at Pendleton Road

$2 \mathrm{Mill} \mathrm{Cr}$ at Orell Road

3 Pond $\mathrm{Cr}$ at Manslick Road

5 SF Beargrass $\mathrm{Cr}$ at Winter Avenue

$6 \mathrm{SF}$ Beargrass $\mathrm{Cr}$ at Trevilian Way

7 MF Beargrass $\mathrm{Cr}$ at Old Cannons Lane

$8 \mathrm{MF}$ Beargrass $\mathrm{Cr}$ at Beals Branch Road

9 Spring Ditch at Private Drive

10 Muddy Fork at Mockingbird Valley Road

11 Goose $\mathrm{Cr}_{\mathrm{r}}$ at U.S. Highway 42

12 Little Goose $\mathrm{Cr}_{\mathrm{r}}$ at U.S. Highway 42

13 Goose $\mathrm{Cr}$ at Old Westport Road

14 Pope Lick at Pope Lick Road

15 Floyds Fork at former State Highway 155

16 Chenoweth Run at Gelhaus Road

17 Fern $\mathrm{Cr}$ at Old Bardstown Road

18 Northern Ditch at Preston Highway

$19 \mathrm{Fishpool} \mathrm{Cr}$ at Bost Road

20 Southern Ditch at Minors Lane

21 Floyds Fork at Bardstown Road

22 Cedar $\mathrm{Cr}$ at Thixton Road

23 Pennsylvania Run at Mt. Washington Road

24 Mill Cr Cutoff at Dover Road

25 Harrods $\mathrm{Cr}$ at Hunting $\mathrm{Cr}$ Drive

26 Long Run at State Highway 1531

1 Pond $\mathrm{Cr}$ at Pendleton Road

2 Mill $\mathrm{Cr}$ at Orell Road

3 Pond $\mathrm{Cr}$ at Manslick Road

$5 \mathrm{SF}$ Beargrass $\mathrm{Cr}$ at Winter Avenue

6 SF Beargrass $\mathrm{Cr}$ at Trevilian Way

7 MF Beargrass $\mathrm{Cr}$ at Old Cannons Lane

$8 \mathrm{MF}$ Beargrass $\mathrm{Cr}$ at Beals Branch Road

9 Spring Ditch at Private Drive

10 Muddy Fork at Mockingbird Valley Road

11 Goose $\mathrm{Cr}$ at U.S. Highway 42

12 Little Goose $\mathrm{Cr}$ at U.S. Highway 42

13 Goose $\mathrm{Cr}$ at Old Westport Road

14 Pope Lick at Pope Lick Road

15 Floyds Fork at former State Highway 155

16 Chenoweth Run at Gelhaus Road

17 Fern $\mathrm{Cr}_{r}$ at Old Bardstown Road

18 Northern Ditch at Preston Highway

$19 \mathrm{Fishpool} \mathrm{Cr}$ at Bost Road

20 Southern Ditch at Minors Lane

21 Floyds Fork at Bardstown Road

22 Cedar $\mathrm{Cr}$ at Thixton Road

23 Pennsylvania Run at Mt. Washington Road

24 Mill Cr Cutoff at Dover Road

25 Harrods $\mathrm{Cr}$ at Hunting $\mathrm{Cr}$ Drive

26 Long Run at State Highway 1531

1 Pond $\mathrm{Cr}$ at Pendleton Road

2 Mill $\mathrm{Cr}$ at Orell Road

3 Pond $\mathrm{Cr}$ at Manslick Road

$5 \mathrm{SF}$ Beargrass $\mathrm{Cr}$ at Winter Avenue

$6 \mathrm{SF}$ Beargrass $\mathrm{Cr}$ at Trevilian Way

7 MF Beargrass $\mathrm{Cr}$ at Old Cannons Lane

$8 \mathrm{MF}$ Beargrass $\mathrm{Cr}$ at Beals Branch Road

9 Spring Ditch at Private Drive

10 Muddy Fork at Mockingbird Valley Road

11 Goose $\mathrm{Cr}$ at U.S. Highway 42

12 Little Goose $\mathrm{Cr}$ at U.S. Highway 42

13 Goose $\mathrm{Cr}$ at Old Westport Road

14 Pope Lick at Pope Lick Road

15 Floyds Fork at former State Highway 155

16 Chenoweth Run at Gelhaus Road

17 Fern $\mathrm{Cr}$ at Old Bardstown Road

18 Northern Ditch at Preston Highway

19 Fishpool $\mathrm{Cr}$ at Bost Road

20 Southern Ditch at Minors Lane

21 Floyds Fork at Bardstown Road

22 Cedar $\mathrm{Cr}$ at Thixton Road

\section{Lindane, total, in $\mu \mathrm{g} / \mathrm{L}$}

12
12
11
10
13
10
10
12
11
13
13
10
12
12
13
12
11
10
10
12
12
12
11
13
10

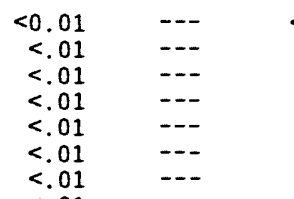

$<0.01$
$<.02$
$<.01^{\star}$

$<0.03$
$<.02$
$.01 *$

$<.02$

$<.02$

$<.01$

$<.01$

$<.01$

$<.01$

$<.01$

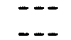

$<.01 *$

$<.01^{*}$

$<.02$

$<.02$

$<.01$

$<.01$

$<.01$

$--$

$<.03$

$<.01$ *

$<.02$

$<.01$

-..

$<.01$

$<.01$

$<.01$

$<.01$

$<.01$

$<.01$

$<.01$

$<.01$

-.-

-.-

$<.02$

$<.01$ *

$<.02$

$<.01 *$

$<.01 *$

$<.01 *$

$<.02$

$<.02$

$<.02$

$<.02$

$<.04$

$<.02$

$<.04$

$.01^{*}$
$.01^{*}$

$<.04$

$<.04$

$<.10$

$<.02^{*}$

.01 *

$<.04$

$.01 *$
$<.02$

$<.04$

$.01 *$

$.01 *$

$<.01^{*}$

$<.04$

$<.04$

$<0.21$

$<.21$

.03

$<.04$

$<.21$

$<.07$
$<.07$

$<.02$ *

$.02 *$

$<.21$

$<.21$

$<.21$ *

$<.02^{*}$

.02 *

$<.21$

$.03 *$

$<.03$
$<.62$

$.03^{*}$

$.05 *$

$.03 *$
$<.08$

$<.08$
$<.21$

$<.09$

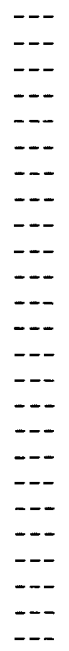

0.39

$<.62$

.08

.05

.12
$<.62$

.22

.58

.02

$<.62$

.09
.13

.21

.61

.13
.04

$<.62$

.22

.36

.49
.30

.04

Methoxychlor, total, in $\mu \mathrm{g} / \mathrm{L}$

$\begin{array}{rr}14 & 13 \\ 12 & 12 \\ 14 & 14 \\ 13 & 13 \\ 11 & \\ 9 & \\ 13 & 12 \\ 14 & 14 \\ 13 & 1 \\ 13 & 12 \\ 13 & 1 \\ 12 & 1 \\ 15 & 15 \\ 15 & 14 \\ 15 & 15 \\ 14 & 13 \\ 13 & 13 \\ 13 & 1 \\ 10 & \\ 14 & 13 \\ 14 & 13 \\ 14 & 1 \\ 12 & 1 \\ 13 & 12 \\ 10 & 10\end{array}$

$<.05$

$<.05$
$<.05$

$<.05$
$<.05$

$<.05$

$<.05$

$<.08$

$<.08$

$<.06$

$<.05$

$<.07$

$<.05$

$<.06$

$<.08$

$<.08$
$<.07$

$<.07$

$<.05$

$<.08$

$<.05$

$<.05$

$<.05$

$<.06$

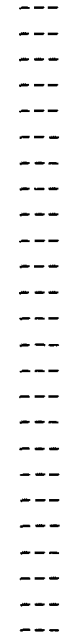

$<.08$

$<.08$

$<.08$

$<08$

$-$

$<.08$

$<.08$

$<.08$

$<.08$

$<.08$

$<.08$

$<.08$

$<.08$

$<.08$

$<.08$

$<.09$

$<.08$

$<.08$

$<.08$

$<.08$

$<.08$
$<.08$

$<.13$

$<.16$

$<.10$

$<.08$

$<.10$

$<.10$

$<.14$

$<.10$

$<.10$

$<.25$

$<.13$

$<.14$

$<.10$

$<.17$
$<.10$

$<10$

$<.10$

$<.11$

$<.17$
$<.17$

$<10$

$<.14$

$<.13$
$<.08$

$<.51$

$<.51$

$<.40$

$<.18$

$<.20$

$<.18$

$<.18$

$<.51$

$<.51$

$<.51$

$<.41$

$<.51$

$<.41$

$<.51$

1.

$<.17$

$<.41$
$<.41$

$<.18$

$<.20$

$<.51$
$<.16$

$---$

$--$

--

$--$

$--$

$--$

$--$

$-$

$--$

$--$

$---$

$--$

-..

$-\cdot$

$--$

$---$

$--$

$--$

Toxaphene, total, in $\mu \mathrm{g} / \mathrm{L}$

$\begin{array}{llllll}14 & 13 & <6.3 & --- & <12 & <12 \\ 12 & 12 & <6.3 & --- & <6.3 & <12 \\ 14 & 13 & <6.3 & --- & <12 & <12 \\ 13 & 13 & <6.3 & --- & <12 & <12 \\ 13 & 13 & <6.3 & --- & <12 & <12 \\ 12 & 11 & <6.3 & --- & <6.3 & <12 \\ 13 & 12 & <6.3 & --- & <12 & <13 \\ 14 & 14 & <6.3 & --- & <12 & <12 \\ 14 & 14 & <6.3 & --- & <6.3 & <12 \\ 13 & 13 & <6.3 & --- & <12 & <12 \\ 13 & 13 & <6.3 & --- & <12 & <12 \\ 12 & 12 & <6.3 & --- & <12 & <12 \\ 15 & 15 & <6.3 & --- & <12 & <12 \\ 15 & 15 & <6.3 & --- & <12 & <12 \\ 15 & 15 & <6.3 & --- & <12 & <12 \\ 14 & 14 & <6.3 & --- & <12 & <12 \\ 13 & 13 & <6.3 & --- & <12 & <12 \\ 13 & 13 & <6.3 & --- & <12 & <12 \\ 12 & 12 & <6.3 & --- & <12 & <12 \\ 14 & 14 & <6.3 & --- & <12 & <12 \\ 14 & 14 & <6.3 & --- & <12 & <12\end{array}$

.12

$<1.3$

$<1.3$ 
Table 14. Estimates of mean annual constituent loads in runoff from urban watersheds of Jefferson County, Kentucky, 1988-92

[Cr, Creek; SF, South Fork; MF, Middle Fork; ${ }^{\circ} \mathrm{C}$, degrees Celsius]

\begin{tabular}{|c|c|c|c|c|c|}
\hline Site number and name & $\begin{array}{c}\text { Number } \\
\text { of } \\
\text { obser- } \\
\text { vations }\end{array}$ & $\begin{array}{l}\text { Mean } \\
\text { annual } \\
\text { load, } \\
\text { in tons }\end{array}$ & $\begin{array}{l}\text { Standard } \\
\text { error } \\
\text { of } \\
\text { regression }\end{array}$ & $\begin{array}{l}\text { Flow duration } \\
\text { of greatest } \\
\text { sampled } \\
\text { discharge, } \\
\text { in percent }\end{array}$ & $\begin{array}{l}\text { Percentage of } \\
\text { load estimated } \\
\text { beyond range } \\
\text { of sampled } \\
\text { discharge }\end{array}$ \\
\hline
\end{tabular}

1 Pond $\mathrm{Cr}$ at Pendleton Road

$2 \mathrm{Mill} \mathrm{Cr}$ at Orell Road

3 Pond $\mathrm{Cr}$ at Manslick Road

5 SF Beargrass $\mathrm{Cr}$ at Winter Avenue

6 SF Beargrass $\mathrm{Cr}$ at Trevilian Way

7 MF Beargrass $\mathrm{Cr}$ at Old Cannons Lane

8 MF Beargrass $\mathrm{Cr}$ at Beals Branch Road

9 Spring Ditch at Private Drive

10 Muddy Fork at Mockingbird Valley Road

11 Goose $\mathrm{Cr}$ at U.S. Highway 42

12 Little Goose $\mathrm{Cr}$ at U.S. Highway 42

13 Goose $\mathrm{Cr}$ at Old Westport Road

14 Pope Lick at Pope Lick Road

15 Floyds Fork at former State Highway 155

16 Chenoweth Run at Gelhaus Road

17 Fern $\mathrm{Cr}$ at Old Bardstown Road

18 Northern Ditch at Preston Highway

19 Fishpool $\mathrm{Cr}$ at Bost Road

20 Southern Ditch at Minors Lane

21 Floyds Fork at Bardstown Road

22 Cedar $\mathrm{Cr}$ at Thixton Road

23 Pennsylvania Run at Mt. Washington Road

$24 \mathrm{Mi} 11 \mathrm{Cr}$ Cutoff at Dover Road

25 Harrods $\mathrm{Cr}$ at Hunting $\mathrm{Cr}$ Drive

26 Long Run at State Highway 1531

\section{Dissolved oxygen}

$\begin{array}{lcc}93 & 880 & 15.7 \\ 87 & 93.0 & 16.7 \\ 95 & 819 & 20.5 \\ 95 & 303 & 27.2 \\ 94 & 201 & 13.5 \\ 95 & 271 & 26.3 \\ 95 & 318 & 26.3 \\ 94 & 35.4 & 30.9 \\ 95 & 82.3 & 15.7 \\ 95 & 135 & 10.6 \\ 96 & 103 & 11.8 \\ 95 & 102 & 20.9 \\ 96 & 39.8 & 16.5 \\ 95 & 2,540 & 19.9 \\ 94 & 253 & 17.7 \\ 95 & 69.5 & 14.2 \\ 96 & 211 & 21.2 \\ 96 & 86.5 & 27.3 \\ 96 & 145 & 26.6 \\ 95 & 4,080 & 17.8 \\ 96 & 171 & 15.5 \\ 96 & 85.7 & 16.0 \\ 84 & 135 & 27.4 \\ 91 & 1,370 & 19.0 \\ 71 & 417 & 14.3\end{array}$

$\begin{array}{rr}0.5 & 9.7 \\ .9 & 16.7 \\ 1.8 & 26.0 \\ .3 & 8.9 \\ .5 & 10.1 \\ 1.3 & 23.0 \\ 1.2 & 27.2 \\ 0 & 0 \\ 1.0 & 18.2 \\ .6 & 6.9 \\ .2 & 4.1 \\ 0 & 0 \\ .3 & 6.8 \\ 0 & 0 \\ 0 & 0 \\ 0 & 0 \\ .1 & 1.7 \\ 0 & 0 \\ 0 & 0 \\ 1.3 & 30.1 \\ .5 & 9.1 \\ .8 & 15.1 \\ .2 & 9.9 \\ 1.2 & 18.8 \\ 0 & 0\end{array}$

\section{Chemical oxygen demand, $0.25 \mathrm{~N}$ dicromate}

3 Pond $C_{r}$ at Manslick Road

5 SF Beargrass $\mathrm{Cr}$ at Winter Avenue

6 SF Beargrass $C r$ at Trevilian Way

7 MF Beargrass $\mathrm{Cr}$ at Old Cannons Lane

8 MF Beargrass $\mathrm{Cr}$ at Beals Branch Road

9 Spring Ditch at Private Drive

10 Muddy Fork at Mockingbird Valley Road

11 Goose $\mathrm{Cr}$ at U.S. Highway 42

12 Little Goose $\mathrm{Cr}$ at U.S. Highway 42

13 Goose $\mathrm{Cr}$ at Old Westport Road

14 Pope Lick at Pope Lick Road

15 Floyds Fork at former State Highway 155

16 Chenoweth Run at Gelhaus Road

17 Fern $\mathrm{Cr}$ at Old Bardstown Road

18 Northern Ditch at Preston Highway

$19 \mathrm{Fishpool} \mathrm{Cr}$ at Bost Road

20 Southern Ditch at Minors Lane

21 Floyds Fork at Bardstown Road

22 Cedar $\mathrm{Cr}$ at Thixton Road

23 Pennsylvania Run at Mt. Washington Road

$24 \mathrm{Mi} 11 \mathrm{Cr}$ Cutoff at Dover Road

25 Harrods $\mathrm{Cr}$ at Hunting $\mathrm{Cr}$ Drive

26 Long Run at State Highway 1531
1 Pond $\mathrm{Cr}$ at Pendleton Road

$2 \mathrm{Mill} \mathrm{Cr}$ at Orell Road

\section{2,000 \\ 55.0}

284

2,630

592

431

324

472

184

172
209

209

248

190

4,060

417

91.7

412
172

172

6,430

343

184
475

475

2,290

783
56.2

54.0

73.2

73.5

66.5

69.8

65.9

67.2

71.9

76.5

62.6

56.1

63.1

60.7

70.4

65.3

65.3
57.4

63.3

63.9

62.6

55.8

49.1

63.8

62.8
13. 3

22.2

36.0

12.3

21.1

25.2

25.2

24.9

9.1

5.0

0
11.

0

0

2. 5

0

29.0

15.1

14.6

12.3

19.1

Biochemical oxygen demand, 5-day at $20^{\circ} \mathrm{C}$

1 Pond $\mathrm{Cr}$ at Pendleton Road

$2 \mathrm{Mill} \mathrm{Cr}$ at Orell Road

3 Pond $\mathrm{Cr}$ at Manslick Road

5 SF Beargrass $\mathrm{Cr}$ at Winter Avenue

$6 \mathrm{SF}$ Beargrass $\mathrm{Cr}$ at Trevilian Way

7 MF Beargrass $\mathrm{Cr}$ at Old Cannons Lane

$8 \mathrm{MF}$ Beargrass $\mathrm{Cr}$ at Beals Branch Road

9 Spring Ditch at Private Drive

10 Muddy Fork at Mockingbird Valley Road

11 Goose $\mathrm{Cr}$ at U.S. Highway 42

12 Little Goose $\mathrm{Cr}$ at U.S. Highway 42

13 Goose $\mathrm{Cr}$ at Old Westport Road

14 Pope Lick at Pope Lick Road

15 Floyds Fork at former State Highway 155

16 Chenoweth Run at Gelhaus Road

17 Fern $\mathrm{Cr}$ at Old Bardstown Road

18 Northern Ditch at Preston Highway

19 Fishpool $\mathrm{Cr}$ at Bost Road

20 Southern Ditch at Minors Lane

21 Floyds Fork at Bardstown Road

22 Cedar $\mathrm{Cr}$ at Thixton Road

23 Pennsylvania Run at Mt. Washington Road

$24 \mathrm{Mill}$ Cr Cutoff at Dover Road

25 Harrods $\mathrm{Cr}$ at Hunting $\mathrm{Cr}$ Drive

26 Long Run at State Highway 1531

$\begin{array}{lcc}89 & 756 & 74.4 \\ 85 & 63.6 & 61.7 \\ 90 & 789 & 65.5 \\ 91 & 317 & 72.2 \\ 91 & 228 & 78.7 \\ 91 & 118 & 62.9 \\ 92 & 118 & 67.3 \\ 91 & 28.6 & 65.4 \\ 90 & 37.2 & 65.1 \\ 90 & 84.0 & 71.3 \\ 91 & 61.9 & 74.5 \\ 90 & 67.9 & 75.6 \\ 92 & 23.6 & 58.5 \\ 91 & 1,000 & 66.1 \\ 90 & 197 & 69.7 \\ 91 & 55.4 & 70.3 \\ 92 & 140 & 72.0 \\ 91 & 42.9 & 66.6 \\ 91 & 81.1 & 51.2 \\ 90 & 1,630 & 60.8 \\ 92 & 114 & 74.0 \\ 92 & 54.5 & 63.2 \\ 79 & 173 & 64.8 \\ 89 & 751 & 68.6 \\ 65 & 235 & 74.3\end{array}$

16.3

18.6

34.0

18.9

25.7

20.7

21.9

19.7

10.7

4. 1

9.6

0

0

2.3

0

24.7

15.2

17.2

15.2

19.9 
Table 14. Estimates of mean annual constituent loads in runoff from urban watersheds of Jefferson County, Kentucky, 1988-92--Continued

[Cr, Creek; SF, South Fork; MF, Middle Fork; ${ }^{\circ} \mathrm{C}$, degrees Celsius]

\begin{tabular}{|c|c|c|c|c|c|}
\hline Site number and name & $\begin{array}{c}\text { Number } \\
\text { of } \\
\text { obser- } \\
\text { vations }\end{array}$ & $\begin{array}{c}\text { Mean } \\
\text { annual } \\
\text { load, } \\
\text { in tons }\end{array}$ & $\begin{array}{l}\text { Standard } \\
\text { error } \\
\text { of } \\
\text { regression }\end{array}$ & $\begin{array}{c}\text { Flow duration } \\
\text { of greatest } \\
\text { sampled } \\
\text { discharge, } \\
\text { in percent }\end{array}$ & $\begin{array}{l}\text { Percentage of } \\
\text { load estimated } \\
\text { beyond range } \\
\text { of sampled } \\
\text { discharge }\end{array}$ \\
\hline
\end{tabular}

1 Pond $\mathrm{Cr}$ at Pendleton Road

$2 \mathrm{Mill} \mathrm{Cr}$ at Orel1 Road

3 Pond $\mathrm{Cr}$ at Manslick Road

5 SF Beargrass $\mathrm{Cr}$ at Winter Avenue

6 SF Beargrass $\mathrm{Cr}$ at Trevilian Way

7 MF Beargrass $\mathrm{Cr}$ at Old Cannons Lane

8 MF Beargrass $\mathrm{Cr}$ at Beals Branch Road

9 Spring Ditch at Private Drive

10 Muddy Fork at Mockingbird Valley Road

11 Goose $\mathrm{Cr}$ at U.S. Highway 42

12 Little Goose $\mathrm{Cr}$ at U.S. Highway 42

13 Goose $\mathrm{Cr}$ at Old Westport Road

14 Pope Lick at Pope Lick Road

15 Floyds Fork at former State Highway 155

16 Chenoweth Run at Gelhaus Road

17 Fern $\mathrm{Cr}$ at Old Bardstown Road

18 Northern Ditch at Preston Highway

19 Fishpool $\mathrm{Cr}$ at Bost Road

20 Southern Ditch at Minors Lane

21 Floyds Fork at Bardstown Road

22 Cedar $\mathrm{Cr}$ at Thixton Road

23 Pennsylvania Run at Mt. Washington Road

$24 \mathrm{Mi} 11 \mathrm{Cr}$ Cutoff at Dover Road

25 Harrods $\mathrm{Cr}$ at Hunting $\mathrm{Cr}$ Drive

26 Long Run at State Highway 1531

Calcium, total as Ca

$\begin{array}{rrrrr}17 & 4,650 & 13.5 & 10.1 & 51.0 \\ 15 & 221 & 39.7 & .9 & 8.9 \\ 18 & 4,630 & 10.6 & 19.4 & 71.7 \\ 16 & 1,430 & 30.0 & 1.2 & 17.7 \\ 16 & 1,070 & 18.4 & 1.5 & 18.2 \\ 16 & 1,360 & 20.2 & 1.3 & 17.8 \\ 16 & 1,640 & 16.2 & 1.6 & 23.3 \\ 16 & 185 & 19.5 & 6.7 & 31.5 \\ 17 & 697 & 20.1 & 3.0 & 27.7 \\ 16 & 721 & 11.7 & 8.0 & 36.0 \\ 16 & 549 & 11.8 & 6.7 & 35.2 \\ 16 & 518 & 15.1 & 10.0 & 39.3 \\ 18 & 296 & 12.3 & 12.9 & 60.8 \\ 18 & 12,300 & 13.8 & 3.0 & 40.6 \\ 18 & 1,540 & 19.2 & 11.9 & 68.8 \\ 17 & 394 & 21.1 & 0 & 0 \\ 16 & 1,020 & 6.8 & 8.3 & 38.3 \\ 16 & 511 & 33.6 & 12.3 & 70.6 \\ 16 & 941 & 16.7 & 18.0 & 78.7 \\ 17 & 22,600 & 24.6 & 7.3 & 64.6 \\ 17 & 1,110 & 36.9 & 8.5 & 54.7 \\ 17 & 402 & 24.5 & 12.4 & 60.9 \\ 15 & 316 & 21.5 & 8.2 & 65.8 \\ 16 & 9,880 & 101.5 & 3.6 & 40.2 \\ 12 & 2,400 & 27.5 & 3.9 & 50.3\end{array}$

\section{Magnesium, total as $\mathrm{Mg}$}

1 Pond $\mathrm{Cr}$ at Pendleton Road

$2 \mathrm{Mill} \mathrm{Cr}$ at Orell Road

3 Pond $\mathrm{Cr}$ at Manslick Road

5 SF Beargrass $\mathrm{Cr}$ at Winter Avenue

6 SF Beargrass $\mathrm{Cr}$ at Trevilian Way

$7 \mathrm{MF}$ Beargrass $\mathrm{Cr}$ at Old Cannons Lane

$8 \mathrm{MF}$ Beargrass $\mathrm{Cr}$ at Beals Branch Road

9 Spring Ditch at Private Drive

10 Muddy Fork at Mockingbird Valley Road

11 Goose $\mathrm{Cr}$ at U.S. Highway 42

12 Little Goose $\mathrm{Cr}$ at U.S. Highway 42

13 Goose $\mathrm{Cr}$ at Old Westport Road

14 Pope Lick at Pope Lick Road

15 Floyds Fork at former State Highway 155

16 Chenoweth Run at Gelhaus Road

17 Ferm $\mathrm{Cr}$ at Old Bardstown Road

18 Northern Ditch at Preston Highway

19 Fishpool $\mathrm{Cr}$ at Bost Road

20 Southern Ditch at Minors Lane

21 Floyds Fork at Bardstown Road

22 Cedar $\mathrm{Cr}$ at Thixton Road

23 Pennsylvania Run at Mt. Washington Road

$24 \mathrm{Mill} \mathrm{Cr}$ Cutoff at Dover Road

25 Harrods $C r$ at Hunting $C r_{r}$ Drive

26 Long Run at State Highway 1531

$\begin{array}{lcr}17 & 1,710 & 15.1 \\ 15 & 68.2 & 48.4 \\ 18 & 1,700 & 8.9 \\ 16 & 408 & 34.7 \\ 16 & 248 & 23.8 \\ 16 & 306 & 19.7 \\ 16 & 350 & 16.8 \\ 16 & 47.3 & 24.4 \\ 17 & 88.1 & 20.9 \\ 16 & 263 & 12.1 \\ 16 & 189 & 11.5 \\ 16 & 203 & 15.1 \\ 18 & 131 & 12.1 \\ 18 & 2,720 & 18.8 \\ 17 & 700 & 16.7 \\ 17 & 168 & 23.1 \\ 16 & 434 & 13.5 \\ 16 & 245 & 47.3 \\ 16 & 430 & 21.6 \\ 17 & 5,450 & 24.5 \\ 17 & 565 & 41.0 \\ 17 & 221 & 19.7 \\ 15 & 101 & 29.6 \\ 16 & 2,650 & 12.9 \\ 12 & 407 & 26.6\end{array}$

$\begin{array}{rr}10.1 & 50.8 \\ 19.9 & 7.6 \\ 1.4 & 71.4 \\ 1.5 & 25.4 \\ 1.3 & 17.7 \\ 1.6 & 18.6 \\ 6.7 & 23.8 \\ 3.0 & 33.4 \\ 8.0 & 23.4 \\ 6.7 & 33.3 \\ 10.0 & 35.6 \\ 12.9 & 37.7 \\ 3.0 & 63.4 \\ 11.9 & 36.6 \\ 0 & 74.8 \\ 8.3 & 0 \\ 12.3 & 42.3 \\ 18.0 & 72.9 \\ 7.3 & 79.3 \\ 8.5 & 58.9 \\ 12.4 & 57.6 \\ 8.2 & 64.4 \\ 3.6 & 65.7 \\ 3.9 & 26.4 \\ & 53.5\end{array}$

Alkalinity, titration to 4.5 , as $\mathrm{CaCO}_{3}$

1 Pond $\mathrm{Cr}$ at Pendleton Road

$2 \mathrm{Mill} \mathrm{Cr}$ at Orell Road

3 Pond $\mathrm{Cr}$ at Manslick Road

5 SF Beargrass $\mathrm{Cr}$ at Winter Avenue

6 SF Beargrass $\mathrm{Cr}$ at Trevilian Way

7 MF Beargrass $\mathrm{Cr}$ at Old Cannons Lane

8 MF Beargrass $\mathrm{Cr}$ at Beals Branch Road

9 Spring Ditch at Private Drive

10 Muddy Fork at Mockingbird Valley Road

11 Goose $\mathrm{Cr}$ at U.S. Highway 42

12 Little Goose $\mathrm{Cr}$ at U.S. Hi ghway 42

13 Goose $\mathrm{Cr}$ at $01 d$ Westport Road

14 Pope Lick at Pope Lick Road

15 Floyds Fork at former State Highway 155

16 Chenoweth Run at Gelhaus Road

17 Fern $\mathrm{Cr}$ at Old Bardstown Road

18 Northern Ditch at Preston Highway

$19 \mathrm{Fishpool} \mathrm{Cr}$ at Bost Road

20 Southern Ditch at Minors Lane

21 Floyds Fork at Bardstown Road

22 Cedar $\mathrm{Cr}$ at Thixton Road

23 Pennsylvania Run at Mt. Washington Road

$24 \mathrm{Mi} 11 \mathrm{Cr}$ Cutoff at Dover Road

25 Harrods $\mathrm{Cr}$ at Hunting $\mathrm{Cr}$ Drive

26 Long Run at State Highway 1531

$\begin{array}{rrr}91 & 12,900 & 26.5 \\ 86 & 1,050 & 49.8 \\ 91 & 12,200 & 17.3 \\ 90 & 4,420 & 27.6 \\ 90 & 3,500 & 30.9 \\ 91 & 4,230 & 22.8 \\ 92 & 4,800 & 22.0 \\ 91 & 495 & 28.1 \\ 90 & 1,820 & 23.9 \\ 91 & 2,110 & 17.6 \\ 91 & 1,570 & 18.8 \\ 90 & 1,570 & 24.4 \\ 92 & 807 & 53.2 \\ 91 & 36,400 & 25.5 \\ 90 & 3,750 & 49.6 \\ 90 & 1,420 & 32.1 \\ 92 & 3,300 & 18.5 \\ 91 & 1,100 & 34.8 \\ 91 & 1,990 & 25.5 \\ 90 & 62,200 & 42.6 \\ 92 & 2,700 & 38.6 \\ 92 & 1,330 & 21.2 \\ 78 & 1,500 & 35.2 \\ 89 & 23,000 & 19.0 \\ 65 & 7,030 & 19.8\end{array}$

1.0
8.9
1.7
7.7
8.2
7.8
33.3
1.5
27.7
6.0
5.2
9.3
60.8
40.6
68.8
0
3.3
70.6
78.7
64.6
54.7
60.9
65.8
0.2
50.3

50.8

7.6

25.4

17.7

23.8

33.4

23. 4

33.3

63.4

36.6
74.8

0

42.3
72.9

79.3

58.9

7. 6

65.7

53.5
8.9

11.0

21.5
8.0

11.2

19.7

20.2

0
17.3

17.3
5.4

5.4
2.7

0

6.8

0

1.6
0

0

26.0

9.5

13.7

10.9

15.0 
Table 14. Estimates of mean annual constituent loads in runoff from urban watersheds of Jefferson County, Kentucky, 1988-92--Continued

[Cr, Creek; SF, South Fork; MF, Middle Fork; ${ }^{\circ} \mathrm{C}$, degrees Celsius ]

\begin{tabular}{|c|c|c|c|c|c|}
\hline Site number and name & $\begin{array}{c}\text { Number } \\
\text { of } \\
\text { obser- } \\
\text { vations }\end{array}$ & $\begin{array}{l}\text { Mean } \\
\text { annual } \\
\text { load, } \\
\text { in tons }\end{array}$ & $\begin{array}{l}\text { Standard } \\
\text { error } \\
\text { of } \\
\text { regression }\end{array}$ & $\begin{array}{c}\text { Flow duration } \\
\text { of greatest } \\
\text { sampled } \\
\text { discharge, } \\
\text { in percent }\end{array}$ & $\begin{array}{l}\text { Percentage of } \\
\text { load estimated } \\
\text { beyond range } \\
\text { of sampled } \\
\text { discharge }\end{array}$ \\
\hline
\end{tabular}

Dissolved solids, residue at $105^{\circ} \mathrm{C}$

1 Pond $\mathrm{Cr}$ at Pendleton Road

$2 \mathrm{Mill} \mathrm{Cr}$ at Orell Road

3 Pond $\mathrm{Cr}$ at Manslick Road

$5 \mathrm{SF}$ Beargrass $\mathrm{Cr}$ at Winter Avenue

6 SF Beargrass $\mathrm{Cr}$ at Trevilian Way

7 MF Beargrass $\mathrm{Cr}$ at Old Cannons Lane

$8 \mathrm{MF}$ Beargrass $\mathrm{Cr}$ at Beals Branch Road

9 Spring Ditch at Private Drive

10 Muddy Fork at Mockingbird Valley Road

11 Goose $\mathrm{Cr}$ at U.S. Highway 42

12 Little Goose $\mathrm{Cr}$ at U.S. Highway 42

13 Goose $\mathrm{Cr}$ at old Westport Road

14 Pope Lick at Pope Lick Road

15 Floyds Fork at former State Highway 155

16 Chenoweth Run at Gelhaus Road

17 Fern $\mathrm{Cr}$ at Old Bardstown Road

18 Northern Ditch at Preston Highway

$19 \mathrm{Fishpool} \mathrm{Cr}$ at Bost Road

20 Southern Ditch at Minors Lane

21 Floyds Fork at Bardstown Road

22 Cedar $\mathrm{Cr}$ at Thixton Road

23 Pennsylvania Run at Mt. Washington Road

$24 \mathrm{Mi} 11 \mathrm{Cr}$ Cutoff at Dover Road

25 Harrods $\mathrm{Cr}$ at Hunting $\mathrm{Cr}$ Drive

26 Long Run at State Highway 1531

$\begin{array}{rrr}91 & 29,800 & 27.7 \\ 86 & 2,000 & 43.0 \\ 91 & 28,000 & 20.1 \\ 91 & 10,500 & 38.5 \\ 91 & 6,920 & 24.3 \\ 91 & 7,220 & 19.7 \\ 91 & 8,690 & 24.5 \\ 91 & 999 & 17.6 \\ 90 & 3,650 & 15.3 \\ 91 & 3,660 & 15.0 \\ 91 & 2,880 & 20.5 \\ 90 & 2,910 & 30.1 \\ 91 & 1,450 & 22.5 \\ 91 & 68,700 & 23.3 \\ 89 & 9,230 & 26.4 \\ 90 & 2,270 & 20.9 \\ 91 & 6,090 & 22.7 \\ 90 & 2,540 & 20.4 \\ 88 & 4,780 & 17.4 \\ 90 & 99,300 & 27.1 \\ 91 & 5,540 & 29.1 \\ 92 & 2,880 & 33.2 \\ 79 & 4,060 & 37.6 \\ 89 & 35,900 & 19.1 \\ 64 & 9,850 & 20.8\end{array}$

0.5

.9
1.8

1.8
.3

.5

1.3

1.2

1.0

.6

$0^{.2}$

$0^{.3}$

0

.1

0

1.3

.5
.8

.2

${ }_{0}^{1.2}$
8.3

12.5

20.6
9.2

10.0

19.7

20.4

0
16.3

5.1

2.8

6.3

0

1.1

0

24.9

7.9
12.7

10.1

13.7

Suspended solids, residue at $105^{\circ} \mathrm{C}$

1 Pond $\mathrm{Cr}$ at Pendleton Road

2 Mill $\mathrm{Cr}$ at Orell Road

3 Pond $\mathrm{Cr}$ at Manslick Road

5 SF Beargrass $\mathrm{Cr}$ at Winter Avenue

$6 \mathrm{SF}$ Beargrass $\mathrm{Cr}$ at Trevilian Way

7 MF Beargrass $\mathrm{Cr}$ at old Cannons Lane

8 MF Beargrass $\mathrm{Cr}$ at Beals Branch Road

9 Spring Ditch at Private Drive

10 Muddy Fork at Mockingbird Valley Road

11 Goose $\mathrm{Cr}$ at U.S. Highway 42

12 Little Goose $\mathrm{Cr}$ at U.S. Highway 42

13 Goose $\mathrm{Cr}$ at Old Westport Road

14 Pope Lick at Pope Lick Road

15 Floyds Fork at former State Highway 155

16 Chenoweth Run at Gelhaus Road

17 Fern $\mathrm{Cr}$ at Old Bardstown Road

18 Northern Ditch at Preston Highway

$19 \mathrm{Fishpool} \mathrm{Cr}$ at Bost Road

20 Southern Ditch at Minors Lane

21 Floyds Fork at Bardstown Road

22 Cedar $\mathrm{Cr}$ at Thixton Road

23 Pennsylvania Run at Mt. Washington Road

24 Mill Cr Cutoff at Dover Road

25 Harrods $\mathrm{Cr}$ at Hunting $\mathrm{Cr}$ Drive

26 Long Run at State Highway 1531

$\begin{array}{lrc}91 & 21,500 & 84.4 \\ 86 & 1,240 & 100 \\ 91 & 22,400 & 73.1 \\ 90 & 7,480 & 86.2 \\ 91 & 4,990 & 98.0 \\ 91 & 839 & 88.6 \\ 91 & 1,190 & 105 \\ 91 & 222 & 90.5 \\ 90 & 580 & 99.2 \\ 91 & 536 & 92.7 \\ 91 & 639 & 98.1 \\ 90 & 587 & 109 \\ 92 & 367 & 101 \\ 91 & 20,100 & 107 \\ 89 & 2,570 & 88.4 \\ 90 & 577 & 100 \\ 92 & 1,470 & 92.3 \\ 91 & 508 & 104 \\ 90 & 1,140 & 97.2 \\ 90 & 64,000 & 90.6 \\ 91 & 455 & 106 \\ 92 & 211 & 102 \\ 79 & 1,440 & 90.8 \\ 89 & 7,870 & 93.7 \\ 65 & 3,770 & 98.3\end{array}$

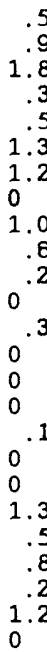

43.4

34.0

56.4

40.0

48.3

43.0

35.7

33.1

19.7

7.2

31.7

.3

1.3

.5

.8
1.2

0

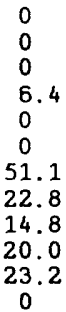

Residue, volatile, nonfilterable

1 Pond $\mathrm{Cr}$ at Pendleton Road

$2 \mathrm{Mill} \mathrm{Cr}$ at Orell Road

3 Pond $\mathrm{Cr}$ at Manslick Road

5 SF Beargrass $\mathrm{Cr}$ at Winter Avenue

$6 \mathrm{SF}$ Beargrass $\mathrm{Cr}$ at Trevilian Way

7 MF Beargrass $\mathrm{Cr}$ at Old Cannons Lane

8 MF Beargrass $\mathrm{Cr}$ at Beals Branch Road

9 Spring Ditch at Private Drive

10 Muddy Fork at Mockingbird Valley Road

11 Goose $\mathrm{Cr}$ at U.S. Highway 42

12 Little Goose $\mathrm{Cr}$ at U.S. Highway 42

13 Goose $\mathrm{Cr}$ at Old Westport Road

14 Pope Lick at Pope Lick Road

15 Floyds Fork at former State Highway 155

16 Chenoweth Run at Gelhaus Road

17 Fern $\mathrm{Cr}$ at Old Bardstown Road

18 Northern Ditch at Preston Highway

19 Fishpool $\mathrm{Cr}$ at Bost Road

20 Southern Ditch at Minors Lane

21 Floyds Fork at Bardstown Road

22 Cedar $\mathrm{Cr}$ at Thixton Road

23 Pennsylvania Run at Mt. Washington Road

24 Mill Cr Cutoff at Dover Road

26 Long Run at State Highway 1531

$\begin{array}{ccc}91 & 3,280 & 106 \\ 86 & 285 & 132 \\ 91 & 4,690 & 116 \\ 90 & 3,240 & 126 \\ 91 & 1,770 & 105 \\ 91 & 534 & 109 \\ 91 & 302 & 103 \\ 91 & 54.2 & 101 \\ 90 & 123 & 106 \\ 91 & 136 & 125 \\ 91 & 167 & 118 \\ 90 & 212 & 130 \\ 92 & 62.9 & 108 \\ 59 & 5,940 & 147 \\ 89 & 743 & 169 \\ 90 & 216 & 115 \\ 92 & 256 & 121 \\ 91 & 147 & 127 \\ 90 & 330 & 132 \\ 90 & 13,400 & 101 \\ 91 & 136 & 120 \\ 92 & 58.2 & 112 \\ 79 & 293 & 104 \\ 65 & 409 & 109\end{array}$

31.0

31.4

52.0

36.4

48.7

38,7

32.3

26.7

6.6

16.5

16.5

0

3.8

0

47.8

14.5

13.3

19.0 
Table 14. Estimates of mean annual constituent loads in runoff from urban watersheds of Jefferson County, Kentucky, 1988-92--Continued

[Cr, Creek; SF, South Fork; MF, Middle Fork; ${ }^{\circ} \mathrm{C}$, degrees Celsius ]

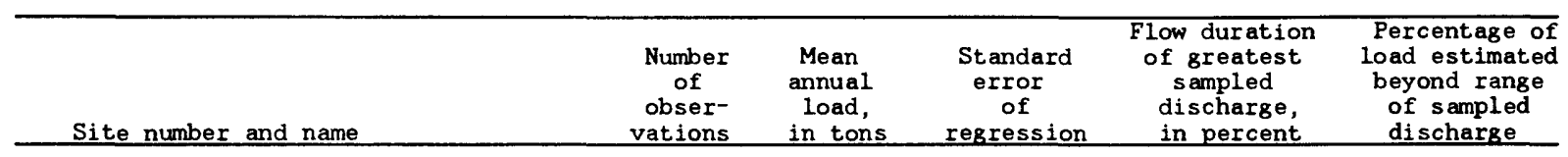

Suspended solids, nonvolatile

1 Pond $\mathrm{Cr}$ at Pendleton Road

2 Mill $\mathrm{Cr}$ at Orell Road

3 Pond $\mathrm{Cr}$ at Manslick Road

$5 \mathrm{SF}$ Beargrass $\mathrm{Cr}$ at Winter Avenue

7 MF Beargrass $\mathrm{Cr}$ at Old Cannons Lane

8 MF Beargrass $\mathrm{Cr}$ at Beals Branch Road

9 Spring Ditch at Private Drive

10 Muddy Fork at Mockingbird Valley Road

11 Goose $\mathrm{Cr}$ at U.S. Highway 42

12 Little Goose $\mathrm{Cr}$ at U.S. Highway 42

13 Goose $\mathrm{Cr}$ at Old Westport Road

14 Pope Lick at Pope Lick Road

15 Floyds Fork at former State Highway 155

16 Chenoweth Run at Gelhaus Road

17 Fern $\mathrm{Cr}$ at Old Bardstown Road

18 Northern Ditch at Preston Highway

$19 \mathrm{Fishpool} \mathrm{Cr}$ at Bost Road

20 Southern Ditch at Minors Lane

21 Floyds Fork at Bardstown Road

23 Pennsylvania Rum at Mt. Washington Road

$24 \mathrm{Mi} 11 \mathrm{Cr}$ Cutoff at Dover Road

26 Long Run at State Highway 1531

$\begin{array}{ccc}20 & 5,210 & 141 \\ 21 & 309 & 120 \\ 21 & 25,400 & 115 \\ 20 & 1,840 & 277 \\ 20 & 553 & 69.5 \\ 21 & 433 & 114 \\ 20 & 40.1 & 65.8 \\ 19 & 8,440 & 94.5 \\ 20 & 88.1 & 100 \\ 20 & 239 & 102 \\ 20 & 466 & 104 \\ 20 & 602 & 235 \\ 20 & 13,300 & 121 \\ 19 & 452 & 245 \\ 20 & 505 & 102 \\ 21 & 176 & 90.0 \\ 19 & 302 & 69.7 \\ 20 & 723 & 108 \\ 19 & 98,700 & 291 \\ 20 & 75.9 & 96.8 \\ 19 & 6,450 & 78.1 \\ 14 & 716 & 92.1\end{array}$

Nitrogen, nitrate, total as $\mathrm{N}$

1 Pond $\mathrm{Cr}$ at Pendleton Road

$2 \mathrm{Mi} 11 \mathrm{Cr}$ at Orell Road

3 Pond $\mathrm{Cr}$ at Manslick Road

5 SF Beargrass $\mathrm{Cr}$ at Winter Avenue

6 SF Beargrass $C_{r}$ at Trevilian Way

7 MF Beargrass $\mathrm{Cr}$ at Old Cannons Lane

8 MF Beargrass $\mathrm{Cr}$ at Beals Branch Road

9 Spring Ditch at Private Drive

10 Muddy Fork at Mockingbird Valley Road

11 Goose $\mathrm{Cr}$ at U.S. Highway 42

12 Little Goose $\mathrm{Cr}$ at U.S. Highway 42

13 Goose $\mathrm{Cr}$ at Old Westport Road

14 Pope Lick at Pope Lick Road

15 Floyds Fork at former State Highway 155

16 Chenoweth Run at Gelhaus Road

17 Fern $\mathrm{Cr}$ at Old Bardstown Road

18 Northern Ditch at Preston Highway

19 Fishpool $\mathrm{Cr}$ at Bost Road

20 Southern Ditch at Minors Lane

21 Floyds Fork at Bardstown Road

22 Cedar $\mathrm{Cr}$ at Thixton Road

23 Pennsylvania Run at Mt. Washington Road

24 Mill Cr Cutoff at Dover Road

25 Harrods $\mathrm{Cr}$ at Hunting $\mathrm{Cr}$ Drive

26 Long Run at State Highway 1531

$\begin{array}{lcr}91 & 212 & 85.8 \\ 85 & 11.7 & 112 \\ 91 & 182 & 85.9 \\ 89 & 56.9 & 88.4 \\ 89 & 40.8 & 86.6 \\ 90 & 48.5 & 99.8 \\ 91 & 54.9 & 93.0 \\ 91 & 8.20 & 89.9 \\ 89 & 22.4 & 92.4 \\ 90 & 40.7 & 45.7 \\ 89 & 38.4 & 46.7 \\ 89 & 29.6 & 63.9 \\ 92 & 10.6 & 83.0 \\ 90 & 310 & 93.1 \\ 89 & 44.5 & 73.1 \\ 90 & 26.0 & 91.4 \\ 92 & 56.9 & 110 \\ 91 & 20.8 & 91.9 \\ 91 & 37.8 & 95.0 \\ 90 & 594 & 111 \\ 92 & 33.6 & 77.6 \\ 92 & 16.8 & 86.4 \\ 77 & 41.8 & 82.6 \\ 88 & 261 & 55.4 \\ 65 & 51.8 & 106\end{array}$

$\begin{array}{rr}.5 & 10.1 \\ .9 & 23.5 \\ 1.8 & 22.2 \\ .3 & 7.6 \\ .5 & 11.6 \\ 1.3 & 21.1 \\ 1.2 & 22.8 \\ 0 & 0 \\ 1.0 & 7.7 \\ .6 & 6.1 \\ .2 & 3.6 \\ 0 & 0 \\ .3 & 3.6 \\ 0 & 0 \\ 0 & 0 \\ 0 & 0 \\ .1 & 0 \\ 0 & 0 \\ 0 & 0 \\ 1.3 & 29.9 \\ .5 & 6.1 \\ .8 & 7.0 \\ .2 & 9.3 \\ 1.2 & 18.8 \\ 0 & 0\end{array}$

1 Pond $C_{r}$ at Pendleton Road

3 Pond $\mathrm{Cr}$ at Manslick Road

$5 \mathrm{SF}$ Beargrass $\mathrm{Cr}$ at Winter Avenue

$6 \mathrm{SF}$ Beargrass $\mathrm{Cr}$ at Trevilian Way

7 MF Beargrass $\mathrm{Cr}$ at Old Cannons Lane

$8 \mathrm{MF}$ Beargrass $\mathrm{Cr}$ at Beals Branch Road

9 Spring Ditch at Private Drive

10 Muddy Fork at Mockingbird Valley Road

11 Goose $\mathrm{Cr}$ at U.S. Highway 42

12 Little Goose $\mathrm{Cr}$ at U.S. Highway 42

13 Goose $\mathrm{Cr}$ at O1d Westport Road

14 Pope Lick at Pope Lick Road

15 Floyds Fork at former State Highway 155

16 Chenoweth Run at Gelhaus Road

17 Fern $\mathrm{Cr}$ at Old Bardstown Road

18 Northern Ditch at Preston Highway

$19 \mathrm{Fishpool} \mathrm{Cr}$ at Bost Road

20 Southern Ditch at Minors Lane

21 Floyds Fork at Bardstown Road

22 Cedar $\mathrm{Cr}$ at Thixton Road

23 Pennsylvania Run at Mt. Washington Road

$24 \mathrm{Mi} 11 \mathrm{Cr}$ Cutoff at Dover Road

25 Harrods $\mathrm{Cr}$ at Hunting $\mathrm{Cr}$ Drive

26 Long Run at State Highway 1531

Nitrogen, nitrite, total as $N$

\begin{tabular}{|c|c|c|c|c|}
\hline $\begin{array}{l}91 \\
91\end{array}$ & $\begin{array}{l}12.1 \\
15.5\end{array}$ & $\begin{array}{l}75.5 \\
66.1\end{array}$ & $\begin{array}{r}.5 \\
1.8\end{array}$ & $\begin{array}{l}19.5 \\
36.3\end{array}$ \\
\hline 90 & 1.76 & 64.5 & .3 & 14.9 \\
\hline 90 & 1.50 & 65.0 & .5 & 27.8 \\
\hline 90 & .54 & 80.0 & 1.3 & 24.6 \\
\hline 91 & 64 & 81.6 & 1.2 & 20.8 \\
\hline 91 & .22 & 75.2 & 0 & 0 \\
\hline 90 & .50 & 76.1 & 1.0 & 19.0 \\
\hline 91 & 1.16 & 91.3 & .6 & 13.5 \\
\hline 91 & .62 & 84.4 & .2 & 7.2 \\
\hline 90 & 1.06 & 104 & 0 & 0 \\
\hline 92 & .38 & 79.6 & .3 & 8.4 \\
\hline 91 & 13.3 & 74.4 & 0 & 0 \\
\hline 90 & 1. 94 & 117 & 0 & 0 \\
\hline 91 & .80 & 101 & 0 & 0 \\
\hline 92 & 1.36 & 61.5 & .1 & 1.4 \\
\hline 91 & 1.12 & 97.8 & 0 & 0 \\
\hline 91 & 1.68 & 72.4 & 0 & 0 \\
\hline 90 & 27.2 & 79.6 & 1.3 & 41.0 \\
\hline 92 & .96 & 81.1 & .5 & 14.8 \\
\hline 92 & 1.18 & 87.3 & .8 & 18.7 \\
\hline 79 & 2.56 & 100 & .2 & 11.8 \\
\hline 89 & 8.34 & 97.4 & 1.2 & 24.5 \\
\hline & & & & \\
\hline
\end{tabular}


Table 14. Estimates of mean annual constituent loads in runoff from urban watersheds of Jefferson County, Kentucky, 1988-92--Continued

[Cr, Creek; SF, South Fork; MF, Middle Fork; ${ }^{\circ} \mathrm{C}$, degrees Celsius ]

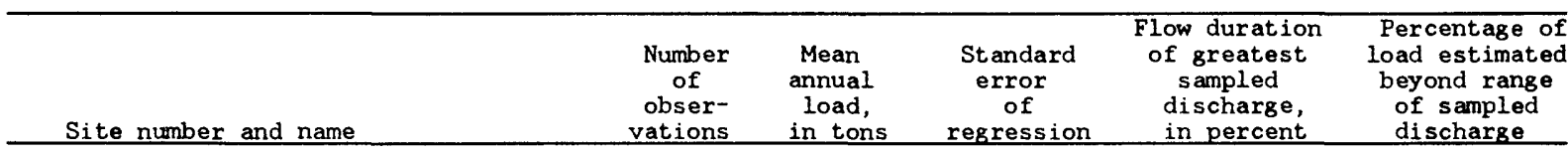

1 Pond $\mathrm{Cr}$ at Pendleton Road

$2 \mathrm{Mill} \mathrm{Cr}$ at Orell Road

3 Pond $\mathrm{Cr}$ at Manslick Road

5 SF Beargrass $\mathrm{Cr}$ at Winter Avenue

6 SF Beargrass $\mathrm{Cr}$ at Trevilian Way

7 MF Beargrass $\mathrm{Cr}$ at Old Cannons Lane

8 MF Beargrass $\mathrm{Cr}$ at Beals Branch Road

9 Spring Ditch at Private Drive

10 Muddy Fork at Mockingbird Valley Road

11 Goose $\mathrm{Cr}$ at U.S. Highway 42

12 Little Goose $\mathrm{Cr}$ at U.S. Highway 42

14 Pope Lick at Pope Lick Road

17 Fern $\mathrm{Cr}$ at old Bardstown Road

18 Northern Ditch at Preston Highway

19 Fishpool $\mathrm{Cr}$ at Bost Road

20 Southern Ditch at Minors Lane

21 Floyds Fork at Bardstown Road

22 Cedar $\mathrm{Cr}$ at Thixton Road

23 Pennsylvania Run at Mt. Washington Road

$24 \mathrm{Mill} \mathrm{Cr}$ Cutoff at Dover Road

Nitrogen, ammonia, total as $\mathrm{N}$

$\begin{array}{lcl}89 & 27.4 & 176 \\ 84 & .68 & 159 \\ 89 & 48.4 & 196 \\ 89 & 13.7 & 187 \\ 89 & 6.20 & 191 \\ 89 & 2.42 & 167 \\ 90 & 2.12 & 170 \\ 88 & .68 & 196 \\ 89 & .78 & 172 \\ 91 & 1.82 & 184 \\ 90 & .72 & 163 \\ 92 & 1.04 & 191 \\ 89 & 2.34 & 186 \\ 89 & 4.52 & 212 \\ 88 & 1.68 & 185 \\ 88 & 4.96 & 186 \\ 90 & 28.1 & 169 \\ 91 & 2.76 & 185 \\ 90 & 3.00 & 190 \\ 78 & 12.1 & 176\end{array}$

0.5
.9
1.8
.3
.5
1.3
1.2
0
1.0
.6
.6
.3
0.1
.1
.2
.4
1.3
.5
.8
2

8.1

16.1

16.4

9.2

19.4

13.4

0

15.8

8.4
8.0

1.8

0.8

6.3

15.3

17.0

11.1

13.6

16.5

\section{Nitrogen, organic, dissolved as $\mathrm{N}$}

1 Pond $\mathrm{Cr}$ at Pendleton Road

$2 \mathrm{Mill} \mathrm{Cr}$ at Orell Road

3 Pond $\mathrm{Cr}$ at Manslick Road

$5 \mathrm{SF}$ Beargrass $\mathrm{Cr}$ at Winter Avenue

$6 \mathrm{SF}$ Beargrass $\mathrm{Cr}$ at Trevilian Way

7 MF Beargrass $\mathrm{Cr}$ at Old Cannons Lane

8 MF Beargrass $\mathrm{Cr}$ at Beals Branch Road

9 Spring Ditch at Private Drive

10 Muddy Fork at Mockingbird Valley Road

11 Goose $\mathrm{Cr}$ at U.S. Highway 42

12 Little Goose $\mathrm{Cr}$ at U.S. Highway 42

13 Goose $\mathrm{Cr}$ at Old Westport Road

14 Pope Lick at Pope Lick Road

15 Floyds Fork at former State Highway 155

16 Chenoweth Run at Gelhaus Road

17 Fern $\mathrm{Cr}$ at Old Bardstown Road

18 Northern Ditch at Preston Highway

$19 \mathrm{Fishpool} \mathrm{Cr}$ at Bost Road

20 Southern Ditch at Minors Lane

21 Floyds Fork at Bardstown Road

22 Cedar $\mathrm{Cr}$ at Thixton Road

23 Pennsylvania Run at Mt. Washington Road

24 Mill Cr Cutoff at Dover Road

25 Harrods $\mathrm{Cr}$ at Hunting $\mathrm{Cr}$ Drive

26 Long Run at State Highway 1531

1 Pond $\mathrm{Cr}$ at Pendleton Road

$2 \mathrm{Mill} \mathrm{Cr}$ at Orell Road

3 Pond $\mathrm{Cr}$ at Manslick Road

$5 \mathrm{SF}$ Beargrass $\mathrm{Cr}$ at Winter Avenue

$6 \mathrm{SF}$ Beargrass $\mathrm{Cr}$ at Trevilian Way

7 MF Beargrass $\mathrm{Cr}$ at Old Cannons Lane

$8 \mathrm{MF}$ Beargrass $\mathrm{Cr}$ at Beals Branch Road

9 Spring Ditch at Private Drive

10 Muddy Fork at Mockingbird Valley Koad

11 Goose $\mathrm{Cr}$ at U.S. Highway 42

12 Little Goose $\mathrm{Cr}$ at U.S. Highway 42

13 Goose $\mathrm{Cr}$ at Old Westport Road

14 Pope Lick at Pope Lick Road

15 Floyds Fork at former State Highway 155

16 Chenoweth Run at Gelhaus Road

17 Fern $\mathrm{Cr}$ at 01d Bardstown Road

18 Northern Ditch at Preston Highway

19 Fishpool $\mathrm{Cr}$ at Bost Road

20 Southern Ditch at Minors Lane

21 Floyds Fork at Bardstown Road

22 Cedar $\mathrm{Cr}$ at Thixton Road

23 Pennsylvania Run at Mt. Washington Road

24 Mill Cr Cutoff at Dover Road

25 Harrods $\mathrm{Cr}$ at Hunting $\mathrm{Cr}$ Drive

26 Long Run at State Highway 1531

$\begin{array}{lcl}90 & 57.0 & 132 \\ 86 & 4.84 & 136 \\ 90 & 54.0 & 128 \\ 91 & 30.3 & 135 \\ 91 & 14.3 & 132 \\ 91 & 8.58 & 141 \\ 92 & 9.14 & 142 \\ 90 & 4.24 & 139 \\ 90 & 2.72 & 137 \\ 90 & 6.30 & 142 \\ 89 & 3.62 & 145 \\ 90 & 7.40 & 155 \\ 92 & 3.64 & 119 \\ 90 & 188 & 113 \\ 90 & 13.1 & 151 \\ 91 & 4.18 & 129 \\ 91 & 18.8 & 148 \\ 89 & 5.60 & 134 \\ 90 & 12.1 & 140 \\ 89 & 198 & 129 \\ 92 & 11.0 & 117 \\ 91 & 9.00 & 142 \\ 79 & 26.2 & 133 \\ 89 & 89.7 & 124 \\ 65 & 33.2 & 111 \\ & & \end{array}$

12.2

16.1

2.2. 8

16.2

16.8

21.1

19.8

0
14.6

9.1

2.6

10.8

0.3

0

0

0

3.0

5.4

13.8

28.3

13.3
18.7

13.9

25.2

$\frac{\text { Phosphate, total as PO }}{91} \quad 135 \quad 57.4$

$\begin{array}{lll}84 & 11.9 & 76.4\end{array}$

$91 \quad 149.9 \quad 54.7$

$\begin{array}{lll}90 & 24.1 & 84.7\end{array}$

$\begin{array}{lll}89 & 19.2 & 89.6 \\ 85 & 13.6 & 93.2\end{array}$

$\begin{array}{lll}85 & 13.6 & 93.2\end{array}$

$\begin{array}{lll}87 & 8.80 & 75.0\end{array}$

$\begin{array}{lll}88 & 5.70 & 69.7 \\ 89 & 9.44 & 54.8\end{array}$

$90 \quad 23.3 \quad 53.4$

$91 \quad 17.0 \quad 59.4$

$\begin{array}{lll}89 & 19.9 & 70.2\end{array}$

$91 \quad 7.36 \quad 67.3$

$\begin{array}{lll}90 & 168 & 76.1\end{array}$

$50.4 \quad 61.1$

$15.8 \quad 71.9$

$44.3 \quad 64.2$

$\begin{array}{ll}13.6 & 68.4 \\ 22.0 & 70.4\end{array}$

247

24.7

$91 \quad 14.0 \quad 68.9$

$\begin{array}{lll}78 & 51.8 & 99.4\end{array}$

97.8

68.5
63.5

$\begin{array}{lll}88 & 97.8 & 83.7\end{array}$


Table 14. Estimates of mean annual constituent loads in runoff from urban watersheds of Jefferson County, Kentucky, 1988-92--Continued

[Cr, Creek; SF, South Fork; MF, Middle Fork; ${ }^{\circ} \mathrm{C}$, degrees Celsius]

\begin{tabular}{|c|c|c|c|c|c|}
\hline Site number and name & $\begin{array}{l}\text { Number } \\
\text { of } \\
\text { obser- } \\
\text { vations }\end{array}$ & $\begin{array}{l}\text { Mean } \\
\text { annual } \\
\text { load, } \\
\text { in tons }\end{array}$ & $\begin{array}{l}\text { Standard } \\
\text { error } \\
\text { of } \\
\text { regression }\end{array}$ & $\begin{array}{c}\text { Flow duration } \\
\text { of greatest } \\
\text { sampled } \\
\text { discharge, } \\
\text { in percent }\end{array}$ & $\begin{array}{l}\text { Percentage of } \\
\text { load est imated } \\
\text { beyond range } \\
\text { of sampled } \\
\text { discharge }\end{array}$ \\
\hline
\end{tabular}

1 Pond $\mathrm{Cr}$ at Pendleton Road

$2 \mathrm{Mill} \mathrm{Cr}$ at Orell Road

3 Pond $\mathrm{Cr}$ at Manslick Road

5 SF Beargrass $\mathrm{Cr}$ at Winter Avenue

$6 \mathrm{SF}$ Beargrass $\mathrm{Cr}$ at Trevilian Way

7 MF Beargrass $\mathrm{Cr}$ at Old Cannons Lane

8 MF Beargrass $\mathrm{Cr}$ at Beals Branch Road

9 Spring Ditch at Private Drive

10 Muddy Fork at Mockingbird Valley Road

11 Goose $\mathrm{Cr}$ at U.S. Highway 42

12 Little Goose $\mathrm{Cr}$ at U.S. Hi ghway 42

13 Goose $\mathrm{Cr}$ at Old Westport Road

14 Pope Lick at Pope Lick Road

15 Floyds Fork at former State Highway 155

16 Chenoweth Run at Gelhaus Road

17 Fern $\mathrm{Cr}$ at Old Bardstown Road

18 Northern Ditch at Preston Highway

$19 \mathrm{Fishpool} \mathrm{Cr}$ at Bost Road

20 Southern Ditch at Minors Lane

21 Floyds Fork at Bardstown Road

22 Cedar $\mathrm{Cr}$ at Thixton Road

23 Pennsylvania Run at Mt. Washington Road

$24 \mathrm{Mill} \mathrm{Cr}$ Cutoff at Dover Road

25 Harrods $\mathrm{Cr}$ at Hunting $\mathrm{Cr}$ Drive

26 Long Run at State Highway 1531

\section{Phosphorus, total as P}

$\begin{array}{lcl}88 & 57.8 & 65.4 \\ 83 & 6.64 & 85.8 \\ 86 & 60.4 & 60.3 \\ 89 & 17.3 & 81.8 \\ 87 & 8.44 & 78.7 \\ 88 & 7.04 & 82.4 \\ 89 & 6.14 & 85.1 \\ 86 & 2.62 & 60.5 \\ 88 & 4.82 & 63.3 \\ 88 & 10.7 & 63.7 \\ 88 & 7.82 & 73.1 \\ 89 & 9.08 & 70.1 \\ 88 & 4.22 & 73.3 \\ 86 & 98.4 & 63.7 \\ 86 & 16.8 & 73.2 \\ 89 & 8.42 & 72.3 \\ 89 & 21.0 & 69.0 \\ 87 & 6.42 & 68.1 \\ 89 & 10.6 & 66.1 \\ 86 & 135 & 76.0 \\ 89 & 10.5 & 63.5 \\ 90 & 6.48 & 72.5 \\ 78 & 16.5 & 91.9 \\ 87 & 49.2 & 86.9 \\ 65 & 24.7 & 94.4\end{array}$

$\begin{array}{rr}0.5 & 8.2 \\ .9 & 29.4 \\ 1.8 & 19.2 \\ .3 & 24.2 \\ .5 & 35.8 \\ 1.3 & 37.4 \\ 1.2 & 26.2 \\ 0 & 0 \\ 1.0 & 6.0 \\ .6 & 4.0 \\ .6 & 4.7 \\ 0 & 0 \\ .3 & 5.4 \\ 0 & 0 \\ 8.6 & 29.4 \\ 0 & 0 \\ .1 & 0.5 \\ 0 & 0 \\ 0 & 0 \\ 1.3 & 21.6 \\ .5 & 4.1 \\ .8 & 5.8 \\ .2 & 9.5 \\ 1.2 & 18.0 \\ 0 & 0\end{array}$

Phosphorus, orthophosphate, total as P

1 Pond $\mathrm{Cr}$ at Pendleton Road

$2 \mathrm{Mill} \mathrm{Cr}$ at Orell Road

3 Pond $\mathrm{Cr}$ at Manslick Road

5 SF Beargrass $\mathrm{Cr}$ at Winter Avenue

6 SF Beargrass $\mathrm{Cr}$ at Trevilian Way

7 MF Beargrass $\mathrm{Cr}$ at Old Cannons Lane

8 MF Beargrass $\mathrm{Cr}$ at Beals Branch Road

9 Spring Ditch at Private Drive

10 Muddy Fork at Mockingbird Valley Road

11 Goose $\mathrm{Cr}$ at U.S. Highway 42

12 Little Goose $\mathrm{Cr}$ at U.S. Hi ghway 42

13 Goose $\mathrm{Cr}$ at Old Westport Road

14 Pope Lick at Pope Lick Road

15 Floyds Fork at former State Highway 155

16 Chenoweth Run at Gelhaus Road

17 Fern $\mathrm{Cr}$ at Old Bardstown Road

18 Northern Ditch at Preston Highway

19 Fishpool $\mathrm{Cr}$ at Bost Road

20 Southern Ditch at Minors Lane

21 Floyds Fork at Bardstown Road

22 Cedar $\mathrm{Cr}$ at Thixton Road

23 Pennsylvania Run at Mt. Washington Road

$24 \mathrm{Mill} \mathrm{Cr}$ Cutoff at Dover Road

25 Harrods $\mathrm{Cr}$ at Hunting $\mathrm{Cr}$ Drive

26 Long Run at State Highway 1531

1 Pond $\mathrm{Cr}$ at Pendleton Road

$2 \mathrm{Mill} \mathrm{Cr}$ at Orell Road

3 Pond $\mathrm{Cr}$ at Manslick Road

5 SF Beargrass $\mathrm{Cr}$ at Winter Avenue

$6 \mathrm{SF}$ Beargrass $\mathrm{Cr}$ at Trevilian Way

$7 \mathrm{MF}$ Beargrass $\mathrm{Cr}$ at Old Cannons Lane

$8 \mathrm{MF}$ Beargrass $\mathrm{Cr}$ at Beals Branch Road

9 Spring Ditch at Private Drive

10 Muddy Fork at Mockingbird Valley Road

11 Goose $\mathrm{Cr}$ at U.S. Highway 42

12 Little Goose $\mathrm{Cr}$ at U.S. Highway 42

13 Goose $\mathrm{Cr}$ at Old Westport Road

14 Pope Lick at Pope Lick Road

15 Floyds Fork at former State Highway 155

16 Chenoweth Run at Gelhaus Road

17 Fern $\mathrm{Cr}$ at Old Bardstown Road

18 Northern Ditch at Preston Highway

19 Fishpool $\mathrm{Cr}$ at Bost Road

20 Southern Ditch at Minors Lane

21 Floyds Fork at Bardstown Road

22 Cedar $\mathrm{Cr}$ at Thixton Road

23 Pennsylvania Run at Mt. Washington Road

$24 \mathrm{Mill} \mathrm{Cr}$ Cutoff at Dover Road

25 Harrods $\mathrm{Cr}$ at Hunting $\mathrm{Cr}$ Drive

26 Long Run at State Highway 1531

$\begin{array}{lcr}91 & 43.9 & 57.4 \\ 85 & 4.14 & 81.8 \\ 91 & 48.6 & 54.7 \\ 90 & 7.86 & 84.7 \\ 90 & 6.42 & 92.1 \\ 89 & 4.20 & 104 \\ 90 & 2.74 & 85.7 \\ 90 & 1.82 & 93.1 \\ 89 & 3.08 & 54.8 \\ 90 & 7.60 & 53.4 \\ 91 & 5.54 & 59.4 \\ 89 & 6.48 & 70.2 \\ 91 & 2.40 & 67.3 \\ 90 & 54.8 & 76.2 \\ 87 & 16.4 & 61.1 \\ 90 & 5.14 & 71.9 \\ 90 & 14.0 & 82.0 \\ 90 & 4.42 & 83.8 \\ 91 & 7.16 & 84.0 \\ 89 & 80.5 & 68.5 \\ 91 & 8.04 & 63.5 \\ 91 & 4.56 & 69.0 \\ 78 & 16.9 & 99.3 \\ 88 & 31.9 & 83.7 \\ 65 & 22.3 & 100\end{array}$

1
1
1
0
1
0
0
0

0

1. 3

.5

1.2

0

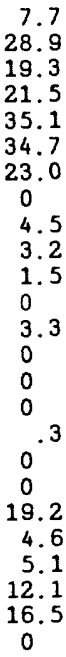

Barium, total as Ba

18

15

16

16

16

16

17

16

16

18

18

18

17

16

16
16

17

17

17

15

12

$\begin{array}{rr}4.52 & 31.8 \\ .40 & 35.9 \\ 4.32 & 17.8 \\ 1.98 & 23.1 \\ 1.22 & 15.4 \\ 1.12 & 13.7 \\ 1.46 & 16.5 \\ .14 & 15.4 \\ .46 & 20.2 \\ .52 & 24.9 \\ .54 & 30.5 \\ .40 & 30.9 \\ .14 & 89.1 \\ 8.16 & 36.0 \\ 1.06 & 44.9 \\ .60 & 34.5 \\ .98 & 17.7 \\ .46 & 46.4 \\ .70 & 15.9 \\ 17.9 & 14.7 \\ .78 & 23.5 \\ .32 & 17.6 \\ .62 & 15.7 \\ 6.18 & 62.1 \\ .90 & 42.0\end{array}$

10.1

19.

19.4
1.2
1.5

1. 3

1. 6

6.7

3.0

8.0

6.7

10.0

12.9

3.0

11.9

8.3
12.3
18.0

18.0

7.3

8.5

12.4

8.2

3. 6

3.9

60.6

16.0

74.9

27.4

24.2

22.2

28.6

37.0

33. 3

34.0

41.6

34.1

50.8

44.0

68.6

0

49. 4

75.0

77.7

68.7

58.6

58.5

83.9

25.8

43.8 
Table 14. Estimates of mean annual constituent loads in runoff from urban watersheds of Jefferson County, Kentucky, 1988-92--Continued

[Cr, Creek; SF, South Fork; MF, Middle Fork; ${ }^{\circ} \mathrm{C}$, degrees Celsius]

\begin{tabular}{|c|c|c|c|c|c|}
\hline Site number and name & $\begin{array}{c}\text { Number } \\
\text { of } \\
\text { obser- } \\
\text { vations }\end{array}$ & $\begin{array}{l}\text { Mean } \\
\text { annual } \\
\text { load, } \\
\text { in tons }\end{array}$ & $\begin{array}{l}\text { Standard } \\
\text { error } \\
\text { of } \\
\text { regression }\end{array}$ & $\begin{array}{c}\text { Flow duration } \\
\text { of greatest } \\
\text { sampled } \\
\text { discharge, } \\
\text { in percent }\end{array}$ & $\begin{array}{l}\text { Percentage of } \\
\text { load estimated } \\
\text { beyond range } \\
\text { of sampled } \\
\text { discharge }\end{array}$ \\
\hline
\end{tabular}

1 Pond $\mathrm{Cr}$ at Pendleton Road

3 Pond $\mathrm{Cr}$ at Manslick Road

9 Spring Ditch at Private Drive

Chromium, total as $\mathrm{Cr}$

1 Pond $\mathrm{Cr}$ at Pendleton Road

3 Pond $\mathrm{Cr}$ at Manslick Road

$5 \mathrm{SF}$ Beargrass $\mathrm{Cr}$ at Winter Avenue

$8 \mathrm{MF}$ Beargrass $\mathrm{Cr}$ at Beals Branch Road

9 Spring Ditch at Private Drive

11 Goose $\mathrm{Cr}$ at U.S. Highway 42

13 Goose $\mathrm{Cr}$ at Old Westport Road

14 Pope Lick at Pope Lick Road

15 Floyds Fork at former State Highway 155

16 Chenoweth Run at Gelhaus Road

17 Fern $\mathrm{Cr}$ at Old Bardstown Road

18 Northern Ditch at Preston Highway

20 Southern Ditch at Minors Lane

21 Floyds Fork at Bardstown Road

22 Cedar $\mathrm{Cr}$ at Thixton Road

23 Pennsylvania Run at Mt. Washington Road

$24 \mathrm{Mi} 11 \mathrm{Cr}$ Cutoff at Dover Road

26 Long Run at State Highway 1531

$2 \mathrm{Mill} \mathrm{Cr}$ at Orell Road

3 Pond $\mathrm{Cr}$ at Manslick Road

$5 \mathrm{SF}$ Beargrass $\mathrm{Cr}$ at Winter Avenue

$6 \mathrm{SF}$ Beargrass $\mathrm{Cr}$ at Trevilian Way

7 MF Beargrass $\mathrm{Cr}$ at Old Cannons Lane

$8 \mathrm{MF}$ Beargrass $\mathrm{Cr}$ at Beals Branch Road

9 Spring Ditch at Private Drive

10 Muddy Fork at Mockingbird Valley Road

11 Goose $\mathrm{Cr}$ at U.S. Highway 42

12 Little Goose $\mathrm{Cr}$ at U.S. Highway 42

13 Goose $\mathrm{Cr}$ at Old Westport Road

15 Floyds Fork at former State Highway 155

16 Chenoweth Run at Gelhaus Road

17 Fern $\mathrm{Cr}$ at Old Bardstown Road

18 Northern Ditch at Preston Highway

19 Fishpool $\mathrm{Cr}$ at Bost Road

20 Southern Ditch at Minors Lane

21 Floyds Fork at Bardstown Road

22 Cedar $\mathrm{Cr}$ at Thixton Road

23 Pennsylvania Run at Mt. Washington Road

$24 \mathrm{Mill} \mathrm{Cr}$ Cutoff at Dover Road

25 Harrods $\mathrm{Cr}$ at Hunting $\mathrm{Cr}$ Drive

26 Long Run at State Highway 1531

21 Floyds Fork at Bardstown Road

22 Cedar $\mathrm{Cr}$ at Thixton Road

23 Pennsylvania Run at Mt. Washington Road

$\begin{array}{rrr}18 & 2.74 & 118 \\ 18 & 1.24 & 110 \\ 16 & 12 & 78\end{array}$

.1278 .9

10.1

19.4
6.7

62.3

63.8

Copper, total recoverable as $\mathrm{Cu}$

$155 \quad 18$

$\begin{array}{rrrrr}18 & 1.90 & 87.1 & 10.1 & 64.1 \\ 18 & 2.22 & 115 & 19.4 & 81.4 \\ 16 & .70 & 93.6 & 1.2 & 35.3 \\ 16 & 1.24 & 94.6 & 1.6 & 43.4 \\ 16 & .06 & 84.1 & 6.7 & 36.1 \\ 16 & .22 & 107 & 8.0 & 34.4 \\ 16 & .12 & 98.1 & 10.0 & 26.1 \\ 18 & .04 & 84.4 & 12.9 & 44.2 \\ 18 & 5.80 & 87.7 & 3.0 & 51.1 \\ 18 & .40 & 112 & 11.9 & 64.5 \\ 17 & .14 & 115 & 0 & 0 \\ 16 & .20 & 67.3 & 8.3 & 21.9 \\ 16 & .22 & 114 & 18.0 & 66.3 \\ 17 & 3.16 & 80.0 & 7.3 & 57.9 \\ 17 & .18 & 92.2 & 8.5 & 48.8 \\ 17 & .12 & 104 & 12.4 & 55.1 \\ 15 & .08 & 101 & 8.2 & 64.6 \\ 12 & 1.90 & 72.6 & 3.9 & 68.6\end{array}$

\section{Iron, total as $\mathrm{Fe}$}

$\begin{array}{lcc}15 & 83.2 & 111 \\ 18 & 251 & 63.8 \\ 16 & 857 & 79.5 \\ 16 & 36.7 & 205 \\ 16 & 31.4 & 139 \\ 16 & 55.9 & 155 \\ 16 & 10.3 & 140 \\ 17 & 282 & 105 \\ 16 & 7.92 & 168 \\ 16 & 25.5 & 101 \\ 16 & 7.48 & 173 \\ 18 & 3,000 & 140 \\ 18 & 5.88 & 150 \\ 17 & 73.1 & 164 \\ 16 & 39.2 & 67.2 \\ 16 & 4.30 & 117 \\ 16 & 8.18 & 51.9 \\ 17 & 1,610 & 160 \\ 17 & 6.30 & 135 \\ 17 & 3.72 & 166 \\ 15 & 128 & 66.4 \\ 16 & 1.860 & 153 \\ 12 & 45.7 & 109\end{array}$

.9
19.4
1.2
1.5
1.3
1.6
6.7
3.0
8.0
6.7
10.0
3.0
11.9
0
8.3
12.3
18.0
7.3
8.5
12.4
8.2
3.6
3.9

33. 2

85.4

74.8

34.4

45.5

46.9

60.8

85.2

48.5

60.0

38.6

62.4

35.3

0

82.5

73.1

70.4

84.6

51.2

34.9

93.9

71.0

56.2

Mercury, total recoverable as $\mathrm{Hg}$

6 SF Beargrass $\mathrm{Cr}$ at Trevilian Way

9 Spring Ditch at Private Drive

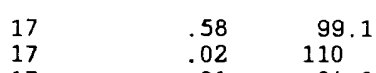

$\begin{array}{rrr}17 & .02 & 110 \\ 17 & <.01 & 84.9\end{array}$

Nickel, total as $\mathrm{Ni}$

$\begin{array}{rr}7.3 & 84.5 \\ 8.5 & 60.9 \\ 12.4 & 46.3\end{array}$

1.5
6.7

25.7

46.5 
Table 14. Estimates of mean annual constituent loads in runoff from urban watersheds of Jefferson County, Kentucky, 1988-92--Continued

[Cr, Creek; SF, South Fork; MF, Middle Fork; ${ }^{\circ} \mathrm{C}$, degrees Celsius]

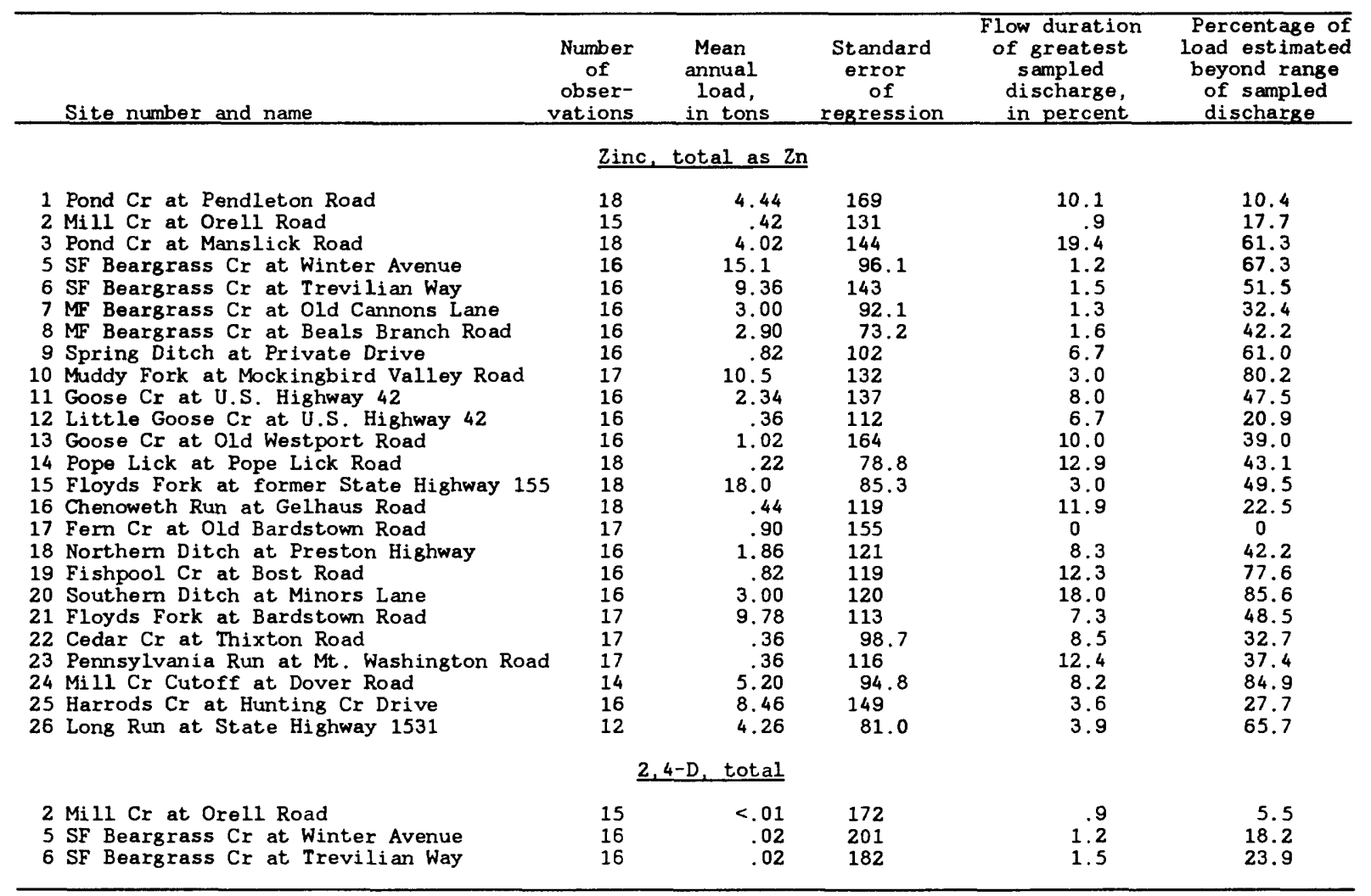


Table 15. Estimates of mean annual loads of constituents in base flow in urban watersheds of Jefferson County, Kentucky, 1988-92

[Cr, Creek; SF, South Fork; MF, Middle Fork; ${ }^{\circ} \mathrm{C}$, degrees Celsius]

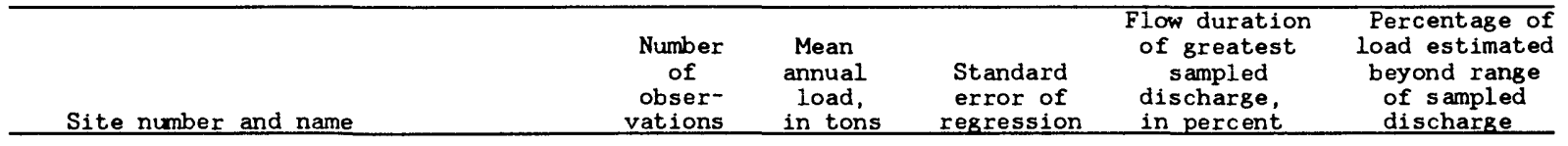

1 Pond $\mathrm{Cr}$ at Pendleton Road

$2 \mathrm{Mill} \mathrm{Cr}$ at Orell Road

3 Pond $\mathrm{Cr}$ at Manslick Road

5 SF Beargrass $\mathrm{Cr}$ at Winter Avenue

$6 \mathrm{SF}$ Beargrass $\mathrm{Cr}$ at Trevilian Way

7 MF Beargrass $\mathrm{Cr}$ at O1d Cannons Lane

$8 \mathrm{MF}$ Beargrass $\mathrm{Cr}$ at Beals Branch Road

9 Spring Ditch at Private Drive

10 Muddy Fork at Mockingbird Valley Road

11 Goose $\mathrm{Cr}$ at U.S. Highway 42

12 Little Goose $\mathrm{Cr}$ at U.S. Highway 42

13 Goose $\mathrm{Cr}$ at old Westport Road

14 Pope Lick at Pope Lick Road

15 Floyds Fork at former State Highway 155

16 Chenoweth Run at Gelhaus Road

17 Fern $\mathrm{Cr}$ at Old Bardstown Road

18 Northern Ditch at Preston Highway

$19 \mathrm{Fishpool} \mathrm{Cr}$ at Bost Road

20 Southern Ditch at Minors Lane

21 Floyds Fork at Bardstown Road

22 Cedar $\mathrm{Cr}$ at Thixton Road

23 Pennsylvania Run at Mt. Washington Road

$24 \mathrm{Mill} \mathrm{Cr}$ Cutoff at Dover Road

25 Harrods $\mathrm{Cr}$ at Hunting $\mathrm{Cr}$ Drive

26 Long Run at State Highway 1531
Dissolved oxygen

$\begin{array}{rrrrr}65 & 372 & 15.4 & 3.7 & 22.8 \\ 63 & 29.4 & 15.7 & 7.6 & 54.7 \\ 55 & 263 & 16.6 & 2.6 & 22.3 \\ 72 & 105 & 28.9 & .4 & 4.4 \\ 61 & 85.8 & 12.1 & 8.2 & 31.4 \\ 64 & 124 & 24.5 & .8 & 6.2 \\ 70 & 99.0 & 24.0 & .6 & 6.0 \\ 70 & 18.2 & 28.3 & .1 & .7 \\ 74 & 35.8 & 16.0 & 2.5 & 13.3 \\ 68 & 86.5 & 11.2 & .1 & .3 \\ 74 & 58.9 & 12.4 & .3 & 1.5 \\ 69 & 71.7 & 23.3 & .6 & 2.5 \\ 63 & 19.0 & 17.8 & 2.4 & 13.4 \\ 37 & 665 & 18.8 & 3.3 & 18.0 \\ 69 & 91.6 & 17.7 & 3.8 & 21.6 \\ 65 & 33.5 & 12.1 & 3.2 & 13.6 \\ 71 & 128 & 19.4 & .4 & 2.0 \\ 63 & 32.0 & 28.9 & .3 & 3.5 \\ 59 & 49.8 & 23.4 & .5 & 4.4 \\ 54 & 1,060 & 17.9 & 9.4 & 41.9 \\ 64 & 85.2 & 14.1 & 5.9 & 35.1 \\ 63 & 34.7 & 16.9 & 1.7 & 15.1 \\ 50 & 17.2 & 20.0 & 7.0 & 36.4 \\ 55 & 612 & 21.8 & 1.3 & 8.1 \\ 34 & 97.2 & 13.0 & 2.7 & 23.7\end{array}$

Chemical oxygen demand, $0.25 \mathrm{~N}$ dicromate

1 Pond $\mathrm{Cr}$ at Pendleton Road

$2 \mathrm{Mill} \mathrm{Cr}$ at Orell Road

3 Pond $\mathrm{Cr}$ at Manslick Road

5 SF Beargrass $\mathrm{Cr}$ at Winter Avenue

8 MF Beargrass $\mathrm{Cr}$ at Beals Branch Road

9 Spring Ditch at Private Drive

10 Muddy Fork at Mockingbird Valley Road

11 Goose $\mathrm{Cr}$ at U.S. Highway 42

12 Little Goose $\mathrm{Cr}$ at U.S. Highway 42

13 Goose $\mathrm{Cr}$ at old Westport Road

14 Pope Lick at Pope Lick Road

15 Floyds Fork at former State Highway 155

16 Chenoweth Run at Gelhaus Road

17 Fern $\mathrm{Cr}$ at Old Bardstown Road

18 Northern Ditch at Preston Highway

19 Fishpool $\mathrm{Cr}$ at Bost Road

20 Southern Ditch at Minors Lane

21 Floyds Fork at Bardstown Road

22 Cedar $\mathrm{Cr}$ at Thixton Road

23 Pennsylvania Run at Mt. Washington Road

$24 \mathrm{Mi} 11 \mathrm{Cr}$ Cutoff at Dover Road

25 Harrods $\mathrm{Cr}$ at Hunting $\mathrm{Cr}$ Drive

26 Long Run at State Highway 1531

$\begin{array}{lcl}61 & 518 & 57 . \\ 60 & 55.0 & 57 . \\ 50 & 403 & 50 . \\ 67 & 124 & 66 . \\ 66 & 89.2 & 62 . \\ 65 & 58.3 & 71 . \\ 69 & 40.6 & 63 . \\ 63 & 109 & 68 . \\ 68 & 121 & 75 . \\ 64 & 96.2 & 59 . \\ 59 & 24.2 & 59 . \\ 34 & 698 & 51 . \\ 64 & 119 & 57 . \\ 61 & 34.8 & 61 . \\ 67 & 162 & 66 . \\ 58 & 43.5 & 60 . \\ 53 & 63.2 & 58 . \\ 50 & 1,100 & 65 . \\ 60 & 79.7 & 61 . \\ 60 & 62.6 & 51 . \\ 45 & 39.6 & 37 . \\ 52 & 659 & 56 . \\ 30 & 91.1 & 58.4\end{array}$

3.7

13.5

54.0

12.5

2.5

3. 2

1.5

.2

1.6

1. 5

9.3

13.7

16.1

9.8

1.0

2. 1

3.2

33.9

23.7

18.6

42.2

3. 6

7. 0

2.7

22.1

Biochemical oxygen demand, 5-day at $20^{\circ} \mathrm{C}$

1 Pond $\mathrm{Cr}$ at Pendleton Road

$2 \mathrm{Mill} \mathrm{Cr}$ at Orell Road

3 Pond $\mathrm{Cr}$ at Manslick Road

5 SF Beargrass $\mathrm{Cr}$ at Winter Avenue

6 SF Beargrass $\mathrm{Cr}$ at Trevilian Way

7 MF Beargrass $\mathrm{Cr}$ at Old Cannons Lane

8 MF Beargrass $\mathrm{Cr}$ at Beals Branch Road

9 Spring Ditch at Private Drive

10 Muddy Fork at Mockingbird Valley Road

11 Goose $\mathrm{Cr}$ at U.S. Highway 42

12 Little Goose $\mathrm{Cr}$ at U.S. Highway 42

13 Goose $\mathrm{Cr}$ at old Westport Road

14 Pope Lick at Pope Lick Road

15 Floyds Fork at former State Highway 155

16 Chenoweth Run at Gelhaus Road

17 Ferm $\mathrm{Cr}$ at O1d Bardstown Road

18 Northern Ditch at Preston Highway

$19 \mathrm{Fishpool} \mathrm{Cr}$ at Bost Road

20 Southern Ditch at Minors Lane

21 Floyds Fork at Bardstown Road

22 Cedar $\mathrm{Cr}$ at Thixton Road

23 Pennsylvania Run at Mt. Washington Road

$24 \mathrm{Mill} \mathrm{Cr}$ Cutoff at Dover Road

25 Harrods $\mathrm{Cr}$ at Hunting $\mathrm{Cr}$ Drive

26 Long Run at State Highway 1531

$\begin{array}{lcc}61 & 192 & 74.7 \\ 60 & 10.5 & 60.1 \\ 51 & 155 & 62.3 \\ 69 & 54.6 & 74.9 \\ 59 & 51.2 & 73.5 \\ 61 & 38.3 & 57.4 \\ 68 & 30.2 & 62.0 \\ 66 & 10.5 & 68.4 \\ 70 & 13.0 & 63.7 \\ 64 & 45.3 & 74.0 \\ 70 & 27.8 & 77.1 \\ 66 & 43.0 & 73.3 \\ 59 & 8.90 & 57.9 \\ 35 & 236 & 56.3 \\ 65 & 48.7 & 60.6 \\ 62 & 14.9 & 68.4 \\ 68 & 63.2 & 75.7 \\ 59 & 11.9 & 62.4 \\ 54 & 23.3 & 48.6 \\ 50 & 416 & 65.5 \\ 61 & 31.2 & 76.7 \\ 60 & 16.4 & 59.7 \\ 47 & 11.6 & 74.5 \\ 52 & 262 & 74.2 \\ 30 & 37.4 & 70.3 \\ & & \end{array}$

3.7

17.2

39.6

23.9

4.4

22.5

2.9

2. 7

1. 1

8. 4

1.2

1.6

10.5

12. 5

16.9

13.8

1.6

1. 8

33.

33.4
24.6

24.6
24.3

29.8

7.0

17.7 
Table 15. Estimates of mean annual loads of constituents in base flow in urban watersheds of Jefferson County, Kentucky, 1988-92--Continued

[Cr, Creek; SF, South Fork; MF, Middle Fork; ${ }^{\circ} \mathrm{C}$, degrees Celsius ]

\begin{tabular}{|c|c|c|c|c|c|}
\hline Site number and name & $\begin{array}{c}\text { Number } \\
\text { of } \\
\text { obser- } \\
\text { vations }\end{array}$ & $\begin{array}{c}\text { Mean } \\
\text { annual } \\
\text { load, } \\
\text { in tons }\end{array}$ & $\begin{array}{l}\text { Standard } \\
\text { error of } \\
\text { regression }\end{array}$ & $\begin{array}{c}\text { Flow duration } \\
\text { of greatest } \\
\text { sampled } \\
\text { discharge, } \\
\text { in percent }\end{array}$ & $\begin{array}{l}\text { Percentage of } \\
\text { load estimated } \\
\text { beyond range } \\
\text { of sampled } \\
\text { discharge }\end{array}$ \\
\hline
\end{tabular}

1 Pond $\mathrm{Cr}$ at Pendleton Road

$2 \mathrm{Mill} \mathrm{Cr}$ at Orell Road

3 Pond $\mathrm{Cr}$ at Manslick Road

5 SF Beargrass $C r$ at Winter Avenue

6 SF Beargrass $\mathrm{Cr}$ at Trevilian Way

9 Spring Ditch at Private Drive

10 Muddy Fork at Mockingbird Valley Road

11 Goose $\mathrm{Cr}$ at U.S. Highway 42

12 Little Goose $\mathrm{Cr}$ at U.S. Highway 42

13 Goose $\mathrm{Cr}$ at old Westport Road

14 Pope Lick at Pope Lick Road

15 Floyds Fork at former State Highway 155

16 Chenoweth Run at Gelhaus Road

17 Fern $\mathrm{Cr}$ at Old Bardstown Road

18 Northern Ditch at Preston Highway

21 Floyds Fork at Bardstown Road

22 Cedar $\mathrm{Cr}$ at Thixton Road

23 Pennsylvania Run at Mt. Washington Road

25 Harrods $\mathrm{Cr}$ at Hunting $\mathrm{Cr}$ Drive

\section{Calcium, total as $\mathrm{Ca}$}

\begin{tabular}{|c|c|c|c|c|}
\hline $\begin{array}{l}15 \\
14 \\
13 \\
14 \\
12 \\
13 \\
12 \\
14 \\
13 \\
14 \\
15 \\
10 \\
16 \\
12 \\
12 \\
10 \\
12 \\
13 \\
10\end{array}$ & $\begin{array}{r}2,170 \\
108 \\
1,620 \\
765 \\
605 \\
123 \\
384 \\
458 \\
320 \\
344 \\
116 \\
3,860 \\
472 \\
192 \\
614 \\
6,180 \\
586 \\
162 \\
3,010\end{array}$ & $\begin{array}{r}14.6 \\
41.0 \\
11.8 \\
22.2 \\
10.7 \\
15.7 \\
8.8 \\
11.8 \\
12.9 \\
13.7 \\
9.4 \\
10.1 \\
20.2 \\
8.1 \\
7.7 \\
17.9 \\
28.7 \\
28.8 \\
10.1\end{array}$ & $\begin{array}{r}5.9 \\
10.6 \\
2.6 \\
4.4 \\
8.2 \\
3.7 \\
11.0 \\
19.6 \\
.3 \\
8.2 \\
8.7 \\
11.9 \\
5.9 \\
4.5 \\
.4 \\
25.8 \\
5.9 \\
1.7 \\
8.6\end{array}$ & $\begin{array}{r}23.9 \\
43.7 \\
19.4 \\
27.1 \\
31.3 \\
13.4 \\
47.0 \\
47.5 \\
1.3 \\
23.1 \\
32.0 \\
43.2 \\
28.1 \\
17.2 \\
1.8 \\
69.7 \\
39.2 \\
16.4 \\
30.6\end{array}$ \\
\hline
\end{tabular}

1 Pond $\mathrm{Cr}$ at Pendleton Road

$2 \mathrm{Mill} \mathrm{Cr}$ at Orell Road

3 Pond $\mathrm{Cr}$ at Manslick Road

$5 \mathrm{SF}$ Beargrass $\mathrm{Cr}$ at Winter Avenue

6 SF Beargrass $C_{r}$ at Trevilian Way

9 Spring Ditch at Private Drive

10 Muddy Fork at Mockingbird Valley Road

11 Goose $\mathrm{Cr}$ at U.S. Highway 42

12 Little Goose $C_{r}$ at U.S. Highway 42

13 Goose $\mathrm{Cr}$ at old Westport Road

14 Pope Lick at Pope Lick Road

15 Floyds Fork at former State Highway 155

16 Chenoweth Run at Gelhaus Road

17 Fern $\mathrm{Cr}$ at Old Bardstown Road

18 Northern Ditch at Preston Highway

21 Floyds Fork at Bardstown Road

22 Cedar $\mathrm{Cr}$ at Thixton Road

23 Pennsylvania Run at Mt. Washington Road

Magnesium, total as $\mathrm{Mg}$

25 Harrods $\mathrm{Cr}$ at Hunting $\mathrm{Cr}$ Drive

$\begin{array}{lcr}15 & 800 & 15.9 \\ 14 & 38.1 & 49.5 \\ 13 & 629 & 9.8 \\ 14 & 164 & 20.4 \\ 12 & 143 & 7.9 \\ 13 & 32.2 & 16.3 \\ 12 & 51.9 & 14.4 \\ 14 & 193 & 12.3 \\ 13 & 110 & 12.5 \\ 14 & 144 & 12.4 \\ 15 & 50.0 & 9.8 \\ 10 & 966 & 12.7 \\ 15 & 177 & 15.9 \\ 12 & 84.1 & 10.2 \\ 12 & 242 & 12.7 \\ 10 & 2,290 & 15.4 \\ 12 & 302 & 36.1 \\ 13 & 80.4 & 21.6 \\ 10 & 1,380 & 10.6\end{array}$

$\begin{array}{rr}5.9 & 23.7 \\ 10.6 & 42.5 \\ 2.6 & 19.7 \\ 4.4 & 27.3 \\ 8.2 & 30.4 \\ 3.7 & 14.4 \\ 11.0 & 40.5 \\ 19.6 & 49.2 \\ .3 & 1.3 \\ 8.2 & 23.7 \\ 8.7 & 34.5 \\ 11.9 & 39.8 \\ 5.9 & 32.4 \\ 4.5 & 17.7 \\ .4 & 2.0 \\ 25.8 & 74.5 \\ 5.9 & 44.4 \\ 1.7 & 18.0 \\ 8.6 & 27.9\end{array}$

Alkalinity, titration to 4.5 , as $\mathrm{CaCO}_{3}$

1 Pond $\mathrm{Cr}$ at Pendleton Road

$2 \mathrm{Mill} \mathrm{Cr}$ at Orell Road

3 Pond $\mathrm{Cr}$ at Manslick Road

5 SF Beargrass $\mathrm{Cr}$ at Winter Avenue

$6 \mathrm{SF}$ Beargrass $\mathrm{Cr}$ at Trevilian Way

7 MF Beargrass $\mathrm{Cr}$ at Old Cannons Lane

8 MF Beargrass $C_{r}$ at Beals Branch Road

9 Spring Ditch at Private Drive

10 Muddy Fork at Mockingbird Valley Road

11 Goose $\mathrm{Cr}$ at U.S. Highway 42

12 Little Goose $\mathrm{Cr}_{r}$ at U.S. Highway 42

13 Goose $\mathrm{Cr}$ at old Westport Road

14 Pope Lick at Pope Lick Road

15 Floyds Fork at former State Highway 155

16 Chenoweth Run at Gelhaus Road

17 Fern $\mathrm{Cr}$ at Old Bardstown Road

18 Northern Ditch at Preston Highway

$19 \mathrm{Fishpool} \mathrm{Cr}$ at Bost Road

20 Southern Ditch at Minors Lane

21 Floyds Fork at Bardstown Road

22 Cedar $\mathrm{Cr}$ at Thixton Road

23 Pennsylvania Run at Mt. Washington Road

$24 \mathrm{Mill} \mathrm{Cr}$ Cutoff at Dover Road

25 Harrods $\mathrm{Cr}$ at funting $\mathrm{Cr}$ Drive

26 Long Run at State Highway 1531

$\begin{array}{rrr}63 & 6,060 & 25.3 \\ 61 & 603 & 42.8 \\ 52 & 5,270 & 16.2 \\ 68 & 2,010 & 22.1 \\ 58 & 1,860 & 20.4 \\ 61 & 2,090 & 18.5 \\ 68 & 2,030 & 14.1 \\ 66 & 313 & 29.7 \\ 70 & 851 & 21.7 \\ 65 & 1,560 & 14.8 \\ 70 & 1,060 & 13.1 \\ 66 & 1,160 & 19.9 \\ 59 & 417 & 15.1 \\ 35 & 10,700 & 28.0 \\ 65 & 1,510 & 17.2 \\ 62 & 734 & 27.7 \\ 68 & 2,110 & 13.5 \\ 59 & 502 & 16.6 \\ 54 & 807 & 18.4 \\ 50 & 21,000 & 40.3 \\ 61 & 1,600 & 39.7 \\ 60 & 570 & 22.0 \\ 46 & 233 & 27.3 \\ 52 & 11,500 & 14.7 \\ 30 & 1,730 & 16.5 \\ & & \end{array}$

$\begin{array}{rr}3.7 & 20.2 \\ 7.6 & 60.6 \\ 2.6 & 23.8 \\ .4 & 4.4 \\ 8.2 & 40.6 \\ .8 & 5.8 \\ .6 & 5.1 \\ .1 & .4 \\ 2.5 & 14.2 \\ .1 & .3 \\ .3 & 1.4 \\ .6 & 2.5 \\ 2.4 & 12.9 \\ 3.3 & 15.8 \\ 3.8 & 24.6 \\ 3.2 & 18.0 \\ .4 & 2.1 \\ .3 & 3.2 \\ .7 & 5.8 \\ 9.4 & 43.4 \\ 5.9 & 40.0 \\ 1.7 & 17.6 \\ 7.2 & 36.1 \\ 1.3 & 6.8 \\ 2.7 & 25.0\end{array}$


Table 15. Estimates of mean annual loads of constituents in base flow in urban watersheds of Jefferson County, Kentucky, 1988-92--Continued

[Cr, Creek; SF, South Fork; MF, Middle Fork; ${ }^{\circ} \mathrm{C}$, degrees Celsius]

\begin{tabular}{|c|c|c|c|c|c|}
\hline Site number and name & $\begin{array}{c}\text { Number } \\
\text { of } \\
\text { obser- } \\
\text { vations }\end{array}$ & $\begin{array}{l}\text { Mean } \\
\text { annual } \\
\text { load, } \\
\text { in tons }\end{array}$ & $\begin{array}{l}\text { Standard } \\
\text { error of } \\
\text { regression }\end{array}$ & $\begin{array}{c}\text { Flow duration } \\
\text { of greatest } \\
\text { sampled } \\
\text { discharge, } \\
\text { in percent }\end{array}$ & $\begin{array}{l}\text { Percentage of } \\
\text { load estimated } \\
\text { beyond range } \\
\text { of sampled } \\
\text { discharge }\end{array}$ \\
\hline
\end{tabular}

Dissolved solids, residue at $105^{\circ} \mathrm{C}$

1 Pond $\mathrm{Cr}$ at Pendleton Road

$2 \mathrm{Mill} \mathrm{Cr}$ at Orell Road

3 Pond $\mathrm{Cr}$ at Manslick Road

5 SF Beargrass $\mathrm{Cr}$ at Winter Avenue

$6 \mathrm{SF}$ Beargrass $\mathrm{Cr}$ at Trevilian Way

7 MF Beargrass $\mathrm{Cr}$ at Old Cannons Lane

$8 \mathrm{MF}$ Beargrass $\mathrm{Cr}$ at Beals Branch Road

9 Spring Ditch at Private Drive

10 Muddy Fork at Mockingbird Valley Road

11 Goose $\mathrm{Cr}$ at U.S. Highway 42

12 Little Goose $\mathrm{Cr}$ at U.S. Highway 42

13 Goose $\mathrm{Cr}$ at old Westport Road

14 Pope Lick at Pope Lick Road

15 Floyds Fork at former State Highway 155

16 Chenoweth Run at Gelhaus Road

$17 \mathrm{Fern} \mathrm{Cr}$ at Old Bardstown Road

18 Northern Ditch at Preston Highway

$19 \mathrm{Fishpool} \mathrm{Cr}$ at Bost Road

20 Southern Ditch at Minors Lane

21 Floyds Fork at Bardstown Road

22 Cedar $\mathrm{Cr}$ at Thixton Road

23 Pennsylvania Run at Mt. Washington Road

$24 \mathrm{Mi} 11 \mathrm{Cr}$ Cutoff at Dover Road

25 Harrods $\mathrm{Cr}$ at Hunting $\mathrm{Cr}$ Drive

26 Long Run at State Highway 1531

$\begin{array}{rrr}63 & 13,300 & 22.4 \\ 61 & 838 & 47.4 \\ 52 & 11,200 & 20.7 \\ 69 & 3,690 & 35.6 \\ 59 & 3,160 & 13.2 \\ 61 & 3,440 & 16.2 \\ 68 & 3,430 & 18.2 \\ 66 & 669 & 15.2 \\ 70 & 1,700 & 14.4 \\ 65 & 2,520 & 11.6 \\ 70 & 1,790 & 11.1 \\ 66 & 2,050 & 32.6 \\ 58 & 694 & 13.1 \\ 35 & 17,400 & 13.0 \\ 65 & 2,780 & 10.3 \\ 61 & 1,260 & 12.4 \\ 67 & 4,030 & 23.8 \\ 59 & 1,090 & 18.5 \\ 53 & 1,840 & 12.6 \\ 50 & 28,300 & 14.9 \\ 60 & 2,770 & 24.3 \\ 60 & 1,290 & 32.0 \\ 47 & 538 & 20.6 \\ 52 & 16,200 & 14.0 \\ 30 & 2,450 & 14.6\end{array}$

$\begin{array}{rr}3.7 & 16.0 \\ 7.6 & 48.0 \\ 2.6 & 16.4 \\ .4 & 3.1 \\ 8.2 & 31.7 \\ .8 & 5.4 \\ .6 & 4.9 \\ .1 & .5 \\ 2.5 & 12.7 \\ .1 & .2 \\ .3 & 1.2 \\ .6 & 1.9 \\ 2.4 & 9.6 \\ 3.3 & 15.8 \\ 3.8 & 17.0 \\ 3.2 & 11.2 \\ .4 & 1.3 \\ .3 & 2.9 \\ .7 & 5.4 \\ 9.4 & 36.4 \\ 5.9 & 25.2 \\ 1.7 & 17.4 \\ 7.0 & 39.5 \\ 1.3 & 4.9 \\ 2.7 & 24.1\end{array}$

4

31.7

4

2.7

1.2

1.9

15.8

1. 3

4

6.4

17.4

4.9

24.1

Suspended solids, residue at $105^{\circ} \mathrm{C}$

1 Pond $\mathrm{Cr}$ at Pendleton Road

3 Pond $\mathrm{Cr}$ at Manslick Road

5 SF Beargrass $\mathrm{Cr}$ at Winter Avenue

$6 \mathrm{SF}$ Beargrass $\mathrm{Cr}$ at Trevilian Way

7 MF Beargrass $\mathrm{Cr}$ at Old Cannons Lane

$8 \mathrm{MF}$ Beargrass $\mathrm{Cr}$ at Beals Branch Road

9 Spring Ditch at Private Drive

10 Muddy Fork at Mockingbird Valley Road

11 Goose $\mathrm{Cr}$ at U.S. Highway 42

12 Little Goose $\mathrm{Cr}$ at U.S. Highway 42

13 Goose $\mathrm{Cr}$ at old Westport Road

14 Pope Lick at Pope Lick Road

16 Chenoweth Run at Gelhaus Road

17 Fern $\mathrm{Cr}$ at Old Bardstown Road

18 Northern Ditch at Preston Highway

$19 \mathrm{Fishpoo1} \mathrm{Cr}$ at Bost Road

20 Southern Ditch at Minors Lane

21 Floyds Fork at Bardstown Road

22 Cedar $\mathrm{Cr}$ at Thixton Road

23 Pennsylvania Run at Mt. Washington Road

$24 \mathrm{Mi} 11 \mathrm{Cr}$ Cutoff at Dover Road

26 Long Run at State Highway 1531
$2 \mathrm{Mill} \mathrm{Cr}$ at Orell Road

15 Floyds Fork at former State Highway 155

25 Harrods $\mathrm{Cr}$ at Hunting $\mathrm{Cr}$ Drive

$\begin{array}{rrr}63 & 1,000 & 78.1 \\ 61 & 27.7 & 73.7 \\ 52 & 1,100 & 53.1 \\ 68 & 228 & 78.9 \\ 59 & 148 & 71.8 \\ 61 & 66.6 & 72.1 \\ 68 & 96.4 & 91.3 \\ 66 & 45.6 & 93.8 \\ 70 & 90.0 & 93.2 \\ 65 & 80.8 & 70.7 \\ 70 & 96.5 & 82.7 \\ 66 & 157 & 98.6 \\ 59 & 26.2 & 86.3 \\ 35 & 662 & 85.3 \\ 65 & 75.9 & 84.5 \\ 61 & 56.5 & 81.6 \\ 68 & 124 & 79.6 \\ 59 & 46.6 & 89.7 \\ 53 & 79.5 & 73.0 \\ 50 & 1,230 & 71.6 \\ 60 & 47.2 & 88.5 \\ 60 & 64.1 & 105 \\ 47 & 16.9 & 74.3 \\ 52 & 1,200 & 72.6 \\ 30 & 110 & 90.7\end{array}$

Residue, volatile nonfilterable

1 Pond $\mathrm{Cr}$ at Pendleton Road

$2 \mathrm{Mill} \mathrm{Cr}$ at Orell Road

3 Pond $\mathrm{Cr}$ at Manslick Road

$5 \mathrm{SF}$ Beargrass $\mathrm{Cr}$ at Winter Avenue

6 SF Beargrass $\mathrm{Cr}$ at Trevilian Way

7 MF Beargrass $\mathrm{Cr}$ at O1d Cannons Lane

8 MF Beargrass $\mathrm{Cr}$ at Beals Branch Road

9 Spring Ditch at Private Drive

10 Muddy Fork at Mockingbird Valley Road

11 Goose $\mathrm{Cr}$ at U.S. Highway 42

12 Little Goose $\mathrm{Cr}$ at U.S. Highway 42

13 Goose $\mathrm{Cr}$ at old Westport Road

14 Pope Lick at Pope Lick Road

15 Floyds Fork at former State Highway 155

16 Chenoweth Run at Gelhaus Road

17 Fern $\mathrm{Cr}$ at Old Bardstown Road

18 Northern Ditch at Preston Highway

$19 \mathrm{Fishpool} \mathrm{Cr}$ at Bost Road

20 Southern Ditch at Minors Lane

21 Floyds Fork at Bardstown Road

22 Cedar $\mathrm{Cr}$ at Thixton Road

23 Pennsylvania Run at Mt. Washington Road

$24 \mathrm{Mill} \mathrm{Cr}$ Cutoff at Dover Road

25 Harrods $\mathrm{Cr}$ at Hunting $\mathrm{Cr}$ Drive

26 Long Run at State Highway 1531

$\begin{array}{lcc}63 & 179 & 83.8 \\ 61 & 20.8 & 134 \\ 52 & 233 & 103 \\ 68 & 158 & 127 \\ 59 & 62.4 & 99.7 \\ 61 & 85.6 & 109 \\ 68 & 35.7 & 93.7 \\ 66 & 16.1 & 94.8 \\ 70 & 25.1 & 99.1 \\ 65 & 39.4 & 127 \\ 70 & 60.9 & 125 \\ 66 & 66.7 & 129 \\ 59 & 13.2 & 100 \\ 35 & 202 & 106 \\ 65 & 36.5 & 167 \\ 61 & 23.7 & 100 \\ 68 & 65.1 & 117 \\ 59 & 20.9 & 128 \\ 53 & 32.3 & 109 \\ 50 & 548 & 101 \\ 60 & 38.0 & 130 \\ 60 & 20.7 & 124 \\ 47 & 6.40 & 91.4 \\ 52 & 526 & 125 \\ 30 & 27.2 & 96.0\end{array}$

3.7

7.6

2.6

8.2

8.2
.8

.6

.1
2.5
.1

3
6

2.4
3.3

3. 8

3. 2

.4

.3

9.4

5.9

1. 7

7.0

1. 3

2. 7
29.4

27.3

21.4

29.7

4.1
3.7

.9

11. 1

1. 5

2.0

13. 0

12. 1

23.3

17.4

1.8

2. 8

6.7

40.4

14.1

8.0

42.7

3.0
21.9

$\begin{array}{rr}3.7 & 13.5 \\ 7.6 & 47.7 \\ 2.6 & 11.5 \\ .4 & 3.9 \\ 8.2 & 32.8 \\ .8 & 5.0 \\ .6 & 3.1 \\ .1 & .5 \\ 2.5 & 9.9 \\ .1 & .2 \\ .3 & 1.3 \\ .6 & 1.9 \\ 2.4 & 9.5 \\ 3.3 & 12.5 \\ 3.8 & 14.3 \\ 3.2 & 16.6 \\ .4 & 1.7 \\ .3 & 3.0 \\ .7 & 4.8 \\ 9.4 & 46.6 \\ 5.9 & 28.9 \\ 1.7 & 12.3 \\ 7.0 & 34.9 \\ 1.3 & 3.9 \\ 2.7 & 18.1\end{array}$

13.5 
Table 15. Estimates of mean annual loads of constituents in base flow in urban watersheds of Jefferson County, Kentucky, 1988-92--Continued

[Cr, Creek; SF, South Fork; MF, Middle Fork; ${ }^{\circ} \mathrm{C}$, degrees Celsius]

\begin{tabular}{|c|c|c|c|c|c|}
\hline Site number and name & $\begin{array}{c}\text { Number } \\
\text { of } \\
\text { obser- } \\
\text { vations }\end{array}$ & $\begin{array}{l}\text { Mean } \\
\text { annual } \\
\text { load, } \\
\text { in tons }\end{array}$ & $\begin{array}{l}\text { Standard } \\
\text { error of } \\
\text { regression }\end{array}$ & $\begin{array}{l}\text { Flow duration } \\
\text { of greatest } \\
\text { sampled } \\
\text { discharge, } \\
\text { in percent }\end{array}$ & $\begin{array}{l}\text { Percentage of } \\
\text { load estimated } \\
\text { beyond range } \\
\text { of sampled } \\
\text { discharge }\end{array}$ \\
\hline
\end{tabular}

Suspended solids, nonvolatile

$\begin{array}{lrrr}1 \text { Pond Cr at Pendleton Road } & 16 & 537 & 123 \\ 2 \text { Mill Cr at Orell Road } & 16 & 110 & 60.8 \\ 3 \text { Pond Cr at Manslick Road } & 14 & 851 & 78.3 \\ 5 \text { SF Beargrass Cr at Winter Avenue } & 15 & 120 & 316 \\ 7 \text { MF Beargrass Cr at Old Cannons Lane } & 13 & 24.1 & 66.6 \\ 8 \text { MF Beargrass Cr at Beals Branch Road } & 16 & 30.9 & 54.9 \\ 9 \text { Spring Ditch at Private Drive } & 15 & 10.0 & 52.8 \\ 10 \text { Muddy Fork at Mockingbird Valley Road } & 13 & 23.9 & 101 \\ 11 \text { Goose Cr at U.S. Highway 42 } & 13 & 27.0 & 79.3 \\ 12 \text { Little Goose Cr at U.S. Highway 42 } & 15 & 39.7 & 81.8 \\ 13 \text { Goose Cr at old Westport Road } & 13 & 148 & 88.4 \\ 14 \text { Pope Lick at Pope Lick Road } & 13 & 104 & 287 \\ 16 \text { Chenoweth Run at Gelhaus Road } & 15 & 1,440 & 274 \\ 17 \text { Fern Cr at Old Bardstown Road } & 15 & 21.4 & 78.1 \\ 18 \text { Northern Ditch at Preston Highway } & 14 & 72.3 & 51.8 \\ 19 \text { Fishpool Cr at Bost Road } & 12 & 22.5 & 52.8 \\ 20 \text { Southerm Ditch at Minors Lane } & 12 & 23.8 & 63.7 \\ 21 \text { Floyds Fork at Bardstown Road } & 10 & 711 & 48.2 \\ 23 \text { Pennsylvania Run at Mt. Washington Road } & 13 & 15.7 & 82.8 \\ 24 \text { Mill Cr Cutoff at Dover Road } & 14 & 18.6 & 60.4 \\ 25 \text { Harrods Cr at Hunting Cr Drive } & 11 & 554 & 74.4\end{array}$

Nitrogen, nitrate, total as $\mathrm{N}$

1 Pond $\mathrm{Cr}$ at Pendleton Road

$2 \mathrm{Mill} \mathrm{Cr}$ at Orell Road

3 Pond $\mathrm{Cr}$ at Manslick Road

5 SF Beargrass $\mathrm{Cr}$ at Winter Avenue

6 SF Beargrass $\mathrm{Cr}$ at Trevilian Way

7 MF Beargrass $\mathrm{Cr}$ at Old Cannons Lane

$8 \mathrm{MF}$ Beargrass $\mathrm{Cr}$ at Beals Branch Road

9 Spring Ditch at Private Drive

10 Muddy Fork at Mockingbird Valley Road

11 Goose $C_{I}$ at U.S. Highway 42

12 Little Goose $\mathrm{Cr}$ at U.S. Highway 42

13 Goose $\mathrm{Cr}$ at old Westport Road

14 Pope Lick at Pope Lick Road

15 Floyds Fork at former State Highway 155

16 Chenoweth Run at Gelhaus Road

17 Fern $\mathrm{Cr}$ at Old Bardstown Road

18 Northern Ditch at Preston Highway

19 Fishpool $\mathrm{Cr}$ at Bost Road

20 Southern Ditch at Minors Lane

21 Floyds Fork at Bardstown Road

22 Cedar $\mathrm{Cr}$ at Thixton Road

23 Pennsylvania Run at Mt. Washington Road

$24 \mathrm{Mill} \mathrm{Cr}$ Cutoff at Dover Road

25 Harrods $\mathrm{Cr}$ at Hunting $\mathrm{Cr}$ Drive

26 Long Run at State Highway 1531

$\begin{array}{lcr}63 & 78.4 & 82.8 \\ 61 & 2.38 & 110 \\ 52 & 55.5 & 92.8 \\ 68 & 25.5 & 75.3 \\ 58 & 16.1 & 86.1 \\ 61 & 23.8 & 88.4 \\ 68 & 22.4 & 82.1 \\ 66 & 4.02 & 95.8 \\ 70 & 12.8 & 89.3 \\ 64 & 27.6 & 50.7 \\ 68 & 24.2 & 48.5 \\ 65 & 20.3 & 66.2 \\ 59 & 5.08 & 91.0 \\ 35 & 49.7 & 113 \\ 64 & 26.0 & 79.2 \\ 61 & 13.9 & 87.9 \\ 68 & 36.6 & 111 \\ 59 & 8.82 & 104 \\ 54 & 12.7 & 97.8 \\ 50 & 111 & 65.1 \\ 61 & 18.1 & 63.0 \\ 60 & 9.84 & 84.1 \\ 46 & 3.66 & 90.0 \\ 51 & 115 & 49.2 \\ 30 & 7.98 & 118\end{array}$

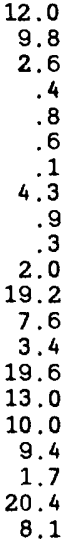

44.2

69.1

24.4

4. 0

5.1

20.3

2. 1

1.4

1.7

90.4

5.5

16.0

27.9

64.6

31.7

44.3

10.8

87.5

23.9

$\begin{array}{rr}3.7 & 19.1 \\ 7.6 & 38.5 \\ 2.6 & 13.4 \\ .4 & 7.2 \\ 8.2 & 35.7 \\ .8 & 6.2 \\ .6 & 6.1 \\ .1 & .7 \\ 2.5 & 6.0 \\ .1 & .3 \\ .3 & 1.5 \\ .6 & 2.5 \\ 2.4 & 6.5 \\ 3.3 & 13.8 \\ 3.8 & 10.7 \\ 3.2 & 10.2 \\ .4 & .8 \\ .3 & 2.3 \\ .7 & 6.2 \\ 9.4 & 36.6 \\ 5.9 & 18.9 \\ 1.7 & 11.2 \\ 7.0 & 37.0 \\ 1.3 & 8.8 \\ 2.7 & 22.8\end{array}$

Nitrogen, nitrite, total as $\mathrm{N}$

1 Pond $\mathrm{Cr}$ at Pendleton Road

3 Pond $\mathrm{Cr}$ at Manslick Road

5 SF Beargrass $C_{I}$ at Winter Avenue

6 SF Beargrass $\mathrm{Cr}$ at Trevilian Way

7 MF Beargrass $\mathrm{Cr}$ at Old Cannons Lane

$8 \mathrm{MF}$ Beargrass $\mathrm{Cr}$ at Beals Branch Road

9 Spring Ditch at Private Drive

10 Muddy Fork at Mockingbird Valley Road

11 Goose $\mathrm{Cr}$ at U.S. Highway 42

12 Little Goose $\mathrm{Cr}$ at U.S. Highway 42

13 Goose $\mathrm{Cr}$ at old Westport Road

14 Pope Lick at Pope Lick Road

15 Floyds Fork at former State Highway 155

16 Chenoweth Run at Gelhaus Road

17 Fern $\mathrm{Cr}$ at Old Bardstown Road

18 Northern Ditch at Preston Highway

$19 \mathrm{Fishpool} \mathrm{Cr}$ at Bost Road

20 Southerm Ditch at Minors Lane

21 Floyds Fork at Bardstown Road

22 Cedar $\mathrm{Cr}$ at Thixton Road

23 Pennsylvania Run at Mt. Washington Road

24 Mill Cr Cutoff at Dover Road

25 Harrods $\mathrm{Cr}$ at Hunting $\mathrm{Cr}$ Drive

$\begin{array}{rrr}63 & 2.86 & 71.5 \\ 52 & 2.60 & 57.4 \\ 69 & .46 & 58.1 \\ 59 & .28 & 48.7 \\ 61 & .16 & 78.4 \\ 68 & .16 & 62.4 \\ 66 & .08 & 57.6 \\ 70 & .20 & 73.5 \\ 65 & .40 & 74.9 \\ 70 & .24 & 83.1 \\ 66 & .64 & 110 \\ 59 & .16 & 82.7 \\ 35 & 1.18 & 80.5 \\ 65 & .76 & 117 \\ 62 & .20 & 89.1 \\ 68 & .68 & 56.7 \\ 59 & .32 & 111 \\ 54 & .36 & 70.6 \\ 50 & 1.62 & 66.5 \\ 61 & .24 & 79.9 \\ 60 & .42 & 81.9 \\ 47 & .30 & 104 \\ 52 & 2.64 & 98.7\end{array}$

$\begin{array}{rr}3.7 & 26.6 \\ 2.6 & 20.3 \\ .4 & 2.1 \\ 8.2 & 20.0 \\ .8 & 2.8 \\ .6 & 2.4 \\ .1 & .5 \\ 2.5 & 11.8 \\ .1 & .4 \\ .3 & 1.5 \\ .6 & 3.0 \\ 2.4 & 8.3 \\ 3.3 & 14.8 \\ 3.8 & 14.5 \\ 3.2 & 12.0 \\ .4 & .8 \\ .3 & 2.1 \\ .7 & 5.0 \\ 9.4 & 38.1 \\ 5.9 & 19.4 \\ 1.7 & 16.9 \\ 7.0 & 53.9 \\ 1.3 & 7.0\end{array}$


Table 15. Estimates of mean annual loads of constituents in base flow in urban watersheds of Jefferson County, Kentucky, 1988-92--Continued

[Cr, Creek; SF, South Fork; M, Middle Fork; 'C, degrees Celsius]

\begin{tabular}{|c|c|c|c|c|c|}
\hline Site number and name & $\begin{array}{c}\text { Number } \\
\text { of } \\
\text { obser- } \\
\text { vations }\end{array}$ & $\begin{array}{l}\text { Mean } \\
\text { annual } \\
\text { load, } \\
\text { in tons }\end{array}$ & $\begin{array}{l}\text { Standard } \\
\text { error of } \\
\text { regression }\end{array}$ & $\begin{array}{c}\text { Flow duration } \\
\text { of greatest } \\
\text { sampled } \\
\text { discharse, } \\
\text { in percent }\end{array}$ & $\begin{array}{l}\text { Percentage of } \\
\text { load estimated } \\
\text { beyond range } \\
\text { of sampled } \\
\text { discharge }\end{array}$ \\
\hline
\end{tabular}

1 Pond $\mathrm{Cr}$ at Pendleton Road

$2 \mathrm{Mill} \mathrm{Cr}$ at Orell Road

3 Pond $\mathrm{Cr}$ at Manslick Road

5 SF Beargrass $\mathrm{Cr}$ at Winter Avenue

6 SF Beargrass $\mathrm{Cr}$ at Trevilian Way

7 MF Beargrass $\mathrm{Cr}$ at Old Cannons Lane

8 MF Beargrass $\mathrm{Cr}$ at Beals Branch Road

9 Spring Ditch at Private Drive

10 Muddy Fork at Mockingbird Valley Road

11 Goose $\mathrm{Cr}$ at U.S. Highway 42

12 Little Goose $\mathrm{Cr}$ at U.S. Highway 42

14 Pope Lick at Pope Lick Road

17 Fern $\mathrm{Cr}$ at Old Bardstown Road

18 Northern Ditch at Preston Highway

19 Fishpool $\mathrm{Cr}$ at Bost Road

20 Southern Ditch at Minors Lane

21 Floyds Fork at Bardstown Road

22 Cedar $\mathrm{Cr}$ at Thixton Road

23 Pennsylvania Run at Mt. Washington Road

Nitrogen, ammonia, total as

$24 \mathrm{Mill} \mathrm{Cr}$ Cutoff at Dover Road

$\begin{array}{lcc}61 & 8.48 & 173 \\ 59 & .10 & 149 \\ 51 & 15.5 & 198 \\ 67 & 2.98 & 193 \\ 58 & 3.00 & 184 \\ 59 & .48 & 156 \\ 66 & .86 & 165 \\ 65 & .34 & 197 \\ 69 & .24 & 158 \\ 65 & .98 & 178 \\ 70 & .44 & 161 \\ 59 & .40 & 167 \\ 60 & .70 & 190 \\ 68 & 3.08 & 215 \\ 59 & .30 & 177 \\ 54 & .72 & 185 \\ 50 & 11.7 & 173 \\ 60 & 1.02 & 179 \\ 58 & .56 & 177 \\ 46 & .78 & 160\end{array}$

\section{Nitrogen, organic, dissolved as $\mathrm{N}$}

1 Pond $\mathrm{Cr}$ at Pendleton Road
2 Mill $\mathrm{Cr}$ at Orell Road
3 Pond Cr at Manslick Road
5 SF Beargrass $\mathrm{Cr}$ at Winter Avenue
6 SF Beargrass $\mathrm{Cr}$ at Trevilian Way
7 MF Beargrass Cr at Old Cannons Lane
8 MF Beargrass Cr at Beals Branch Road
9 Spring Ditch at Private Drive
10 Muddy Fork at Mockingbird Valley Road
11 Goose Cr at U.S. Highway 42
12 Little Goose Cr at U.S. Highway 42
13 Goose Cr at old Westport Road
14 Pope Lick at Pope Lick Road
15 Floyds Fork at former State Highway 155
16 Chenoweth Run at Gelhaus Road
17 Fern Cr at Old Bardstown Road
18 Northerm Ditch at Preston Highway
19 Fishpool Cr at Bost Road
20 Southern Ditch at Minors Lane
21 Floyds Fork at Bardstown Road
22 Cedar Cr at Thixton Road
23 Pennsylvania Run at Mt. Washington Road
24 Mill Cr Cutoff at Dover Road
25 Harrods Cr at Hunting Cr Drive
26 Long Run at State Highway 1531

1 Pond $\mathrm{Cr}$ at Pendleton Road

$2 \mathrm{Mill} \mathrm{Cr}$ at Orell Road

7 MF Beargrass $\mathrm{Cr}$ at Old Cannons Lan

8 MF Beargrass $\mathrm{Cr}$ at Beals Branch Road

9 Spring Ditch at Private Drive

11 Goose $\mathrm{Cr}$ at U.S. Highway 42

12 Little Goose $\mathrm{Cr}$ at U.S. Highway 42

13 Goose $\mathrm{Cr}$ at old Westport Road

15 Floyds Fork at former State Highway 155

16 Chenoweth Run at Gelhaus Road

17 Fern $\mathrm{Cr}$ at Old Bardstown Road

$19 \mathrm{Fishpol} \mathrm{Cr}$ at Bost Road

20 Southern Ditch at Minors Lane

21 Floyds Fork at Bardstown Road

2edar Cr at Thixton Road

$24 \mathrm{Mill}$ Cr Cutoff at Dover Road

26 Long Run at State Highway 1531

$\begin{array}{lcc}62 & 22.4 & 121 \\ 61 & .72 & 124 \\ 51 & 16.3 & 111 \\ 69 & 5.06 & 140 \\ 59 & 2.88 & 130 \\ 61 & 2.54 & 132 \\ 68 & 2.18 & 128 \\ 66 & 1.38 & 152 \\ 70 & 1.00 & 137 \\ 65 & 3.16 & 145 \\ 69 & 1.78 & 141 \\ 66 & 3.68 & 154 \\ 59 & 1.10 & 132 \\ 35 & 27.3 & 105 \\ 65 & 4.30 & 151 \\ 62 & 1.30 & 119 \\ 68 & 7.62 & 154 \\ 59 & 1.28 & 140 \\ 54 & 2.24 & 149 \\ 49 & 30.9 & 127 \\ 61 & 3.56 & 120 \\ 60 & 3.30 & 141 \\ 47 & 1.38 & 143 \\ 52 & 33.3 & 123 \\ 30 & 2.00 & 116\end{array}$

Phosphate, total as $\mathrm{PO}$

1 Pond $\mathrm{Cr}$ at Pendleton Road

$2 \mathrm{Mill} \mathrm{Cr}$ at Orell Road

3 Pond $\mathrm{Cr}$ at Manslick Road

5 SF Beargrass $\mathrm{Cr}$ at Winter Avenue

$6 \mathrm{SF}$ Beargrass $\mathrm{Cr}$ at Trevilian Way

$7 \mathrm{MF}$ Beargrass $\mathrm{Cr}$ at Old Cannons Lane

8 MF Beargrass $\mathrm{Cr}$ at Beals Branch Road

9 Spring Ditch at Private Drive

10 Muddy Fork at Mockingbird Valley Road

11 Goose $\mathrm{Cr}$ at U.S. Highway 42

12 Little Goose $\mathrm{Cr}$ at U.S. Highway 42

13 Goose $\mathrm{Cr}$ at old Westport Road

14 Pope Lick at Pope Lick Road

15 Floyds Fork at former State Highway 155

16 Chenoweth Run at Gelhaus Road

17 Fern $\mathrm{Cr}$ at Old Bardstown Road

18 Northern Ditch at Preston Highway

$19 \mathrm{Fishpool} \mathrm{Cr}$ at Bost Road

20 Southern Ditch at Minors Lane

21 Floyds Fork at Bardstown Road

22 Cedar $\mathrm{Cr}$ at Thixton Road

23 Pennsylvania Run at Mt. Washington Road

$24 \mathrm{Mi} 11 \mathrm{Cr}$ Cutoff at Dover Road

25 Harrods $\mathrm{Cr}$ at Hunting $\mathrm{Cr}$ Drive

26 Long Run at State Highway 1531

$\begin{array}{lcr}63 & 59.4 & 47.7 \\ 59 & 2.30 & 78.4 \\ 52 & 57.2 & 50.1 \\ 68 & 4.14 & 86.7 \\ 57 & 1.84 & 79.2 \\ 56 & 3.06 & 104 \\ 64 & 1.86 & 72.7 \\ 63 & 3.28 & 74.7 \\ 69 & 7.14 & 52.0 \\ 65 & 16.6 & 47.1 \\ 70 & 10.3 & 55.5 \\ 65 & 12.9 & 70.0 \\ 58 & 3.82 & 59.1 \\ 35 & 22.1 & 77.3 \\ 62 & 20.9 & 44.4 \\ 61 & 9.28 & 70.8 \\ 67 & 30.1 & 66.7 \\ 57 & 6.78 & 68.5 \\ 53 & 9.02 & 57.8 \\ 49 & 47.0 & 55.6 \\ 60 & 15.0 & 62.4 \\ 59 & 10.2 & 68.2 \\ 46 & 7.06 & 109 \\ 51 & 29.7 & 76.4 \\ 30 & 2.84 & 83.2\end{array}$

$\begin{array}{rr}3.7 & 22.4 \\ 7.6 & 9.4 \\ 2.6 & 44.0 \\ .4 & 4.8 \\ 8.2 & 9.8 \\ .8 & 2.9 \\ .6 & 1.6 \\ .1 & .7 \\ 2.5 & 4.5 \\ .1 & .3 \\ .3 & 1.5 \\ 2.4 & 1.9 \\ 3.2 & 16.2 \\ .4 & .6 \\ .3 & 2.3 \\ .7 & 6.9 \\ 9.4 & 30.0 \\ 5.9 & 10.4 \\ 1.7 & 9.8 \\ 7.0 & 64.7\end{array}$

$\begin{array}{rr}3.7 & 23.0 \\ 7.6 & 28.6 \\ 2.6 & 15.7 \\ .4 & 3.1 \\ 8.2 & 24.7 \\ .8 & 4.0 \\ .6 & 2.4 \\ .1 & 1.1 \\ 2.5 & 7.4 \\ .1 & .3 \\ .3 & .9 \\ .6 & 2.3 \\ 2.4 & 12.6 \\ 3.3 & 16.9 \\ 3.8 & 21.0 \\ 3.2 & 5.6 \\ .4 & 2.1 \\ .3 & 2.4 \\ .7 & 5.8 \\ 9.4 & 33.3 \\ 5.9 & 32.0 \\ 1.7 & 20.5 \\ 7.0 & 49.2 \\ 1.3 & 9.6 \\ 2.7 & 18.1 \\ & \end{array}$

23.0

15.7

3.1

24.7
4.0

2. 4 
Table 15. Estimates of mean annual loads of constituents in base flow in urban watersheds of Jefferson County, Kentucky, 1988-92--Continued

[Cr, Creek; SF, South Fork; MF, Middle Fork; ${ }^{\circ} \mathrm{C}$, degrees Celsius ]

\begin{tabular}{|c|c|c|c|c|c|}
\hline Site number and name & $\begin{array}{c}\text { Number } \\
\text { of } \\
\text { obser- } \\
\text { vations }\end{array}$ & $\begin{array}{l}\text { Mean } \\
\text { annual } \\
\text { load, } \\
\text { in tons }\end{array}$ & $\begin{array}{l}\text { Standard } \\
\text { error of } \\
\text { regression }\end{array}$ & $\begin{array}{l}\text { Flow duration } \\
\text { of greatest } \\
\text { sampled } \\
\text { discharge, } \\
\text { in percent }\end{array}$ & $\begin{array}{l}\text { Percentage of } \\
\text { load estimated } \\
\text { beyond range } \\
\text { of sampled } \\
\text { discharge }\end{array}$ \\
\hline
\end{tabular}

1 Pond $\mathrm{Cr}$ at Pendleton Road

$2 \mathrm{Mill} \mathrm{Cr}$ at Orell Road

3 Pond $\mathrm{Cr}$ at Manslick Road

5 SF Beargrass $\mathrm{Cr}$ at Winter Avenue

6 SF Beargrass $\mathrm{Cr}$ at Trevilian Way

7 MF Beargrass $\mathrm{Cr}$ at Old Cannons Lane

8 MF Beargrass $\mathrm{Cr}$ at Beals Branch Road

9 Spring Ditch at Private Drive

10 Muddy Fork at Mockingbird Valley Road

11 Goose $\mathrm{Cr}$ at U.S. Highway 42

12 Little Goose $\mathrm{Cr}$ at U.S. Highway 42

13 Goose $\mathrm{Cr}$ at old Westport Road

14 Pope Lick at Pope Lick Road

15 Floyds Fork at former State Highway 155

16 Chenoweth Run at Gelhaus Road

17 Fern $\mathrm{Cr}$ at Old Bardstown Road

18 Northern Ditch at Preston Highway

$19 \mathrm{Fishpool} \mathrm{Cr}$ at Bost Road

20 Southern Ditch at Minors Lane

21 Floyds Fork at Bardstown Road

22 Cedar $\mathrm{Cr}$ at Thixton Road

23 Pennsylvania Run at Mt. Washington Road

$24 \mathrm{Mi} 11 \mathrm{Cr}$ Cutoff at Dover Road

25 Harrods $\mathrm{Cr}$ at Hunting $\mathrm{Cr}$ Drive

26 Long Run at State Highway 1531

\section{Phosphorus, total as $\mathrm{P}$}

$\begin{array}{rrrrr}61 & 22.0 & 52.0 & 3.7 & 9.4 \\ 60 & 1.24 & 85.2 & 7.6 & 60.6 \\ 49 & 21.7 & 50.9 & 2.6 & 7.9 \\ 68 & 2.34 & 84.6 & .4 & 5.1 \\ 56 & .80 & 67.7 & 8.2 & 28.9 \\ 59 & 1.36 & 89.6 & .8 & 5.3 \\ 65 & 1.34 & 85.4 & .6 & 3.1 \\ 61 & 1.58 & 62.5 & .1 & .5 \\ 68 & 3.20 & 64.7 & 2.5 & 4.2 \\ 63 & 6.70 & 51.8 & .1 & .1 \\ 68 & 4.98 & 75.9 & .3 & .6 \\ 65 & 5.52 & 68.1 & .6 & 1.0 \\ 56 & 1.82 & 68.1 & 2.4 & 3.9 \\ 32 & 11.1 & 68.2 & 3.3 & 18.5 \\ 63 & 9.06 & 64.4 & 3.8 & 6.3 \\ 60 & 4.78 & 70.4 & 3.2 & 1.1 \\ 65 & 14.4 & 69.1 & .4 & 1.8 \\ 55 & 3.02 & 66.7 & .3 & 3.4 \\ 52 & 3.80 & 63.7 & .7 & 21.0 \\ 46 & 19.6 & 57.5 & 9.4 & 17.7 \\ 58 & 6.56 & 60.8 & 5.9 & 12.2 \\ 58 & 4.82 & 76.0 & 1.7 & 57.9 \\ 47 & 2.58 & 92.6 & 7.0 & 3.3 \\ 51 & 13.2 & 71.6 & 1.3 & 28.0 \\ 30 & 1.22 & 86.6 & 2.7 & \end{array}$

Phosphorus, orthophosphate, total as $\mathrm{P}$

1 Pond $\mathrm{Cr}$ at Pendleton Road

$2 \mathrm{Mill} \mathrm{Cr}$ at Orell Road

3 Pond $\mathrm{Cr}$ at Manslick Road

5 SF Beargrass $\mathrm{Cr}$ at Winter Avenue

$6 \mathrm{SF}$ Beargrass $\mathrm{Cr}$ at Trevilian Way

7 MF Beargrass $\mathrm{Cr}$ at Old Cannons Lane

8 MF Beargrass $\mathrm{Cr}$ at Beals Branch Road

9 Spring Ditch at Private Drive

10 Muddy Fork at Mockingbird Valley Road

11 Goose $\mathrm{Cr}$ at U.S. Highway 42

12 Little Goose $\mathrm{Cr}$ at U.S. Highway 42

13 Goose $\mathrm{Cr}$ at old Westport Road

14 Pope Lick at Pope Lick Road

15 Floyds Fork at former State Highway 155

16 Chenoweth Run at Gelhaus Road

17 Ferm $\mathrm{Cr}$ at Old Bardstown Road

18 Northern Ditch at Preston Highway

$19 \mathrm{Fishpool} \mathrm{Cr}$ at Bost Road

20 Southern Ditch at Minors Lane

21 Floyds Fork at Bardstown Road

22 Cedar $\mathrm{Cr}$ at Thixton Road

23 Pennsylvania Run at Mt. Washington Road

$24 \mathrm{Mill}$ Cr Cutoff at Dover Road

25 Harrods $\mathrm{Cr}$ at Hunting $\mathrm{Cr}_{r}$ Drive

26 Long Run at State Highway 1531

1 Pond $\mathrm{Cr}$ at Pendleton Road

$2 \mathrm{Mill} \mathrm{Cr}$ at Orell Road

3 Pond $\mathrm{Cr}$ at Manslick Road

5 SF Beargrass $\mathrm{Cr}$ at Winter Avenue

6 SF Beargrass $\mathrm{Cr}$ at Trevilian Way

9 Spring Ditch at Private Drive

10 Muddy Fork at Mockingbird Valley Road

11 Goose $\mathrm{Cr}$ at U.S. Highway 42

12 Little Goose $\mathrm{Cr}$ at U.S. Highway 42

13 Goose $\mathrm{Cr}$ at old Westport Road

14 Pope Lick at Pope Lick Road

15 Floyds Fork at former State Highway 155

16 Chenoweth Run at Gelhaus Road

17 Fern $\mathrm{Cr}$ at Old Bardstown Road

18 Northern Ditch at Preston Highway

21 Floyds Fork at Bardstown Road

22 Cedar $\mathrm{Cr}$ at Thixton Road

23 Pennsy lvania Run at Mt. Washington Road

25 Harrods $\mathrm{Cr}$ at Hunting $\mathrm{Cr}$ Drive

63
60
52
68
58
60
67
65
69
65
70
65
58
35
62
61
67
58
54
49
60
59
46
51
30

19.4

18.6

18.6
1.36

1.36
.58

.88

.88
1.04

1.04
2.34

5.40

3. 34

4. 22

1. 24

7.22

6.82

3. 02

9.84

2. 10

2.66

15.3

4. 90

3. 32

2. 30

9.68

.92

Barium, total as Ba

$15 \quad 1.50$

15

13

14
12

12
13

12

14

13
14

14
15

10

10
16

12
12

10

12
13

10
1.50
.12

1.34

1.34
.56

.48
.08

.18

.34

.24
.28

.06

1.46

.34
.14

.42

3.04

.28

.12
1.62
47.7

85.6

50.1

86.8

83.9

117

82.6

103

52.0

47.1

55.5

70.0

59.1

77.3

44.4

70.8

66.7

92.3

84.3

55.6

62.4

68.2

109

76.4

83.3

34.2

37.1

19.1

12.8

13.8

14.9

26.8

27.4

33.5

33.1

44.0

35.3

9.8

15.9

13.4

26.8

19.5

29.7
3.7

7.6
2.6

2.6
.4

8.2

.8

.6
.1
2.5

2.5
.1

.3

.6
2.4

3.3

3.8

3.2

.4
.3

.7
9.4

9.4
5.9

1.7

1.3
2.7

5.9

10.6
2.6

4.4

8.2

3.7

11.0

19.6

8.2

8.7

11.9

5.9

4.5

25.8

5.9

1.7
8.6
9.4

60.6
7.9

5. 1

5.3
12. 2

59.4

9.2

5.2

35.4
4.2

2.3

2.3
.3

2. 9

.1

1.0

4.2

14.3

8.5

6.7
.7

1.4

2.8

20.6

19. 1

14.7

61.0

2.8

35.1

25.8

54.0

21.8

20.2

27.7

13.4

38.7

46.8

1.3

19.9

29.4

35.9

32.7

17.6

2.2

64.5

35.3

35.3
12.6

11.4 
Table 15. Estimates of mean annual loads of constituents in base flow in urban watersheds of Jefferson County, Kentucky, 1988-92--Continued

[Cr, Creek; SF, South Fork; MF, Middle Fork; ${ }^{\circ} \mathrm{C}$, degrees Celsius ]

\begin{tabular}{|c|c|c|c|c|c|}
\hline Site number and name & $\begin{array}{c}\text { Number } \\
\text { of } \\
\text { obser- } \\
\text { vations }\end{array}$ & $\begin{array}{l}\text { Mean } \\
\text { annual } \\
\text { load, } \\
\text { in tons }\end{array}$ & $\begin{array}{l}\text { Standard } \\
\text { error of } \\
\text { regression }\end{array}$ & $\begin{array}{l}\text { Flow duration } \\
\text { of greatest } \\
\text { sampled } \\
\text { discharge, } \\
\text { in percent }\end{array}$ & $\begin{array}{l}\text { Percentage of } \\
\text { load estimated } \\
\text { beyond range } \\
\text { of sampled } \\
\text { discharge }\end{array}$ \\
\hline
\end{tabular}

1 Pond $\mathrm{Cr}$ at Pendlet on Road

3 Pond $\mathrm{Cr}$ at Manslick Road

9 Spring Ditch at Private Drive

\section{Chromium, total as $\mathrm{Cr}$}

$\begin{array}{rrrrr}15 & 0.80 & 128 & 5.9 & 14.8 \\ 13 & .66 & 131 & 2.6 & 2.2 \\ 13 & .02 & 79.0 & 3.7 & 18.8\end{array}$

\section{Copper total recoverable as $\mathrm{Cu}$}

1 Pond $\mathrm{Cr}$ at Pendleton Road

3 Pond $\mathrm{Cr}$ at Manslick Road

5 SF Beargrass $\mathrm{Cr}$ at Winter Avenue

9 Spring Ditch at Private Drive

11 Goose $\mathrm{Cr}$ at U.S. Highway 42

13 Goose $\mathrm{Cr}$ at old Westport Road

14 Pope Lick at Pope Lick Road

15 Floyds Fork at former State Highway 155

17 Fern $\mathrm{Cr}$ at Old Bardstown Road

18 Northern Ditch at Preston Highway

22 Cedar $\mathrm{Cr}$ at Thixton Road

23 Pennsylvania Rum at Mt. Washington Road

$\begin{array}{lcc}15 & .40 & 96.5 \\ 13 & .64 & 94.9 \\ 14 & .14 & 100 \\ 13 & .02 & 79.8 \\ 14 & .14 & 116 \\ 14 & .06 & 80.7 \\ 15 & .02 & 61.9 \\ 10 & .66 & 91.5 \\ 12 & .04 & 94.4 \\ 12 & .14 & 42.0 \\ 12 & .04 & 79.6 \\ 13 & .04 & 94.3\end{array}$

$\begin{array}{rr}5.9 & 12.7 \\ 2.6 & 26.0 \\ 4.4 & 24.4 \\ 3.7 & 9.8 \\ 19.6 & 44.1 \\ 8.2 & 8.5 \\ 8.7 & 27.0 \\ 11.9 & 32.4 \\ 4.5 & 11.5 \\ .4 & .7 \\ 5.9 & 13.7 \\ 1.7 & 9.8\end{array}$

$2 \mathrm{Mi} 11 \mathrm{Cr}$ at Ore11 Road

3 Pond $\mathrm{Cr}$ at Manslick Road

5 SF Beargrass $\mathrm{Cr}$ at Winter Avenue

6 SF Beargrass $\mathrm{Cr}$ at Trevilian Way

9 Spring Ditch at Private Drive

10 Muddy Fork at Mockingbird Valley Road

11 Goose $\mathrm{Cr}$ at U.S. Highway 42

12 Little Goose $\mathrm{Cr}$ at U.S. Hi ghway 42

13 Goose $\mathrm{Cr}$ at old Westport Road

15 Floyds Fork at former State Highway 155

16 Chenoweth Run at Gelhaus Road

17 Fern $\mathrm{Cr}$ at Old Bardstown Road

18 Northern Ditch at Preston Highway

21 Floyds Fork at Bardstown Road

22 Cedar $\mathrm{Cr}$ at Thixton Road

23 Pennsylvania Rum at Mt. Washington Road

25 Harrods $\mathrm{Cr}$ at Hunting $\mathrm{Cr}$ Drive

Iron, total as Fe

$\begin{array}{ccc}14 & 8.82 & 116 \\ 13 & 53.3 & 81.6 \\ 14 & 7.80 & 72.3 \\ 12 & 3.58 & 156 \\ 13 & 1.32 & 129 \\ 12 & 2.36 & 106 \\ 14 & 3.20 & 178 \\ 13 & 3.08 & 78.2 \\ 14 & 4.24 & 182 \\ 10 & 164 & 222 \\ 16 & 3.20 & 149 \\ 12 & 1.38 & 171 \\ 12 & 2.88 & 53.7 \\ 10 & 26.4 & 155 \\ 12 & 1.58 & 154 \\ 13 & 2.32 & 184 \\ 10 & 64.7 & 188\end{array}$

$\begin{array}{rr}10.6 & 60.5 \\ 2.6 & 26.3 \\ 4.4 & 8.5 \\ 8.2 & 9.0 \\ 3.7 & 13.6 \\ 11.0 & 37.9 \\ 19.6 & 49.3 \\ .3 & 1.1 \\ 8.2 & 19.9 \\ 11.9 & 37.6 \\ 5.9 & 6.0 \\ 4.5 & 2.7 \\ .4 & 2.9 \\ 25.8 & 20.3 \\ 5.9 & 15.2 \\ 1.7 & 4.4 \\ 8.6 & 12.7\end{array}$

Mercury, total recoverable as $\mathrm{Hg}$

6 SF Beargrass $\mathrm{Cr}$ at Trevilian Way 22 Cedar $\mathrm{Cr}$ at Thixton Road

23 Pennsylvania Run at Mt. Washington Road
12

12
12
13
$<.01 \quad 130$
$<.01 \quad 69.7$

$<.01 \quad 90.8$
8.2

5. 9

3.7
.02

Zinc, total as $\mathrm{Zn}$

1 Pond $\mathrm{Cr}$ at Pendleton Road

$2 \mathrm{Mill} \mathrm{Cr}$ at Orell Road

3 Pond $\mathrm{Cr}$ at Manslick Road

5 SF Beargrass $\mathrm{Cr}$ at Winter Avenue

6 SF Beargrass $\mathrm{Cr}$ at Trevilian Way

9 Spring Ditch at Private Drive

10 Muddy Fork at Mockingbird Valley Road

11 Goose $\mathrm{Cr}$ at U.S. Highway 42

12 Little Goose $\mathrm{Cr}$ at U.S. Highway 42

13 Goose $\mathrm{Cr}$ at old Westport Road

14 Pope Lick at Pope Lick Road

is Floyds Fork at former State Highway 155

16 Chenoweth Run at Gelhaus Road

$17 \mathrm{Fern} \mathrm{Cr}$ at Old Bardstown Road

18 Northern Ditch at Preston Highway

21 Floyds Fork at Bardstown Road

22 Cedar $\mathrm{Cr}$ at Thixton Road

23 Pennsylvania Run at Mt. Washington Road

25 Harrods $\mathrm{Cr}$ at Hunting $\mathrm{Cr}$ Drive

13 Goose $\mathrm{Cr}$ at old Westport Road

16 Chenoweth Run at Gelhaus Road

1 Pond $\mathrm{Cr}$ at Pendleton Road

$2 \mathrm{Mill} \mathrm{Cr}$ at Orell Road

6 SF Beargrass $\mathrm{Cr}$ at Trevilian Way

$\begin{array}{rc}4.02 & 142 \\ .08 & 131 \\ 1.32 & 154 \\ .50 & 97.2 \\ .90 & 132 \\ .20 & 98.1 \\ .88 & 128 \\ .94 & 146 \\ .14 & 87.3 \\ .38 & 149 \\ .16 & 91.3 \\ 2.42 & 89.3 \\ .40 & 105 \\ .10 & 124 \\ .66 & 113 \\ 4.06 & 111 \\ .32 & 103 \\ .20 & 127 \\ 5.10 & 132\end{array}$

$\begin{array}{lllll}13 & .06 & 79.4 & 8.2 & 22.5 \\ 15 & .06 & 67.1 & 5.9 & 14.9\end{array}$

2, 4-D, total

$15<.01$

$14<.01 \quad 179$
5.9

10.6

2.6

8.2

3.7

11.0

19.6

8.3

8.2
8.7

11.9

5. 9

4.5

.4
25.8

5.9

1. 7

8. 6

8. 2

5.9

22.5

2. 3

43.2

3.4

13.9

3.6

15.7

70.3

46. 4

6.9

31.5

28. 2

25. 1

3.8

1.5

65.7
28.2

6.1

16. 1

5.9

10.6

8.2

5. 6

24.2

24.6 